PNNL-19694

(1.) U.S. DEPARTMENT OF

Prepared for the U.S. Department of Energy

under Contract DE-AC05-76RL01830

\title{
Commercial Lighting Solutions Webtool Peer Review Report, Office Solutions
}

\author{
TA Beeson \\ CC Jones
}

February 2010

Pacific Northwest

NATIONAL LABORATORY 



\title{
DISCLAIMER
}

This report was prepared as an account of work sponsored by an agency of the United States Government. Neither the United States Government nor any agency thereof, nor Battelle Memorial Institute, nor any of their employees, makes any warranty, express or implied, or assumes any legal liability or responsibility for the accuracy, completeness, or usefulness of any information, apparatus, product, or process disclosed, or represents that its use would not infringe privately owned rights. Reference herein to any specific commercial product, process, or service by trade name, trademark, manufacturer, or otherwise does not necessarily constitute or imply its endorsement, recommendation, or favoring by the United States Government or any agency thereof, or Battelle Memorial Institute. The views and opinions of authors expressed herein do not necessarily state or reflect those of the United States Government or any agency thereof.

\author{
PACIFIC NORTHWEST NATIONAL LABORATORY \\ operated by \\ BATTELLE \\ for the \\ UNITED STATES DEPARTMENT OF ENERGY \\ under Contract DE-AC05-76RL01830
}

Printed in the United States of America

$$
\begin{aligned}
& \text { Available to DOE and DOE contractors from the } \\
& \text { Office of Scientific and Technical Information, } \\
& \text { P.O. Box 62, Oak Ridge, TN 37831-0062; } \\
& \text { ph: (865) 576-8401 } \\
& \text { fax: (865) 576-5728 } \\
& \text { email: reports@adonis.osti.gov }
\end{aligned}
$$

\author{
Available to the public from the National Technical Information Service, \\ U.S. Department of Commerce, 5285 Port Royal Rd., Springfield, VA 22161 \\ ph: (800) 553-6847$$
\text { fax: }(703) 605-6900
$$ \\ email: orders@ntis.fedworld.gov \\ online ordering: http://www.ntis.gov/ordering.htm
}




\title{
Commercial Lighting Solutions Webtool Peer Review Report Office Solutions
}

\author{
Tracy Beeson \\ Carol Jones
}

February 2010

Prepared for the

U.S. Department of Energy

Office of Energy Efficiency and Renewable Energy

Building Technologies Program

Under Contract DE-AC05-76RLO 1830

Pacific Northwest National Laboratory

Richland, Washington 99352 


\section{Executive Summary}

The Commercial Lighting Solutions (CLS) project directly supports the U.S. Department of Energy's Commercial Building Energy Alliance efforts to aid in the design of high-performance buildings. DOE has launched three CBEAs: the Retailer Energy Alliance (REA), the Commercial Real Estate Energy Alliance (CREEA), and the Hospital Energy Alliance (HEA). The alliances link commercial building owners, managers, and operators, by sector. A primary focus of the alliances is to reduce the energy consumption, greenhouse gas emissions, and operating expenses of member's buildings with the aid of advanced technologies, analytical tools, and capabilities emerging from DOE and the national laboratories.

DOE has initiated research to develop a series of flexible Commercial Technology Solutions to identify the low-energy technical pathways of attaining specific energy and economic goals for specific projects. The output is a series of web-based decision tools targeting commercial building design teams. Commercial Lighting Solutions (CLS) is one of these tools, and provides options that can be used in existing buildings as well as new construction and major renovation.

CLS creates energy-efficient best-practice lighting designs for widespread use. The lighting solutions are available to users via an interactive webtool that both educates and guides the user through the application of the solutions as well as providing immediate and project-specific feedback on the energy consequences of the various options.

In May 2009 version 1.0 of the webtool was released and included designs for box retail. This report, one of two FY10 critical commercial planning system milestones, summarizes the peer review of the beta version of the office solutions for the CLS webtool. Peer review input will be utilized in making improvements to the webtool in preparation for the release of version 2.0, which will include both office and retail lighting solutions.

In order to collect the level of detail desired from the peer review process, input was requested from professionals across the range of fields that would use and benefit from the tool. PNNL developed a list of potential reviewers and proactively invited both written input and input from qualitative interviews. Reviewers included (6) owner representatives from the CBEA, (4) lighting designers, (3) lighting manufacturers, (1) architects, (1) engineers, (2) state energyefficiency program managers and (2) utility program managers. Two internal PNNL staff also reviewed the tool for this report. (see Appendix A). The diverse representation of members ensured that all aspects of the tool were analyzed from many perspectives.

The methodology for the peer review process included gathering input from interested stakeholders, analysis of the comments, and organization of the comments into categories for prioritization against a set of criteria. Based on this process, PNNL developed recommendations about which feedback will be addressed for the release of version 2.0 of the webtool at the LIGHTFAIR ${ }^{\circledR}$ International tradeshow and conference in Las Vegas, Nevada in May 2010.

Due to the volume of data ( $\sim 500$ individual comments) the methodology for addressing the peer review comments was central to the success of the ultimate goal of improving the tool. All of the comments were imported into a master spreadsheet, and then grouped and organized into several

categories. Solutions to each comment were then rated by importance and feasibility to determine both the short and long term practicality of the recommendations. The rating system was used as an analytical tool, but the results were interpreted thoughtfully to ensure that the rating was not the sole factor in determining which comments were recommended for near-term resolution. 
The report provides a list of the top ten most significant and relevant improvements that will be incorporated into the webtool for version 2.0 as well as appendices containing additional detail on all short-term priorities. Peer review comments that are considered high priority by the reviewers and the CLS team but cannot be completed for the version 2.0 release in May at Lightfair are listed as long-term recommendations.

The list of the top ten most significant and relevant improvements that PNNL will implement by Lightfair 2010 are listed below. These tasks may involve numerous smaller steps for completion, but they are provided here in a more general summary format for clarity.

(1) Increase clarity throughout the tool using established conventions (i.e., definitions of terms in glossary and /or mouse-overs). (Ticket 1)

(2) Add additional building codes to the drop-down code menu (e.g., Title 24 and IECC 2009) (Ticket 7)

(3) More clearly express to users the process for navigating through the vignette and controls selections. (Ticket 9)

(4) Provide user inputs and energy savings estimates from the Energy Estimator tool in a format (Excel table) usable by utilities. (Ticket 10)

(5) Provide a drop-down menu that provides guidance on visible transmittance values associated with different window types. (Ticket 18)

(6) Represent controls guidance in the summary and implementation PDFs in a way that shows controls are an integral part of the lighting solution and not an afterthought and clean up formatting and presentation of all download material. (Tickets 27-29)

(7) Ensure that users consciously chose a baseline code for their project. (Ticket 43)

(8) Clarify confusion associated with user input of area by adding a way to input square footage for typical rooms. (Ticket 46)

(9) Modify the project description page to be "building-neutral" to prevent confusion. (Ticket 49)

(10) Expand luminaire lists to adjust for the necessary ballast depending on the control strategies applied. (Ticket 22) 


\section{Contents}

EXECUTIVE SUMMARY iii

1.0 BACKGROUND 1

1.1 Commercial Lighting Solutions and the Commercial Buildings Energy Alliances 1

1.2 Commercial Lighting Solutions Webtool 2

1.3 Peer Review Task Description 2

1.4 Objectives 2

2.0 METHODOLOGY 2

2.1 Peer Review Data collection 3

2.1.1. Reviewers 3

2.1.2. Webtool Content 3

2.1.3. Peer Review Input Format 3

2.1.4. Process 4

2.2 Analysis and Prioritization 4

2.2.1. Spreadsheet Summary 4

2.2.2. Comments Assigned to Actions/Solutions 5

2.2.3. Importance and Feasibility Ratings 5

2.2.4. Overall Rankings, Short-term and Long-term Goals 6

2.2.5. Unrated Comments 7

3.0 RECOMMENDATIONS $\quad 7$

3.1 Development of Recommendations 7

3.1.1. Prioritization Ranges $\quad 8$

3.2 Top Ten List of Priority Short-Term Recommendations $\quad 8$

3.3 Action Tickets 9

3.4 Peer Review Input Data 9

3.5 List of Long-Term Priority Recommendations 9

4.0 CONCLUSION 10

APPENDIX A: REVIEWER LIST A-1

APPENDIX B: WEBTOOL PAGE REFERENCE FILE B-1

APPENDIX C: PEER REVIEW INPUT SPREADSHEET C-1

APPENDIX D: TICKET LIST

APPENDIX E: MASTER COMMENTS LIST $\quad$ E-1 


\section{Tables}

Table 1. Importance Ratings

Table 2. Feasibility Ratings. 


\subsection{Background}

\subsection{Commercial Lighting Solutions and the Commercial Buildings Energy Alliances}

Commercial buildings currently account for 19 percent of total U.S. energy consumption and carbon dioxide emissions, and are projected by the Energy Information Administration to experience significant growth over the next two decades. Lighting accounts for fully $25 \%$ of commercial buildings energy use. To increase the energy efficiency of this sector, in both new construction and the large stock of existing buildings, the U.S. Department of Energy (DOE) has launched three CBEAs: the Retailer Energy Alliance (REA), the Commercial Real Estate Energy Alliance (CREEA), and the Hospital Energy Alliance (HEA). The alliances link commercial building owners, managers, and operators, by sector, who want to reduce the energy consumption, greenhouse gas emissions, and operating expenses of their buildings with the advanced technologies, analytical tools, and capabilities emerging from DOE and the national laboratories. These informal associations function in a number of capacities, including:

- Conducting demonstration, dissemination, and technical assistance activities to encourage and assist adoption of technologies, practices, and policies;

- Sharing best practices, energy-use measurement, and benchmarking —in essence, serving as organic, real-time information networks;

- Tapping the technical expertise of DOE and its national laboratories to shape federal $R \& D$ to advance the business case for energy-efficient buildings;

- Deploying advanced technology through technology specifications and informationsharing within commercial subsectors;

- Developing training materials and courses for building professionals and trades to be deployed by alliance partners;

- Developing and disseminating public education materials on benefits and costeffectiveness of high-performance, energy-efficient buildings.

DOE has initiated research to develop a series of flexible Commercial Technology Solutions to identify the low-energy technical pathways of attaining specific energy and economic goals for specific projects. The output is a series of Web-based decision tools targeting commercial building design teams. Commercial Technology Solutions can be used to evaluate system-level packages of technologies, such as lighting (PNNL), daylighting (NREL), supermarket refrigeration (ORNL), and packaged HVAC (PNNL). These Web-based tools give commercial building designers and operators guidance on improving both their existing building systems' efficiency and that of new buildings."

The Commercial Lighting Solutions (CLS) project directly supports the Commercial Building Energy Alliance efforts to significantly improve the energy performance of existing buildings and new construction. . Lighting Solutions optimized for energy efficiency, cost effectiveness, and occupant satisfaction are critical to the end-product success of 50\% (and greater) energy savings against ASHRAE Std. 90.1-2004. Any endeavor to realize deep energy savings must include Lighting Solutions as part of its "solution set" because fully $1 / 4$ of commercial buildings energy 
use (on average) is lighting; sector wide, commercial buildings require 4.5 source quads per year to provide illumination services. ${ }^{1}$

Lighting Solutions have been developed for both retail and office buildings to deliver bestpractice guidance to the Alliances and the market at large. Critical to the success of the webtool is stakeholder input, which has been gathered via a peer review input process. This report summarizes the findings of the CLS Peer Review process for the office solutions.

\subsection{Commercial Lighting Solutions Webtool}

The webtool is the delivery mechanism for the Lighting Solutions. It is critical to widespread usage of the Lighting Solutions and includes a strong interactive element that both educates and guides the user through the application of the Lighting Solutions as well as providing immediate and project-specific feedback on the energy consequences of the various options.

\subsection{Peer Review Task Description}

The Peer Review task covers the process by which stakeholders provide input to the Lighting Solutions. The Peer Review process described in this report is limited to the beta version of the CLS webtool, which contains retail and office lighting solutions. Since retail buildings were thoroughly reviewed before the release (see Commercial Lighting Solutions, Webtool Peer Review Report, PNNL-18464) of version 1.0 in May 2009, the present review focuses primarily on the office solutions. The next draft of this report will augment these findings with comments on the Energy Estimator portion of the tool. As development continues on the webtool, the peer review will be phased and ongoing in support of new solutions, continuous improvement to existing solutions, and partner-contributed solutions.

\subsection{Objectives}

The objectives of the peer review were to:

(1) Gather high-quality feedback on the CLS webtool from a representative group of stakeholders.

(2) Analyze the peer review input data to categorize similar comments, determine possible solutions, and weigh and prioritize input against a set of decision criteria.

(3) Develop recommendations about tasks that should be undertaken to improve the webtool for the 2.0 release at Lightfair 2010 with the time and funds that are available.

(4) Develop recommendations about tasks that should be considered as longer-term goals for the continuous improvement of the webtool.

\subsection{Methodology}

The methodology for the peer review process included data collection (stakeholder input), analysis of the comments, and organization of the input into categories for prioritization of the comments against a set of criteria. Based on this process, recommendations were developed to prioritize what feedback should be addressed for the 2.0 release of the webtool.

\footnotetext{
${ }^{1} 2009$ Buildings Energy Data Book http://buildingsdatabook.eren.doe.gov/TableView.aspx?table=3.1.4
} 


\subsection{Peer Review Data collection}

The goal of data collection was to capture sufficient feedback from each of our target user groups, in an efficient and thorough process.

\subsubsection{Reviewers}

In order to collect the level of detail desired from the peer review process, input was requested from professionals across the range of fields that would use and benefit from the tool. PNNL developed a list of potential reviewers and proactively invited both written input and input from qualitative interviews. Reviewers included (6) owner representatives from the CBEA, (4) lighting designers, (3) lighting manufacturers, (1) architect, (1) engineer, (2) state energy-efficiency program managers and (2) utility program managers. Two internal PNNL staff also reviewed the tool for this report. (see Appendix A). The diverse representation of members ensured that all aspects of the tool were analyzed from many perspectives.

\subsubsection{Webtool Content}

The peer review included the following pages of the webtool: (1) sign-in, (2) new project, (3) project description, (4) key plan, (5) space type summary, (6) space description wizard, (7) vignette description (8) controls template options, (9) controls template description, (10) download materials . (see Appendix B).

\subsubsection{Peer Review Input Format}

In order to capture all of the feedback in an easily quantifiable manner, an Excel spreadsheet was designed to help the reviewers categorize their comments on the webtool (see Appendix C). The spreadsheet provided space for reviewers to include important information regarding their operating system and web browser so the web team could address functionality and compatibility issues once all of the input was received. Each comment was assigned a reference number to more easily track the progress of addressing the many comments received. There was also a column to record the space type and reference web page to assure that the context of the comment was sufficiently understood.

There were six categories in the spreadsheet that reviewers were asked to consider:

(1) appearance/aesthetics,

(2) ease of use/flow,

(3) functionality,

(4) content,

(5) download material, and

(6) General comments.

Providing guidance questions during the interview process encouraged a more comprehensive review and helped with the data analysis process.

As the comments were received they were compiled into a master spreadsheet for analysis, diagnosis, and resolution. 


\subsubsection{Process}

The project team scheduled meetings with interested parties to ensure that feedback was obtained in a manner similar to qualitative interviews. Most frequently an online meeting approach was used so the CLS team could observe the reviewer navigate through the tool and capture thoughts and questions in real time. Often through this dialogue the interviewer was able to more clearly identify the root cause of the problems, and identify potential solutions that would resolve concerns. The interviewer acted as the recorder for the reviewer's feedback, and then sent the spreadsheet input form to the reviewer after the interview so they could confirm the reviewer's recorded comments and add additional comments as they continued to spend time working with the tool. Some sessions were recorded with screen capture software to further illuminate the user experience for the reviewer and the software developers.

Reviewers felt that they could concentrate better on the review process because they did not have to go back and forth between the webtool and typing their thoughts into the comment sheet. Additionally, the PNNL interviewer was often able to catch issues and problems through the observation process that either would not have been noticed by the reviewer, or would not have been characterized clearly. The dialogue was also very useful in developing specific ideas about potential improvements to the webtool. After reviewing the peer review comments, the PNNL web development team said that the comments were significantly more useful and actionable than typical peer review input due to the live meeting interview approach.

The focus of the peer review was to identify problems and opportunities for improvement. Consequently, PNNL did not include the positive comments in formal evaluation process. Positive comments were unrated and are contained in a separate list within the data set in Appendix E. Reviewers almost universally liked the aesthetic appearance of the tool, the simplicity of the workflow, and the ability to see energy savings impacts as choices were made. The goal of the team will be to make improvements to the tool without losing the features that were widely appreciated by reviewers.

By the end of the peer review input period, 17 reviewers had been interviewed and 2 more provided detailed written input for a total of $\sim 500$ comments.

\subsection{Analysis and Prioritization}

Once all of the comments were imported into a master spreadsheet, they were grouped and organized into several categories. Strategies for each comment were then rated in several categories to determine the practicality of resolving the concerns of the commenter. After several additional filters to the data, short-term and long-term goals were established based on the ratings. The rating system was used as an analytical tool, but the results were viewed thoughtfully to ensure that they were not the sole factor in determining the practicality of comment resolution.

\subsubsection{Spreadsheet Summary}

Columns in the spreadsheet were expanded from the truncated peer review input form to support the data analysis, including the following: commenter ID number, action/solution, importance, feasibility, short-term rating, long-term rating, and status.

The comment type column retained the five general categories reviewers were asked to recognize on the peer review input form. Ratings on a scale of 1-5 were assigned to rank the frequency and priority of the comments that ultimately established the 'importance"rating. While the same 
rating scale was used for the ease of implementation, duration, and risk/complication, the importance and feasibility ratings were weighted together differently to attain the overall rating for the short and long term. This process is discussed in detail in section 2.2.4. The final two columns helped to further categorize and track the comments. The action/solution column linked to a list of more general issues that grouped comments with similar solutions. The status column is the working record of where the PNNL team stands in respect to resolving the issue.

Due to the diversity of the comments, separate tabs were created to support the analysis process including (1) content, (2) images, (3) user interface, (4) daylighting, (5) additions, (6) vignette text, (7) template text, (8) functionality, (9) controls templates, (10) PDFs, (11) luminaires, (12) phase 2, (13) retail, and (14) presentation order. This categorization simplified the process and allowed feasibility rankings to be assigned by the appropriate PNNL staff. This organization of comments also allowed for more specific action/solution categories to be assigned within the tabs.

\subsubsection{Comments Assigned to Actions/Solutions}

With the large quantity of comments received, it was important to divide them into refined categories that represented the type of action that would yield a solution. Comments were turned into proposed actions so that they could be rated in terms of importance and feasibility. When the team resolves a specific issue, then each comment that was categorized under the given action/solution can then be addressed individually to ensure completion.

This process allowed us to review similar comments in proximity to each other, and supported the process of turning comments into actionable solutions for future delegation and resolution by the team. The actions that were established in response to the peer review comments were developed as "tickets" and are shown in full in Appendix D. The tickets will either be entered into the CLS Sharepoint site for the technical team, or Rally (online development resource tool), for the respective groups to track progress and ticket resolution.

\subsubsection{Importance and Feasibility Ratings}

The overall importance of each category was determined by assigning a 1-5 rating for (1) the frequency of the comment and (2) the priority of the comment as it pertains to the complete webtool. The ratings were established using the guidelines as shown in the Table 1.

Table 1. Importance Ratings

\begin{tabular}{|l|l|}
\hline \multicolumn{2}{|c|}{ IMPORTANCE } \\
\hline \multicolumn{1}{|c|}{ Frequency } & \multicolumn{1}{c|}{ Importance of Comment } \\
\hline 1- only time comment came up & 1- pure opinion \\
2- comment repeated by someone & 2- opinion with foundation \\
3- comment has come up several & 3- would enhance tool \\
times & 4- tool loses effectiveness without implementation \\
4- comment came up frequently & 5- tool is incomplete without \\
5- almost everyone mentioned it & \\
\hline
\end{tabular}

The two subcategories were given a weighted average to attain the overall rating of importance. The frequency comments comprised $20 \%$ of the overall importance rating. The rating for the 
priority of the comment accounted for $80 \%$ of the overall importance. Although frequency is an important measure, it should not be weighted equally with importance because we had a very diverse set of reviewers. There may be a small number of comments from a particular constituency (e.g., owners, utilities) that is inherently more valuable due to the strategic importance of the stakeholder group.

The feasibility of implementing each concern was determined by rating the (1) ease of implementing the solution, (2) the uncertainty and/or risk of its implementation, and (3) the time duration of the implementation. After each comment was rated in the applicable subcategories, the priority of each subcategory was considered and weighted in importance for determining each comment's overall rating in terms of importance and feasibility. The guide used to establish the ratings is detailed below.

Table 2. Feasibility Ratings.

\begin{tabular}{|l|l|l|}
\hline \multicolumn{1}{|c|}{ FEASe of Implementation } & \multicolumn{1}{|c|}{ Risk/Uncertainty } & \multicolumn{1}{c|}{ Duration } \\
\hline $\begin{array}{l}\text { 1- redoing all previous efforts } \\
\text { 2- extensive alterations/ } \\
\text { additions }\end{array}$ & $\begin{array}{l}\text { 1- could increase liability } \\
\text { 2- complications might outweigh } \\
\text { benefits }\end{array}$ & 1-years \\
3- substantial amount of work & 3- will complicate things \\
4- slightly involved change & 4- might complicate things & 3- weeks \\
5- simple change & 5- practically no added risk & 4- days \\
\end{tabular}

The feasibility rating was established by weighting the importance of the subcategories. The ease of implementation and risk/uncertainty subcategories were each determined to represent $30 \%$ of the feasibility rating, and the duration category was weighted at $40 \%$.

Once the overall importance and feasibility ratings were determined the sorting tool was used to find the most important and feasible comments. The PNNL team came to a consensus that the final ratings accurately portrayed the importance and feasibility of the tasks that needed to be completed in response to the peer review input.

\subsubsection{Overall Rankings, Short-term and Long-term Goals}

The importance and feasibility ratings were then weighted and summed to attain an overall ranking ranging from $0-50 .{ }^{2}$ There were two overall rankings, one for short-term goals that can reasonably be addressed before the release of version 2.0 at LightFair 2010 and another for longterm goals that will greatly enhance the tool but cannot be addressed until after the release. .

The short-term goals were primarily determined by the feasibility of completing the task prior to the 2.0 release. The importance of the comment accounted for $40 \%$, while feasibility represented $60 \%$ of the short-term goal ranking. This weighting process still accounted for the importance of

${ }^{2}$ The overall ranking was multiplied by 10 so that the range could be viewed as whole numbers rather than decimals to better illustrate the variances.

February 2010 
the comments, but brought less detrimental stylistic and agreement errors with higher feasibility to the top of the list. Examples of comments that attained higher short-term rankings than longterm rankings are (1) including definitions to terms and acronyms; (2) omitting any discrepancies between the images and the recommendations; and (3) limiting the number of significant digits for luminaire data.

The long-term goal rankings were assigned a different weighting of the importance and feasibility ratings that put much more emphasis on the importance of the comments. In this case, feasibility accounted for $15 \%$ of the total, where importance was valued at $85 \%$. This process ensured that extremely important aspects of the webtool that are more difficult to implement such as (1) programmatically establishing communications with the COMcheck energy code compliance tool via data exports; (2) providing additional guidance for daylighting; or (3) incorporating a wizard for retail that will provide users with only spaces applicable to their building type were high on the list of priorities for the path forward post-Lightfair.

In consideration of timeline and resources, the overall ranking was reviewed to determine thresholds for practical resolution of the comments. All comments that were ranked above 40 in the short term had their actionable solutions made into tickets to be resolved. The practicality of resolving the comments that were between 35 and 39 was analyzed on an individual basis. All comments below the short-term ranking of 35 were either established as "LT" for long-term goals, or To Be Determined (TBD) for comments that did not have an obvious solution.

\subsubsection{Unrated Comments}

There were a number of comments that were either unrated, or have not been made into actionable ticket items yet. Some reasons for their exclusion include: Some ratings had a higher level of uncertainty because they needed additional research to determine the level of effort required to resolve the comment. Although the solutions to these comments might have a rating in the high $30 \mathrm{~s}$, the actual solution requires greater consideration than what could be established before the peer review report deadline. The status of these comments remains TBD.

- Comments that were made infrequently regarding the functionality of the webtool that we have been unable to replicate have a status TBD.

- Comments that expressed opinions, e.g., "perspectives are very helpful," or "tool is very intuitive and easy to use" were not rated at all, but included on a separate list within the data set in Appendix E. There are 20 comments that fall under this category.

The numbered ratings were designed to be a guide and were not the sole factor driving prioritization. The team analyzed the ratings and threshold for comments and adjusted the comment priority accordingly. The final prioritization allows for modification from the ranking, but allows flexibility in establishing the ultimate viability of each action item.

\subsection{Recommendations}

\subsection{Development of Recommendations}

The spreadsheet and rating system proved to be very helpful tools in establishing reasonable goals for the short and long-term enhancements of the webtool. The organization of the spreadsheet has 
provided a straightforward range of recommendations for the improvements to the CLS that are clearly categorized and applicable.

At the completion of the rating process, all of the comments were ranked based on the assumptions and weighting factors indicated above into a list ranging from $0-50$. These overall rankings provided guidance about which tasks can and should be the highest priorities for inclusion in the CLS webtool version 2.0, scheduled for release at Lightfair.

\subsubsection{Prioritization Ranges}

For the purposes of prioritization, the following ranges were identified for $\mathrm{A}, \mathrm{B}$ and $\mathrm{C}$ priorities:

- Comments ranked at 40 and above were considered highest (A) priority, and will be addressed for version 2.0 .

- Comments in the range from 35-39 were considered high priority (B), and likely but not definitely achievable for version 2.0. A concerted best effort will be made to resolve comments in this range.

- Comments in the range from 30-34 were considered medium priority (C), and will only be addressed for version 2.0 if there is time after priorities A and B are completed.

- Comments in the range from 0-29 will be considered for the next version of the software.

\subsection{Top Ten List of Priority Short-Term Recommendations}

The list of the top ten most significant and relevant improvements that PNNL will implement by Lightfair 2010 are listed below. These tasks may involve numerous smaller steps for completion, but they are provided here in a more general summary format for clarity.

(1) Increase clarity throughout the tool using established conventions (i.e., definitions of terms in glossary and /or mouse-overs). (Ticket 1)

(2) Add additional building codes to the drop-down code menu (e.g., Title 24 and IECC 2009) (Ticket 7)

(3) More clearly express to users the process for navigating through the vignette and controls selections. (Ticket 9)

(4) Provide user inputs and energy savings estimates from the Energy Estimator tool in a format (Excel table) usable by utilities. (Ticket 10)

(5) Provide a drop-down menu that provides guidance on visible transmittance values associated with different window types. (Ticket 18)

(6) Represent controls guidance in the summary and implementation PDFs in a way that shows controls are an integral part of the lighting solution and not an afterthought and clean up formatting and presentation of all download material. (Tickets 27-29)

(7) Ensure that users consciously chose a baseline code for their project. (Ticket 43)

(8) Clarify confusion associated with user input of area by adding a way to input square footage for typical rooms. (Ticket 46)

(9) Modify the project description page to be "building-neutral" to prevent confusion. (Ticket 49) 
(10) Expand luminaire lists to adjust for the necessary ballast depending on the control strategies applied. (Ticket 22)

\subsection{Action Tickets}

For the purposes of comprehensive reporting, the comments received (included as a "ticket list") have been included into a chart format and shown with A and B priorities in Appendix D.

\subsection{Peer Review Input Data}

The master spreadsheet containing all of the peer review input is contained in Appendix E.

\subsection{List of Long-Term Priority Recommendations}

As expected, there are a number of recommendations considered to be high priority by the reviewers and by PNNL but are too ambitious to be completed for version 2.0. Below is a list of tasks for consideration for the next version of the webtool.

\begin{tabular}{|l|l|}
\hline \multicolumn{1}{|c|}{ Long Term Goals } & Reason for the Long-Term Nature of the Goal \\
\hline $\begin{array}{l}\text { Incorporate a wizard for retail "box" buildings } \\
\text { that gathers building characteristics and } \\
\text { ensures that the vignettes offered are } \\
\text { appropriate. Users should know up-front what } \\
\text { the space parameters for the retail vignettes } \\
\text { are }\end{array}$ & $\begin{array}{l}\text { A wizard for retail buildings will require } \\
\text { extensive work to the UI and organization of the } \\
\text { retail vignette content. A thorough understanding } \\
\text { of the division of the various box retail stores } \\
\text { must be established prior to integrating the } \\
\text { content into the webtool. }\end{array}$ \\
\hline $\begin{array}{l}\text { Collaborate with COMcheck to reduce the } \\
\text { amount of work required by the user, thereby } \\
\text { increasing the desirability and possibility of } \\
\text { widespread adoption. }\end{array}$ & $\begin{array}{l}\text { CLS has intended to collaborate with the } \\
\text { COMcheck web service for some time now. } \\
\text { Recent enhancements to COMcheck have } \\
\text { introduced the possibility of data sharing. }\end{array}$ \\
\hline $\begin{array}{l}\text { Increase the diversity of the building types to } \\
\text { increase applicability to those utilities that } \\
\text { want to use CLS in their rebate and incentive } \\
\text { programs. }\end{array}$ & $\begin{array}{l}\text { Additional designs will require lighting designers } \\
\text { to be subcontracted, and integrating any new } \\
\text { vignettes or building types will be a substantial } \\
\text { amount of work. }\end{array}$ \\
\hline $\begin{array}{l}\text { Help standardize the webtool as a common } \\
\text { and accessible program through training and } \\
\text { outreach programs. }\end{array}$ & $\begin{array}{l}\text { Including the data collection interface is a } \\
\text { substantial software development effort and } \\
\text { needs to be done with the intention of connecting } \\
\text { to compliance tools. }\end{array}$ \\
\hline
\end{tabular}




\begin{tabular}{|l|l|}
\hline $\begin{array}{l}\text { Consider providing load profiles to further } \\
\text { assist utilities in developing load reduction } \\
\text { programs. }\end{array}$ & $\begin{array}{l}\text { As the benefit the webtool offers to utilities } \\
\text { becomes apparent, future enhancements such as } \\
\text { load profiling should be considered. Making this } \\
\text { inclusion would comprise of a large shift in the } \\
\text { application of data, algorithms, and database } \\
\text { structuring. }\end{array}$ \\
\hline $\begin{array}{l}\text { Provide additional guidance on Daylighting } \\
\text { envelope design for various climates. }\end{array}$ & $\begin{array}{l}\text { There is currently no party that provides this type } \\
\text { of comprehensive instruction for a specific } \\
\text { building type. Numerous research papers will } \\
\text { have to be applied by an expert in the field. }\end{array}$ \\
\hline
\end{tabular}

\subsection{Conclusion}

The peer review data collection process was effective in gathering sufficient input from the appropriate range of stakeholders in a way that allowed for a meaningful understanding of the issues and useful ideas about improvements. The data analysis and prioritization process was thorough and efficient, yielding reasonable goals and a clear path forward. The master spreadsheet will continue to be used throughout the resolution of comments, and will be an effective tool for future peer reviews. PNNL plans to use the peer review input, in both the near and long term, to improve the value of the CLS webtool. 


\section{Appendix A: Reviewer List}

\begin{tabular}{|c|c|c|c|}
\hline Category & First name & Last Name & Contact Information \\
\hline \multicolumn{4}{|c|}{ External Reviewers } \\
\hline \multicolumn{4}{|c|}{ Categories: Engineer, Lighting Designer, Manufacturer, Architect, Owner, State, Utility, } \\
\hline Engineer & Nick & Ferzacca & $\begin{array}{l}\text { Architectural Engineers, Inc. } \\
\text { Boston, MA } \\
\text { office } 617542 \text { 0810, x111 } \\
\text { nferzacca@arcengrs.com }\end{array}$ \\
\hline $\begin{array}{l}\text { Lighting } \\
\text { Designer }\end{array}$ & Kathy & Abernathy & $\begin{array}{l}\text { Abernathy Lighting Design, Inc. } \\
\text { N. Providence RI } \\
\text { office 401-233-4412 } \\
\text { kathy@abernathylightdesign.com }\end{array}$ \\
\hline $\begin{array}{l}\text { Lighting } \\
\text { Designer }\end{array}$ & Leslie & Davis & $\begin{array}{l}\text { Leslie Davis Lighting } \\
\text { Berkely, CA } \\
\text { cell 510-798-8635 } \\
\text { lesliedavisltg@comcast.net }\end{array}$ \\
\hline $\begin{array}{l}\text { Lighting } \\
\text { Designer }\end{array}$ & Wilson & Dau & $\begin{array}{l}\text { Dau Design and Consulting Inc. } \\
\text { Calgary, AB T2Z 3V8, Canada } \\
\text { office 403-714-9457 } \\
\text { wilson.dau@dau.ca }\end{array}$ \\
\hline $\begin{array}{l}\text { Lighting } \\
\text { Designer }\end{array}$ & Hayden & McKay & $\begin{array}{l}\text { HLB Lighting Design } \\
\text { New York, NY } \\
\text { office 212-674-5580 ext } 133 \\
\text { hmckay@HLBlighting.com }\end{array}$ \\
\hline Manufactuer & Craig & Dilouie & $\begin{array}{l}\text { Zing Communications } \\
\text { Calgary, AB T2T 0C8, Canada } \\
\text { office 403-802-1809 } \\
\text { cdilouie@zinginc.com }\end{array}$ \\
\hline Manufacturer & Jim & Yorgey & $\begin{array}{l}\text { Lutron Electronics, Inc. } \\
\text { Coopersburg, PA } \\
\text { office 610-282-3800 } \\
\text { jyorgey@lutron.com }\end{array}$ \\
\hline Manufacturer & Dorene & Maniccia & $\begin{array}{l}\text { Watt Stopper/Legrand } \\
\text { Birmingham, AL } \\
\text { office 205-271-3403 } \\
\text { dorene.maniccia@WattStopper.com }\end{array}$ \\
\hline
\end{tabular}




\begin{tabular}{|c|c|c|c|}
\hline Category & First name & Last Name & Contact Information \\
\hline Architect & Amy & Cortese & $\begin{array}{l}\text { New Buildings Institute } \\
\text { Vancouver, WA } \\
360-567-0950 \times 112 \\
\text { amy@newbuildings.org }\end{array}$ \\
\hline Owner & Russ & Parrish & $\begin{array}{l}\text { C.B. Richard Ellis } \\
\text { Tampa, FL } \\
\text { russ.parrish@cbre.com }\end{array}$ \\
\hline Owner & Eric & Smith & $\begin{array}{l}\text { C.B. Richard Ellis } \\
\text { Cincinnati, OH } \\
\text { eric.f.smith@cbre.com }\end{array}$ \\
\hline Owner & Dave & Pogue & $\begin{array}{l}\text { C.B. Richard Ellis } \\
\text { San Jose, CA } \\
\text { office 408-453-7444 } \\
\text { dave.pogue@cbre.com }\end{array}$ \\
\hline Owner & Chris & Magee & $\begin{array}{l}\text { MGM Mirage } \\
\text { Las Vegas, NV } \\
\text { office 702.590.5058 } \\
\text { cmagee@mgmmirage.com }\end{array}$ \\
\hline Owner & David & Robinson & $\begin{array}{l}\text { Hines / Conceptual Construction } \\
\text { Houston, TX } \\
\text { office 713-966-7658 } \\
\text { david.robinson@hines.com }\end{array}$ \\
\hline Owner & Jack & Beutell & $\begin{array}{l}\text { Hines } \\
\text { Houston, TX } \\
\text { office 713-966-7777 } \\
\text { Jack.Beuttell@hines.com }\end{array}$ \\
\hline State & Marilyn & Dare & $\begin{array}{l}\text { NYSERDA } \\
\text { Albany, NY } \\
\text { office 518-862-1090, ext. } 3348 \\
\text { mjd@nyserda.org }\end{array}$ \\
\hline State & Jennifer & Manierre & $\begin{array}{l}\text { NYSERDA } \\
\text { Albany, NY } \\
\text { office 518-862-1090 x3406 } \\
\text { jtm@nyserda.org }\end{array}$ \\
\hline Utility & Kelly & Cota & $\begin{array}{l}\text { National Grid } \\
\text { Waltham, MA } \\
\text { office 781-907-1554 } \\
\text { kelly.cota@us.ngrid.com }\end{array}$ \\
\hline
\end{tabular}




\begin{tabular}{|l|l|l|l|}
\hline Category & First name & Last Name & Contact Information \\
\hline Utility & Paul & Lentine & $\begin{array}{l}\text { Northeast Utilities } \\
\text { Manchester, NH } \\
\text { office 603-634-2523 } \\
\text { lentips@nu.com }\end{array}$ \\
\hline Internal Reviewers & Naomi & Miller & $\begin{array}{l}\text { PNNL } \\
\text { Portland, OR } \\
\text { office 503-417-7571 } \\
\text { Naomi.Miller@pnl.gov }\end{array}$ \\
\hline Internal Team & Michael & Myer & $\begin{array}{l}\text { PNNL } \\
\text { Burlington, MA } \\
\text { office 781-685-4976 } \\
\text { michael.myer@pnl.gov }\end{array}$ \\
\hline Internal Team & Michn &
\end{tabular}





\section{Appendix B: Webtool Page Reference File}

\section{NOTE TO REVIEWERS:}

THANK YOU for taking the time to give us feedback on the Beta version of the Commercial Lighting Solutions webtool. We have provided a separate spreadsheet form for your use in capturing your comments (file name "CLS Spreadsheet Input Form 2010.xls"). In order for our team to process your comment, we need to locate it within the tool. Where applicable, please help us by listing the space type (e.g., Open Office, Corridor), the inputs used to determine applicable vignettes ( 8 '6" ceiling, $\left.5000 \mathrm{ft}^{2}\right)$, daylighting parameters (South, Light Shelves, Automatic Interior), as well as a reference webpage location name. Below you will see the names of the various pages within the tool.

Sincerely,

\section{The Commercial Lighting Solutions Team}

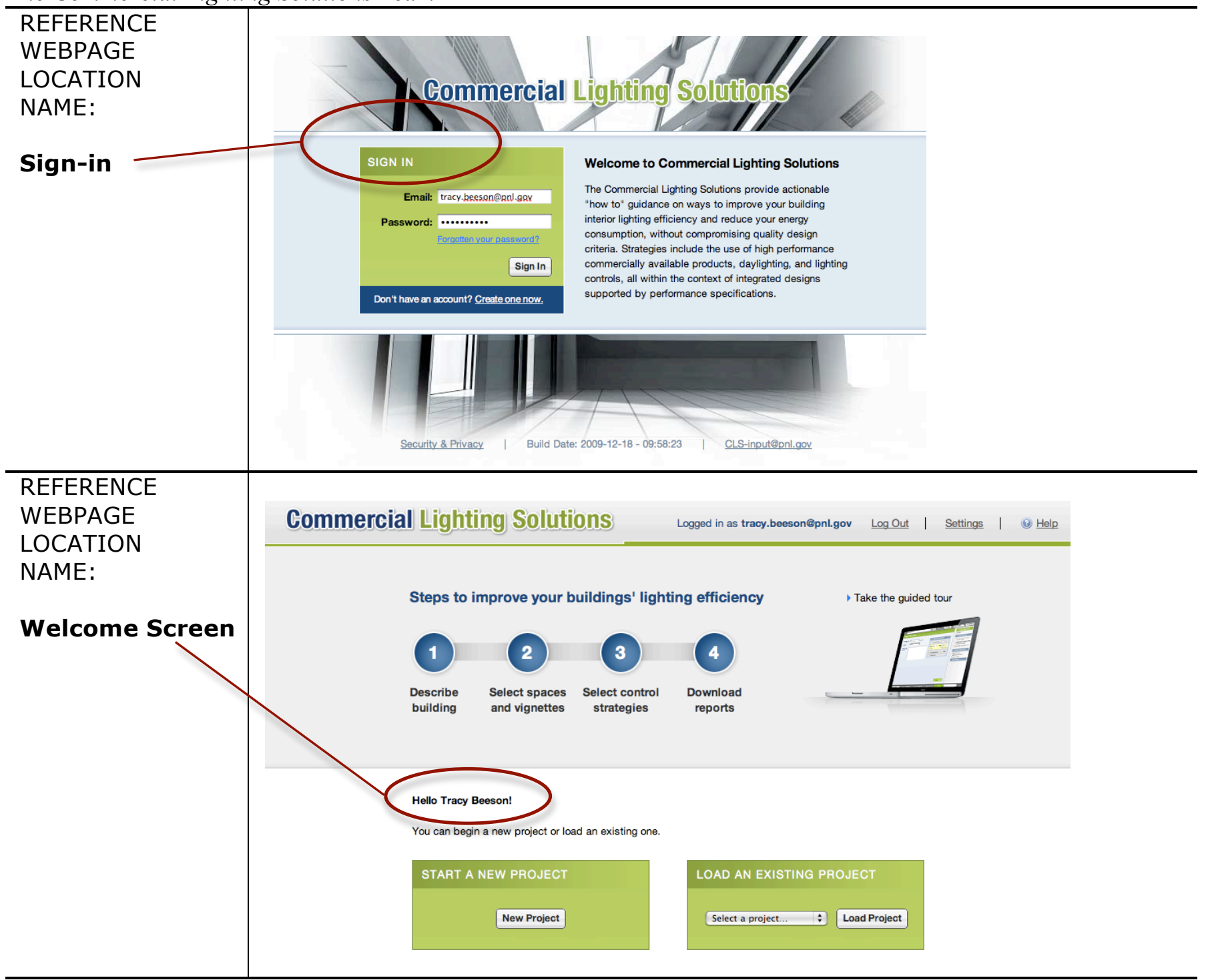




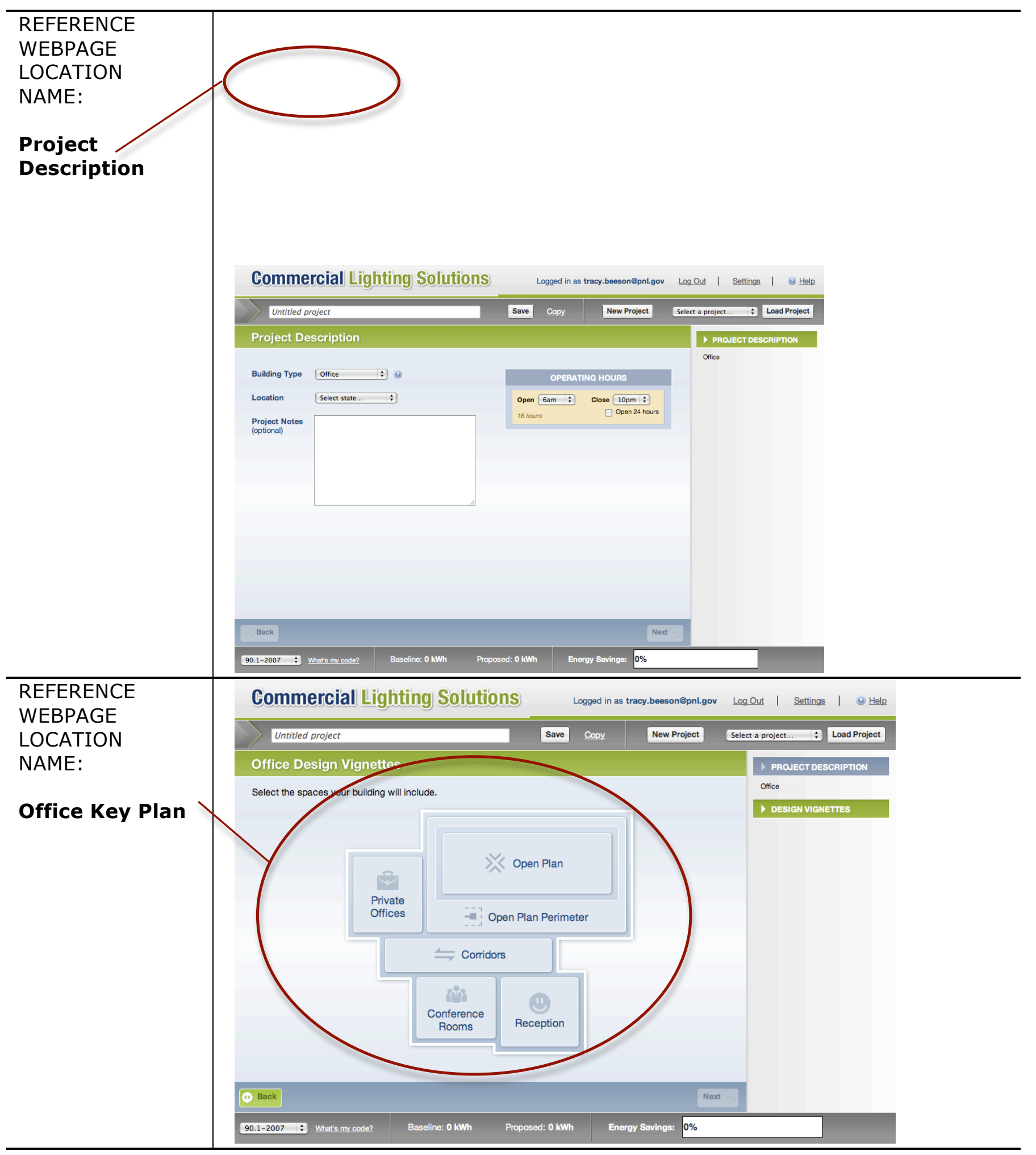

February 2010

Page B-2 


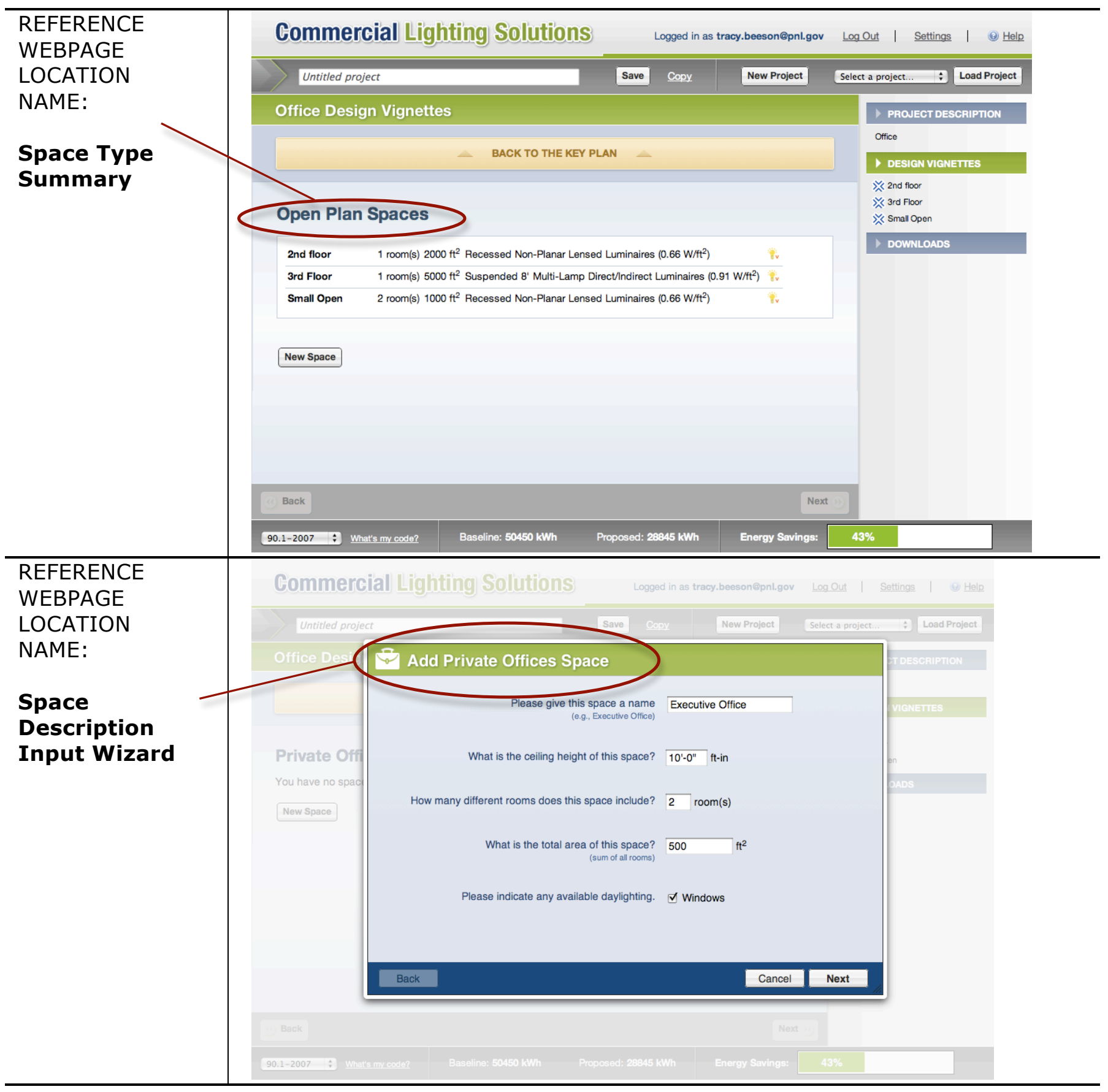




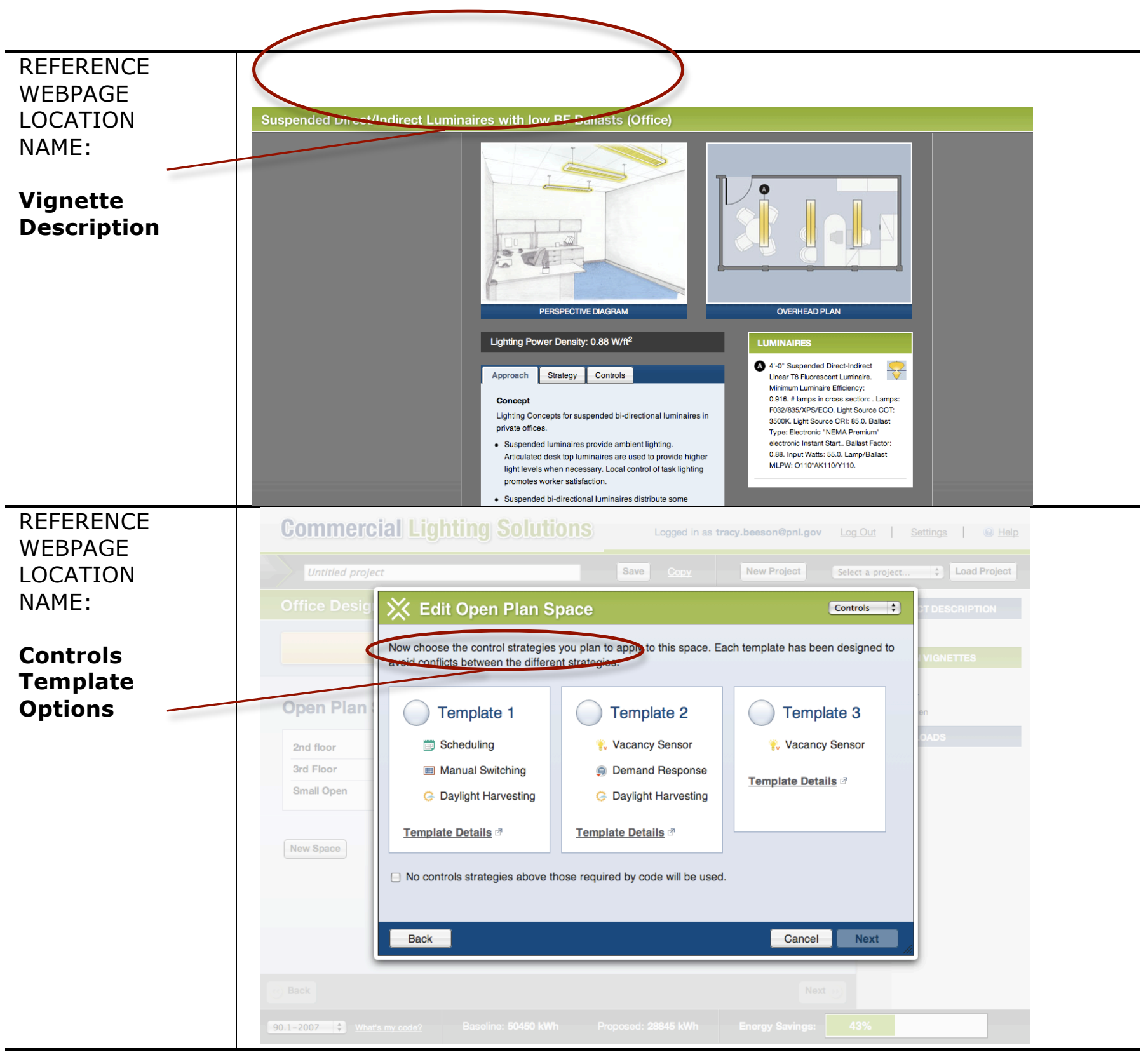




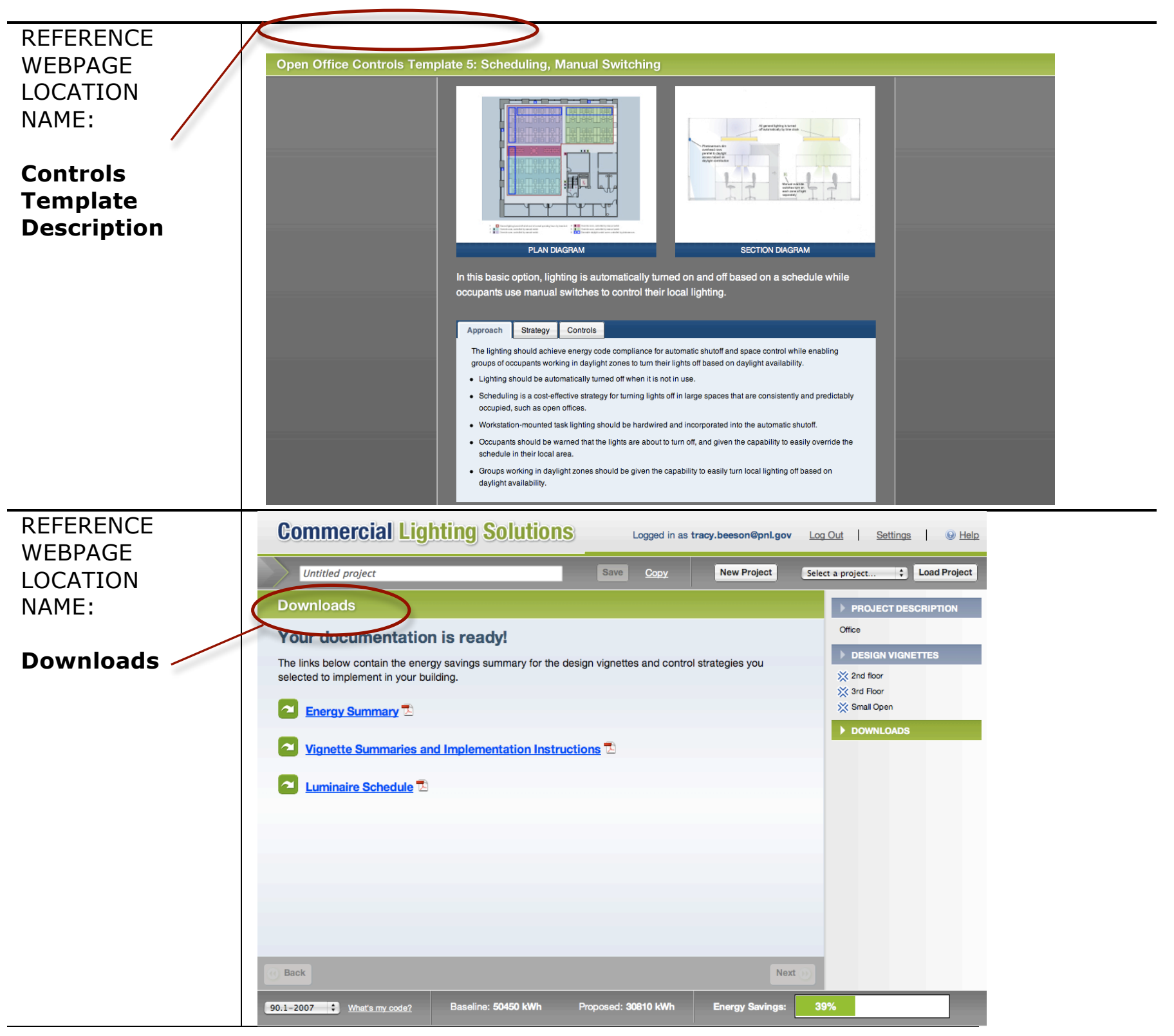





\section{Appendix C: Peer Review Input Spreadsheet}

Commercial Lighting Solutions, Peer Review Input Form

\begin{tabular}{|c|c|c|c|c|c|c|}
\hline Date: & & & & & COMMENT TYPES / QUESTIO & \\
\hline Commenter Name: & & & & & Functionality & Does the tool work the way it's supposed to? Are there bugs, problems? \\
\hline Organization Name: & & & & & Appearance/Aesthetics & Do you like the way it looks? \\
\hline Mac or PC: & & & & & Content & $\begin{array}{l}\text { Do you like the content of the tool, inclucing designs, text descriptions, graphics, luminaire } \\
\text { chart, etc? }\end{array}$ \\
\hline Operating System: & & & & & Ease of Use/Flow & Does the webtool lead you through the process well? Is it intulvere? \\
\hline Browser \& Version \#: & & & & & Download Materials & $\begin{array}{l}\text { Do you have feedback about the Energy Summary, Vignette Summaries and Implerrenation } \\
\text { Instructons of Luminaire Schedule? }\end{array}$ \\
\hline & & & & & General & Do you have any corments that apply to the whole tool or defy categorization? \\
\hline Comment Type & Space Type & $\begin{array}{l}\text { Ceiling Heighy } \\
\text { Total Area }\end{array}$ & $\begin{array}{l}\text { \# workstations/ } \\
\text { partition height }\end{array}$ & $\begin{array}{l}\text { Daylight Input (if } \\
\text { appoicable) }\end{array}$ & $\begin{array}{l}\text { Reference webpage or other } \\
\text { location (soe "Reference. doc) }\end{array}$ & Comment Descripticn: Issues, Suggestions \& Requests \\
\hline Example & Open Office & 875000 & 40060 & NorthWWMV100/2000.5.5 & Downloads & $\begin{array}{l}\text { I was not abie to seo tho Vignette Summary and implementation Instructions packet. Energy } \\
\text { Summary and Luminaire Schedule coened fine, but the midolio link was NG. }\end{array}$ \\
\hline & & & & & & \\
\hline & & & & & & \\
\hline & & & & & & \\
\hline & & & & & & \\
\hline & & & & & & \\
\hline & & & & & & \\
\hline & & & & & & \\
\hline & & & & & & \\
\hline & & & & & & \\
\hline & & & & & & \\
\hline & & & & & & \\
\hline & & & & & & \\
\hline & & & & & & \\
\hline TO ADO MORE ROWS, & EASE INSERI & ETHIS LINE. & & & & \\
\hline
\end{tabular}





\section{Appendix D: Ticket List}

\begin{tabular}{|c|c|c|c|}
\hline TICKET \# & PRIORITY & SUMMARY & DESCRIPTION/COMMENT \\
\hline 1 & A & $\begin{array}{l}\text { Incorporate definitions and } \\
\text { explanations of technical } \\
\text { information and acronyms }\end{array}$ & $\begin{array}{l}\text { Needs definitions, eliminate use of } \\
\text { acronyms, explain unknown terms or those } \\
\text { that have not yet been widely adopted }\end{array}$ \\
\hline 2 & A & $\begin{array}{l}\text { Verify vignette offerings } \\
\text { and applicability }\end{array}$ & $\begin{array}{l}\text { "Why are undercabinet fixtures not } \\
\text { recommended for my space with } 48 \text { " } \\
\text { partitions?" "Does uniformity of wall } \\
\text { mean across wall length; top to bottom?" } \\
\text { "Why is the LPD so high/low?" }\end{array}$ \\
\hline 3 & A & $\begin{array}{l}\text { Verify luminaire/lamp } \\
\text { names }\end{array}$ & $\begin{array}{l}\text { "The title of the luminaire says } \\
\text { direct/indirect, yet the distribution shows } \\
\text { more up than down. I would change the } \\
\text { title to indirect/direct," omit manufacturer } \\
\text { specific coding. "What exactly do you } \\
\text { mean by non-planar? Perhaps you should } \\
\text { call it "volumetric" type luminaires." }\end{array}$ \\
\hline 4 & A & $\begin{array}{l}\text { Review luminaire and } \\
\text { lamp data and confirm } \\
\text { descriptions sync with } \\
\text { calculations and } \\
\text { descriptive text }\end{array}$ & $\begin{array}{l}\text { "The LED undercabinet fixture should } \\
\text { produce more lumens than the articulated } \\
\text { desktop luminaire," verify luminaire } \\
\text { efficiencies, fill in blank information, } \\
\text { confirm MLPW (mean lumen per watt) } \\
\text { calculations }\end{array}$ \\
\hline 5 & A & $\begin{array}{l}\text { Integrate means of } \\
\text { communicating with user } \\
\text { when there are limited } \\
\text { vignette options for their } \\
\text { space parameters }\end{array}$ & Why is there only one vignette? \\
\hline 6 & A & $\begin{array}{l}\text { Resolve import issues that } \\
\text { cause incorrect characters } \\
\text { in the database }\end{array}$ & $\begin{array}{l}\text { Symbols for inches and dashes are } \\
\text { sometimes shown as question marks. }\end{array}$ \\
\hline 7 & A & $\begin{array}{l}\text { Add additional codes to } \\
\text { drop-down menu }\end{array}$ & $\begin{array}{l}\text { "Pennsylvania uses IECC } 2009 \text { it is not on } \\
\text { the list," and "I need title } 24 \text { baseline!" }\end{array}$ \\
\hline 9 & A & $\begin{array}{l}\text { Clarify workflow issues } \\
\text { with the user (i.e } \\
\text { navigating back to the key } \\
\text { plan, what "new space" } \\
\text { means, navigating to and } \\
\text { from vignette details) }\end{array}$ & $\begin{array}{l}\text { "I should always have a very legible, clear } \\
\text { path back to key plan; since the path link is } \\
\text { above where I'm working and not below, } \\
\text { where I would expect it to be, it should be } \\
\text { very legible," and "I found the Firefox } \\
\text { windows confusing, the program opens } \\
\text { new windows and I had trouble knowing } \\
\text { where the action was." }\end{array}$ \\
\hline 10 & A & $\begin{array}{l}\text { Modify the output from } \\
\text { the energy estimator in a } \\
\text { more flexible program that } \\
\text { can be more easily } \\
\text { modified (i.e. excel) }\end{array}$ & $\begin{array}{l}\text { "Exporting data to report across regions for } \\
\text { an entire portfolio is going to be helpful. } \\
\text { Need an electronic way to summarize } \\
\text { progress across the whole portfolio." }\end{array}$ \\
\hline
\end{tabular}




\begin{tabular}{|c|c|c|c|}
\hline TICKET \# & PRIORITY & SUMMARY & DESCRIPTION/COMMENT \\
\hline 11 & A & $\begin{array}{l}\text { Confirm control template } \\
\text { applicability and available } \\
\text { options based on the } \\
\text { vignette choice }\end{array}$ & $\begin{array}{l}\text { Should there be multi-level switching and } \\
\text { dimming in the same template, some users } \\
\text { want to see dimming or USL control in } \\
\text { private offices, provide more than } \\
\text { occupant switching for daylighting in each } \\
\text { vignette choice }\end{array}$ \\
\hline 12 & A & $\begin{array}{l}\text { Bring technical editor onto } \\
\text { the project and strive for } \\
\text { consistency across the } \\
\text { various content areas and } \\
\text { report outputs }\end{array}$ & $\begin{array}{l}\text { All text needs to be reviewed in the content } \\
\text { management system for accuracy and } \\
\text { agreement }\end{array}$ \\
\hline 13 & A & $\begin{array}{l}\text { Correct misinformation } \\
\text { and sizing issues } \\
\text { associated with the images }\end{array}$ & $\begin{array}{l}\text { Text too small on controls plans, } \\
\text { perspectives and RCPs have discrepancies, } \\
\text { luminaire labels wrong or missing }\end{array}$ \\
\hline 14 & A & $\begin{array}{l}\text { Improve controls template } \\
\text { titles so that they are more } \\
\text { meaningful to the user }\end{array}$ & $\begin{array}{l}\text { Remove back-end titles from showing up } \\
\text { on the UI or in the PDFs }\end{array}$ \\
\hline 16 & A & $\begin{array}{l}\text { Incorporate suggested } \\
\text { textual edits into the } \\
\text { controls template guidance }\end{array}$ & $\begin{array}{l}\text { Consider text edit recommendations and } \\
\text { implement if appropriate }\end{array}$ \\
\hline 17 & A & $\begin{array}{l}\text { Incorporate suggested } \\
\text { textual edits into the } \\
\text { lighting design vignettes }\end{array}$ & $\begin{array}{l}\text { Consider text edit recommendations and } \\
\text { implement if appropriate }\end{array}$ \\
\hline 18 & A & $\begin{array}{l}\text { Add a drop down menu } \\
\text { that gives users a sense of } \\
\text { window VT (visible } \\
\text { transmittance) if they do } \\
\text { not know offhand }\end{array}$ & $\begin{array}{l}\text { VT input potentially problematic, give } \\
\text { users an idea of what the potential ranges } \\
\text { are for different glazing types }\end{array}$ \\
\hline 19 & A & $\begin{array}{l}\text { Fix number of characters } \\
\text { associated with each } \\
\text { bullet- important } \\
\text { information is being lost }\end{array}$ & $\begin{array}{l}\text { UI routinely cutting off controls bulleted } \\
\text { information mid-sentence }\end{array}$ \\
\hline 20 & A & $\begin{array}{l}\text { Verify that energy } \\
\text { calculations are correct }\end{array}$ & $\begin{array}{l}\text { "When I got back to key plan, the energy } \\
\text { savings showed } 845 \% \text { savings. The } \\
\text { proposed savings showed NEGATIVE } \\
\text { kWh savings." }\end{array}$ \\
\hline 22 & A & $\begin{array}{l}\text { Expand luminaire lists to } \\
\text { account for different } \\
\text { ballast dimming } \\
\text { capabilities based on the } \\
\text { controls options specified }\end{array}$ & $\begin{array}{l}\text { Dimming ballasts not listed on luminaire } \\
\text { schedule, and instant start ballasts are } \\
\text { being shown, even though dimming was } \\
\text { specified }\end{array}$ \\
\hline 23 & A & $\begin{array}{l}\text { Limit the significant digits } \\
\text { represented in the tool } \\
\text { regarding luminaire } \\
\text { specifications }\end{array}$ & $\begin{array}{l}\text { Power values, CRI, MLPW, should be } \\
\text { whole numbers }\end{array}$ \\
\hline 24 & A & $\begin{array}{l}\text { Include controls selections } \\
\text { in the energy summary } \\
\text { document per space type }\end{array}$ & $\begin{array}{l}\text { Controls summary has no information in } \\
\text { the energy summary }\end{array}$ \\
\hline
\end{tabular}




\begin{tabular}{|c|c|c|c|}
\hline TICKET \# & PRIORITY & SUMMARY & DESCRIPTION/COMMENT \\
\hline 25 & A & $\begin{array}{l}\text { Specify kWh per vignette } \\
\text { in energy summary }\end{array}$ & $\begin{array}{l}\text { Provide users with a more granular } \\
\text { overview of their estimated energy } \\
\text { installation }\end{array}$ \\
\hline 27 & A & $\begin{array}{l}\text { Clean up formatting issues } \\
\text { associated with energy } \\
\text { summary download and } \\
\text { provide enough } \\
\text { information for it to be a } \\
\text { succinct stand alone } \\
\text { document }\end{array}$ & $\begin{array}{l}\text { Download information being labeled, as } \\
\text { "retail office" should not have retail in the } \\
\text { title, "what is the basis for the energy } \\
\text { summary? When it is divorced from the } \\
\text { webtool input process, the context is } \\
\text { unknown" }\end{array}$ \\
\hline 28 & A & $\begin{array}{l}\text { Present the controls } \\
\text { guidance and information } \\
\text { in a clear and } \\
\text { straightforward manner in } \\
\text { the PDF, without } \\
\text { unnecessary repetition or } \\
\text { confusing multiple } \\
\text { references to the same } \\
\text { space type }\end{array}$ & $\begin{array}{l}\text { "CLS needs to change the impression that } \\
\text { it gives of lighting controls [in the } \\
\text { downloads], which is an add-on to the } \\
\text { main event. Controls are the main event." }\end{array}$ \\
\hline 29 & A & $\begin{array}{l}\text { Clean up formatting issues } \\
\text { associated with } \\
\text { implementation and } \\
\text { summary downloads }\end{array}$ & $\begin{array}{l}\text { Controls images currently supported by } \\
\text { irrelevant captions, and sections are not } \\
\text { structured intuitively }\end{array}$ \\
\hline 30 & A & $\begin{array}{l}\text { Consider the file names } \\
\text { designated for the } \\
\text { download information, and } \\
\text { insure the files are being } \\
\text { called out and named in a } \\
\text { manner that is } \\
\text { communicative with the } \\
\text { user }\end{array}$ & $\begin{array}{l}\text { Report naming recommendations came } \\
\text { from multiple reviewers; discuss file } \\
\text { download naming and change if merited }\end{array}$ \\
\hline 31 & A & $\begin{array}{l}\text { Clean up formatting issues } \\
\text { associated with the } \\
\text { luminaire schedule } \\
\text { download }\end{array}$ & $\begin{array}{l}\text { "The luminaire report margins seemed off. } \\
\text { I think the report needs to be formatted." } \\
\text { Duplicate and resolve }\end{array}$ \\
\hline 32 & A & $\begin{array}{l}\text { Ensure that the } \\
\text { information from the user's } \\
\text { specific space shows up } \\
\text { correctly in the energy } \\
\text { estimator }\end{array}$ & $\begin{array}{l}\text { " Once again, in the energy summary you } \\
\text { need to specify individual room sizes and } \\
\text { quantity of rooms." }\end{array}$ \\
\hline 33 & A & $\begin{array}{l}\text { Include baseline code } \\
\text { controls in the database so } \\
\text { that the user does not get } \\
\text { error messages in the } \\
\text { energy estimator when not } \\
\text { going beyond code }\end{array}$ & $\begin{array}{l}\text { Users should have the option to apply } \\
\text { minimum code requirements to their } \\
\text { project if they choose }\end{array}$ \\
\hline
\end{tabular}




\begin{tabular}{|c|c|c|c|}
\hline TICKET \# & PRIORITY & SUMMARY & DESCRIPTION/COMMENT \\
\hline 35 & A & $\begin{array}{l}\text { Include explanation/clarify } \\
\text { algorithms in energy } \\
\text { estimator (or download) } \\
\text { for area of open office } \\
\text { perimeter spaces }\end{array}$ & $\begin{array}{l}\text { Wall-washing for open offices is calculated } \\
\text { on the back end, and the user needs to be } \\
\text { informed of the calculations and } \\
\text { assumptions that are being made to apply } \\
\text { the additional design to their space }\end{array}$ \\
\hline 39 & A & $\begin{array}{l}\text { Review retail vignettes for } \\
\text { any errors or inappropriate } \\
\text { design assumptions or } \\
\text { recommendations }\end{array}$ & $\begin{array}{l}\text { Should the aiming angle of luminaire be } \\
\text { mentioned, concerns over the installation } \\
\text { of vignettes for separate spaces not being } \\
\text { complimentary }\end{array}$ \\
\hline 42 & A & $\begin{array}{l}\text { Create or update any } \\
\text { images that are not current } \\
\text { (from retail) due to } \\
\text { changes in vignettes or re- } \\
\text { structuring of the image } \\
\text { paths with the inclusion of } \\
\text { offices }\end{array}$ & "Photos will not come in" \\
\hline 43 & A & $\begin{array}{l}\text { Ensure that users choose } \\
\text { their code baseline, and } \\
\text { the drop-down does not } \\
\text { have a default that allows } \\
\text { users to miss the box } \\
\text { entirely }\end{array}$ & $\begin{array}{l}\text { The drop down code menu isn't obvious } \\
\text { enough. Users would default to the wrong } \\
\text { code if they did not see it. }\end{array}$ \\
\hline 44 & A & $\begin{array}{l}\text { Change guided tour to be } \\
\text { inclusive of all of the new } \\
\text { features, and make sure } \\
\text { users know that it is video } \\
\text { and not static if their } \\
\text { speakers are not on }\end{array}$ & $\begin{array}{l}\text { The guided tour appeared to not be } \\
\text { working because the camera lingered on a } \\
\text { single screen while the narrator provided } \\
\text { an introduction, it also does not include a } \\
\text { tour for the energy estimator portion of the } \\
\text { tool }\end{array}$ \\
\hline 46 & A & $\begin{array}{l}\text { Clarify any confusion } \\
\text { associated with user input } \\
\text { requests }\end{array}$ & $\begin{array}{l}\text { Why do we require the information } \\
\text { requested? Wizard questions are not } \\
\text { entirely clear. Clarify between requests, } \\
\text { change language, etc. }\end{array}$ \\
\hline 47 & A & $\begin{array}{l}\text { Establish a standard with } \\
\text { mouse-overs by making it } \\
\text { part of the work-flow } \\
\text { process from the } \\
\text { beginning, or omitting the } \\
\text { use altogether } \\
\end{array}$ & $\begin{array}{l}\text { "This is the first time I saw a mouse-over } \\
\text { in the tool. This should be more obvious" }\end{array}$ \\
\hline 49 & A & $\begin{array}{l}\text { Modify the project } \\
\text { description page to be } \\
\text { building neutral until the } \\
\text { user chooses a building } \\
\text { type to avoid confusion, } \\
\text { modify operating hours } \\
\text { options to provide } \\
\text { necessary flexibility }\end{array}$ & $\begin{array}{l}\text { User did not think that offices were } \\
\text { included in the drop down when they saw } \\
\text { retail operating hour input on the screen } \\
\text { already. "I think that you can remove } 24 \\
\text { hour button for office buildings," } \\
\text { "Recommend having a weekend/weekday } \\
\text { feature," }\end{array}$ \\
\hline
\end{tabular}




\begin{tabular}{|c|c|c|c|}
\hline TICKET \# & PRIORITY & SUMMARY & DESCRIPTION/COMMENT \\
\hline 51 & A & $\begin{array}{l}\text { Improve the icons to be } \\
\text { more representative of the } \\
\text { space types/controls } \\
\text { strategies }\end{array}$ & $\begin{array}{l}\text { The vacancy sensor icon could be } \\
\text { improved, icon for multi-level switching } \\
\text { shows dimmers, not switches, etc. }\end{array}$ \\
\hline 52 & A & $\begin{array}{l}\text { Change luminaire } \\
\text { characteristics presentation } \\
\text { to read as a list rather than } \\
\text { bulleted form }\end{array}$ & $\begin{array}{l}\text { "Under the Luminaire tab I wonder if the } \\
\text { lighting characteristics would read easier if } \\
\text { the attributes were a bulleted list as } \\
\text { opposed to written in paragraph form." }\end{array}$ \\
\hline 53 & A & $\begin{array}{l}\text { Minimize the possibility of } \\
\text { users closing the tool } \\
\text { without saving progress } \\
\text { either by auto-save or } \\
\text { some sort of prompt }\end{array}$ & $\begin{array}{l}\text { "Needs auto-save or regular prompts to } \\
\text { save," "When I came back to the project } \\
\text { after having logged out, there was no } \\
\text { option for opening an existing project - } \\
\text { Perhaps I didn't save? the system should } \\
\text { ask before letting me exit." }\end{array}$ \\
\hline 54 & A & $\begin{array}{l}\text { Enlarge the size of the } \\
\text { "add space" box so that } \\
\text { input at the bottom is not } \\
\text { overlooked when users do } \\
\text { not see it (or) prevent user } \\
\text { from continuing until all } \\
\text { questions have been } \\
\text { acknowledged }\end{array}$ & $\begin{array}{l}\text { "When you hit next (because you can't see } \\
\text { the menu for VT until you scroll) it doesn't } \\
\text { let you go forward but I can't see why. } \\
\text { Can you enlarge the box?" }\end{array}$ \\
\hline 55 & A & $\begin{array}{l}\text { Change the space } \\
\text { summary page to make } \\
\text { editing or deleting spaces } \\
\text { more visible, and ensure } \\
\text { the correct units are being } \\
\text { reported }\end{array}$ & $\begin{array}{l}\text { "After finishing, the edit and delete icons } \\
\text { only come up when I scroll over them - } \\
\text { they should come up automatically" and } \\
\text { partition heights are listed feet, not inches }\end{array}$ \\
\hline 59 & A & $\begin{array}{l}\text { Resolve issues that give } \\
\text { users security warning } \\
\text { when navigating to the } \\
\text { website }\end{array}$ & $\begin{array}{l}\text { "Is there a way to add a security clearance } \\
\text { so that Firefox doesn't show the site as } \\
\text { untrusted?" }\end{array}$ \\
\hline 63 & $\mathrm{~A}$ & $\begin{array}{l}\text { All spaces should be } \\
\text { editable }\end{array}$ & $\begin{array}{l}\text { Make sure that spaces are editable without } \\
\text { users having to delete and re-add spaces }\end{array}$ \\
\hline 64 & A & $\begin{array}{l}\text { Project saving } \\
\text { functionality }\end{array}$ & $\begin{array}{l}\text { Resolve problems associated with re- } \\
\text { loading saved projects and users not being } \\
\text { able to save their project at all }\end{array}$ \\
\hline 65 & A & $\begin{array}{l}\text { Confirm user inputs are } \\
\text { being accepted as } \\
\text { requested }\end{array}$ & $\begin{array}{l}\text { "Despite the instructions to the contrary, } 0 \\
\text { is not an option for "no partitions" under } \\
\text { partition height. I ended up using } 1 \\
\text { instead." }\end{array}$ \\
\hline 66 & $\mathrm{~A}$ & $\begin{array}{l}\text { Clean up unintended } \\
\text { visuals }\end{array}$ & $\begin{array}{l}\text { "some weird text appears on the far left of } \\
\text { my } \\
\text { screen when I have the webpage take up } \\
\text { my entire screen" }\end{array}$ \\
\hline 67 & A & Resolve errors & $\begin{array}{l}\text { "When I clicked the new project button the } \\
\text { first time I got an error, the second time it } \\
\text { worked." }\end{array}$ \\
\hline
\end{tabular}




\begin{tabular}{|c|c|c|c|}
\hline TICKET \# & PRIORITY & SUMMARY & DESCRIPTION/COMMENT \\
\hline 68 & A & $\begin{array}{l}\text { Attempt to duplicate and } \\
\text { resolve missing and/or } \\
\text { reappearing elements of } \\
\text { the UI }\end{array}$ & $\begin{array}{l}\text { Red fields to fill in before continuing not } \\
\text { showing up, after adding ceiling ht. The } \\
\text { page format changed \# workstations box } \\
\text { moves }\end{array}$ \\
\hline 69 & A & $\begin{array}{l}\text { Space summary should } \\
\text { reflect user input }\end{array}$ & $\begin{array}{l}\text { Summary section not appropriately } \\
\text { reflecting user's input and did not change } \\
\text { after changes were made }\end{array}$ \\
\hline 70 & A & $\begin{array}{l}\text { Open plan perimeter drop- } \\
\text { down should function }\end{array}$ & $\begin{array}{l}\text { Drop down menu not listing applicable } \\
\text { open office space types; duplicate and } \\
\text { resolve }\end{array}$ \\
\hline 71 & A & Errors retrieving vignettes & $\begin{array}{l}\text { Duplicate and resolve problems associated } \\
\text { with vignette retrieval }\end{array}$ \\
\hline 8 & B & $\begin{array}{l}\text { Consider expanding space } \\
\text { types covered in the office } \\
\text { section of the tool and } \\
\text { offering more vignettes for } \\
\text { spaces with limited } \\
\text { options }\end{array}$ & $\begin{array}{l}\text { "2 typical spaces common in almost every } \\
\text { office application are a copy room and a } \\
\text { break room, they should be added to the } \\
\text { list of spaces." Only two corridor lighting } \\
\text { options- hope these are first because they } \\
\text { are the most common, not the only option }\end{array}$ \\
\hline 15 & B & $\begin{array}{l}\text { Provide users transparency } \\
\text { about the design } \\
\text { development, required } \\
\text { equipment, baseline } \\
\text { characteristics and } \\
\text { assumptions made }\end{array}$ & $\begin{array}{l}\text { Provide estimate of how much of the LPD } \\
\text { is from the desktop luminaires, explanation } \\
\text { of what baseline means, need for dimming } \\
\text { ballasts should be mentioned in controls } \\
\text { strategies that use dimming }\end{array}$ \\
\hline 21 & B & $\begin{array}{l}\text { Omit headings that do not } \\
\text { have any associated text } \\
\text { stored in the database }\end{array}$ & $\begin{array}{l}\text { Headings with no information and bullets } \\
\text { without text mislead the user by making } \\
\text { them think that information is missing }\end{array}$ \\
\hline 36 & B & $\begin{array}{l}\text { Communicate the } \\
\text { efficiency of the interior } \\
\text { design considerations in } \\
\text { the UI }\end{array}$ & $\begin{array}{l}\text { "No tab for interior design layouts or } \\
\text { strategies. The user should know this is the } \\
\text { least efficient layout before proceeding. } \\
\text { Information is } \\
\text { useless at the report printout stage." }\end{array}$ \\
\hline 37 & B & $\begin{array}{l}\text { Offer available vignettes } \\
\text { in order of lighting power } \\
\text { density }\end{array}$ & $\begin{array}{l}\text { "do we want to choose the order of } \\
\text { presentation of the vignettes? Prioritize by } \\
\text { LPD?" }\end{array}$ \\
\hline 40 & B & $\begin{array}{l}\text { Include vertical } \\
\text { illumination ranges for the } \\
\text { display lighting from } \\
\text { general lighting }\end{array}$ & $\begin{array}{l}\text { Vertical illuminance is even more } \\
\text { important than horizontal illuminance for } \\
\text { retail display; we should inform users what } \\
\text { our models yield }\end{array}$ \\
\hline 41 & B & $\begin{array}{l}\text { Clarify any existing points } \\
\text { of confusion in the retail } \\
\text { vignettes }\end{array}$ & $\begin{array}{l}\text { There is no information as to why } \\
\text { luminaires are off-center in some vignettes, } \\
\text { users unclear about how LPD is broken } \\
\text { down by space type, application of lighting } \\
\text { controls is unclear }\end{array}$ \\
\hline 50 & B & $\begin{array}{l}\text { Provide an "I don't know" } \\
\text { option for users that do not } \\
\text { know their furniture layout } \\
\text { or daylighting parameters }\end{array}$ & $\begin{array}{l}\text { "I don't know what the size of the window } \\
\text { openings are." and "what if you don't have } \\
\text { your furniture plan layout, can you answer } \\
\text { with a "0" or N/A?" }\end{array}$ \\
\hline
\end{tabular}




\begin{tabular}{|c|c|c|c|}
\hline TICKET \# & PRIORITY & SUMMARY & DESCRIPTION/COMMENT \\
\hline 56 & B & $\begin{array}{l}\text { Enhance control tab to } \\
\text { provide more detailed and } \\
\text { applicable information }\end{array}$ & $\begin{array}{l}\text { Controls tab is missing icons and } \\
\text { explanations of what the different controls } \\
\text { strategies are }\end{array}$ \\
\hline 61 & B & $\begin{array}{l}\text { Provide more flexibility } \\
\text { for users when inputting } \\
\text { space area (i.e. allowing } \\
\text { them to input area and } \\
\text { number of rooms, } \\
\text { dimensions, or provide } \\
\text { some sort of space } \\
\text { calculator) }\end{array}$ & $\begin{array}{l}\text { "Instead of having me do the math for the } \\
\text { total area, can you put in SF per each of } \\
\text { private offices?" and "have ability to but in } \\
\text { dimensions and have program calculate sq } \\
\mathrm{ft"}\end{array}$ \\
\hline 34 & $\mathrm{C}$ & $\begin{array}{l}\text { Include percentage savings } \\
\text { in watts per square foot in } \\
\text { the energy estimator } \\
\text { download information to } \\
\text { facilitate LEED } \\
\text { compliance }\end{array}$ & $\begin{array}{l}\text { "LEED credits don't allow for kWh, so we } \\
\text { need to also show the LEED savings, } \\
\text { percentage of savings in w/SF" }\end{array}$ \\
\hline 48 & $\mathrm{C}$ & $\begin{array}{l}\text { Make controls choices } \\
\text { more apparent in the space } \\
\text { summary }\end{array}$ & $\begin{array}{l}\text { Symbols are not legible in the space } \\
\text { summary, and controls choices should be } \\
\text { more prominently displayed }\end{array}$ \\
\hline 57 & $\mathrm{C}$ & $\begin{array}{l}\text { Change titles within the UI } \\
\text { to help users better } \\
\text { understand where they are } \\
\text { in the work flow process }\end{array}$ & $\begin{array}{l}\text { "Recommend changing the heading of this } \\
\text { window to say controls" and "Recommend } \\
\text { changing the heading of this window to say } \\
\text { daylighting" }\end{array}$ \\
\hline 58 & $\mathrm{C}$ & $\begin{array}{l}\text { Provide users with } \\
\text { guidance on how to } \\
\text { choose quality products } \\
\text { that will provide the } \\
\text { results in the lighting } \\
\text { system that the tool claims }\end{array}$ & $\begin{array}{l}\text { "How do you deal with the luminaire } \\
\text { efficiency and photometric performance? } \\
\text { Not all suspended direct/indirects are going } \\
\text { to give you these results." }\end{array}$ \\
\hline 60 & $\mathrm{C}$ & $\begin{array}{l}\text { Include information so that } \\
\text { users know where the } \\
\text { majority of the savings are } \\
\text { coming from and how } \\
\text { effective their controls } \\
\text { strategies are without } \\
\text { burdening them with more } \\
\text { questions or providing too } \\
\text { much detail as to } \\
\text { overwhelm }\end{array}$ & $\begin{array}{l}\text { Is there a way to relate to users the energy } \\
\text { savings that can be attributed to the } \\
\text { lighting system or the controls system? } \\
\text { Integrating into the UI is very challenging, } \\
\text { but some level of detail could be integrated } \\
\text { into the report. The level of detail is TBD, } \\
\text { since it can be dangerous to accommodate } \\
\text { the picking and choosing of which } \\
\text { elements of the lighting solution to apply, } \\
\text { since they are designed as full solution sets }\end{array}$ \\
\hline 62 & $\mathrm{C}$ & $\begin{array}{l}\text { Flag equipment that is not } \\
\text { high performance }\end{array}$ & $\begin{array}{l}\text { "Can you put in a notes field to flag } \\
\text { equipment that isn't high performance?" }\end{array}$ \\
\hline
\end{tabular}



Appendix E: Master Comments List

\begin{tabular}{|c|c|c|c|c|c|c|c|c|c|c|c|c|c|c|c|c|c|c|c|c|}
\hline $\begin{array}{l}\text { Commenter } \\
\text { ID \# }\end{array}$ & $\begin{array}{l}\text { Comment } \\
\text { Reference } \\
\#\end{array}$ & $\begin{array}{l}\text { Commenter } \\
\text { Category }\end{array}$ & $\begin{array}{l}\text { Comment } \\
\text { Type }\end{array}$ & Space Type & \begin{tabular}{|l|} 
Ceiling \\
Height/ \\
Total Area
\end{tabular} & \begin{tabular}{|l|} 
\\
workstations/ \\
partition \\
height (open \\
office only)
\end{tabular} & $\mid \begin{array}{l}\text { Daylight } \\
\text { Input (if } \\
\text { applicable) }\end{array}$ & \begin{tabular}{|l|} 
Reference webpage \\
or other location \\
(see \\
"Reference.doc") \\
\end{tabular} & $\begin{array}{l}\text { Comment Description: } \\
\text { Issues, Suggestions \& } \\
\text { Requests }\end{array}$ & Action/Solution & \begin{tabular}{|l|} 
Status \\
or \\
Ticket \#
\end{tabular} & $\begin{array}{c}\text { Frequenc } \\
\mathrm{y}(20 \%)\end{array}$ & $\begin{array}{c}\text { Importance } \\
\text { of comment } \\
(80 \%)\end{array}$ & $\begin{array}{c}\text { Sum } \\
(100 \%)\end{array}$ & $\begin{array}{c}\text { Ease of } \\
\text { Implementatio } \\
\mathrm{n}(30 \%)\end{array}$ & $\begin{array}{c}\text { Duration } \\
(40 \%)\end{array}$ & $\begin{array}{c}\text { Risk/ } \\
\text { Complicatio } \\
\mathrm{n}(30 \%)\end{array}$ & \begin{tabular}{|} 
Sum \\
$(100 \%)$
\end{tabular} & \begin{tabular}{|c|}
$\begin{array}{c}\text { Short Term } \\
(40 \% \\
\text { Importance) } \\
(60 \% \text { Feasibility) }\end{array}$ \\
\end{tabular} & $\begin{array}{c}\text { Long Term } \\
(85 \% \\
\text { Importance) } \\
\text { (15\% Feasibility) }\end{array}$ \\
\hline$\sqrt{J \text { Beutell }}$ & JB7 & Owner & & & & & & & \begin{tabular}{|l|} 
How do you deal with the \\
luminaire efficiency and \\
photometric performance? \\
Not all suspended \\
directindirects are going to \\
give you these results. \\
\end{tabular} & Additions & 58 & 3 & 5 & 4.6 & 1 & 1 & 3 & 1.6 & 3.4 & 4.2 \\
\hline R Parish & RP4 & Owner & & & & & & & $\begin{array}{l}\text { Exporting data to report } \\
\text { across regions for an entire } \\
\text { portfolio is going to be } \\
\text { helpful. Need an electronic } \\
\text { way to summarize progress } \\
\text { across the whole portfolio. }\end{array}$ & Additions & 10 & 2 & 5 & 4.4 & 4 & 3 & 5 & 3.9 & 4.2 & 4.3 \\
\hline R Parish & RP3 & Owner & & & & & & & \begin{tabular}{|l|} 
Clients that have portfolios \\
in multiple locations.
\end{tabular} & Additions & 10 & 2 & 5 & 4.4 & 4 & 3 & 5 & 3.9 & 4.2 & 4.3 \\
\hline \begin{tabular}{|l|} 
A Cortese \\
\end{tabular} & AC7 & Architect & $\begin{array}{l}\text { Ease of } \\
\text { Use/Flow }\end{array}$ & & & & VT & & \begin{tabular}{|l|} 
it seems unlikely that this \\
will actually be known, \\
except for new \\
construction. Is there some \\
guidance that you can \\
provide to ensure this is \\
estimated correctly? (i.e. \\
smoky gray glass is 0.3, \\
etc). Also, please note that \\
1 , which is impossible to \\
achieve, is the only input \\
acceptable by the system.
\end{tabular} & Additions & 18 & 3 & 5 & 4.6 & 4 & 3 & 4 & 3.6 & 4.2 & 4.5 \\
\hline HMcKay & HM14 & Designer & Functionality & & & & & & \begin{tabular}{|l|} 
VLT input box will not \\
accept. 3 - it must have a \\
zero in front of it: 0.3
\end{tabular} & Additions & 18 & 3 & 5 & 4.6 & 4 & 3 & 4 & 3.6 & 4.2 & 4.5 \\
\hline A Cortese & AC24 & Architect & Functionality & & & & & & \begin{tabular}{|l|} 
Visual Transmittance - The \\
only VT number that I could \\
input was 1, which is not \\
technically possible. \\
\end{tabular} & Additions & 18 & 3 & 5 & 4.6 & 5 & 5 & 5 & 5 & 4.8 & 4.7 \\
\hline \begin{tabular}{|l}
$J$ Manierre \\
\end{tabular} & JJM2 & Utility & & & & & & \begin{tabular}{|l} 
Jennifer Manierre, \\
NYSERDA
\end{tabular} & \begin{tabular}{|l|} 
Input wattages are going to \\
vary per program. How do \\
we deal with this??? From \\
device list.
\end{tabular} & Content & TBD & 1 & 3 & 2.6 & 2 & 2 & 3 & 2.3 & 2.5 & 2.6 \\
\hline $\begin{array}{l}\text { C Magee } \\
\text {. }\end{array}$ & CM10 & Owner & & & & & & & $\begin{array}{l}\text { Hotel rooms can be very } \\
\text { consistent, would be a } \\
\text { good space type to offer in } \\
\text { the future. }\end{array}$ & Content & LT & 1 & 4 & 3.4 & 2 & 1 & 3 & 1.9 & 2.8 & 3.2 \\
\hline $\begin{array}{l}\text { C Magee } \\
\text {. }\end{array}$ & CM11 & Owner & & & & & & & \begin{tabular}{|l|} 
For the future, once the \\
HVAC tool is build, I'd \\
suggest keeping kWh \\
savings separate from \\
combined HVAC savings. \\
CFO's, finance folks, don't \\
always accept the ancillary \\
savings that are promised.
\end{tabular} & Content & TBD & 1 & 4 & 3.4 & 2 & 1 & 3 & 1.9 & 2.8 & 3.2 \\
\hline M Myer & MM285 & Internal & Content & $\begin{array}{l}\text { Recessed 4' } \\
\text { Asymmetric } \\
\text { wall-wash } \\
\text { luminaires }\end{array}$ & & & \begin{tabular}{|l|} 
corridors \\
\end{tabular} & Approach/Criteria & $\begin{array}{l}\text { CCT issue with this text } \\
\text { and the luminaire box }\end{array}$ & Content & 4 & 1 & 3 & 2.6 & 3 & 3 & 4 & 3.3 & 2.9 & 2.7 \\
\hline
\end{tabular}


Appendix E: Master Comments List

\begin{tabular}{|c|c|c|c|c|c|c|c|c|c|c|c|c|c|c|c|c|c|c|c|c|}
\hline $\begin{array}{l}\text { Commenter } \\
\text { ID \# }\end{array}$ & $\begin{array}{l}\text { Comment } \\
\text { Reference } \\
\#\end{array}$ & $\begin{array}{l}\text { Commenter } \\
\text { Category }\end{array}$ & \begin{tabular}{|l} 
Comment \\
Type
\end{tabular} & Space Type & \begin{tabular}{|l|} 
Ceiling \\
Height/ \\
Total Area
\end{tabular} & \begin{tabular}{|l}
$\#$ \\
workstations/ \\
partition \\
height (open \\
office only)
\end{tabular} & $\begin{array}{l}\text { Daylight } \\
\text { Input (if } \\
\text { applicable) }\end{array}$ & \begin{tabular}{|l|} 
Reference webpage \\
or other location \\
(see \\
"Reference.doc") \\
\end{tabular} & $\begin{array}{l}\text { Comment Description: } \\
\text { Issues, Suggestions \& } \\
\text { Requests }\end{array}$ & Action/Solution $\left[\begin{array}{l}5 \\
0 \\
T\end{array}\right.$ & \begin{tabular}{|l|} 
Status \\
or \\
Ticket \#
\end{tabular} & $\begin{array}{l}\text { Frequenc } \\
\mathrm{y}(20 \%)\end{array}$ & $\begin{array}{c}\text { Importance } \\
\text { of comment } \\
(80 \%)\end{array}$ & $\begin{array}{c}\text { Sum } \\
(100 \%)\end{array}$ & $\begin{array}{c}\text { Ease of } \\
\text { Implementatio } \\
\mathrm{n}(30 \%)\end{array}$ & $\begin{array}{c}\text { Duration } \\
(40 \%)\end{array}$ & \begin{tabular}{|c} 
Risk/ \\
Complicatio \\
$\mathrm{n}(30 \%)$
\end{tabular} & \begin{tabular}{|} 
Sum \\
$(100 \%)$
\end{tabular} & \begin{tabular}{|c|} 
Short Term \\
$(40 \%$ \\
Importance) \\
(60\% Feasibility)
\end{tabular} & $\begin{array}{c}\text { Long Term } \\
\text { (85\% } \\
\text { Importance) } \\
\text { (15\% Feasibility) } \\
\end{array}$ \\
\hline W Dau & WD12 & $\begin{array}{l}\text { Designer/IES } \\
\text { Chair }\end{array}$ & & & & & & & $\begin{array}{l}2 \text { typical spaces common in } \\
\text { almost every office } \\
\text { application are a copy room } \\
\text { and a break room, they } \\
\text { should be added to the list } \\
\text { of spaces. }\end{array}$ & Content & 8 & 1 & 4 & 3.4 & 2 & 2 & 3 & 2.3 & 3.0 & 3.2 \\
\hline J Yorgey & JY37 & Manufacturer & General & & & & & Overall & $\begin{array}{l}\text { Not enough fixture type } \\
\text { selections }\end{array}$ & Content & 8 & 1 & 4 & 3.4 & 2 & 2 & 4 & 2.6 & 3.1 & 3.3 \\
\hline M Myer & MM575 & Internal & Content & $\begin{array}{l}\text { Suspended 8' } \\
\text { multi-lamp } \\
(0.69 \text { W/sf })\end{array}$ & $10 / 5000$ & $50 / 48$ & & $\begin{array}{l}\text { Approach/Luminaire } \\
\text { s }\end{array}$ & \begin{tabular}{|l|} 
I think that we should \\
provide an estimate of how \\
much of the LPD is from \\
the desktop luminaires
\end{tabular} & Content & 15 & 2 & 4 & 3.6 & 2 & 3 & 2 & 2.4 & 3.1 & 3.4 \\
\hline M Myer & MM284 & Internal & Content & $\begin{array}{l}\text { Recessed 4' } \\
\text { Asymmetric } \\
\text { wall-wash } \\
\text { luminaires }\end{array}$ & & & corridors & Approach/Criteria & \begin{tabular}{|l|} 
The vertical illuminance on \\
at least one wall...Can that \\
be the short end wall? Do \\
you want it to be along on \\
of the long-axis walls?
\end{tabular} & Content & 2 & 1 & 4 & 3.4 & 3 & 3 & 3 & 3 & 3.2 & 3.3 \\
\hline \begin{tabular}{|l|l|}
$J$ Manierre \\
\end{tabular} & $\mid \mathrm{JM} 3$ & Utility & & & & & & \begin{tabular}{|l} 
Jennifer Manierre, \\
NYSERDA
\end{tabular} & $\begin{array}{l}\text { What do we do with } \\
\text { equipment that isn't } \\
\text { recommended? }\end{array}$ & Content & 62 & 2 & 4 & 3.6 & 3 & 3 & 2 & 2.7 & 3.2 & 3.5 \\
\hline M Dare & MD9 & Utility & & & & & & & \begin{tabular}{|l|} 
How are you going to \\
characterize the glare \\
issues that can come with \\
"non-planar" type fixutres? \\
Some are OK, and some \\
are not. You should note \\
that none of these fixtures \\
meet RP-1.
\end{tabular} & Content & 58 & 3 & 5 & 4.6 & 1 & 1 & 3 & 1.6 & 3.4 & 4.2 \\
\hline M Myer & MM329 & Internal & Content & \begin{tabular}{|l|} 
Suspended \\
Direct/lndirect \\
Luminaires with \\
low BF ballasts
\end{tabular} & $9^{\prime}$ & $\mathrm{PO}$ & & Title & \begin{tabular}{|l|} 
The title of the lumianire \\
says direct/indirect, yet the \\
distribution shows more up \\
than down. I would change \\
the title to indirect/direct
\end{tabular} & Content & 3 & 1 & 4 & 3.4 & 4 & 3 & 4 & 3.6 & 3.5 & 3.4 \\
\hline M Myer & MM550 & Internal & Content & $\begin{array}{l}\text { Suspended 8' } \\
\text { multi-lamp } \\
(0.69 \mathrm{~W} / \mathrm{sf})\end{array}$ & $10 / 5000$ & $50 / 48$ & & Luminaires B & \begin{tabular}{|l} 
I recommend changing \\
"Direct-Indirect" to \\
"Indirect/Direct"
\end{tabular} & Content & 3 & 1 & 4 & 3.4 & 4 & 3 & 4 & 3.6 & 3.5 & 3.4 \\
\hline M Myer & MM291 & Internal & Content & $\begin{array}{l}\text { Recessed 4' } \\
\text { Asymmetric } \\
\text { wall-wash } \\
\text { luminaires }\end{array}$ & & & corridors & \begin{tabular}{|l|} 
Strategy/Maintenan \\
ce
\end{tabular} & $\begin{array}{l}5 \% \text { lumen depreciation is } \\
\text { aggressive. Also over the } \\
\text { rated life of the lamp is } \\
\text { different than the mean } \\
\text { lumen value. }\end{array}$ & Content & 4 & 3 & 4 & 3.8 & 3 & 3 & 3 & 3 & 3.5 & 3.7 \\
\hline M Myer & \begin{tabular}{|l|} 
MM493 \\
\end{tabular} & Internal & Content & \begin{tabular}{|l|} 
Recessed Non- \\
Planar (LPD \\
0.64 W/sf) \\
\end{tabular} & $8 / 5000$ & $30 / 48$ & & Luminaire choices & $\begin{array}{l}\text { Why are LED undercabinet } \\
\text { fixtures recommended } \\
\text { here, but not for another } \\
\text { design with } 48 \text { " tall } \\
\text { partitions and a ceiling } \\
\text { height of } 9 \text { '-6". I do not } \\
\text { think that ceiling height is } \\
\text { the sole driving issue for } \\
\text { LED undercabinet fixtures }\end{array}$ & Content & 2 & 1 & 5 & 4.2 & 2 & 3 & 3 & 2.7 & 3.6 & 4.0 \\
\hline N Miller & NM20 & Internal & & & & & & & \begin{tabular}{|l|} 
General question: I \\
assume that the small \\
number of options in this \\
example doesn't mean that \\
recessed open slot T8 \\
downlights are the only \\
electric lighting solution. I \\
bet this is just the first one \\
because it's the most \\
common.
\end{tabular} & Content & 8 & 2 & 4 & 3.6 & 4 & 3 & 4 & 3.6 & 3.6 & 3.6 \\
\hline
\end{tabular}


Appendix E: Master Comments List

\begin{tabular}{|c|c|c|c|c|c|c|c|c|c|c|c|c|c|c|c|c|c|c|c|c|}
\hline $\begin{array}{l}\text { Commenter } \\
\text { ID \# }\end{array}$ & $\begin{array}{l}\text { Comment } \\
\text { Reference } \\
\#\end{array}$ & $\begin{array}{l}\text { Commenter } \\
\text { Category }\end{array}$ & \begin{tabular}{|l} 
Comment \\
Type
\end{tabular} & Space Type & \begin{tabular}{|l|} 
Ceiling \\
Height/ \\
Total Area
\end{tabular} & \begin{tabular}{|l}
$\#$ \\
workstations/ \\
partition \\
height (open \\
office only)
\end{tabular} & \begin{tabular}{|l|} 
Daylight \\
Input (if \\
applicable)
\end{tabular} & $\begin{array}{l}\text { Reference webpage } \\
\text { or other location } \\
\text { (see } \\
\text { "Reference.doc") }\end{array}$ & $\begin{array}{l}\text { Comment Description: } \\
\text { Issues, Suggestions \& } \\
\text { Requests }\end{array}$ & Action/Solutio & \begin{tabular}{|l|} 
Status \\
or \\
Ticket \#
\end{tabular} & $\begin{array}{c}\text { Frequenc } \\
\mathrm{y}(20 \%)\end{array}$ & $\begin{array}{c}\text { Importance } \\
\text { of comment } \\
(80 \%)\end{array}$ & \begin{tabular}{|c} 
Sum \\
$(100 \%)$
\end{tabular} & $\begin{array}{c}\text { Ease of } \\
\text { Implementatio } \\
\mathrm{n}(30 \%)\end{array}$ & $\begin{array}{c}\text { Duration } \\
(40 \%)\end{array}$ & $\begin{array}{c}\text { Risk/ } \\
\text { Complicatio } \\
\mathrm{n}(30 \%)\end{array}$ & \begin{tabular}{|c|} 
Sum \\
$(100 \%)$
\end{tabular} & \begin{tabular}{|c|} 
Short Term \\
$(40 \%$ \\
Importance) \\
(60\% Feasibility)
\end{tabular} & $\begin{array}{c}\text { Long Term } \\
\text { (85\% } \\
\text { Importance) } \\
\text { (15\% Feasibility) } \\
\end{array}$ \\
\hline N Miller & NM17 & Internal & & & & & & & \begin{tabular}{|l|}
. I assume "uniformity of \\
luminance" means \\
"uniformity of wall \\
luminance from top to \\
bottom of wall". Or does it \\
mean across the entire wall \\
length, top to bottom? \\
\end{tabular} & Content & 2 & 1 & 4 & 3.4 & 4 & 4 & 4 & 4 & 3.6 & 3.5 \\
\hline M Myer & MM517 & Internal & Content & \begin{tabular}{|l|} 
Recessed Non- \\
Planar (LPD \\
0.64 W/sf) \\
\end{tabular} & $8 / 5000$ & $30 / 48$ & & Luminaires C & \begin{tabular}{|l|} 
Input watts of 9.2 seems \\
low. How big is this fixture?
\end{tabular} & Content & 4 & 1 & 4 & 3.4 & 4 & 4 & 4 & 4 & 3.6 & 3.5 \\
\hline \begin{tabular}{|l|} 
D Maniccia \\
\end{tabular} & DM14 & Controls & & & & & & & \begin{tabular}{|l|} 
There should a way to \\
show the baseline LPD on \\
the summary key plan \\
screen once the savings \\
starts to show up. There \\
should be a way to explain \\
what baseline includes, \\
e.g., baseline kWh = code \\
compliant, which does \\
include both LPD allowed \\
in code and the required \\
controls. \\
\end{tabular} & Content & 15 & 1 & 4 & 3.4 & 4 & 4 & 4 & 4 & 3.6 & 3.5 \\
\hline M Myer & MM580 & Internal & Content & \begin{tabular}{|l|} 
Perimter \\
Recessed CFLs
\end{tabular} & & & & LPD & $\begin{array}{l}\text { Why is the LPD so high? If } \\
\text { the fixtures are only } 28 \mathrm{~W} \\
\text { and the LPD is } 0.97 \mathrm{~W} / \mathrm{sf}, \\
\text { then this design is } \\
\text { assuming there is one } \\
\text { fixture per every } 29 \mathrm{sq} \text { feet. } \\
\text { Is that correct? }\end{array}$ & Content & 2 & 1 & 4 & 3.4 & 4 & 4 & 5 & 4.3 & 3.8 & 3.5 \\
\hline HMcKay & HM37 & Designer & Content & & & & & & $\begin{array}{l}\text { V1- Title of vignette says } \\
\text { "low BF ballasts", but } \\
\text { descriptions, strategy and } \\
\text { sidebar shows standard BF } \\
\text { of .88. }\end{array}$ & Content & 4 & 1 & 4 & 3.4 & 4 & 4 & 5 & 4.3 & 3.8 & 3.5 \\
\hline M Myer & MM590 & Internal & Content & \begin{tabular}{|l|} 
Perimter \\
Recessed CFLs
\end{tabular} & & & & Luminaires & $\begin{array}{l}\text { I think that } \mathrm{BF} \text { is wrong, it is } \\
\text { probably } 0.98 \text { or } 1.02\end{array}$ & Content & 4 & 1 & 4 & 3.4 & 4 & 4 & 5 & 4.3 & 3.8 & 3.5 \\
\hline M Myer & MM185 & Internal & Content & \begin{tabular}{|l|} 
Recessed slot \\
luminaires \\
Perpendicular \\
to Desk with \\
Compact \\
Fluorescent \\
Downlights and \\
Wall-washers \\
(Office)
\end{tabular} & & & & Luminaires/D & $\begin{array}{l}\text { Luminaire efficiency looks } \\
\text { wrong. The DL and WW } \\
\text { have the exact same } \\
\text { efficiency? }\end{array}$ & Content & 4 & 1 & 4 & 3.4 & 4 & 4 & 5 & 4.3 & 3.8 & 3.5 \\
\hline
\end{tabular}


Appendix E: Master Comments List

\begin{tabular}{|c|c|c|c|c|c|c|c|c|c|c|c|c|c|c|c|c|c|c|c|c|}
\hline $\begin{array}{l}\text { Commenter } \\
\text { ID \# }\end{array}$ & $\begin{array}{l}\text { Comment } \\
\text { Reference } \\
\#\end{array}$ & $\begin{array}{l}\text { Commenter } \\
\text { Category }\end{array}$ & \begin{tabular}{|l} 
Comment \\
Type
\end{tabular} & Space Type & \begin{tabular}{|l|} 
Ceiling \\
Height/ \\
Total Area
\end{tabular} & \begin{tabular}{|l}
$\#$ \\
workstations/ \\
partition \\
height (open \\
office only)
\end{tabular} & $\begin{array}{l}\text { Daylight } \\
\text { Input (if } \\
\text { applicable) }\end{array}$ & \begin{tabular}{|l|} 
Reference webpage \\
or other location \\
(see \\
"Reference.doc") \\
\end{tabular} & $\begin{array}{l}\text { Comment Description: } \\
\text { Issues, Suggestions \& } \\
\text { Requests }\end{array}$ & Action/Solutio & \begin{tabular}{|l|} 
St Status \\
or \\
Ticket \#
\end{tabular} & $\begin{array}{c}\text { Frequenc } \\
\text { y (20\%) }\end{array}$ & $\begin{array}{c}\text { Importance } \\
\text { of comment } \\
(80 \%)\end{array}$ & $\begin{array}{c}\text { Sum } \\
(100 \%)\end{array}$ & $\begin{array}{c}\text { Ease of } \\
\text { Implementatio } \\
\mathrm{n}(30 \%)\end{array}$ & $\begin{array}{c}\text { Duration } \\
(40 \%)\end{array}$ & $\begin{array}{c}\text { Risk/ } \\
\text { Complicatio } \\
\mathrm{n}(30 \%)\end{array}$ & \begin{tabular}{|c} 
Sum \\
$(100 \%)$
\end{tabular} & $\begin{array}{c}\text { Short Term } \\
\text { (40\% } \\
\text { Importance) } \\
\text { (60\% Feasibility) }\end{array}$ & $\begin{array}{c}\text { Long Term } \\
\text { (85\% } \\
\text { Importance) } \\
\text { (15\% Feasibility) }\end{array}$ \\
\hline N Miller & NM9.3 & Internal & & & & & & & $\begin{array}{l}\text { How are luminaires A and B } \\
\text { different? I can't see the } \\
\text { photometric distribution, } \\
\text { and the descriptions look } \\
\text { the same. }\end{array}$ & 3 & 4 & 1 & 4 & 3.4 & 4 & 4 & 5 & 4.3 & 3.8 & 3.5 \\
\hline M Myer & MM464 & Internal & \begin{tabular}{|l|} 
Appearance \\
Aesthetics
\end{tabular} & \begin{tabular}{|l|} 
Recessed Non- \\
Planar on wider \\
centers
\end{tabular} & $9.6 / 5000$ & $40 / 48$ & & Luminaires $\mathrm{B}$ & $\begin{array}{l}\text { Why is the power suppy } \\
\text { remotely located? }\end{array}$ & Content & 4 & 2 & 4 & 3.6 & 4 & 4 & 5 & 4.3 & 3.9 & 3.7 \\
\hline \begin{tabular}{|l|} 
A Cortese \\
\end{tabular} & AC16 & Architect & Content & & & & & & \begin{tabular}{|l|} 
Conference room vignettes \\
- do all of these really have \\
the same LPD of 0.77 \\
W/SF? That seems \\
unlikely.
\end{tabular} & Content & 2 & 2 & 5 & 4.4 & 3 & 3 & 5 & 3.6 & 4.1 & 4.3 \\
\hline \begin{tabular}{|l} 
M Dare \\
\end{tabular} & MD7 & Utility & & & $\begin{array}{l}10 / 4000 \\
S F\end{array}$ & $\begin{array}{l}70 \\
\text { workstations, } \\
60 \text { : partition } \\
\text { height }\end{array}$ & \begin{tabular}{|l|}
1080 feet \\
length, \\
sidelight \\
glazing \\
area is \\
4320.
\end{tabular} & \begin{tabular}{|l} 
Recessed non- \\
planar lensed \\
luminaires. Open \\
plan office w/low \\
ceiling and low \\
partitions.
\end{tabular} & $\begin{array}{l}\text { Why does a 60" partition } \\
\text { height yield ONLY the } \\
\text { recessed non-planar } \\
\text { lensed luminaires, which } \\
\text { indicates that it's for low } \\
\text { partitions? }\end{array}$ & Content & 2 & 2 & 5 & 4.4 & 4 & 3 & 4 & 3.6 & 4.1 & 4.3 \\
\hline M Myer & MM202 & Internal & \begin{tabular}{|l|} 
Content \\
\end{tabular} & \begin{tabular}{|l} 
Add Corridors \\
Space/5' wide
\end{tabular} & & & corridors & & \begin{tabular}{|l|}
4 vignettes are shown here. \\
It looks like they are \\
duplicative. I see (2) \\
Recessed 4 ' asymmetrical \\
wall-wash luminaires and \\
(2) recessed 1'x4' non- \\
plananr lensed luminaires \\
options shown. Is this \\
right? If so, why are all fo \\
the LPDs the same?
\end{tabular} & Content & 2 & 2 & 5 & 4.4 & 4 & 3 & 4 & 3.6 & 4.1 & 4.3 \\
\hline N Ferzacca & NF2 & Engineer & & & & & & & $\begin{array}{l}\text { It didn't give me any } \\
\text { vignettes when I 1st put in } \\
\text { my choices. Said that there } \\
\text { were no vignettes for my } \\
\text { project, why is that? }\end{array}$ & Content & 2 & 2 & 5 & 4.4 & 4 & 3 & 4 & 3.6 & 4.1 & 4.3 \\
\hline N Ferzacca & NF6 & Engineer & & & \begin{tabular}{|l|}
10 \\
ft/10,000 \\
SF
\end{tabular} & $\begin{array}{l}75 \\
\text { workstations, } \\
48 "\end{array}$ & $\mathrm{~N} / \mathrm{A}$ & Non-planar lensed. & $\begin{array}{l}\text { Why is there only one } \\
\text { vignette? }\end{array}$ & Content & 5 & 2 & 5 & 4.4 & 4 & 3 & 4 & 3.6 & 4.1 & 4.3 \\
\hline J Yorgey & JY2 & Manufacturer & $\mid$ Functionality & & & & & \begin{tabular}{|l|} 
Project description \\
energy estimate
\end{tabular} & \begin{tabular}{|l|} 
selected Pennsylvania \\
code type did not change - \\
should it? Also ASHRAE is \\
listed as "codes" it is a \\
standard not a code. \\
Pennsylvania uses \\
IECC2009 it is not on the \\
list.
\end{tabular} & Content & 7 & 2 & 5 & 4.4 & 3 & 3 & 5 & 3.6 & 4.1 & 4.3 \\
\hline \begin{tabular}{|l|l} 
L Davis \\
\end{tabular} & LD4 & Designer & & & & & & & I need the Title 24 baseline! & Content & 7 & 2 & 5 & 4.4 & 3 & 3 & 5 & 3.6 & 4.1 & 4.3 \\
\hline
\end{tabular}


Appendix E: Master Comments List

\begin{tabular}{|c|c|c|c|c|c|c|c|c|c|c|c|c|c|c|c|c|c|c|c|c|}
\hline $\begin{array}{l}\text { Commenter } \\
\text { ID \# }\end{array}$ & $\begin{array}{l}\text { Comment } \\
\text { Reference } \\
\#\end{array}$ & $\begin{array}{l}\text { Commenter } \\
\text { Category }\end{array}$ & $\begin{array}{l}\text { Comment } \\
\text { Type }\end{array}$ & Space Type & \begin{tabular}{|l|} 
Ceiling \\
Height/ \\
Total Area
\end{tabular} & \begin{tabular}{|l}
$\#$ \\
workstations/ \\
partition \\
height (open \\
office only)
\end{tabular} & \begin{tabular}{|l} 
Daylight \\
Input (if \\
applicable)
\end{tabular} & \begin{tabular}{|l|} 
Reference webpage \\
or other location \\
(see \\
"Reference.doc") \\
\end{tabular} & $\begin{array}{l}\text { Comment Description: } \\
\text { Issues, Suggestions \& } \\
\text { Requests }\end{array}$ & Action/Solution & \begin{tabular}{|l|} 
Status \\
or \\
Ticket \#
\end{tabular} & $\begin{array}{l}\text { Frequenc } \\
\mathrm{y}(20 \%)\end{array}$ & $\begin{array}{c}\text { Importance } \\
\text { of comment } \\
(80 \%)\end{array}$ & $\begin{array}{c}\text { Sum } \\
(100 \%)\end{array}$ & $\begin{array}{c}\text { Ease of } \\
\text { Implementatio } \\
\mathrm{n}(30 \%)\end{array}$ & $\begin{array}{c}\text { Duration } \\
(40 \%)\end{array}$ & $\begin{array}{c}\text { Risk/ } \\
\text { Complicatio } \\
\mathrm{n}(30 \%)\end{array}$ & \begin{tabular}{|} 
Sum \\
$(100 \%)$
\end{tabular} & \begin{tabular}{|c|} 
Short Term \\
$(40 \%$ \\
Importance) \\
(60\% Feasibility)
\end{tabular} & $\begin{array}{c}\text { Long Term } \\
(85 \% \\
\text { Importance) } \\
\text { (15\% Feasibility) }\end{array}$ \\
\hline A Cortese & AC21 & Architect & Functionality & & & & & & \begin{tabular}{|l|} 
Private Office Control \\
Strategies are not named \\
like the control strategies in \\
other types of spaces. And \\
if I choose one, it doesn't \\
show up in the summary of \\
my space. Only "no \\
controls other than required \\
by code" shows up for \\
private offices.
\end{tabular} & Content & 11 & 3 & 5 & 4.6 & 4 & 3 & 4 & 3.6 & 4.2 & 4.5 \\
\hline \begin{tabular}{|l} 
D Maniccia \\
\end{tabular} & DM6 & Controls & & & & & & \begin{tabular}{|l|} 
Suspended D/I \\
luminaires with low \\
BF ballasts
\end{tabular} & \begin{tabular}{|l|} 
You wouldn't need 3 \\
luminaires in a 150SF \\
office. Either the design is \\
wrong, or you are offering \\
vignettes that aren't \\
appropriate for the office \\
size I entered.
\end{tabular} & Content & 2 & 1 & 5 & 4.2 & 4 & 4 & 5 & 4.3 & 4.2 & 4.2 \\
\hline A Cortese & AC12 & Architect & Content & & & & & & $\begin{array}{l}\text { All of the ' are actually ? on } \\
\text { my computer's browser. }\end{array}$ & Content & 6 & 5 & 4 & 4.2 & 4 & 4 & 5 & 4.3 & 4.2 & 4.2 \\
\hline HMcKay & HM9 & Designer & Content & & & & & & $\begin{array}{l}\text { Symbols for inches and } \\
\text { dashes are sometimes } \\
\text { shown as question marks. }\end{array}$ & Content & 6 & 5 & 4 & 4.2 & 4 & 4 & 5 & 4.3 & 4.2 & 4.2 \\
\hline \begin{tabular}{|l|} 
JBeutell \\
\end{tabular} & JB4 & Owner & & & & & & & typos. & Content & 6 & 5 & 4 & 4.2 & 4 & 4 & 5 & 4.3 & 4.2 & 4.2 \\
\hline |J Yorgey & JY10 & Manufacturer & Functionality & & & & & \begin{tabular}{|l|} 
Control stratigies, \\
daylight occ sense
\end{tabular} & $\begin{array}{l}\text { ? Mark in "they're" This } \\
\text { one says dimming for } \\
\text { daylight control - sho }\end{array}$ & Content & 6 & 5 & 4 & 4.2 & 4 & 4 & 5 & 4.3 & 4.2 & 4.2 \\
\hline |J Yorgey & JY16 & Manufacturer & Functionality & & & & & & $\begin{array}{l}\text { how should Ft be inputed } \\
\text { with or qith out marks - ' " ? }\end{array}$ & Content & 6 & 5 & 4 & 4.2 & 4 & 4 & 5 & 4.3 & 4.2 & 4.2 \\
\hline K Abernathy & KA9 & Designer & General & Pharmacy & & & & \begin{tabular}{|l|} 
Control Details - \\
Occupancy Sensors
\end{tabular} & $\begin{array}{l}\text { Check the fonts because I } \\
\text { have question marks } \\
\text { around the words see, hear } \\
\text { and seeing. Also the } \\
\text { spacing seems to be funky } \\
\text { in some of these. Could be } \\
\text { my web browser not sure. }\end{array}$ & Content & 6 & 5 & 4 & 4.2 & 4 & 4 & 5 & 4.3 & 4.2 & 4.2 \\
\hline LDavis & LD8 & Designer & & & & & & & \begin{tabular}{|l|} 
Text corrections are \\
needed -- "?" marks are \\
showing up where you \\
probably mean to use \\
dashes
\end{tabular} & Content & 6 & 5 & 4 & 4.2 & 4 & 4 & 5 & 4.3 & 4.2 & 4.2 \\
\hline M Dare & MD11 & Utility & & & & & & & \begin{tabular}{|l|} 
Typos. \\
\end{tabular} & Content & 6 & 5 & 4 & 4.2 & 4 & 4 & 5 & 4.3 & 4.2 & 4.2 \\
\hline M Myer & MM66 & Internal & Content & \begin{tabular}{|l|} 
Recessed Slot \\
Lumianirs \\
parallel to desk \\
with compact \\
fluorescent wall- \\
washers (office)
\end{tabular} & & & & Luminaire B & $\begin{array}{l}\text { Text issue in the inch tick } \\
\text { mark and is being shown } \\
\text { as quotes }\end{array}$ & Content & 6 & 5 & 4 & 4.2 & 4 & 4 & 5 & 4.3 & 4.2 & 4.2 \\
\hline N Miller & NM12 & Internal & Content & Office-corridor & & & & \begin{tabular}{|l|} 
recessed asymm \\
wallwash lum's
\end{tabular} & \begin{tabular}{|l|} 
Replace "?" with "' ' " in \\
three places under \\
concept. \\
Replace "?" in Strategy box \\
as well.
\end{tabular} & Content & 6 & 5 & 4 & 4.2 & 4 & 4 & 5 & 4.3 & 4.2 & 4.2 \\
\hline W Dau & WD5 & \begin{tabular}{|l|}
$\begin{array}{l}\text { Designer/IES } \\
\text { Chair }\end{array}$ \\
\end{tabular} & & & & & & & $\begin{array}{l}\text { "?" showing up instead of } \\
\text { an apostrophe. }\end{array}$ & Content & 6 & 5 & 4 & 4.2 & 4 & 4 & 5 & 4.3 & 4.2 & 4.2 \\
\hline
\end{tabular}


Appendix E: Master Comments List

\begin{tabular}{|c|c|c|c|c|c|c|c|c|c|c|c|c|c|c|c|c|c|c|c|c|}
\hline $\begin{array}{l}\text { Commenter } \\
\text { ID \# }\end{array}$ & $\begin{array}{l}\text { Comment } \\
\text { Reference } \\
\#\end{array}$ & $\begin{array}{l}\text { Commenter } \\
\text { Category }\end{array}$ & \begin{tabular}{|l} 
Comment \\
Type
\end{tabular} & Space Type & \begin{tabular}{|l|} 
Ceiling \\
Height/ \\
Total Area
\end{tabular} & \begin{tabular}{|l|}
$\#$ \\
workstations/ \\
partition \\
height (open \\
office only) \\
\end{tabular} & \begin{tabular}{|l|} 
Daylight \\
Input (if \\
applicable)
\end{tabular} & \begin{tabular}{|l|} 
Reference webpage \\
or other location \\
(see \\
"Reference.doc") \\
\end{tabular} & \begin{tabular}{|l|} 
Comment Description: \\
Issues, Suggestions \& \\
Requests
\end{tabular} & Action/Solu & \begin{tabular}{|l|} 
Status \\
or \\
Ticket \#
\end{tabular} & $\begin{array}{c}\text { Frequenc } \\
\mathrm{y}(20 \%)\end{array}$ & $\begin{array}{c}\text { Importance } \\
\text { of comment } \\
(80 \%)\end{array}$ & \begin{tabular}{|c|} 
Sum \\
$(100 \%)$
\end{tabular} & $\begin{array}{c}\text { Ease of } \\
\text { Implementatio } \\
\mathrm{n}(30 \%)\end{array}$ & $\begin{array}{c}\text { Duration } \\
(40 \%)\end{array}$ & $\begin{array}{c}\text { Risk/ } \\
\text { Complicatio } \\
\mathrm{n}(30 \%)\end{array}$ & \begin{tabular}{|} 
Sum \\
$(100 \%)$
\end{tabular} & \begin{tabular}{|c|} 
Short Term \\
$(40 \%$ \\
Importance) \\
(60\% Feasibility)
\end{tabular} & $\begin{array}{c}\text { Long Term } \\
\text { (85\% } \\
\text { Importance) } \\
\text { (15\% Feasibility) }\end{array}$ \\
\hline C DiLouie & CD3 & Core Team & Content & & & & & & \begin{tabular}{|l|} 
When I get to the reception \\
area section, I am offered \\
several lighting \\
design templates. For one \\
of them, a control \\
templates is not available: \\
"recessed CFL downlights \\
with dropped decorative \\
diffuser and perimeter wall \\
slots." We will need a \\
control template for this \\
lighting template.
\end{tabular} & Content & 11 & 2 & 5 & 4.4 & 3 & 4 & 5 & 4 & 4.2 & 4.3 \\
\hline M Myer & \begin{tabular}{|l|l|} 
MM387 \\
\end{tabular} & Internal & Content & \begin{tabular}{|l|} 
Add Open Plan \\
Space
\end{tabular} & $9.6 / 5000$ & 40/48 & & LPDs & \begin{tabular}{|l|} 
These values all look low. I \\
am surprised. If accurate, \\
congratulations.
\end{tabular} & Content & 2 & 2 & 5 & 4.4 & 4 & 4 & 5 & 4.3 & 4.4 & 4.4 \\
\hline M Myer & \begin{tabular}{|l|} 
MM37 \\
\end{tabular} & Internal & General & \begin{tabular}{|l|} 
Suspended \\
Direct/lndirect \\
Luminaires with \\
a Whiteboard \\
Light
\end{tabular} & $\begin{array}{l}\text { Conferenc } \\
\text { e room }\end{array}$ & & & General & $\begin{array}{l}\text { What is the difference } \\
\text { between this vignette and } \\
\text { the other vignette. I cannot } \\
\text { find any major differences } \\
\text { other than the LPD and I } \\
\text { cannot figure out why the } \\
\text { LPD is different. }\end{array}$ & Content & 2 & 2 & 5 & 4.4 & 4 & 4 & 5 & 4.3 & 4.4 & 4.4 \\
\hline M Myer & MM606 & Internal & Content & \begin{tabular}{|l|} 
Perimeter \\
Recessed \\
Linear \\
Fluorescent
\end{tabular} & & & & LPD & $\begin{array}{l}\text { Should the the } \\
\text { power/energy unit be in } \\
\text { W/sf or should it be W/f?? }\end{array}$ & Content & 2 & 2 & 5 & 4.4 & 4 & 4 & 5 & 4.3 & 4.4 & 4.4 \\
\hline M Myer & \begin{tabular}{|l|l|} 
MM417 \\
\end{tabular} & Internal & Content & \begin{tabular}{|l|}
$\begin{array}{l}\text { Suspended 12' } \\
\text { single-lamp }\end{array}$ \\
\end{tabular} & $9.6 / 5000$ & $40 / 48$ & & Luminaires A & $\begin{array}{l}\text { Why are we separating } \\
\text { these as two luminaires } \\
\text { when the title of the } \\
\text { vigentte is } 12 \text { ? }\end{array}$ & Content & 4 & 2 & 5 & 4.4 & 4 & 4 & 5 & 4.3 & 4.4 & 4.4 \\
\hline M Myer & MM632 & Internal & Content & \begin{tabular}{|l|} 
Add Open Plan \\
Space- \\
Daylighting \\
questions \\
\end{tabular} & & & & & $\begin{array}{l}\text { This section NEEDS more } \\
\text { FAQs about all of the } \\
\text { different options that we are } \\
\text { asking about. }\end{array}$ & Content & 46 & 2 & 5 & 4.4 & 4 & 4 & 5 & 4.3 & 4.4 & 4.4 \\
\hline K Cota & KC5 & Utility & & & & & & \begin{tabular}{|l|} 
Kelly Cota, National \\
Grid
\end{tabular} & $\begin{array}{l}\text { Uh oh, manufacturer- } \\
\text { specific codes are in the } \\
\text { schedule and Energy } \\
\text { Estimate report. }\end{array}$ & Content & 3 & 1 & 5 & 4.2 & 4 & 5 & 5 & 4.7 & 4.4 & 4.3 \\
\hline HMcKay & HM3 & Designer & Content & & & & & & $\begin{array}{l}\text { Needs definitions either as } \\
\text { links or mouse-overs. Eg., } \\
\text { vacancy sensors, MLPW, } \\
\text { etc. }\end{array}$ & Content & 1 & 4 & 5 & 4.8 & 4 & 3 & 5 & 3.9 & 4.4 & 4.7 \\
\hline
\end{tabular}




\begin{tabular}{|c|c|c|c|c|c|c|c|c|c|c|c|c|c|c|c|c|c|c|c|c|}
\hline $\begin{array}{l}\text { Commenter } \\
\text { ID \# }\end{array}$ & \begin{tabular}{|l|} 
Comment \\
Reference \\
$\#$
\end{tabular} & $\begin{array}{l}\text { Commenter } \\
\text { Category }\end{array}$ & \begin{tabular}{|l} 
Comment \\
Type
\end{tabular} & Space Type & \begin{tabular}{|l|} 
Ceiling \\
Height/ \\
Total Area
\end{tabular} & \begin{tabular}{|l}
$\#$ \\
workstations/ \\
partition \\
height (open \\
office only) \\
\end{tabular} & $\mid \begin{array}{l}\text { Daylight } \\
\text { Input (if } \\
\text { applicable) }\end{array}$ & \begin{tabular}{|l|} 
Reference webpage \\
or other location \\
(see \\
"Reference.doc")
\end{tabular} & $\begin{array}{l}\text { Comment Description: } \\
\text { Issues, Suggestions \& } \\
\text { Requests }\end{array}$ & Action/Solution & \begin{tabular}{|l|}
$\begin{array}{l}\text { Status } \\
\text { or } \\
\text { Ticket \# }\end{array}$ \\
\end{tabular} & $\begin{array}{c}\text { Frequenc } \\
\mathrm{y}(20 \%)\end{array}$ & $\begin{array}{c}\text { Importance } \\
\text { of comment } \\
(80 \%)\end{array}$ & \begin{tabular}{|l|} 
Sum \\
$(100 \%)$
\end{tabular} & $\begin{array}{c}\text { Ease of } \\
\text { Implementatio } \\
\mathrm{n}(30 \%)\end{array}$ & \begin{tabular}{|c|} 
Duration \\
$(40 \%)$
\end{tabular} & $\begin{array}{c}\text { Risk/ } \\
\text { Complicatio } \\
\mathrm{n}(30 \%)\end{array}$ & \begin{tabular}{|} 
Sum \\
$(100 \%)$
\end{tabular} & $\begin{array}{c}\text { Short Term } \\
(40 \% \\
\text { Importance) } \\
(60 \% \text { Feasibility) } \\
\end{array}$ & $\begin{array}{c}\text { Long Term } \\
(85 \% \\
\text { Importance) } \\
\text { (15\% Feasibility) }\end{array}$ \\
\hline HMcKay & HM13 & Designer & Content & & & & & & \begin{tabular}{|l|} 
\\
VT - Spell out "Visual Light \\
Transmission VLT"
\end{tabular} & Content & 1 & 4 & 5 & 4.8 & 4 & 3 & 5 & 3.9 & 4.4 & 4.7 \\
\hline D Maniccia & DM11 & Controls & . & & & & & Strategy tab & \begin{tabular}{|l|} 
Definition of vacancy \\
sensor is manual on, auto \\
off, but it's implicit in the \\
definition that it's a wall \\
switch. Is an vacancy \\
sensor, by definition, also a \\
wall switch? Check the info \\
I sent to Carol from the T24 \\
code. Section 119, page \\
69.
\end{tabular} & Content & 1 & 3 & 5 & 4.6 & 4 & 4 & 5 & 4.3 & 4.5 & 4.6 \\
\hline HMcKay & HM19 & Designer & Content & & & & & & $\begin{array}{l}\text { Vacancy sensors and other } \\
\text { terms need to be defined. }\end{array}$ & Content & 1 & 3 & 5 & 4.6 & 4 & 4 & 5 & 4.3 & 4.5 & 4.6 \\
\hline \begin{tabular}{|l|l|} 
LDavis \\
\end{tabular} & $\mid$ LD13 & Designer & & & & & & & \begin{tabular}{|l|} 
The state code in CA \\
doesn't use the term \\
vacancy sensor, they say \\
occupancy sensor, manual \\
on. Not everyone will know \\
what you mean by vacancy \\
sensor. Is there a way to \\
underline these new-ish \\
terms, and hyperlink to a \\
definition?
\end{tabular} & Content & 1 & 3 & 5 & 4.6 & 4 & 4 & 5 & 4.3 & 4.5 & 4.6 \\
\hline K Cota & KC2 & Utility & & & & & & $\begin{array}{l}\text { Kelly Cota, National } \\
\text { Grid }\end{array}$ & $\begin{array}{l}\text { explanation of what } \\
\text { baseline means, should be } \\
\text { in the tool }\end{array}$ & Content & 15 & 3 & 5 & 4.6 & 4 & 4 & 5 & 4.3 & 4.5 & 4.6 \\
\hline E Smith & ES1 & Owner & & & & & & & What are light shelves? & Content & 1 & 1 & 5 & 4.2 & 5 & 5 & 5 & 5 & 4.5 & 4.3 \\
\hline N Ferzacca & NF9 & Engineer & & & & & & & $\begin{array}{l}\text { How are you going to share } \\
\text { the layout strategies with } \\
\text { people? Do you want } \\
\text { them to come away with } \\
\text { spacing info? }\end{array}$ & Content & 9 & 1 & 5 & 4.2 & 5 & 5 & 5 & 5 & 4.5 & 4.3 \\
\hline \begin{tabular}{|l|} 
D Maniccia \\
\end{tabular} & DM12 & \begin{tabular}{|l|} 
Controls \\
\end{tabular} & & & & & & & $\begin{array}{l}\text { Is there a way to evaluate } \\
\text { the language that is written } \\
\text { for controls, without trying } \\
\text { to go through every } \\
\text { combination of templates? }\end{array}$ & Content & 12 & 1 & 5 & 4.2 & 5 & 5 & 5 & 5 & 4.5 & 4.3 \\
\hline
\end{tabular}


Appendix E: Master Comments List

\begin{tabular}{|c|c|c|c|c|c|c|c|c|c|c|c|c|c|c|c|c|c|c|c|c|}
\hline $\begin{array}{l}\text { Commenter } \\
\text { ID \# }\end{array}$ & $\begin{array}{l}\text { Comment } \\
\text { Reference } \\
\#\end{array}$ & \begin{tabular}{|l} 
Commenter \\
Category
\end{tabular} & \begin{tabular}{|l} 
Comment \\
Type
\end{tabular} & Space Type & \begin{tabular}{|l|} 
Ceiling \\
Height/ \\
Total Area
\end{tabular} & \begin{tabular}{|l} 
\\
workstations/ \\
partition \\
height (open \\
office only) \\
\end{tabular} & $\left|\begin{array}{l}\text { Daylight } \\
\text { Input (if } \\
\text { applicable) }\end{array}\right|$ & \begin{tabular}{|l|} 
Reference webpage \\
or other location \\
(see \\
"Reference.doc")
\end{tabular} & $\begin{array}{l}\text { Comment Description: } \\
\text { Issues, Suggestions \& } \\
\text { Requests }\end{array}$ & Action/Sol & \begin{tabular}{|l|} 
Status \\
or \\
Ticket \#
\end{tabular} & $\begin{array}{c}\text { Frequenc } \\
\text { y (20\%) }\end{array}$ & $\begin{array}{c}\text { Importance } \\
\text { of comment } \\
(80 \%)\end{array}$ & \begin{tabular}{|c|} 
Sum \\
$(100 \%)$
\end{tabular} & $\begin{array}{c}\text { Ease of } \\
\text { Implementatio } \\
\mathrm{n}(30 \%)\end{array}$ & $\begin{array}{c}\text { Duration } \\
(40 \%)\end{array}$ & $\begin{array}{c}\text { Risk/ } \\
\text { Complicatio } \\
\mathrm{n}(30 \%)\end{array}$ & \begin{tabular}{|c|} 
Sum \\
$(100 \%)$
\end{tabular} & $\begin{array}{c}\text { Short Term } \\
(40 \% \\
\text { Importance) } \\
(60 \% \text { Feasibility) }\end{array}$ & $\begin{array}{c}\text { Long Term } \\
(85 \% \\
\text { Importance) } \\
\text { (15\% Feasibility) }\end{array}$ \\
\hline M Myer & MM13 & Internal & Content & \begin{tabular}{|l|} 
Recessed Non- \\
Planar Lensed \\
Luminaires with \\
low Ballast \\
Factor and \\
Recessed \\
Compact \\
Fluorescent \\
Lensed Wall- \\
washers \\
(Office) \\
\end{tabular} & \begin{tabular}{|l|} 
Conferenc \\
e room
\end{tabular} & & & \begin{tabular}{|l|} 
Strategy/Luminaire \\
A
\end{tabular} & \begin{tabular}{|l|} 
am troubled with this \\
statement "These \\
luminaires are significantly \\
more efficienct than those \\
with open-celled parabolic \\
loubers, so they reduce \\
energy consumption and \\
the quantity of luminaires in \\
the space". Do we really \\
think less fixtures were \\
installed? \\
\end{tabular} & Content & 4 & 2 & 5 & 4.4 & 4 & 5 & 5 & 4.7 & 4.5 & 4.4 \\
\hline \begin{tabular}{|l|} 
A Cortese \\
\end{tabular} & AC6 & Architect & Content & & & & & & \begin{tabular}{|l|} 
I would suggest you try and \\
eliminate to the use of \\
acronyms (VT, BF, LPD, \\
AFF) wherever possible. \\
Direct hyperlinks to \\
definitions might provide \\
added functionality. \\
\end{tabular} & Content & 1 & 4 & 5 & 4.8 & 4 & 4 & 5 & 4.3 & 4.6 & 4.7 \\
\hline C DiLouie & \begin{tabular}{|l|} 
CD8 \\
\end{tabular} & Core Team & Content & & & & & & $\begin{array}{l}\text { That box asked me for "VT } \\
\text { of sidelighting"-the term } \\
\text { "VT" should be spelled out } \\
\text { at least, at most explained }\end{array}$ & Content & 1 & 4 & 5 & 4.8 & 4 & 4 & 5 & 4.3 & 4.6 & 4.7 \\
\hline \begin{tabular}{|l|} 
C DiLouie \\
\end{tabular} & CD9 & Core Team & Content & & & & & & $\begin{array}{l}\text { Lighting options showed } \\
\text { me "low BF" ballasts-the } \\
\text { term "BF" should be spelled } \\
\text { out at least, at most } \\
\text { explained }\end{array}$ & Content & 1 & 4 & 5 & 4.8 & 4 & 4 & 5 & 4.3 & 4.6 & 4.7 \\
\hline \begin{tabular}{|l|}
$J$ Beutell \\
\end{tabular} & JB1 & Owner & & & & & & & $\begin{array}{l}\text { What is sidelighting glazing } \\
\text { in open office? How about } \\
\text { using the word windows? } \\
\text { Curtain wall, window wall, } \\
\text { saying the same thing with } \\
3 \text { different words. How } \\
\text { about a glossery. }\end{array}$ & Content & 1 & 4 & 5 & 4.8 & 4 & 4 & 5 & 4.3 & 4.6 & 4.7 \\
\hline \begin{tabular}{|l} 
J Beutell \\
\end{tabular} & JB2 & Owner & & & & & & & What is VT? (spell it out) & Content & 1 & 4 & 5 & 4.8 & 4 & 4 & 5 & 4.3 & 4.6 & 4.7 \\
\hline J Yorgey & JY7 & Manufacturer & Content & & & & & \begin{tabular}{|l|} 
Space type \\
summary open plan
\end{tabular} & $\begin{array}{l}\text { Don't know what is meant } \\
\text { by Sidelight glazing area or } \\
\text { VT type }\end{array}$ & Content & 1 & 4 & 5 & 4.8 & 4 & 4 & 5 & 4.3 & 4.6 & 4.7 \\
\hline M Myer & MM656 & Internal & Content & \begin{tabular}{|l|} 
Controls \\
Strategies: \\
Vacancy \\
Sensors, \\
Daylight \\
Harvesting \\
\end{tabular} & & & & Strategy & $\begin{array}{l}\text { 5th bullet - I think that you } \\
\text { need to define "head height } \\
\text { of the window" }\end{array}$ & Content & 1 & 4 & 5 & 4.8 & 4 & 4 & 5 & 4.3 & 4.6 & 4.7 \\
\hline N Ferzacca & NF4 & Engineer & & & & & & & $\begin{array}{l}\text { Should we be asking for VT } \\
\text { or VLT? Should be spelling } \\
\text { out the acronym. }\end{array}$ & Content & 1 & 4 & 5 & 4.8 & 4 & 4 & 5 & 4.3 & 4.6 & 4.7 \\
\hline
\end{tabular}




\begin{tabular}{|c|c|c|c|c|c|c|c|c|c|c|c|c|c|c|c|c|c|c|c|c|}
\hline $\begin{array}{l}\text { Commenter } \\
\text { ID \# }\end{array}$ & $\begin{array}{l}\text { Comment } \\
\text { Reference } \\
\#\end{array}$ & $\begin{array}{l}\text { Commenter } \\
\text { Category }\end{array}$ & \begin{tabular}{|l} 
Comment \\
Type
\end{tabular} & Space Type & \begin{tabular}{|l|} 
Ceiling \\
Height// \\
Total Area
\end{tabular} & \begin{tabular}{|l}
$\#$ \\
workstations/ \\
partition \\
height (open \\
office only) \\
\end{tabular} & $\mid \begin{array}{l}\text { Daylight } \\
\text { Input (if } \\
\text { applicable) }\end{array}$ & \begin{tabular}{|l|} 
Reference webpage \\
or other location \\
(see \\
"Reference.doc") \\
\end{tabular} & $\begin{array}{l}\text { Comment Description: } \\
\text { Issues, Suggestions \& } \\
\text { Requests }\end{array}$ & Action/Solution & \begin{tabular}{|l|} 
Status \\
or \\
Ticket \#
\end{tabular} & $\begin{array}{c}\text { Frequenc } \\
\mathrm{y}(20 \%)\end{array}$ & $\begin{array}{c}\text { Importance } \\
\text { of comment } \\
(80 \%)\end{array}$ & \begin{tabular}{|c|} 
Sum \\
$(100 \%)$
\end{tabular} & $\begin{array}{c}\text { Ease of } \\
\text { Implementatio } \\
\mathrm{n}(30 \%)\end{array}$ & $\begin{array}{c}\text { Duration } \\
(40 \%)\end{array}$ & $\begin{array}{c}\text { Risk/ } \\
\text { Complicatio } \\
\mathrm{n}(30 \%)\end{array}$ & \begin{tabular}{|c|} 
Sum \\
$(100 \%)$
\end{tabular} & $\begin{array}{c}\text { Short Term } \\
(40 \% \\
\text { Importance) } \\
(60 \% \text { Feasibility) } \\
\end{array}$ & $\begin{array}{c}\text { Long Term } \\
(85 \% \\
\text { Importance) } \\
\text { (15\% Feasibility) }\end{array}$ \\
\hline LDavis & 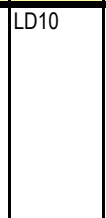 & Designer & & & & & & & $\begin{array}{l}\text { The term "non-planar" is } \\
\text { not often used, but I don't } \\
\text { know what term is better. } \\
\text { I'm hearing "volumetric" } \\
\text { style a lot. Maybe put } \\
\text { "volumetric" in parentheses } \\
\text { next to non-planar. }\end{array}$ & Content & 3 & 4 & 5 & 4.8 & 4 & 4 & 5 & 4.3 & 4.6 & 4.7 \\
\hline M Dare & MD8 & Utility & & & & & & & \begin{tabular}{|l|} 
What exactly do you mean \\
by non-planar? Perhaps \\
you should call it \\
"volumetric" type \\
luminaires.
\end{tabular} & Content & 3 & 4 & 5 & 4.8 & 4 & 4 & 5 & 4.3 & 4.6 & 4.7 \\
\hline M Myer & MM349 & Internal & Content & \begin{tabular}{|l|} 
Suspended \\
Direct/Indirect \\
Luminaires with \\
low BF ballasts
\end{tabular} & $9^{\prime}$ & PO & & $\begin{array}{l}\text { Strategy/Luminaires } \\
\text { A }\end{array}$ & $\begin{array}{l}\text { Change "bi-directional" to } \\
\text { "indirect/direct" }\end{array}$ & Content & 3 & 4 & 5 & 4.8 & 4 & 4 & 5 & 4.3 & 4.6 & 4.7 \\
\hline N Ferzacca & NF22 & Engineer & & & & & & & $\begin{array}{l}\text { Non-planar. Premium } \\
\text { recessed indirect. NStar } \\
\text { uses Volumetric. }\end{array}$ & Content & 3 & 4 & 5 & 4.8 & 4 & 4 & 5 & 4.3 & 4.6 & 4.7 \\
\hline C DiLouie & CD30 & Core Team & Content & & & & & & $\begin{array}{l}\text { The control options all have } \\
\text { weird numbering in the } \\
\text { vignette documents, such } \\
\text { as "control system: } 41-9 . " \\
\text { This needs to be cleaned } \\
\text { up. }\end{array}$ & Content & 14 & 3 & 5 & 4.6 & 5 & 4 & 5 & 4.6 & 4.6 & 4.6 \\
\hline \begin{tabular}{|l|} 
C DiLouie \\
\end{tabular} & CD4 & Core Team & Content & & & & & & \begin{tabular}{|l|} 
I selected another \\
reception area section \\
lighting template and \\
picked the \\
control template available. \\
The section diagram has \\
two typos
\end{tabular} & Content & 13 & 3 & 5 & 4.6 & 5 & 5 & 5 & 5 & 4.8 & 4.7 \\
\hline M Myer & MM643 & Internal & Content & \begin{tabular}{|l|} 
Controls \\
Strategies: \\
Scheduling, \\
Daylight \\
Harvesting \\
\end{tabular} & & & & Controls & $\begin{array}{l}\text { I think that you need a } \\
\text { better title for this section }\end{array}$ & \begin{tabular}{|l|} 
Controls \\
Templates
\end{tabular} & 14 & 1 & 2 & 1.8 & 5 & 5 & 5 & 5 & 3.1 & 2.3 \\
\hline W Dau & WD6 & \begin{tabular}{|l|} 
Designer/IES \\
Chair
\end{tabular} & & & & & & & $\begin{array}{l}\text { The controls templates for } \\
\text { workstation-specific are the } \\
\text { same. }\end{array}$ & \begin{tabular}{|l|} 
Controls \\
Templates
\end{tabular} & 11 & 1 & 3 & 2.6 & 4 & 5 & 4 & 4.4 & 3.3 & 2.9 \\
\hline HMcKay & HM24 & Designer & Content & & & & & & $\begin{array}{l}\text { The need for dimming } \\
\text { ballasts should be } \\
\text { mentioned in strategy } \\
\text { related to dimming. } \\
\end{array}$ & \begin{tabular}{|l|} 
Controls \\
Templates
\end{tabular} & 15 & 2 & 4 & 3.6 & 4 & 3 & 3 & 3.3 & 3.5 & 3.6 \\
\hline
\end{tabular}




\begin{tabular}{|c|c|c|c|c|c|c|c|c|c|c|c|c|c|c|c|c|c|c|c|c|}
\hline $\begin{array}{l}\text { Commenter } \\
\text { ID \# }\end{array}$ & \begin{tabular}{|l|} 
Comment \\
Reference \\
$\#$
\end{tabular} & $\begin{array}{l}\text { lommenter } \\
\text { Category }\end{array}$ & \begin{tabular}{|l} 
Comment \\
Type
\end{tabular} & Space Type & \begin{tabular}{|l|} 
Ceiling \\
Height/ \\
Total Area
\end{tabular} & \begin{tabular}{|l}
$\#$ \\
workstations/ \\
partition \\
height (open \\
office only) \\
\end{tabular} & \begin{tabular}{|l|} 
Daylight \\
Input (if \\
applicable)
\end{tabular} & $\begin{array}{l}\text { Reference webpage } \\
\text { or other location } \\
\text { (see } \\
\text { "Reference.doc") }\end{array}$ & $\begin{array}{l}\text { Comment Description: } \\
\text { Issues, Suggestions \& } \\
\text { Requests }\end{array}$ & Action/Solutior & \begin{tabular}{|l|} 
Status \\
or \\
Ticket \#
\end{tabular} & $\begin{array}{c}\text { Frequenc } \\
\mathrm{y}(20 \%)\end{array}$ & $\begin{array}{c}\text { Importance } \\
\text { of comment } \\
(80 \%)\end{array}$ & $\begin{array}{c}\text { Sum } \\
(100 \%)\end{array}$ & $\begin{array}{c}\text { Ease of } \\
\text { Implementatio } \\
\mathrm{n}(30 \%)\end{array}$ & $\begin{array}{c}\text { Duration } \\
(40 \%)\end{array}$ & $\mid \begin{array}{c}\text { Risk/ } \\
\text { Complicatio } \\
\mathrm{n}(30 \%)\end{array}$ & \begin{tabular}{|c|} 
Sum \\
$(100 \%)$
\end{tabular} & $\begin{array}{c}\text { Short Term } \\
(40 \% \\
\text { Importance) } \\
(60 \% \text { Feasibility) } \\
\end{array}$ & $\begin{array}{c}\text { Long Term } \\
(85 \% \\
\text { Importance) } \\
\text { (15\% Feasibility) } \\
\end{array}$ \\
\hline$\overline{\text { D Maniccia }}$ & DM16 & Controls & & & & & & $\begin{array}{l}\text { Large 400SF } \\
\text { conference room }\end{array}$ & $\begin{array}{l}\text { The tool allows for a } \\
\text { vacancy sensor in a very } \\
\text { large room. The sensor in } \\
\text { the wall switch would be } \\
\text { insufficient. Does this } \\
\text { make sense? If you keep } \\
\text { it, make sure to address } \\
\text { the issue in the } \\
\text { implementation guidance. }\end{array}$ & $\begin{array}{l}\text { Controls } \\
\text { Templates }\end{array}$ & 11 & 2 & 4 & 3.6 & 4 & 5 & 5 & 4.7 & 4.0 & 3.8 \\
\hline D Maniccia & DM17 & Controls & & & & & & $\begin{array}{l}\text { Meeting Room } \\
\text { Controls Template } \\
\text { 31: Vacancy } \\
\text { Sensing, Multilevel } \\
\text { (Zonal) Switching, } \\
\text { Manual Dimming }\end{array}$ & $\begin{array}{l}\text { Why have both bi-level } \\
\text { switching and manual } \\
\text { dimming in the same } \\
\text { conference room?? Do the } \\
\text { controls apply to different } \\
\text { layers of light? E.g. wall } \\
\text { washing, or general. If so, } \\
\text { that's not clear. Looking at } \\
\text { the plan view, I can see the } \\
\text { distinction, but the section } \\
\text { view doesn't show the } \\
\text { wallwashers as controlled } \\
\text { by different strategies. }\end{array}$ & \begin{tabular}{|l|} 
Controls \\
Templates \\
\end{tabular} & 11 & 2 & 4 & 3.6 & 4 & 5 & 5 & 4.7 & 4.0 & 3.8 \\
\hline J Yorgey & JY17 & Manufacturer & Functionality & & & & & $\begin{array}{l}\text { Space type } \\
\text { summarv conf room }\end{array}$ & $\begin{array}{l}\text { odd description of } \\
\text { multilevel switching and }\end{array}$ & \begin{tabular}{|l|} 
Controls \\
Templates
\end{tabular} & 11 & 2 & 4 & 3.6 & 4 & 5 & 5 & 4.7 & 4.0 & 3.8 \\
\hline M Myer & MM640 & Internal & Content & \begin{tabular}{|l|} 
Controls \\
Strategies: \\
Scheduling, \\
Daylight \\
Harvesting \\
\end{tabular} & & & & \begin{tabular}{|l} 
Approach \\
\end{tabular} & $\begin{array}{l}\text { In the last bullet are we } \\
\text { giving them local control } \\
\text { over the lighting in the } \\
\text { daylight zone? }\end{array}$ & \begin{tabular}{|l|l|l|l|l|l|l|l|} 
Controls \\
Templates
\end{tabular} & 11 & 3 & 4 & 3.6 & 4 & 5 & 5 & 4.7 & 4.0 & 3.8 \\
\hline W Dau & WD8 & \begin{tabular}{|l|}
$\begin{array}{l}\text { Designer/IES } \\
\text { Chair }\end{array}$ \\
\end{tabular} & & & & & & & $\begin{array}{l}\text { Does the scheduling } \\
\text { control strategy allow } \\
\text { dimming by schedule or is } \\
\text { it only on/off? Could that } \\
\text { be added? }\end{array}$ & \begin{tabular}{|l|} 
Controls \\
Templates
\end{tabular} & 11 & 2 & 4 & 3.6 & 4 & 5 & 5 & 4.7 & 4.0 & 3.8 \\
\hline W Dau & WD11 & \begin{tabular}{|l|} 
Designer/IES \\
Chair
\end{tabular} & & & & & & & $\begin{array}{l}\text { Private offices only give me } \\
\text { the choice of motion and } \\
\text { multi-level switching, but no } \\
\text { dimming or USL control }\end{array}$ & \begin{tabular}{|l} 
Controls \\
Templates \\
\end{tabular} & 11 & 2 & 4 & 3.6 & 4 & 5 & 5 & 4.7 & 4.0 & 3.8 \\
\hline \begin{tabular}{|l|} 
W Dau \\
\end{tabular} & WD13 & \begin{tabular}{|l|} 
Designer/IES \\
Chair
\end{tabular} & & & & & & & $\begin{array}{l}\text { why would I want multilevel } \\
\text { switching and dimming on } \\
\text { the same template option? }\end{array}$ & \begin{tabular}{|l|} 
Controls \\
Templates
\end{tabular} & 11 & 2 & 4 & 3.6 & 4 & 5 & 5 & 4.7 & 4.0 & 3.8 \\
\hline
\end{tabular}




\begin{tabular}{|c|c|c|c|c|c|c|c|c|c|c|c|c|c|c|c|c|c|c|c|c|}
\hline $\begin{array}{l}\text { Commenter } \\
\text { ID \# }\end{array}$ & \begin{tabular}{|l|} 
Comment \\
Reference \\
$\#$
\end{tabular} & $\begin{array}{l}\text { Commenter } \\
\text { Category }\end{array}$ & \begin{tabular}{|l} 
Comment \\
Type
\end{tabular} & Space Type & \begin{tabular}{|l|} 
Ceiling \\
Height/ \\
Total Area
\end{tabular} & \begin{tabular}{|l}
$\#$ \\
workstations/ \\
partition \\
height (open \\
office only) \\
\end{tabular} & $\left|\begin{array}{l}\text { Daylight } \\
\text { Input (if } \\
\text { applicable) }\end{array}\right|$ & $\begin{array}{l}\text { Reference webpage } \\
\text { or other location } \\
\text { (see } \\
\text { "Reference.doc") } \\
\end{array}$ & $\begin{array}{l}\text { Comment Description: } \\
\text { Issues, Suggestions \& } \\
\text { Requests }\end{array}$ & Action/Solution & \begin{tabular}{|l|} 
Status \\
or \\
Ticket \#
\end{tabular} & $\begin{array}{c}\text { Frequenc } \\
\mathrm{y}(20 \%)\end{array}$ & $\begin{array}{l}\text { Importance } \\
\text { of comment } \\
(80 \%)\end{array}$ & $\begin{array}{c}\text { Sum } \\
(100 \%)\end{array}$ & $\begin{array}{c}\text { Ease of } \\
\text { Implementatio } \\
\mathrm{n}(30 \%)\end{array}$ & $\begin{array}{c}\text { Duration } \\
(40 \%)\end{array}$ & $\begin{array}{c}\text { Risk/ } \\
\text { Complicatio } \\
\mathrm{n}(30 \%)\end{array}$ & $\begin{array}{c}\text { Sum } \\
(100 \%)\end{array}$ & \begin{tabular}{c|} 
Short Term \\
$(40 \%$ \\
Importance) \\
$(60 \%$ Feasibility) \\
\end{tabular} & $\begin{array}{c}\text { Long Term } \\
(85 \% \\
\text { Importance) } \\
\text { (15\% Feasibility) }\end{array}$ \\
\hline M Myer & MM660 & $\mid$ Internal & Content & $\begin{array}{l}\text { Controls } \\
\text { Strategies: } \\
\text { Vacancy } \\
\text { Sensors, } \\
\text { Daylight } \\
\text { Harvesting }\end{array}$ & & & & Strategy & $\begin{array}{l}\text { 7th bullet - the text differs } \\
\text { from the section diagram }\end{array}$ & \begin{tabular}{|l|} 
Controls \\
Templates
\end{tabular} & 16 & 2 & 4 & 3.6 & 4 & 5 & 5 & 4.7 & 4.0 & 3.8 \\
\hline M Myer & MM652 & Internal & Content & \begin{tabular}{|l|} 
Controls \\
Strategies: \\
Vacancy \\
Sensors, \\
Daylight \\
Harvesting
\end{tabular} & & & & Strategy & $\begin{array}{l} \\
\text { Text is not the same. I } \\
\text { compared the bullets in a } \\
\text { Scheduling, Daylight } \\
\text { Harvesting template and I } \\
\text { had different daylighting- } \\
\text { specific text between the } \\
\text { two templates. }\end{array}$ & \begin{tabular}{|l|} 
Controls \\
Templates
\end{tabular} & 16 & 2 & 4 & 3.6 & 4 & 5 & 5 & 4.7 & 4.0 & 3.8 \\
\hline J Yorgey & JY9 & Manufacturer & \begin{tabular}{|l|} 
Functionality \\
\end{tabular} & & & & & Control strategies & $\begin{array}{l}\text { Diagram detail too small, } \\
\text { unreadable, This one says } \\
\text { occupant switching for } \\
\text { daylight control - should } \\
\text { recommend dimming -like } \\
\text { occ sense /daylight }\end{array}$ & \begin{tabular}{|l|} 
Controls \\
Templates
\end{tabular} & 11 & 3 & 4 & 3.8 & 4 & 5 & 5 & 4.7 & 4.2 & 3.9 \\
\hline J Yorgey & JY11 & Manufacturer & Content & & & & & Control strategies & $\begin{array}{l}\text { This one says occupant } \\
\text { switching for daylight } \\
\text { control - should } \\
\text { recommend dimming -like } \\
\text { occ sense /daylight }\end{array}$ & \begin{tabular}{|l|} 
Controls \\
Templates
\end{tabular} & 11 & 3 & 4 & 3.8 & 4 & 5 & 5 & 4.7 & 4.2 & 3.9 \\
\hline HMcKay & HM2O & Designer & Content & & & & & & $\begin{array}{l}\text { "Granular" level of control } \\
\text { not meaningful to general } \\
\text { user. Sounds like grit. }\end{array}$ & \begin{tabular}{|l|} 
Controls \\
Templates
\end{tabular} & 16 & 2 & 4 & 3.6 & 5 & 5 & 5 & 5 & 4.2 & 3.8 \\
\hline
\end{tabular}




\begin{tabular}{|c|c|c|c|c|c|c|c|c|c|c|c|c|c|c|c|c|c|c|c|c|}
\hline $\begin{array}{l}\text { Commenter } \\
\text { ID \# }\end{array}$ & \begin{tabular}{|l|} 
Comment \\
Reference \\
$\#$
\end{tabular} & $\begin{array}{l}\text { Commenter } \\
\text { Category }\end{array}$ & $\begin{array}{l}\text { Comment } \\
\text { Type }\end{array}$ & Space Type & \begin{tabular}{|l|} 
Ceiling \\
Height/ \\
Total Area
\end{tabular} & \begin{tabular}{|l|}
$\#$ \\
workstations/ \\
partition \\
height (open \\
office only) \\
\end{tabular} & $\left|\begin{array}{l}\text { Daylight } \\
\text { Input (if } \\
\text { applicable) }\end{array}\right|$ & $\begin{array}{l}\text { Reference webpage } \\
\text { or other location } \\
\text { (see } \\
\text { "Reference.doc") }\end{array}$ & $\begin{array}{l}\text { Comment Description: } \\
\text { Issues, Suggestions \& } \\
\text { Requests }\end{array}$ & Action/Solution & $\begin{array}{l}\text { Status } \\
\text { or } \\
\text { Ticket \# }\end{array}$ & $\begin{array}{c}\text { Frequenc } \\
\mathrm{y}(20 \%)\end{array}$ & $\begin{array}{c}\text { Importance } \\
\text { of comment } \\
(80 \%)\end{array}$ & $\begin{array}{c}\text { Sum } \\
(100 \%)\end{array}$ & $\begin{array}{c}\text { Ease of } \\
\text { Implementatio } \\
\mathrm{n}(30 \%)\end{array}$ & $\begin{array}{c}\text { Duration } \\
(40 \%)\end{array}$ & $\begin{array}{c}\text { Risk/ } \\
\text { Complicatio } \\
\mathrm{n}(30 \%)\end{array}$ & $\begin{array}{c}\text { Sum } \\
(100 \%)\end{array}$ & $\begin{array}{c}\text { Short Term } \\
(40 \% \\
\text { Importance) } \\
\text { (60\% Feasibility) } \\
\end{array}$ & $\begin{array}{c}\text { Long Term } \\
(85 \% \\
\text { Importance) } \\
\text { (15\% Feasibility) }\end{array}$ \\
\hline HMcKay & 4 HM38.1 & $\overline{~ D e s i g n e r}$ & & & & & & & $\begin{array}{l}\text { V1- Approach: Not really a } \\
\text { how-to. Confusing if } \\
\text { you don't already know } \\
\text { how it works. "Robust" will } \\
\text { not have meaning to } \\
\text { general user. Approach tab. } \\
\text { "transparent to the } \\
\text { occupant" could be } \\
\text { confusing - transparent it } \\
\text { could mean obvious, when } \\
\text { the opposite is intended. It } \\
\text { doesn't have } \\
\text { to be undetectable, just } \\
\text { smooth and continous so } \\
\text { it is not distracting to the } \\
\text { occupants. Strategy tab - } \\
\text { many paragraphs are } \\
\text { incomplete. Control tab } \\
\text { incomplete. }\end{array}$ & \begin{tabular}{|l} 
Controls \\
Templates
\end{tabular} & 16 & 2 & 4 & 3.6 & 5 & 5 & 5 & 5 & 4.2 & 3.8 \\
\hline M Myer & MM653 & Internal & Content & \begin{tabular}{|l|} 
Controls \\
Strategies: \\
Vacancy \\
Sensors, \\
Daylight \\
Harvesting
\end{tabular} & & & & Strategy & $\begin{array}{l}\text { "highly-sensitive" should be } \\
\text { hyphenated }\end{array}$ & \begin{tabular}{|l|} 
Controls \\
Templates \\
\end{tabular} & 16 & 2 & 4 & 3.6 & 5 & 5 & 5 & 5 & 4.2 & 3.8 \\
\hline M Myer & MM639 & Internal & Content & \begin{tabular}{|l|} 
Controls \\
Strategies: \\
Scheduling, \\
Daylight \\
Harvesting
\end{tabular} & & & & Approach & $\begin{array}{l}\text { 1st bullet - emphasize } \\
\text { scheduling here. The } \\
\text { lighting should be } \\
\text { SCHEDULED to turn off } \\
\text { the lighting... }\end{array}$ & \begin{tabular}{|l|} 
Controls \\
Templates
\end{tabular} & 16 & 2 & 4 & 3.6 & 5 & 5 & 5 & 5 & 4.2 & 3.8 \\
\hline M Myer & MM655 & Internal & Content & \begin{tabular}{|l|} 
Controls \\
Strategies: \\
Vacancy \\
Sensors, \\
Daylight \\
Harvesting
\end{tabular} & & & & Strategy & $\begin{array}{l}\text { 4th bullet - I think that you } \\
\text { need more text saying that } \\
\text { those distances are in plan } \\
\text { from the edge of the } \\
\text { daylight aperture }\end{array}$ & \begin{tabular}{|l|} 
Controls \\
Templates
\end{tabular} & 16 & 2 & 4 & 3.6 & 5 & 5 & 5 & 5 & 4.2 & 3.8 \\
\hline M Myer & MM658 & Internal & Content & \begin{tabular}{|l|} 
Controls \\
Strategies: \\
Vacancy \\
Sensors, \\
Daylight \\
Harvesting
\end{tabular} & & & & Strategy & $\begin{array}{l}\text { 5th bullet - what does this } \\
\text { mean "The zones should } \\
\text { be small enough to be } \\
\text { localized" }\end{array}$ & \begin{tabular}{|l|} 
Controls \\
Templates
\end{tabular} & 16 & 2 & 4 & 3.6 & 5 & 5 & 5 & 5 & 4.2 & 3.8 \\
\hline M Myer & MM659 & $\mid$ Internal & Content & \begin{tabular}{|l|} 
Controls \\
Strategies: \\
Vacancy \\
Sensors, \\
Daylight \\
Harvesting \\
\end{tabular} & & & & Strategy & $\begin{array}{l}\text { 6th bullet - I am confused } \\
\text { by this bullet }\end{array}$ & \begin{tabular}{|l|} 
Controls \\
Templates
\end{tabular} & 16 & 2 & 4 & 3.6 & 5 & 5 & 5 & 5 & 4.2 & 3.8 \\
\hline M Myer & \begin{tabular}{|l|l|l|} 
MM648 & \\
\end{tabular} & Internal & Content & \begin{tabular}{|l|} 
Controls \\
Strategies: \\
Vacancy \\
Sensors, \\
Daylight \\
Harvesting
\end{tabular} & & & & Approach & $\begin{array}{l}\text { In the first bullet, } \\
\text { emphasize the sensors } \\
\text { turning off light when the } \\
\text { space is vacant }\end{array}$ & \begin{tabular}{|l|} 
Controls \\
Templates
\end{tabular} & 16 & 2 & 4 & 3.6 & 5 & 5 & 5 & 5 & 4.2 & 3.8 \\
\hline
\end{tabular}


Appendix E: Master Comments List

\begin{tabular}{|c|c|c|c|c|c|c|c|c|c|c|c|c|c|c|c|c|c|c|c|c|}
\hline $\begin{array}{l}\text { Commenter } \\
\text { ID \# }\end{array}$ & $\begin{array}{l}\text { Comment } \\
\text { Reference } \\
\#\end{array}$ & $\begin{array}{l}\text { Commenter } \\
\text { Category }\end{array}$ & $\begin{array}{l}\text { Comment } \\
\text { Type }\end{array}$ & Space Type & \begin{tabular}{|l|} 
Ceiling \\
Height/ \\
Total Area
\end{tabular} & \begin{tabular}{|l|}
$\#$ \\
workstations/ \\
partition \\
height (open \\
office only) \\
\end{tabular} & \begin{tabular}{|l} 
Daylight \\
Input (if \\
applicable)
\end{tabular} & $\begin{array}{l}\text { Reference webpage } \\
\text { or other location } \\
\text { (see } \\
\text { "Reference.doc") } \\
\end{array}$ & $\begin{array}{l}\text { Comment Description: } \\
\text { Issues, Suggestions \& } \\
\text { Requests }\end{array}$ & Action/Solutio & \begin{tabular}{|l|} 
Status \\
or \\
Ticket \#
\end{tabular} & $\begin{array}{c}\text { Frequenc } \\
\mathrm{y}(20 \%)\end{array}$ & $\begin{array}{c}\text { Importance } \\
\text { of comment } \\
(80 \%)\end{array}$ & $\begin{array}{c}\text { Sum } \\
(100 \%)\end{array}$ & $\begin{array}{c}\text { Ease of } \\
\text { Implementatio } \\
\mathrm{n}(30 \%)\end{array}$ & $\begin{array}{c}\text { Duration } \\
(40 \%)\end{array}$ & $\begin{array}{c}\text { Risk/ } \\
\text { Complicatio } \\
\mathrm{n}(30 \%)\end{array}$ & \begin{tabular}{|} 
Sum \\
$(100 \%)$
\end{tabular} & \begin{tabular}{|c|} 
Short Term \\
$(40 \%$ \\
Importance) \\
$(60 \%$ Feasibility)
\end{tabular} & \begin{tabular}{|c|} 
Long Term \\
(85\% \\
Importance) \\
(15\% Feasibility)
\end{tabular} \\
\hline M Myer & MM657 & |Internal & Content & $\begin{array}{l}\text { Controls } \\
\text { Strategies: } \\
\text { Vacancy } \\
\text { Sensors, } \\
\text { Daylight } \\
\text { Harvesting }\end{array}$ & & & & Strategy & \begin{tabular}{|l|} 
The 5 th bullet is worded \\
weirdly "Daylight zone can \\
be defined as about 1.5 \\
times the head height of \\
the window in from the \\
window." - height of the \\
window in from the window. \\
I think that we need to \\
reinforce this is horizontal \\
distance from the window \\
of a vertical measurement
\end{tabular} & $\begin{array}{l}\text { Controls } \\
\text { Templates }\end{array}$ & 16 & 2 & 4 & 3.6 & 5 & 5 & 5 & 5 & 4.2 & 3.8 \\
\hline M Myer & \begin{tabular}{|l|} 
MM629 \\
\end{tabular} & Internal & \begin{tabular}{|l|} 
Appearance \\
Aesthetics
\end{tabular} & $\begin{array}{l}\text { Control } \\
\text { Strategy: } \\
\text { Vacancy } \\
\text { Sensor }\end{array}$ & & & & Approach & \begin{tabular}{|l|} 
This does not sound right \\
"Vacancy sensors should \\
turn the lights off after the \\
space is unoccupied to \\
save energy, based on \\
control zones small enough \\
to
\end{tabular} & \begin{tabular}{|l|} 
Controls \\
Templates
\end{tabular} & 16 & 2 & 4 & 3.6 & 5 & 5 & 5 & 5 & 4.2 & 3.8 \\
\hline M Myer & \begin{tabular}{|l|} 
MM649 \\
\end{tabular} & Internal & \begin{tabular}{|l|} 
Content \\
\end{tabular} & \begin{tabular}{|l|} 
Controls \\
Strategies: \\
Vacancy \\
Sensors, \\
Daylight \\
Harvesting
\end{tabular} & & & & Approach & \begin{tabular}{|l|} 
Well now that I write that \\
and read the 3 bullet, I am \\
confused. Aren't the first \\
and third bullets \\
redundant?
\end{tabular} & \begin{tabular}{|l|} 
Controls \\
Templates
\end{tabular} & 16 & 2 & 4 & 3.6 & 5 & 5 & 5 & 5 & 4.2 & 3.8 \\
\hline \begin{tabular}{|l|} 
A Cortese \\
\end{tabular} & AC8 & Architect & General & & & & & & \begin{tabular}{|l|} 
Under open plan, it states \\
"control the lighting by \\
zones that are consistent \\
with daylight distribution". \\
wonder if practitioners in \\
the real world will know \\
what that means. Instead, \\
perhaps the CLS tool could \\
provide some guidance on \\
what zoning strategies \\
might work for a given \\
space configuration. Even \\
having a simple plan \\
diagram that shows \\
perimeter zones separate \\
from other zones might be \\
helpful. \\
\end{tabular} & \begin{tabular}{|l|} 
Controls \\
Templates \\
\end{tabular} & 16 & 1 & 5 & 4.2 & 5 & 5 & 5 & 5 & 4.5 & 4.3 \\
\hline HMcKay & HM12 & Designer & Content & & & & & & $\begin{array}{l} \\
\text { Total glazed area "above } \\
303 \text { AFF" - daylight not } \\
\text { usable below 30" }\end{array}$ & Daylighting & LT & 1 & 3 & 2.6 & 1 & 2 & 3 & 2 & 2.4 & 2.5 \\
\hline |J Yorgey & JY6 & Manufacturer & & & & & & \begin{tabular}{|l|}
$\begin{array}{l}\text { Space type } \\
\text { summary open plan }\end{array}$ \\
\end{tabular} & $\begin{array}{l}\text { Daylight choisce only has } \\
\text { window option no skylight } \\
\text { option }\end{array}$ & Daylighting & LT & 1 & 3 & 2.6 & 1 & 2 & 3 & 2 & 2.4 & 2.5 \\
\hline \begin{tabular}{|l} 
N Ferzacca \\
\end{tabular} & NF5 & Engineer & & & & & & & \begin{tabular}{|l|} 
Glazing manufacturers also \\
have curtain wall systems. \\
Viacon, make glazing, but \\
not assembly. Once you \\
put mullions in, VLT would \\
be less. Probably not an \\
issue for these calcs.
\end{tabular} & Daylighting & LT & 1 & 3 & 2.6 & 1 & 2 & 3 & 2 & 2.4 & 2.5 \\
\hline
\end{tabular}


Appendix E: Master Comments List

\begin{tabular}{|c|c|c|c|c|c|c|c|c|c|c|c|c|c|c|c|c|c|c|c|c|}
\hline $\begin{array}{l}\text { Commenter } \\
\text { ID \# }\end{array}$ & $\begin{array}{l}\text { Comment } \\
\text { Reference } \\
\#\end{array}$ & $\begin{array}{l}\text { Commenter } \\
\text { Category }\end{array}$ & \begin{tabular}{|l} 
Comment \\
Type
\end{tabular} & Space Type & \begin{tabular}{|l|} 
Ceiling \\
Height/ \\
Total Area
\end{tabular} & \begin{tabular}{|l}
$\#$ \\
workstations/ \\
partition \\
height (open \\
office only)
\end{tabular} & \begin{tabular}{|l} 
Daylight \\
Input (if \\
applicable)
\end{tabular} & \begin{tabular}{|l|} 
Reference webpage \\
or other location \\
(see \\
"Reference.doc") \\
\end{tabular} & $\begin{array}{l}\text { Comment Description: } \\
\text { Issues, Suggestions \& } \\
\text { Requests }\end{array}$ & Action/Solution & \begin{tabular}{|l|} 
Status \\
or \\
Ticket \#
\end{tabular} & $\begin{array}{c}\text { Frequenc } \\
y(20 \%)\end{array}$ & $\begin{array}{c}\text { Importance } \\
\text { of comment } \\
(80 \%)\end{array}$ & $\begin{array}{c}\text { Sum } \\
(100 \%)\end{array}$ & $\begin{array}{c}\text { Ease of } \\
\text { Implementatio } \\
\mathrm{n}(30 \%)\end{array}$ & $\begin{array}{c}\text { Duration } \\
(40 \%)\end{array}$ & $\begin{array}{c}\text { Risk/ } \\
\text { Complicatio } \\
\mathrm{n}(30 \%)\end{array}$ & \begin{tabular}{|} 
Sum \\
$(100 \%)$
\end{tabular} & \begin{tabular}{|c|} 
Short Term \\
$(40 \%$ \\
Importance) \\
$(60 \%$ Feasibility)
\end{tabular} & $\begin{array}{c}\text { Long Term } \\
\text { (85\% } \\
\text { Importance) } \\
\text { (15\% Feasibility) }\end{array}$ \\
\hline N Ferzacca & NF12 & Engineer & & & & & & & $\begin{array}{l}\text { Maybe this is by design, } \\
\text { but you aren't getting into } \\
\text { architecural solutions. }\end{array}$ & Daylighting & LT & 2 & 3 & 2.8 & 1 & 2 & 3 & 2 & 2.5 & 2.7 \\
\hline M Dare & MD5 & Utility & & & & & & & $\begin{array}{l}\text { I have } 3 \text { glazing walls, due } \\
\text { north, south and west. How } \\
\text { do I average that? }\end{array}$ & Daylighting & LT & 1 & 4 & 3.4 & 1 & 2 & 3 & 2 & 2.8 & 3.2 \\
\hline HMcKay & HM15 & Designer & Content & & & & & & $\begin{array}{l}\text { If user inputs some } \\
\text { ridiculous data, like a VLT } \\
\text { of } 0.1 \text { and a WWR of } 0.1 \text {, } \\
\text { there should be a pop-up } \\
\text { that says "Glazing not } \\
\text { sufficient to } \\
\text { achieve savings from } \\
\text { daylight harvesting." I } \\
\text { realize this will be handled } \\
\text { in the future. }\end{array}$ & Daylighting & LT & 1 & 3 & 2.6 & 4 & 3 & 3 & 3.3 & 2.9 & 2.7 \\
\hline \begin{tabular}{|l|} 
A Cortese \\
\end{tabular} & AC15 & Architect & General & & & & & & $\begin{array}{l}\text { Control vignettes-would } \\
\text { providing guidance on the } \\
\text { suggested location of } \\
\text { daylight controls/sensors } \\
\text { be helpful? I understand } \\
\text { this is an ongoing } \\
\text { challenge. }\end{array}$ & \begin{tabular}{|l|} 
Daylighting \\
\end{tabular} & 28 & 3 & 4 & 3.8 & 5 & 4 & 4 & 4.3 & 4.0 & 3.9 \\
\hline \begin{tabular}{|l} 
A Cortese \\
\end{tabular} & AC20 & Architect & Functionality & & & & & & $\begin{array}{l}\text { I was frustrated when I } \\
\text { erroneously entered data in } \\
\text { a "finished" space. I was } \\
\text { unable to go back and edit } \\
\text { the data. Instead I had to } \\
\text { delete the space and re- } \\
\text { enter. }\end{array}$ & Functionality & 63 & 0 & 0 & 0 & 0 & 0 & 0 & 0 & 0.0 & 0.0 \\
\hline J Yorgey & JY35 & Manufacturer & Functionality & & & & & reload of project & $\begin{array}{l}\text { no energy saings } \\
\text { information retained / } \\
\text { nothing comes up on } \\
\text { downloads / can't edit } \\
\text { spaces }\end{array}$ & Functionality & TBD & 1 & 3 & 2.6 & 2 & 2 & 3 & 2.3 & 2.5 & 2.6 \\
\hline A Cortese & $\mid \mathrm{AC22}$ & Architect & Functionality & & & & & & $\begin{array}{l}\text { After I entered the open } \\
\text { office glazing of "34.5", I } \\
\text { continually received the } \\
\text { following error message } \\
\text { and was never able to } \\
\text { change or finish the project. } \\
\text { The error read: "Error } \\
\text { marshalling java.util.sorted } \\
\text { set: Error marshalling } \\
\text { java.lang.integer: Format } \\
\text { error converting } 34.5 \text { See } \\
\text { the Log for more details } \\
\text { See the log for even more } \\
\text { details" }\end{array}$ & Functionality & TBD & 1 & 3 & 2.6 & 3 & 3 & 3 & 3 & 2.8 & 2.7 \\
\hline E Smith & ES3 & Owner & & & & & & & $\begin{array}{l}\text { When I went back to } \\
\text { change operating hours, it } \\
\text { wouldn't take the change or } \\
\text { change the energy savings } \\
\text { bar at the bottom. }\end{array}$ & Functionality & TBD & 1 & 5 & 4.2 & 2 & 2 & 3 & 2.3 & 3.4 & 3.9 \\
\hline K Abernathy & KA5 & Designer & General & Pharmacy & & & & \begin{tabular}{|l|} 
Luminaires Parallel \\
to Wall Pharmacy - \\
under Approach
\end{tabular} & $\begin{array}{l}\text { Random bullet point makes } \\
\text { it feel like one is missing } \\
\text { something. }\end{array}$ & Functionality & 21 & 2 & 4 & 3.6 & 3 & 3 & 5 & 3.6 & 3.6 & 3.6 \\
\hline
\end{tabular}




\begin{tabular}{|c|c|c|c|c|c|c|c|c|c|c|c|c|c|c|c|c|c|c|c|c|}
\hline $\begin{array}{l}\text { Commenter } \\
\text { ID \# }\end{array}$ & \begin{tabular}{|l|} 
Comment \\
Reference \\
$\#$
\end{tabular} & $\begin{array}{l}\text { Commenter } \\
\text { Category }\end{array}$ & \begin{tabular}{|l} 
Comment \\
Type
\end{tabular} & Space Type & \begin{tabular}{|l|} 
Ceiling \\
Height/ \\
Total Area
\end{tabular} & \begin{tabular}{|l}
$\#$ \\
workstations/ \\
partition \\
height (open \\
office only) \\
\end{tabular} & \begin{tabular}{|l|} 
Daylight \\
Input (if \\
applicable)
\end{tabular} & \begin{tabular}{|l|} 
Reference webpage \\
or other location \\
(see \\
"Reference.doc")
\end{tabular} & $\begin{array}{l}\text { Comment Description: } \\
\text { Issues, Suggestions \& } \\
\text { Requests }\end{array}$ & Action/Solution & \begin{tabular}{|l|} 
Status \\
or \\
Ticket \#
\end{tabular} & $\begin{array}{c}\text { Frequenc } \\
\mathrm{y}(20 \%)\end{array}$ & $\begin{array}{c}\text { Importance } \\
\text { of comment } \\
(80 \%)\end{array}$ & \begin{tabular}{|c|} 
Sum \\
$(100 \%)$
\end{tabular} & $\begin{array}{c}\text { Ease of } \\
\text { Implementatio } \\
\mathrm{n}(30 \%)\end{array}$ & $\begin{array}{c}\text { Duration } \\
(40 \%)\end{array}$ & $\begin{array}{c}\text { Risk/ } \\
\text { Complicatio } \\
\mathrm{n}(30 \%)\end{array}$ & \begin{tabular}{|c|} 
Sum \\
$(100 \%)$
\end{tabular} & $\begin{array}{c}\text { Short Term } \\
(40 \% \\
\text { Importance) } \\
(60 \% \text { Feasibility) } \\
\end{array}$ & $\begin{array}{c}\text { Long Term } \\
(85 \% \\
\text { Importance) } \\
\text { (15\% Feasibility) } \\
\end{array}$ \\
\hline M Myer & 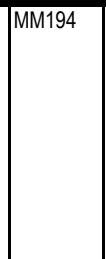 & Internal & Content & \begin{tabular}{|l|} 
Recessed slot \\
luminaires \\
Perpendicular \\
to Desk with \\
Compact \\
Fluorescent \\
Downlights and \\
Wall-washers \\
(Office)
\end{tabular} & & & & Strategy/daylighting & $\begin{array}{l}\text { If no daylighting text is } \\
\text { presented, I would remove } \\
\text { this heading }\end{array}$ & Functionality & 21 & 2 & 4 & 3.6 & 3 & 3 & 5 & 3.6 & 3.6 & 3.6 \\
\hline N Miller & NM16 & Internal & & & & & & & $\begin{array}{l}\text { there is a random bullet } \\
\text { point }\end{array}$ & \begin{tabular}{|l|} 
Functionality \\
\end{tabular} & 21 & 2 & 4 & 3.6 & 3 & 3 & 5 & 3.6 & 3.6 & 3.6 \\
\hline N Miller & NM9.1 & Internal & & & & & & & Extra bullet in Concept text. & Functionality & 21 & 2 & 4 & 3.6 & 3 & 3 & 5 & 3.6 & 3.6 & 3.6 \\
\hline C Magee & CM4 & Owner & & & & & & & $\begin{array}{l}\text { Graphics are definitely not } \\
\text { showing up correctly. }\end{array}$ & Functionality & TBD & 1 & 5 & 4.2 & 3 & 3 & 3 & 3 & 3.7 & 4.0 \\
\hline C DiLouie & CD11 & Core Team & Content & & & & & & $\begin{array}{l}\text { I went through private } \\
\text { office, chose my lighting } \\
\text { template and controls, and } \\
\text { got } 172 \% \text { energy savings }\end{array}$ & Functionality & 20 & 2 & 5 & 4.4 & 3 & 3 & 5 & 3.6 & 4.1 & 4.3 \\
\hline \begin{tabular}{|l|l} 
L Davis \\
\end{tabular} & LD14 & Designer & & & & & & & $\begin{array}{l}\text { When I got back to key } \\
\text { plan, the energy savings } \\
\text { showed } 845 \% \text { savings. } \\
\text { The proposed savings } \\
\text { showed NEGATIVE kWh } \\
\text { savings }\end{array}$ & Functionality & 20 & 2 & 5 & 4.4 & 3 & 3 & 5 & 3.6 & 4.1 & 4.3 \\
\hline A Cortese & AC19 & Architect & \begin{tabular}{|l|} 
Functionality \\
\end{tabular} & & & & & & $\begin{array}{l}\text { The system would only } \\
\text { allow me to save one } \\
\text { project. My second working } \\
\text { example was never able to } \\
\text { be saved for future } \\
\text { reference. At first I thought } \\
\text { this was a "time out" } \\
\text { problem, but I was never } \\
\text { able to save a second } \\
\text { project. }\end{array}$ & Functionality & 64 & 2 & 5 & 4.4 & 3 & 4 & 5 & 4 & 4.2 & 4.3 \\
\hline A Cortese & AC23 & Architect & \begin{tabular}{|l|} 
Functionality \\
\end{tabular} & & & & & & \begin{tabular}{|l|} 
Open Plan offices - \\
Despite the instructions to \\
the contrary, 0 is not an \\
option for "no partitions" \\
under partition height. I \\
ended up using 1 instead.
\end{tabular} & \begin{tabular}{|l|} 
Functionality \\
\end{tabular} & 65 & 2 & 5 & 4.4 & 3 & 4 & 5 & 4 & 4.2 & 4.3 \\
\hline \begin{tabular}{|l|l|} 
C DiLouie \\
\end{tabular} & CD1 & Core Team & \begin{tabular}{|l|} 
Functionality \\
\end{tabular} & & & & & & $\begin{array}{l}\text { on the opening screen, } \\
\text { some weird text appears on } \\
\text { the far left of my } \\
\text { screen when I have the } \\
\text { webpage take up my entire } \\
\text { screen. The } \\
\text { text appears using Google } \\
\text { Chrome, Firefox and } \\
\text { Internet Explorer. }\end{array}$ & Functionality & 66 & 2 & 5 & 4.4 & 3 & 4 & 5 & 4 & 4.2 & 4.3 \\
\hline C Magee & CM1 & Owner & & & & & & & $\begin{array}{l}\text { When I clicked the new } \\
\text { project button the first time } \\
\text { I got an error, the second } \\
\text { time it worked. }\end{array}$ & \begin{tabular}{|l|} 
Functionality \\
\end{tabular} & 67 & 2 & 5 & 4.4 & 3 & 4 & 5 & 4 & 4.2 & 4.3 \\
\hline
\end{tabular}


Appendix E: Master Comments List

\begin{tabular}{|c|c|c|c|c|c|c|c|c|c|c|c|c|c|c|c|c|c|c|c|c|}
\hline $\begin{array}{l}\text { Commenter } \\
\text { ID \# }\end{array}$ & $\begin{array}{l}\text { Comment } \\
\text { Reference } \\
\#\end{array}$ & $\begin{array}{l}\text { Commenter } \\
\text { Category }\end{array}$ & $\begin{array}{l}\text { Comment } \\
\text { Type }\end{array}$ & Space Type & \begin{tabular}{|l|} 
Ceiling \\
Height/ \\
Total Area
\end{tabular} & \begin{tabular}{|l|}
$\#$ \\
workstations/ \\
partition \\
height (open \\
office only) \\
\end{tabular} & \begin{tabular}{|l} 
Daylight \\
Input (if \\
applicable)
\end{tabular} & \begin{tabular}{|l|} 
Reference webpage \\
or other location \\
(see \\
"Reference.doc")
\end{tabular} & $\begin{array}{l}\text { Comment Description: } \\
\text { Issues, Suggestions \& } \\
\text { Requests }\end{array}$ & Action/Solution & \begin{tabular}{|l|} 
Status \\
or \\
Ticket \#
\end{tabular} & $\begin{array}{c}\text { Frequenc } \\
\mathrm{y}(20 \%)\end{array}$ & $\begin{array}{c}\text { Importance } \\
\text { of comment } \\
(80 \%)\end{array}$ & \begin{tabular}{|c|} 
Sum \\
$(100 \%)$
\end{tabular} & $\begin{array}{c}\text { Ease of } \\
\text { Implementatio } \\
\mathrm{n}(30 \%)\end{array}$ & $\begin{array}{c}\text { Duration } \\
(40 \%)\end{array}$ & $\begin{array}{c}\text { Risk/ } \\
\text { Complicatio } \\
\mathrm{n}(30 \%)\end{array}$ & \begin{tabular}{|} 
Sum \\
$(100 \%)$
\end{tabular} & $\begin{array}{c}\text { Short Term } \\
(40 \% \\
\text { Importance) } \\
(60 \% \text { Feasibility) }\end{array}$ & \begin{tabular}{|c|} 
Long Term \\
(85\% \\
Importance) \\
(15\% Feasibility)
\end{tabular} \\
\hline KAbernathy & KA11 & Designer & & & & & & & \begin{tabular}{|l|} 
Web-site crashed before I \\
could get to my results. My \\
browser may have been \\
having issues. I constantly \\
had to override the security \\
certificate and perhaps that \\
was because it is a beta \\
version.
\end{tabular} & Functionality & 67 & 2 & 5 & 4.4 & 3 & 4 & 5 & 4 & 4.2 & 4.3 \\
\hline M Dare & MD12 & Utility & & & & & & & \begin{tabular}{|l|} 
got a server error after \\
looking at the summary \\
screen after picking my \\
vignettes, and hitting next.
\end{tabular} & \begin{tabular}{|l} 
Functionality \\
\end{tabular} & 67 & 2 & 5 & 4.4 & 3 & 4 & 5 & 4 & 4.2 & 4.3 \\
\hline R Parish & RP1 & Owner & & & & & & & $\begin{array}{l}\text { Not found error, over and } \\
\text { over again, had to stop } \\
\text { using the tool and have } \\
\text { Carol do it. }\end{array}$ & Functionality & 67 & 2 & 5 & 4.4 & 3 & 4 & 5 & 4 & 4.2 & 4.3 \\
\hline W Dau & WD14 & \begin{tabular}{|l} 
Designer/IES \\
Chair
\end{tabular} & & & & & & & \begin{tabular}{|l|} 
server error when \\
generating the final report \\
"the page at \\
https://beta.lighting- \\
solutions.org says: A server \\
error has ocurred"
\end{tabular} & Functionality & 67 & 2 & 5 & 4.4 & 3 & 4 & 5 & 4 & 4.2 & 4.3 \\
\hline $\begin{array}{l}\text { C Magee } \\
\text {. }\end{array}$ & CM2 & Owner & & & & & & & $\begin{array}{l}\text { Input box for reception } \\
\text { square footage was } \\
\text { missing, then when I hit } \\
\text { next it showed up and I } \\
\text { could type in the SF-age. }\end{array}$ & Functionality & 68 & 2 & 5 & 4.4 & 3 & 4 & 5 & 4 & 4.2 & 4.3 \\
\hline HMcKay & HM36 & & Functionality & & & & & & \begin{tabular}{|l|} 
Template 1 - lines above \\
the title "Most flexible ...." \\
Increases and decreases \\
when mouse is moved over \\
page.
\end{tabular} & Functionality & 68 & 2 & 5 & 4.4 & 3 & 4 & 5 & 4 & 4.2 & 4.3 \\
\hline J Yorgey & JY5 & Manufacturer & Functionality & & & & & \begin{tabular}{|l|} 
Space type \\
summary open plan
\end{tabular} & $\begin{array}{l}\text { After adding ceiling ht. The } \\
\text { page format changed } \\
\text { \#workstaions box moves }\end{array}$ & Functionality & 68 & 2 & 5 & 4.4 & 3 & 4 & 5 & 4 & 4.2 & 4.3 \\
\hline J Yorgey & JYY27 & Manufacturer & & & & & & Energy estimate & \begin{tabular}{|l|} 
Did not work - asked to fill \\
in red fields - none wre \\
shown
\end{tabular} & \begin{tabular}{|l} 
Functionality \\
\end{tabular} & 68 & 2 & 5 & 4.4 & 3 & 4 & 5 & 4 & 4.2 & 4.3 \\
\hline $\begin{array}{l}\text { C Magee } \\
\end{array}$ & CM6 & Owner & & & & & & & $\begin{array}{l}\text { Summary screen is not } \\
\text { reflecting the values that I } \\
\text { typed in originally. E.g., I } \\
\text { put in a } 16^{\prime}-0 " \text { ceiling height } \\
\text { and it shows } 8^{\prime}-0 " \text {. }\end{array}$ & Functionality & 69 & 2 & 5 & 4.4 & 3 & 4 & 5 & 4 & 4.2 & 4.3 \\
\hline J Yorgey & JY30 & Manufacturer & Functionality & & & & & \begin{tabular}{|l|} 
Office design \\
vinettes -open plan \\
spaces
\end{tabular} & $\begin{array}{l}\text { No way to go back to edit } \\
\text { the space and the } \\
\text { information lists "0" as SqFt } \\
\text { should be } 2000\end{array}$ & Functionality & 69 & 2 & 5 & 4.4 & 3 & 4 & 5 & 4 & 4.2 & 4.3 \\
\hline J Yorgey & JY17.1 & Manufacturer & Functionality & & & & & \begin{tabular}{|l|} 
Space type \\
summary conf room
\end{tabular} & did not list \# rooms & \begin{tabular}{|l|l|} 
Functionality \\
\end{tabular} & 69 & 2 & 5 & 4.4 & 3 & 4 & 5 & 4 & 4.2 & 4.3 \\
\hline J Yorgey & JY33 & Manufacturer & Functionality & & & & & \begin{tabular}{|l|} 
Office design \\
vinettes -Edit onen
\end{tabular} & $\begin{array}{l}\text { on Details page which } \\
\text { Open Plan space is this }\end{array}$ & Functionality & 70 & 2 & 5 & 4.4 & 3 & 4 & 5 & 4 & 4.2 & 4.3 \\
\hline J Yorgey & JY18 & Manufacturer & Functionality & & & & & \begin{tabular}{|l|} 
space type \\
summary perimeter \\
office
\end{tabular} & $\begin{array}{l}\text { Pull down of "what open } \\
\text { plan associated with" does } \\
\text { not work length of walls } \\
\text { does not show in summary }\end{array}$ & Functionality & 70 & 2 & 5 & 4.4 & 3 & 4 & 5 & 4 & 4.2 & 4.3 \\
\hline |J Yorgey & JY32 & Manufacturer & Functionality & & & & & \begin{tabular}{|l|} 
Office design \\
vinettes -Edit open \\
plan perimenter \\
spaces
\end{tabular} & \begin{tabular}{|l|} 
got stuck at select \\
appropriate design \\
vignettee for this space. \\
Retrieving vignettes \\
\end{tabular} & Functionality & 71 & 2 & 5 & 4.4 & 3 & 4 & 5 & 4 & 4.2 & 4.3 \\
\hline
\end{tabular}


Appendix E: Master Comments List

\begin{tabular}{|c|c|c|c|c|c|c|c|c|c|c|c|c|c|c|c|c|c|c|c|c|}
\hline $\begin{array}{l}\text { Commenter } \\
\text { ID \# }\end{array}$ & $\begin{array}{l}\text { Comment } \\
\text { Reference } \\
\#\end{array}$ & $\begin{array}{l}\text { Commenter } \\
\text { Category }\end{array}$ & $\begin{array}{l}\text { Comment } \\
\text { Type }\end{array}$ & Space Type & \begin{tabular}{|l|} 
Ceiling \\
Height/ \\
Total Area
\end{tabular} & \begin{tabular}{|l|}
$\#$ \\
workstations/ \\
partition \\
height (open \\
office only) \\
\end{tabular} & \begin{tabular}{|l} 
Daylight \\
Input (if \\
applicable)
\end{tabular} & \begin{tabular}{|l|} 
Reference webpage \\
or other location \\
(see \\
"Reference.doc")
\end{tabular} & $\begin{array}{l}\text { Comment Description: } \\
\text { Issues, Suggestions \& } \\
\text { Requests }\end{array}$ & Action/Solution & \begin{tabular}{|l|} 
Status \\
or \\
Ticket \#
\end{tabular} & $\begin{array}{c}\text { Frequenc } \\
y(20 \%)\end{array}$ & $\begin{array}{c}\text { Importance } \\
\text { of comment } \\
(80 \%)\end{array}$ & $\begin{array}{c}\text { Sum } \\
(100 \%)\end{array}$ & $\begin{array}{c}\text { Ease of } \\
\text { Implementatio } \\
\mathrm{n}(30 \%)\end{array}$ & $\mid \begin{array}{c}\text { Duration } \\
(40 \%)\end{array}$ & $\begin{array}{c}\text { Risk/ } \\
\text { Complicatio } \\
\mathrm{n}(30 \%)\end{array}$ & \begin{tabular}{|c|} 
Sum \\
$(100 \%)$
\end{tabular} & \begin{tabular}{|c|} 
Short Term \\
$(40 \%$ \\
Importance) \\
$(60 \%$ Feasibility) \\
\end{tabular} & $\begin{array}{c}\text { Long Term } \\
\text { (85\% } \\
\text { Importance) } \\
\text { (15\% Feasibility) } \\
\end{array}$ \\
\hline J Yorgey & JY34 & Manufacturer & Functionality & & & & & \begin{tabular}{|l|}
$\begin{array}{l}\text { Office design } \\
\text { vinettes -add } \\
\text { reception spaces }\end{array}$ \\
\end{tabular} & $\begin{array}{l}\text { retrieving vignettes ... } \\
\text { never happened }\end{array}$ & Functionality & 71 & 2 & 5 & 4.4 & 3 & 4 & 5 & 4 & 4.2 & 4.3 \\
\hline C DiLouie & CD6 & Core Team & Functionality & & & & & & $\begin{array}{l}\text { https problem still there: } \\
\text { When I go to the website, } \\
\text { my browser tells me it's not } \\
\text { safe. }\end{array}$ & Functionality & 59 & 3 & 5 & 4.6 & 4 & 4 & 5 & 4.3 & 4.5 & 4.6 \\
\hline |J Yorgey & JY1 & Manufacturer & Functionality & Office & & & & Website & $\begin{array}{l}\text { Iget a security certificate } \\
\text { error when going to the } \\
\text { website. It may be our } \\
\text { filters at Lutron but It's a } \\
\text { very unusual occurrence } \\
\text { with any other website. } \\
\end{array}$ & Functionality & 59 & 3 & 5 & 4.6 & 4 & 4 & 5 & 4.3 & 4.5 & 4.6 \\
\hline L Davis & LD1 & Designer & & & & & & & $\begin{array}{l}\text { Is there a way to add a } \\
\text { security clearance so that } \\
\text { Firefox doesn't show the } \\
\text { site as untrusted? }\end{array}$ & Functionality & 59 & 3 & 5 & 4.6 & 4 & 4 & 5 & 4.3 & 4.5 & 4.6 \\
\hline \begin{tabular}{|l} 
C DiLouie \\
\end{tabular} & CD31 & Core Team & Functionality & & & & & & \begin{tabular}{|l|} 
Text is routinely cut off mid \\
paragraph/mid sentence, \\
made bullets instead of \\
sentences or sentences \\
instead of bullets, and \\
spaced unevenly line by \\
line; the text should be \\
revised line by line and \\
cleaned up. \\
\end{tabular} & Functionality & 19 & 5 & 5 & 5 & 4 & 5 & 5 & 4.7 & 4.9 & 5.0 \\
\hline \begin{tabular}{|l} 
C DiLouie \\
\end{tabular} & CD22 & Core Team & Functionality & & & & & & \begin{tabular}{|l|} 
In a few pages I looked at, \\
various control \\
implementation instructions \\
were cut off mid sentence, \\
bullets appear to be \\
chopped off for some \\
reason, meaning \\
information is missing \\
\end{tabular} & Functionality & 19 & 5 & 5 & 5 & 4 & 5 & 5 & 4.7 & 4.9 & 5.0 \\
\hline K Abernathy & KA1 & Designer & General & Pharmacy & & & & \begin{tabular}{|l|} 
Luminaires Parallel \\
to Wall with \\
Skylights Pharmacy \\
under strategy tab
\end{tabular} & $\begin{array}{l}\text { last sentence - ends with } \\
\text { ceil, I think it needs to be } \\
\text { ceiling }\end{array}$ & Functionality & 19 & 5 & 5 & 5 & 4 & 5 & 5 & 4.7 & 4.9 & 5.0 \\
\hline K Abernathy & KA6 & Designer & General & Pharmacy & & & & \begin{tabular}{|l|} 
Luminaires Parallel \\
to Wall Pharmacy - \\
under strategy tab
\end{tabular} & $\begin{array}{l}\text { last sentence - ends with } \\
\text { tubu, I think this sentence is } \\
\text { getting cut short. }\end{array}$ & Functionality & 19 & 5 & 5 & 5 & 4 & 5 & 5 & 4.7 & 4.9 & 5.0 \\
\hline M Myer & MM654 & Internal & \begin{tabular}{|l|} 
Content \\
\end{tabular} & \begin{tabular}{|l|} 
Controls \\
Strategies: \\
Vacancy \\
Sensors, \\
Daylight \\
Harvesting \\
\end{tabular} & & & & Strategy & $\begin{array}{l}\text { MISSING/INCOMPLETE } \\
\text { TEXT: "switching, ensure } \\
\text { accurate detection and } \\
\text { avoid false triggering. As } \\
\text { partition heights increase, } \\
\text { more overlap an" } \\
\end{array}$ & Functionality & 19 & 5 & 5 & 5 & 4 & 5 & 5 & 4.7 & 4.9 & 5.0 \\
\hline N Miller & NM14 & Internal & & & & & & & $\begin{array}{l}\text { the last sentence under } \\
\text { the strategy tab is falling } \\
\text { short. }\end{array}$ & Functionality & 19 & 5 & 5 & 5 & 4 & 5 & 5 & 4.7 & 4.9 & 5.0 \\
\hline
\end{tabular}




\begin{tabular}{|c|c|c|c|c|c|c|c|c|c|c|c|c|c|c|c|c|c|c|c|c|}
\hline $\begin{array}{l}\text { Commenter } \\
\text { ID \# }\end{array}$ & $\begin{array}{l}\text { Comment } \\
\text { Reference } \\
\#\end{array}$ & $\begin{array}{l}\text { Commenter } \\
\text { Category }\end{array}$ & $\begin{array}{l}\text { Comment } \\
\text { Type }\end{array}$ & Space Type & \begin{tabular}{|l} 
Ceiling \\
Height// \\
Total Area
\end{tabular} & $\begin{array}{l}\# \\
\text { workstations/ } \\
\text { partition } \\
\text { height (open } \\
\text { office only) } \\
\end{array}$ & $\mid \begin{array}{l}\text { Daylight } \\
\text { Input (if } \\
\text { applicable) }\end{array}$ & \begin{tabular}{|l|} 
Reference webpage \\
or other location \\
(see \\
"Reference.doc")
\end{tabular} & \begin{tabular}{|l|} 
Comment Description: \\
Issues, Suggestions \& \\
Requests
\end{tabular} & Action/Solution & \begin{tabular}{|l} 
Status \\
or \\
Ticket \#
\end{tabular} & $\begin{array}{c}\text { Frequenc } \\
\text { y (20\%) }\end{array}$ & $\begin{array}{c}\text { Importance } \\
\text { of comment } \\
(80 \%)\end{array}$ & \begin{tabular}{|c|} 
Sum \\
$(100 \%)$
\end{tabular} & $\begin{array}{c}\text { Ease of } \\
\text { Implementatio } \\
\mathrm{n}(30 \%)\end{array}$ & $\begin{array}{c}\text { Duration } \\
(40 \%)\end{array}$ & $\begin{array}{c}\text { Risk/ } \\
\text { Complicatio } \\
\mathrm{n}(30 \%)\end{array}$ & \begin{tabular}{|c|} 
Sum \\
$(100 \%)$
\end{tabular} & $\begin{array}{c}\begin{array}{c}\text { Short Term } \\
(40 \% \\
\text { Importance) } \\
(60 \% \text { Feasibility) }\end{array} \\
\end{array}$ & $\begin{array}{c}\text { Long Term } \\
(85 \% \\
\text { Importance) } \\
\text { (15\% Feasibility) }\end{array}$ \\
\hline N Miller & NM25 & Internal & & & & & & & $\begin{array}{l}\text { When I clicked got to the } \\
\text { details, after signing in } \\
\text { again, there is an extra } \\
\text { bullet in the first paragraph } \\
\text { of Concept-approach, and } \\
\text { some missing text in the } \\
\text { last paragraph of Strategy- } \\
\text { Dayltg. }\end{array}$ & Functionality & 19 & 5 & 5 & 5 & 4 & 5 & 5 & 4.7 & 4.9 & 5.0 \\
\hline M Myer & MM647 & Internal & Content & \begin{tabular}{|l|} 
Controls \\
Strategies: \\
Vacancy \\
Sensors, \\
Daylight \\
Harvesting \\
\end{tabular} & & & & Section Diagram & $\begin{array}{l}\text { Your light switch is too low. } \\
\text { It should be above the door } \\
\text { handle not at the same } \\
\text { height. }\end{array}$ & Images & 13 & 1 & 3 & 2.6 & 1 & 3 & 5 & 3 & 2.8 & 2.7 \\
\hline N Miller & NM8 & Internal & \begin{tabular}{|l|} 
Appearancel \\
Aesthetics
\end{tabular} & \begin{tabular}{|l|} 
Pharmacy Des \\
Vignettes
\end{tabular} & & & & \begin{tabular}{|l} 
Vignettes for \\
Perimeter
\end{tabular} & $\begin{array}{l}\text { Thumbnails of perspectives } \\
\text { too small to distinguish } \\
\text { between the two options. }\end{array}$ & Images & 13 & 1 & 3 & 2.6 & 3 & 3 & 3 & 3 & 2.8 & 2.7 \\
\hline HMcKay & HM39 & & Content & & & & & & $\begin{array}{l}\text { Control Template } 2 \text { for } \\
\text { vignette } 1 \text { - Photosensor } \\
\text { location is questionable. } \\
\text { Should be on ceiling } \\
\text { looking toward window. }\end{array}$ & Images & 13 & 2 & 2 & 2 & 4 & 4 & 5 & 4.3 & 2.9 & 2.3 \\
\hline M Myer & MM645 & Internal & Content & \begin{tabular}{|l|} 
Controls \\
Strategies: \\
Vacancy \\
Sensors, \\
Daylight \\
Harvesting \\
\end{tabular} & & & & Section Diagram & $\begin{array}{l}\text { Your photosensor is placed } \\
\text { there? I think that would } \\
\text { cause some issues. }\end{array}$ & Images & 13 & 2 & 2 & 2 & 4 & 4 & 5 & 4.3 & 2.9 & 2.3 \\
\hline HMcKay & HM40 & Designer & Content & & & & & & \begin{tabular}{|l|} 
Differences in plan and \\
perspective are noticeable. \\
Perspective has glass \\
doors and solid wall on the \\
right, but plan has no door \\
and \\
glass walls. Plan has \\
alcove between two \\
cabinets, and continuous \\
line of light within alcove, \\
but perspective shows a \\
door and plant \\
and the light fixture floating \\
in the ceiling. Plan shows \\
center row of lights \\
centered over receptionist \\
and logo, perspective does \\
not.
\end{tabular} & Images & 13 & 1 & 3 & 2.6 & 3 & 4 & 4 & 3.7 & 3.0 & 2.8 \\
\hline
\end{tabular}




\begin{tabular}{|c|c|c|c|c|c|c|c|c|c|c|c|c|c|c|c|c|c|c|c|c|}
\hline $\begin{array}{l}\text { Commenter } \\
\text { ID \# }\end{array}$ & $\begin{array}{l}\text { Comment } \\
\text { Reference } \\
\#\end{array}$ & $\begin{array}{l}\text { Commenter } \\
\text { Category }\end{array}$ & $\begin{array}{l}\text { Comment } \\
\text { Type }\end{array}$ & Space Type & \begin{tabular}{|l|} 
Ceiling \\
Height/ \\
Total Area
\end{tabular} & \begin{tabular}{|l}
$\#$ \\
workstations/ \\
partition \\
height (open \\
office only) \\
\end{tabular} & $\left|\begin{array}{l}\text { Daylight } \\
\text { Input (if } \\
\text { applicable) }\end{array}\right|$ & \begin{tabular}{|l|} 
Reference webpage \\
or other location \\
(see \\
"Reference.doc")
\end{tabular} & $\begin{array}{l}\text { Comment Description: } \\
\text { Issues, Suggestions \& } \\
\text { Requests }\end{array}$ & Action/Solution & \begin{tabular}{|l|} 
Status \\
or \\
Ticket \#
\end{tabular} & $\begin{array}{c}\text { Frequenc } \\
y(20 \%)\end{array}$ & $\begin{array}{c}\text { Importance } \\
\text { of comment } \\
(80 \%)\end{array}$ & \begin{tabular}{|c|} 
Sum \\
$(100 \%)$
\end{tabular} & $\begin{array}{c}\text { Ease of } \\
\text { Implementatio } \\
\mathrm{n}(30 \%)\end{array}$ & $\begin{array}{c}\text { Duration } \\
(40 \%)\end{array}$ & $\begin{array}{c}\text { Risk/ } \\
\text { Complicatio } \\
\mathrm{n}(30 \%)\end{array}$ & \begin{tabular}{|} 
Sum \\
$(100 \%)$
\end{tabular} \mid & \begin{tabular}{|c|} 
Short Term \\
$(40 \%$ \\
Importance) \\
$(60 \%$ Feasibility) \\
\end{tabular} & $\begin{array}{c}\text { Long Term } \\
(85 \% \\
\text { Importance) } \\
\text { (15\% Feasibility) }\end{array}$ \\
\hline M Myer & MM20 & | Internal & \begin{tabular}{|l} 
Appearancel \\
Aesthetics
\end{tabular} & $\begin{array}{l}\text { Suspended } \\
\text { Direct/lndirect } \\
\text { Luminaires, } \\
\text { Linear Wall- } \\
\text { washers, and a } \\
\text { Whiteboard } \\
\text { Light }\end{array}$ & \begin{tabular}{|l|}
$\begin{array}{l}\text { Conferenc } \\
\text { e room }\end{array}$ \\
\end{tabular} & & & Perspective & $\begin{array}{l}\text { The "bump-outs" in the wall } \\
\text { are missing in the } \\
\text { perspective that are shown } \\
\text { in the overhead. }\end{array}$ & Images & 13 & 1 & 3 & 2.6 & 3 & 4 & 4 & 3.7 & 3.0 & 2.8 \\
\hline N Miller & NM22 & Internal & & & & & & & \begin{tabular}{|l|} 
Sketch doesn't illustrate \\
column location, and the \\
window shown in the \\
sketch is not shown in the \\
overhead plan.
\end{tabular} & Images & 13 & 1 & 3 & 2.6 & 3 & 4 & 4 & 3.7 & 3.0 & 2.8 \\
\hline A Cortese & AC13 & Architect & Content & & & & & & \begin{tabular}{|l|} 
\\
On many vignettes, \\
"articulated desktop \\
luminaires" are mentioned \\
by not shown in the \\
diagram or plan.
\end{tabular} & Images & 13 & 3 & 4 & 3.8 & 4 & 4 & 5 & 4.3 & 4.0 & 3.9 \\
\hline HMcKay & HM26 & Designer & Content & & & & & & \begin{tabular}{|l|} 
\\
Non-planar vignette details: \\
No luminaire sidebar info. \\
Perspective of luminaire \\
makes it look like a planar \\
lens.
\end{tabular} & Images & 13 & 3 & 4 & 3.8 & 4 & 4 & 5 & 4.3 & 4.0 & 3.9 \\
\hline HMcKay & HM44 & Designer & Content & & & & & & \begin{tabular}{|l|} 
\\
V2 - Dropped decorative \\
diffuser. Perspective looks \\
like ordinary downlights \\
-dropped dish is not \\
apparent. No control \\
strategy?
\end{tabular} & Images & 13 & 3 & 4 & 3.8 & 4 & 4 & 5 & 4.3 & 4.0 & 3.9 \\
\hline HMcKay & HM32 & Designer & Content & & & & & & $\begin{array}{l}\text { V3 - Perspective wrong - } \\
\text { shows six 2x4s parallel to } \\
\text { conf table. }\end{array}$ & Images & 13 & 3 & 4 & 3.8 & 4 & 4 & 5 & 4.3 & 4.0 & 3.9 \\
\hline
\end{tabular}




\begin{tabular}{|c|c|c|c|c|c|c|c|c|c|c|c|c|c|c|c|c|c|c|c|c|}
\hline $\begin{array}{l}\text { Commenter } \\
\text { ID \# }\end{array}$ & $\begin{array}{l}\text { Comment } \\
\text { Reference } \\
\#\end{array}$ & $\begin{array}{l}\text { Commenter } \\
\text { Category }\end{array}$ & $\begin{array}{l}\text { Comment } \\
\text { Type }\end{array}$ & Space Type & \begin{tabular}{|l} 
Ceiling \\
Height/ \\
Total Area
\end{tabular} & \begin{tabular}{|l}
$\#$ \\
workstations/ \\
partition \\
height (open \\
office only) \\
\end{tabular} & $\mid \begin{array}{l}\text { Daylight } \\
\text { Input (if } \\
\text { applicable) }\end{array}$ & \begin{tabular}{|l|} 
Reference webpage \\
or other location \\
(see \\
"Reference.doc")
\end{tabular} & $\begin{array}{l}\text { Comment Description: } \\
\text { Issues, Suggestions \& } \\
\text { Requests }\end{array}$ & Action/Solution & \begin{tabular}{|l|}
$\begin{array}{l}\mid l \\
\text { Status } \\
\text { or } \\
\text { Ticket \# }\end{array}$ \\
\end{tabular} & $\begin{array}{c}\text { Frequenc } \\
y(20 \%)\end{array}$ & $\begin{array}{c}\text { Importance } \\
\text { of comment } \\
(80 \%)\end{array}$ & \begin{tabular}{|c|} 
Sum \\
$(100 \%)$
\end{tabular} & $\begin{array}{c}\text { Ease of } \\
\text { Implementatio } \\
\mathrm{n}(30 \%)\end{array}$ & $\begin{array}{c}\text { Duration } \\
(40 \%)\end{array}$ & $\begin{array}{c}\text { Risk/ } \\
\text { Complicatio } \\
\mathrm{n}(30 \%)\end{array}$ & \begin{tabular}{|} 
Sum \\
$(100 \%)$
\end{tabular} \mid & \begin{tabular}{|c|} 
Short Term \\
$(40 \%$ \\
Importance) \\
$(60 \%$ Feasibility) \\
\end{tabular} & $\begin{array}{c}\text { Long Term } \\
(85 \% \\
\text { Importance) } \\
\text { (15\% Feasibility) }\end{array}$ \\
\hline M Myer & MM306 & Internal & Content & \begin{tabular}{|l|} 
Recessed Non- \\
Planar Lensed \\
Lumianire with \\
High BF \\
Ballasts (Office)
\end{tabular} & $9^{\prime}$ & PO & & Approach/Concept & $\begin{array}{l}\text { "The selected luminaires } \\
\text { distribute" - how many } \\
\text { luminaires are there? In the } \\
\text { bullet above it is singular. In } \\
\text { the overhead it is singular. } \\
\text { In the perspective, there } \\
\text { are multiple luminaires }\end{array}$ & Images & 13 & 3 & 4 & 3.8 & 4 & 4 & 5 & 4.3 & 4.0 & 3.9 \\
\hline M Myer & MM367 & Internal & Content & \begin{tabular}{|l} 
Recessed Non- \\
Planar Lensed \\
Lumianire with \\
low BF Ballasts \\
(Office)
\end{tabular} & 9 & PO & & Approach/Concept & $\begin{array}{l}\text { Shouldn't the articulated } \\
\text { luminaire be in the } \\
\text { perspective diagram? }\end{array}$ & Images & 13 & 3 & 4 & 3.8 & 4 & 4 & 5 & 4.3 & 4.0 & 3.9 \\
\hline M Myer & MM200 & Internal & \begin{tabular}{|l|} 
Appearance| \\
Aesthetics
\end{tabular} & $\begin{array}{l}\text { Add Corridors } \\
\text { Space/5' wide }\end{array}$ & & & corridors & Graphics & \begin{tabular}{|l|} 
\\
The corridor images look \\
the same. I see luminaires \\
running down the center of \\
the hallway
\end{tabular} & Images & 13 & 3 & 4 & 3.8 & 4 & 4 & 5 & 4.3 & 4.0 & 3.9 \\
\hline M Myer & MM604 & Internal & \begin{tabular}{|l|} 
Appearance/ \\
Aesthetics
\end{tabular} & $\begin{array}{l}\text { Perimeter } \\
\text { Recessed } \\
\text { Linear } \\
\text { Fluorescent }\end{array}$ & & & & \begin{tabular}{|l} 
Perspective \\
Diagram
\end{tabular} & \begin{tabular}{|l|} 
\\
The fixtures are cuttirng \\
right through ceiling tile \\
support structure. Can this \\
image be redwarn?
\end{tabular} & Images & 13 & 3 & 4 & 3.8 & 4 & 4 & 5 & 4.3 & 4.0 & 3.9 \\
\hline M Myer & MM581 & Internal & Content & \begin{tabular}{|l|} 
Perimter \\
Recessed CFLs
\end{tabular} & & & & \begin{tabular}{|l|} 
Perspective \\
Diagram
\end{tabular} & $\begin{array}{l}\text { The fixtures do not look } \\
\text { round or the right size }\end{array}$ & Images & 13 & 3 & 4 & 3.8 & 4 & 4 & 5 & 4.3 & 4.0 & 3.9 \\
\hline M Myer & MM203 & Internal & \begin{tabular}{|l|}
$\begin{array}{l}\text { Appearancel } \\
\text { Aesthetics }\end{array}$ \\
A
\end{tabular} & \begin{tabular}{|l|} 
Recessed 1'x4' \\
Non-planar \\
lensed \\
luminaires
\end{tabular} & & & corridors & \begin{tabular}{|l|} 
Perspective \\
\end{tabular} & \begin{tabular}{|l|} 
The perspectives are the \\
same for the non-planar \\
and the asymmetric \\
vignettes, yet the overhead \\
plans are slightly different \\
because the luminaires \\
shift about the center of the \\
hallway.
\end{tabular} & Images & 13 & 3 & 4 & 3.8 & 4 & 4 & 5 & 4.3 & 4.0 & 3.9 \\
\hline
\end{tabular}




\begin{tabular}{|c|c|c|c|c|c|c|c|c|c|c|c|c|c|c|c|c|c|c|c|c|}
\hline $\begin{array}{l}\text { Commenter } \\
\text { ID \# }\end{array}$ & \begin{tabular}{|l|} 
Comment \\
Reference \\
$\#$
\end{tabular} & $\begin{array}{l}\text { Commenter } \\
\text { Category }\end{array}$ & $\begin{array}{l}\text { Comment } \\
\text { Type }\end{array}$ & Space Type & \begin{tabular}{|l|} 
Ceiling \\
Height/ \\
Total Area
\end{tabular} & \begin{tabular}{|l|}
$\#$ \\
workstations/ \\
partition \\
height (open \\
office only) \\
\end{tabular} & $\left|\begin{array}{l}\text { Daylight } \\
\text { Input (if } \\
\text { applicable) }\end{array}\right|$ & $\begin{array}{l}\text { Reference webpage } \\
\text { or other location } \\
\text { (see } \\
\text { "Reference.doc") }\end{array}$ & $\begin{array}{l}\text { Comment Description: } \\
\text { Issues, Suggestions \& } \\
\text { Requests }\end{array}$ & Action/Solution $\left[\begin{array}{l}S \\
\text { or } \\
\mathrm{Ti}\end{array}\right.$ & $\begin{array}{l}\text { Status } \\
\text { or } \\
\text { Ticket \# }\end{array}$ & $\begin{array}{c}\text { Frequenc } \\
\mathrm{y}(20 \%)\end{array}$ & $\begin{array}{c}\text { Importance } \\
\text { of comment } \\
(80 \%)\end{array}$ & $\begin{array}{c}\text { Sum } \\
(100 \%)\end{array}$ & $\begin{array}{c}\text { Ease of } \\
\text { Implementatio } \\
\mathrm{n}(30 \%)\end{array}$ & $\begin{array}{c}\text { Duration } \\
(40 \%)\end{array}$ & $\begin{array}{c}\text { Risk/ } \\
\text { Complicatio } \\
\mathrm{n}(30 \%)\end{array}$ & $\begin{array}{c}\text { Sum } \\
(100 \%)\end{array}$ & $\begin{array}{c}\text { Short Term } \\
(40 \% \\
\text { Importance) } \\
\text { (60\% Feasibility) } \\
\end{array}$ & $\begin{array}{c}\text { Long Term } \\
(85 \% \\
\text { Importance) } \\
\text { (15\% Feasibility) }\end{array}$ \\
\hline M Myer & 4 MM328 & $\mid$ Internal & Content & \begin{tabular}{|l|l} 
Suspended & 9 \\
Direct/Indirect \\
Luminaires with \\
low BF ballasts
\end{tabular} & 9 & PO & & Graphics & \begin{tabular}{|l|} 
There are three luminaires \\
in the perspective diagram \\
and only two in the \\
overhead plan
\end{tabular} & Images & 13 & 3 & 4 & 3.8 & 4 & 4 & 5 & 4.3 & 4.0 & 3.9 \\
\hline M Myer & MM456 & Internal & \begin{tabular}{|l|}
$\begin{array}{l}\text { Appearancel } \\
\text { Aesthetics }\end{array}$ \\
\end{tabular} & $\begin{array}{l}\text { Recessed Non- } \\
\text { Planar on wider } \\
\text { centers }\end{array}$ & $9.6 / 5000$ & $40 / 48$ & & Overhead plan & $\begin{array}{l}\text { This image looks the same } \\
\text { as the other recessed non- } \\
\text { planar image. Are you sure } \\
\text { that the spacing is } \\
\text { different? }\end{array}$ & Images & 13 & 3 & 4 & 3.8 & 4 & 4 & 5 & 4.3 & 4.0 & 3.9 \\
\hline M Myer & \begin{tabular}{|l|l|l} 
MM558 &
\end{tabular} & Internal & Content & $\begin{array}{l}\text { Suspended } 8^{\prime} \\
\text { multi-lamp } \\
(0.69 \text { W/sf })\end{array}$ & $10 / 5000$ & $50 / 48$ & & Approach/Concept & $\begin{array}{l}\text { This statement does not } \\
\text { seem to jive with the } \\
\text { graphics "The lighting } \\
\text { system has an orderly } \\
\text { appearance but spacing } \\
\text { may vary. Luminaires are } \\
\text { spaced more closely over } \\
\text { work areas." }\end{array}$ & Images & 13 & 3 & 4 & 3.8 & 4 & 4 & 5 & 4.3 & 4.0 & 3.9 \\
\hline M Myer & MM113 & Internal & Content & \begin{tabular}{|l|} 
Recessed Slot \\
Luminaires \\
Parallel to Desk \\
with \\
Contiunous \\
Linear Wall- \\
washers (office)
\end{tabular} & & & & Luminaires C & $\begin{array}{l}\text { Is the distribution icon for } \\
\text { this luminaire correct? }\end{array}$ & Images & 13 & 3 & 4 & 3.8 & 4 & 4 & 5 & 4.3 & 4.0 & 3.9 \\
\hline N Miller & NM23 & Internal & & & & & & & $\begin{array}{l}\text { The fixtures are } \\
\text { asymmetrical, but the } \\
\text { sketched perspective } \\
\text { shows both walls equally } \\
\text { lighted. }\end{array}$ & Images & 13 & 3 & 4 & 3.8 & 4 & 4 & 5 & 4.3 & 4.0 & 3.9 \\
\hline M Myer & MM83 & Internal & Content & \begin{tabular}{|l|} 
Recessed Slot \\
Lumianirs \\
parallel to desk \\
with compact \\
fluorescent wall \\
washers (office)
\end{tabular} & & & & Strategy/luminaires & \begin{tabular}{|l|} 
\\
This says "C" here yet the \\
box to the right does not \\
show luminaire type C.I \\
would also show luminaire \\
type $C$ in the perspective \\
and possibly in the vignette
\end{tabular} & Images & 23 & 2 & 5 & 4.4 & 4 & 4 & 4 & 4 & 4.2 & 4.3 \\
\hline N Miller & NM24 & Internal & & & & & & & $\begin{array}{l}\text { What are the six white dots } \\
\text { in the overhead plan? }\end{array}$ & Images & 13 & 2 & 5 & 4.4 & 4 & 4 & 5 & 4.3 & 4.4 & 4.4 \\
\hline
\end{tabular}


Appendix E: Master Comments List

\begin{tabular}{|c|c|c|c|c|c|c|c|c|c|c|c|c|c|c|c|c|c|c|c|c|}
\hline $\begin{array}{l}\text { Commenter } \\
\text { ID \# }\end{array}$ & \begin{tabular}{|l|} 
Comment \\
Reference \\
$\#$
\end{tabular} & $\begin{array}{l}\text { Commenter } \\
\text { Category }\end{array}$ & $\begin{array}{l}\text { Comment } \\
\text { Type }\end{array}$ & Space Type & \begin{tabular}{|l|} 
Ceiling \\
Height/ \\
Total Area
\end{tabular} & \begin{tabular}{|l|}
$\#$ \\
workstations/ \\
partition \\
height (open \\
office only)
\end{tabular} & \begin{tabular}{|l} 
Daylight \\
Input (if \\
applicable)
\end{tabular} & \begin{tabular}{|l|} 
Reference webpage \\
or other location \\
(see \\
"Reference.doc")
\end{tabular} & $\begin{array}{l}\text { Comment Description: } \\
\text { Issues, Suggestions \& } \\
\text { Requests }\end{array}$ & Action/Solution & \begin{tabular}{|l|} 
Status \\
or \\
Ticket \#
\end{tabular} & $\begin{array}{c}\text { Frequenc } \\
\mathrm{y}(20 \%)\end{array}$ & $\begin{array}{c}\text { Importance } \\
\text { of comment } \\
(80 \%)\end{array}$ & $\begin{array}{c}\text { Sum } \\
(100 \%)\end{array}$ & $\begin{array}{c}\text { Ease of } \\
\text { Implementatio } \\
\mathrm{n}(30 \%)\end{array}$ & $\begin{array}{c}\text { Duration } \\
(40 \%)\end{array}$ & $\begin{array}{c}\text { Risk/ } \\
\text { Complicatio } \\
\mathrm{n}(30 \%)\end{array}$ & $\begin{array}{c}\text { Sum } \\
(100 \%)\end{array}$ & \begin{tabular}{|c|} 
Short Term \\
$(40 \%$ \\
Importance) \\
(60\% Feasibility)
\end{tabular} & \begin{tabular}{|c|} 
Long Term \\
(85\% \\
Importance) \\
(15\% Feasibility)
\end{tabular} \\
\hline$\overline{\text { A Cortese }}$ & AC14 & Architect & Content & & & & & & $\begin{array}{l}\text { Recessed non planar on } \\
\text { wider centers shows the } \\
\text { exact same lighting layout } \\
\text { as the regular Recessed } \\
\text { non planar lighting } \\
\text { scenario. I'm assuming } \\
\text { thought that they should } \\
\text { have different plans since } \\
\text { one means fewer fixtures } \\
\text { (and lower LPD). This is } \\
\text { where the visuals might } \\
\text { make a difference. } \\
\end{array}$ & Images & 13 & 3 & 5 & 4.6 & 4 & 4 & 5 & 4.3 & 4.5 & 4.6 \\
\hline \begin{tabular}{|l|} 
C DiLouie \\
\end{tabular} & CD29 & Core Team & Content & & & & & & $\begin{array}{l}\text { Check all control guidance } \\
\text { drawings to ensure you are } \\
\text { using the latest drawings. I } \\
\text { noticed on some of the } \\
\text { drawings that the legend is } \\
\text { outdated. The legend for } \\
\text { each drawing should look } \\
\text { like the legend for the } \\
\text { private office drawing } \\
\text { (dimming and vacancy } \\
\text { sensing option), but without } \\
\text { the numbers on the left. }\end{array}$ & Images & 13 & 3 & 5 & 4.6 & 4 & 4 & 5 & 4.3 & 4.5 & 4.6 \\
\hline C Dilouie & CD28 & Core Team & Content & & & & & & \begin{tabular}{|l|} 
The text on the control \\
guidance figures should be \\
larger, more legible. \\
Colored lines should be \\
stronger. Switch, etc. \\
symbols larger.
\end{tabular} & Images & 13 & 3 & 5 & 4.6 & 4 & 4 & 5 & 4.3 & 4.5 & 4.6 \\
\hline \begin{tabular}{|l|} 
C DiLouie \\
\end{tabular} & CD36 & Core Team & Content & & & & & & $\begin{array}{l}\text { Some control guidance } \\
\text { drawings are also } \\
\text { worrisome, particularly } \\
\text { where we are showing } \\
\text { multiple sensor locations. } \\
\text { The solution I had } \\
\text { proposed was to show a } \\
\text { small inset and show } \\
\text { multiple locations for say a } \\
\text { vacancy sensor in a } \\
\text { conference room-one } \\
\text { over the door, one in the } \\
\text { middle of the room, etc. } \\
\text { Same with photosensors, } \\
\text { one by the window, one } \\
\text { over the task area. Instead, } \\
\text { the inset is not used, and it } \\
\text { looks like there are three } \\
\text { vacancy sensors and two } \\
\text { photosensors in the } \\
\text { meeting rooms. Plus the } \\
\text { photosensor should not be } \\
\text { a \$ symbol but instead an } \\
\text { asterisk. Please review all } \\
\text { the control guidance } \\
\text { drawings and ensure they } \\
\text { correctly match the last } \\
\text { drawings submitted. }\end{array}$ & Images & 13 & 3 & 5 & 4.6 & 4 & 4 & 5 & 4.3 & 4.5 & 4.6 \\
\hline
\end{tabular}


Appendix E: Master Comments List

\begin{tabular}{|c|c|c|c|c|c|c|c|c|c|c|c|c|c|c|c|c|c|c|c|c|}
\hline $\begin{array}{l}\text { Commenter } \\
\text { ID \# }\end{array}$ & \begin{tabular}{|l|} 
Comment \\
Reference \\
$\#$
\end{tabular} & $\begin{array}{l}\text { Commenter } \\
\text { Category }\end{array}$ & \begin{tabular}{|l} 
Comment \\
Type
\end{tabular} & Space Type & \begin{tabular}{|l|} 
Ceiling \\
Height/ \\
Total Area
\end{tabular} & \begin{tabular}{|l|}
$\#$ \\
workstations/ \\
partition \\
height (open \\
office only) \\
\end{tabular} & \begin{tabular}{|l|} 
Daylight \\
Input (if \\
applicable)
\end{tabular} & 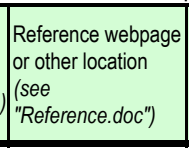 & $\begin{array}{l}\text { Comment Description: } \\
\text { Issues, Suggestions \& } \\
\text { Requests }\end{array}$ & Action/Sc & \begin{tabular}{|l|} 
Status \\
or \\
Ticket \#
\end{tabular} & $\begin{array}{c}\text { Frequenc } \\
\text { y (20\%) }\end{array}$ & $\begin{array}{c}\text { Importance } \\
\text { of comment } \\
(80 \%)\end{array}$ & \begin{tabular}{|c|} 
Sum \\
$(100 \%)$
\end{tabular} & $\begin{array}{c}\text { Ease of } \\
\text { Implementatio } \\
\mathrm{n}(30 \%)\end{array}$ & \begin{tabular}{|c} 
Duration \\
$(40 \%)$
\end{tabular} & $\begin{array}{c}\text { Risk/ } \\
\text { Complicatio } \\
\mathrm{n}(30 \%)\end{array}$ & \begin{tabular}{|c|} 
Sum \\
$(100 \%)$
\end{tabular} & 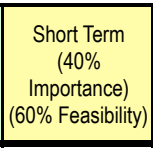 & $\begin{array}{c}\text { Long Term } \\
(85 \% \\
\text { Importance) } \\
\text { (15\% Feasibility) } \\
\end{array}$ \\
\hline C Dilouie & CD34 & Core Team & Content & & & & & & \begin{tabular}{|l|} 
The cross section \\
drawings are a bit \\
worrisome. I noticed a few \\
cases where "occupancy \\
sensor" was used instead \\
of "vacancy sensor," which \\
should be fixed for \\
consistency. As another \\
example, in the private \\
office drawing, it should be \\
photosensor, not \\
photosensors, the manual \\
dimming allows user to \\
select light levels, and there \\
is one vacancy sensor, not \\
plural, and it switches both \\
control zones off when the \\
space is unoccupied, not \\
each... If you would like to \\
send me all the cross \\
section drawings in a \\
separate email, I would be \\
happy to review them.
\end{tabular} & Images & 13 & 3 & 5 & 4.6 & 4 & 4 & 5 & 4.3 & 4.5 & 4.6 \\
\hline D Maniccia & DM9 & Controls & & & & & & & $\begin{array}{l}\text { In plan view the location for } \\
\text { the sensor looks like it's } \\
\text { behind the door. }\end{array}$ & Images & 13 & 3 & 5 & 4.6 & 4 & 4 & 5 & 4.3 & 4.5 & 4.6 \\
\hline D Maniccia & DM8 & Controls & & & & & & \begin{tabular}{|l|} 
Private Office \\
Controls Template \\
22: Vacancy \\
Sensing, Multilevel \\
Switching
\end{tabular} & \begin{tabular}{|l|} 
Plan diagram show 3 dots. \\
Perhaps instead you could \\
show A, B, and C, to \\
indicate that it's the same \\
thing in 3 possible \\
locations?
\end{tabular} & Images & 13 & 3 & 5 & 4.6 & 4 & 4 & 5 & 4.3 & 4.5 & 4.6 \\
\hline HMcKay & HM35 & Designer & Content & & & & & & $\begin{array}{l}\text { Colored zones should be } \\
\text { modified. Green zone } \\
\text { should move left and } \\
\text { extend to screen wall. } \\
\text { There should be an } \\
\text { additional } \\
\text { pink zone adjacent to the } \\
\text { right wall. }\end{array}$ & Images & 13 & 3 & 5 & 4.6 & 4 & 4 & 5 & 4.3 & 4.5 & 4.6 \\
\hline HMcKay & HM21 & Designer & Content & & & & & & $\begin{array}{l}\text { Graphic outlines are too } \\
\text { thin to clearly read the } \\
\text { color. }\end{array}$ & Images & 13 & 3 & 5 & 4.6 & 4 & 4 & 5 & 4.3 & 4.5 & 4.6 \\
\hline HMcKay & HM41 & Designer & Content & & & & & & \begin{tabular}{|l|} 
Plan view: corridor outside \\
of elevator lobby should not \\
be dead-end. Remove wall \\
on the right to give \\
impression of continuous \\
corridor (and proper \\
egress). \\
\end{tabular} & Images & 13 & 3 & 5 & 4.6 & 4 & 4 & 5 & 4.3 & 4.5 & 4.6 \\
\hline HMcKay & HM3O & Designer & Content & & & & & & $\begin{array}{l}\text { V1 - Described as Linear } \\
\text { WW, but wrong plan shows } \\
\text { CFL. Expanded plan has } \\
\text { wrong fixture types. }\end{array}$ & Images & 13 & 3 & 5 & 4.6 & 4 & 4 & 5 & 4.3 & 4.5 & 4.6 \\
\hline
\end{tabular}


Appendix E: Master Comments List

\begin{tabular}{|c|c|c|c|c|c|c|c|c|c|c|c|c|c|c|c|c|c|c|c|c|}
\hline $\begin{array}{l}\text { Commenter } \\
\text { ID \# }\end{array}$ & $\begin{array}{l}\text { Comment } \\
\text { Reference } \\
\#\end{array}$ & $\begin{array}{l}\text { Commenter } \\
\text { Category }\end{array}$ & $\begin{array}{l}\text { Comment } \\
\text { Type }\end{array}$ & Space Type & \begin{tabular}{|l|} 
Ceiling \\
Height/ \\
Total Area
\end{tabular} & \begin{tabular}{|l|}
$\#$ \\
workstations/ \\
partition \\
height (open \\
office only) \\
\end{tabular} & $\begin{array}{l}\text { Daylight } \\
\text { Input (if } \\
\text { applicable) }\end{array}$ & \begin{tabular}{|l|} 
Reference webpage \\
or other location \\
(see \\
"Reference.doc")
\end{tabular} & $\begin{array}{l}\text { Comment Description: } \\
\text { Issues, Suggestions \& } \\
\text { Requests }\end{array}$ & Action/Solution $/$ S & \begin{tabular}{|l|} 
Status \\
or \\
Ticket \#
\end{tabular} & \begin{tabular}{|c|} 
Frequenc \\
$\mathrm{y}(20 \%)$
\end{tabular} & $\begin{array}{c}\text { Importance } \\
\text { of comment } \\
(80 \%)\end{array}$ & \begin{tabular}{|c} 
Sum \\
$(100 \%)$
\end{tabular} & $\begin{array}{c}\text { Ease of } \\
\text { Implementatio } \\
\mathrm{n}(30 \%)\end{array}$ & $\begin{array}{c}\text { Duration } \\
(40 \%)\end{array}$ & $\begin{array}{c}\text { Risk/ } \\
\text { Complicatio } \\
\mathrm{n}(30 \%)\end{array}$ & \begin{tabular}{|} 
Sum \\
$(100 \%)$
\end{tabular} & \begin{tabular}{|c|} 
Short Term \\
$(40 \%$ \\
Importance) \\
(60\% Feasibility)
\end{tabular} & $\begin{array}{c}\text { Long Term } \\
\text { (85\% } \\
\text { Importance) } \\
\text { (15\% Feasibility) } \\
\end{array}$ \\
\hline HMcKay & HM27 & Designer & Content & & & & & & $\begin{array}{l}\text { V1- Description is for 12' } \\
\text { and 8' fixtures, but plan \& } \\
\text { perspectives show 8' and } 4 \text { ' } \\
\text { fixtures. Type B and C are } \\
\text { not called out } \\
\text { on plan or perspective. }\end{array}$ & Images & 13 & 3 & 5 & 4.6 & 4 & 4 & 5 & 4.3 & 4.5 & 4.6 \\
\hline HMcKay & HM31 & Designer & Content & & & & & & $\begin{array}{l}\text { V2 - Expanded plan - } \\
\text { wrong fixture types. }\end{array}$ & Images & 13 & 3 & 5 & 4.6 & 4 & 4 & 5 & 4.3 & 4.5 & 4.6 \\
\hline HMcKay & HM29 & Designer & Content & & & & & & \begin{tabular}{|l|} 
V3 - Type B and C are not \\
called out on plan or \\
perspective.
\end{tabular} & Images & 13 & 3 & 5 & 4.6 & 4 & 4 & 5 & 4.3 & 4.5 & 4.6 \\
\hline HMcKay & HM45 & Designer & \begin{tabular}{|l|} 
Content \\
\end{tabular} & & & & & & $\begin{array}{l}\text { V3 - WW do not show up } \\
\text { in small plan. Fixture types } \\
\text { mislabeled in plans and } \\
\text { perspective. Type B is wall } \\
\text { wash, not } \\
\text { downlight. }\end{array}$ & Images & 13 & 3 & 5 & 4.6 & 4 & 4 & 5 & 4.3 & 4.5 & 4.6 \\
\hline HMcKay & HM33 & Designer & Content & & & & & & \begin{tabular}{|l|} 
V4 - Expanded plan does \\
not match small plan, and \\
has wrong fixture types. \\
Description is for 4' fixtures \\
but plans and \\
perspective show longer \\
fixtures. \\
\end{tabular} & Images & 13 & 3 & 5 & 4.6 & 4 & 4 & 5 & 4.3 & 4.5 & 4.6 \\
\hline HMcKay & HM43 & Designer & Content & & & & & & $\begin{array}{l}\text { V1- Linear slot. No small } \\
\text { plan, only enlarged plan. }\end{array}$ & Images & 13 & 3 & 5 & 4.6 & 4 & 4 & 5 & 4.3 & 4.5 & 4.6 \\
\hline HMcKay & HM38 & Designer & Content & & & & & & $\begin{array}{l}\text { vignette } 1 \text { - Section: } \\
\text { Shows daylight at the long } \\
\text { end of the room. Should } \\
\text { each lamp be shown in two } \\
\text { colors, to indicate two-level } \\
\text { switching? }\end{array}$ & Images & 13 & 3 & 5 & 4.6 & 4 & 4 & 5 & 4.3 & 4.5 & 4.6 \\
\hline HMcKay & HM47 & Designer & Content & & & & & & \begin{tabular}{|l|} 
Reception controls \\
template 1 for Vignette 4. \\
Section also shows \\
fixture on left with down \\
distribution, not wall wash \\
distribution. This could be a \\
separate green zone and \\
turned off after hours. Plan \\
should be same as for \\
Vignettes 1 and 3 . \\
\end{tabular} & Images & 13 & 3 & 5 & 4.6 & 4 & 4 & 5 & 4.3 & 4.5 & 4.6 \\
\hline \begin{tabular}{|l}
$J$ Beutell \\
\end{tabular} & JB55 & Owner & & & & & & \begin{tabular}{|l|} 
Suspended 8' Multi- \\
Lamp Direct//ndirect \\
Luminaires (Office)
\end{tabular} & $\begin{array}{l}\text { Labels aren't matching the } \\
\text { list. }\end{array}$ & Images & 13 & 3 & 5 & 4.6 & 4 & 4 & 5 & 4.3 & 4.5 & 4.6 \\
\hline J Yorgey & JY31 & Manufacturer & \begin{tabular}{|l|} 
Appearance \\
Aesthetics
\end{tabular} & & & & & \begin{tabular}{|l|} 
Office design \\
vinettes -open plan \\
perimeter spaces
\end{tabular} & \begin{tabular}{|l|} 
Not clear on drawing that \\
shows space type Open \\
plan perimeter shows both \\
interior wall and window \\
wall - this is confusing - \\
should just do interior wall \\
and leave daylighting in \\
"open Plan"
\end{tabular} & Images & 13 & 3 & 5 & 4.6 & 4 & 4 & 5 & 4.3 & 4.5 & 4.6 \\
\hline
\end{tabular}




\begin{tabular}{|c|c|c|c|c|c|c|c|c|c|c|c|c|c|c|c|c|c|c|c|c|}
\hline $\begin{array}{l}\text { Commenter } \\
\text { ID \# }\end{array}$ & $\begin{array}{l}\text { Comment } \\
\text { Reference } \\
\#\end{array}$ & $\begin{array}{l}\text { Commenter } \\
\text { Category }\end{array}$ & \begin{tabular}{|l} 
Comment \\
Type
\end{tabular} & Space Type & $\begin{array}{l}\text { Ceiling } \\
\text { Height/ } \\
\text { Total Area }\end{array}$ & $\begin{array}{l}\# \\
\text { workstations/ } \\
\text { partition } \\
\text { height (open } \\
\text { office only) } \\
\end{array}$ & $\mid \begin{array}{l}\text { Daylight } \\
\text { Input (if } \\
\text { applicable) }\end{array}$ & $\begin{array}{l}\text { Reference webpage } \\
\text { or other location } \\
\text { (see } \\
\text { "Reference.doc") }\end{array}$ & \begin{tabular}{|l|} 
Comment Description: \\
Issues, Suggestions \& \\
Requests
\end{tabular} & Action/Solution & $\begin{array}{l}\text { Status } \\
\text { or } \\
\text { Ticket \# }\end{array}$ & $\begin{array}{c}\text { Frequenc } \\
\text { y (20\%) }\end{array}$ & $\begin{array}{c}\text { Importance } \\
\text { of comment } \\
(80 \%)\end{array}$ & \begin{tabular}{|c|} 
Sum \\
$(100 \%)$
\end{tabular} & $\begin{array}{c}\text { Ease of } \\
\text { Implementatio } \\
\mathrm{n}(30 \%)\end{array}$ & $\begin{array}{c}\text { Duration } \\
(40 \%)\end{array}$ & $\begin{array}{c}\text { Risk/ } \\
\text { Complicatio } \\
\mathrm{n}(30 \%)\end{array}$ & \begin{tabular}{|c|} 
Sum \\
$(100 \%)$
\end{tabular} & $\begin{array}{c}\begin{array}{c}\text { Short Term } \\
(40 \% \\
\text { Importance) } \\
(60 \% \text { Feasibility })\end{array} \\
\end{array}$ & $\begin{array}{c}\text { Long Term } \\
\text { (85\% } \\
\text { Importance) } \\
\text { (15\% Feasibility) } \\
\end{array}$ \\
\hline LDavis & LD7 & Designer & & & & & & $\begin{array}{l}\text { open plan, } \\
\text { suspended 12" } \\
\text { single lamp d/l } \\
\text { luminaires }\end{array}$ & \begin{tabular}{|l|} 
there are more luminaire \\
types in the list than show \\
up on the graphics. It \\
appears that the technical \\
information is right, but the \\
graphics are missing labels.
\end{tabular} & Images & 13 & 3 & 5 & 4.6 & 4 & 4 & 5 & 4.3 & 4.5 & 4.6 \\
\hline M Myer & MM492 & Internal & Content & \begin{tabular}{|l|} 
Recessed Non- \\
Planar (LPD \\
0.64 W/sf) \\
\end{tabular} & $8 / 5000$ & $30 / 48$ & & LPD & \begin{tabular}{|l|} 
Why is the LPD here 0.64 \\
for 5,000 sq ft with $48 "$ \\
paritions and 30 work \\
stations and for another \\
design it is 0.56 for 5,000 \\
sq ft with $48 "$ paritions and \\
40 work stations. The \\
overhead plans for this \\
LPD and another LPD \\
appears to be the same \\
plan.
\end{tabular} & Images & 13 & 3 & 5 & 4.6 & 4 & 4 & 5 & 4.3 & 4.5 & 4.6 \\
\hline M Myer & MM270 & Internal & \begin{tabular}{|l|} 
Appearancel \\
Aesthetics
\end{tabular} & $\begin{array}{l}\text { Recessed 4' } \\
\text { Asymmetric } \\
\text { wall-wash } \\
\text { luminaires }\end{array}$ & & & corridors & Overhead diagram & $\begin{array}{l}\text { In the overhead diagram, } \\
\text { which way is the light being } \\
\text { directed? Is the "A" fixture } \\
\text { lighting the open air? Are } \\
\text { the fixtures meant to be } 3^{\prime} \\
\text { away from the wall that has } \\
\text { the chairs for the } \\
\text { conference room in it? }\end{array}$ & Images & 13 & 3 & 5 & 4.6 & 4 & 4 & 5 & 4.3 & 4.5 & 4.6 \\
\hline M Myer & MM415 & Internal & \begin{tabular}{|l|} 
Appearance| \\
Aesthetics
\end{tabular} & $\begin{array}{l}\text { Suspended 12' } \\
\text { single-lamp }\end{array}$ & $9.6 / 5000$ & $40 / 48$ & & Images & \begin{tabular}{|l|} 
Luminaire box shows both \\
"A" and "B" an 8 ' and 4 ' \\
luminaire respectively. \\
Neither of the graphics call \\
out luminaire types $A$ or $B$ \\
\end{tabular} & Images & 13 & 3 & 5 & 4.6 & 4 & 4 & 5 & 4.3 & 4.5 & 4.6 \\
\hline M Myer & MM540 & Internal & Content & $\begin{array}{l}\text { Suspended 8' } \\
\text { multi-lamp } \\
\text { (0.69 W/sf) }\end{array}$ & $10 / 5000$ & $50 / 48$ & & Overhead plan & \begin{tabular}{|l|} 
Luminaires box calls out \\
both "A" and "B" luminaires, \\
but the overhead plan does \\
not show Luminaire B
\end{tabular} & Images & 13 & 3 & 5 & 4.6 & 4 & 4 & 5 & 4.3 & 4.5 & 4.6 \\
\hline M Myer & MM355 & Internal & \begin{tabular}{|l|} 
Appearancel \\
Aesthetics
\end{tabular} & \begin{tabular}{|l|} 
Recessed Non- \\
Planar Lensed \\
Lumianire with \\
low BF Ballasts \\
(Office)
\end{tabular} & $9^{\prime}$ & PO & & \begin{tabular}{|l|} 
Perspective \\
Diagram and \\
Overhead Plan
\end{tabular} & $\begin{array}{l}\text { Perspective shows two } \\
\text { luminaires and the } \\
\text { overhead plan shows } 1 \\
\text { luminaire }\end{array}$ & Images & 13 & 3 & 5 & 4.6 & 4 & 4 & 5 & 4.3 & 4.5 & 4.6 \\
\hline M Myer & \begin{tabular}{|l|} 
MM14 \\
\end{tabular} & Internal & Content & \begin{tabular}{|l|} 
Recessed Non- \\
Planar Lensed \\
Luminaires with \\
low Ballast \\
Factor and \\
Recessed \\
Compact \\
Fluorescent \\
Lensed Wall- \\
washers \\
(Office) \\
\end{tabular} & \begin{tabular}{|l|} 
Conferenc \\
e room
\end{tabular} & & & $\begin{array}{l}\text { Strategy/Luminaire } \\
\text { B }\end{array}$ & \begin{tabular}{|} 
The "B" luminaire text does \\
not match either of the \\
graphics for Overhead or \\
Perspective plans
\end{tabular} & Images & 13 & 3 & 5 & 4.6 & 4 & 4 & 5 & 4.3 & 4.5 & 4.6 \\
\hline M Myer & MM605 & Internal & \begin{tabular}{|l|} 
Appearancel \\
Aesthetics \\
\end{tabular} & $\begin{array}{l}\text { Perimeter } \\
\text { Recessed } \\
\text { Linear } \\
\text { Fluorescent } \\
\end{array}$ & & & & Overhead plan & \begin{tabular}{|l|} 
The diagram calls out \\
fixture B. The luminaire box \\
says luminaire "A"
\end{tabular} & Images & 13 & 3 & 5 & 4.6 & 4 & 4 & 5 & 4.3 & 4.5 & 4.6 \\
\hline M Myer & MM578 & |Internal & Content & $\mid \begin{array}{l}\text { Perimter } \\
\text { Recessed CFLs }\end{array}$ & & & & Overhead plan & \begin{tabular}{|l|l|}
$\begin{array}{l}\text { The fixtures really do not } \\
\text { stand out. Anything that we } \\
\text { can do? }\end{array}$ & \\
con
\end{tabular} & Images & 13 & 3 & 5 & 4.6 & 4 & 4 & 5 & 4.3 & 4.5 & 4.6 \\
\hline
\end{tabular}




\begin{tabular}{|c|c|c|c|c|c|c|c|c|c|c|c|c|c|c|c|c|c|c|c|c|}
\hline $\begin{array}{l}\text { Commenter } \\
\text { ID \# }\end{array}$ & \begin{tabular}{|l|} 
Comment \\
Reference \\
$\#$
\end{tabular} & $\begin{array}{l}\text { Commenter } \\
\text { Category }\end{array}$ & $\begin{array}{l}\text { Comment } \\
\text { Type }\end{array}$ & Space Type & \begin{tabular}{|l|} 
Ceiling \\
Height/ \\
Total Area
\end{tabular} & \begin{tabular}{|l}
$\#$ \\
workstations/ \\
partition \\
height (open \\
office only) \\
\end{tabular} & $\mid$\begin{tabular}{|l|} 
Daylight \\
Input (if \\
applicable)
\end{tabular} & $\begin{array}{l}\text { Reference webpage } \\
\text { or other location } \\
\text { (see } \\
\text { "Reference.doc") }\end{array}$ & $\begin{array}{l}\text { Comment Description: } \\
\text { Issues, Suggestions \& } \\
\text { Requests }\end{array}$ & Action/Solution & \begin{tabular}{|l|} 
Status \\
or \\
Ticket \#
\end{tabular} & $\begin{array}{c}\text { Frequenc } \\
\mathrm{y}(20 \%)\end{array}$ & $\begin{array}{c}\text { Importance } \\
\text { of comment } \\
(80 \%)\end{array}$ & \begin{tabular}{|c|} 
Sum \\
$(100 \%)$
\end{tabular} & $\begin{array}{c}\text { Ease of } \\
\text { Implementatio } \\
\mathrm{n}(30 \%)\end{array}$ & $\begin{array}{c}\text { Duration } \\
(40 \%)\end{array}$ & $\begin{array}{c}\text { Risk/ } \\
\text { Complicatio } \\
\mathrm{n}(30 \%)\end{array}$ & \begin{tabular}{|c|} 
Sum \\
$(100 \%)$
\end{tabular} & \begin{tabular}{|c|} 
Short Term \\
$(40 \%$ \\
Importance) \\
(60\% Feasibility) \\
\end{tabular} & $\begin{array}{c}\text { Long Term } \\
(85 \% \\
\text { Importance) } \\
\text { (15\% Feasibility) }\end{array}$ \\
\hline M Myer & $\overline{M M 3}$ & Internal & $\begin{array}{l}\text { Appearance } \\
\text { Aesthetics }\end{array}$ & $\begin{array}{l}\text { Recessed Non- } \\
\text { Planar Lensed } \\
\text { Luminaires with } \\
\text { low Ballast } \\
\text { Factor and } \\
\text { Recessed } \\
\text { Compact } \\
\text { Fluorescent } \\
\text { Lensed Wall- } \\
\text { washers } \\
\text { (Office) } \\
\end{array}$ & $\begin{array}{l}\text { Conferenc } \\
\text { e room }\end{array}$ & & & Overhead & \begin{tabular}{|l|} 
The overhead shows end- \\
to-end fixtures and might \\
be suspended fixtures. The \\
perspective in contrast \\
shows recessed $2 x 4 \mathrm{~s}$ \\
separated by at least 4 ' of \\
ceiling tile.
\end{tabular} & Images & 13 & 3 & 5 & 4.6 & 4 & 4 & 5 & 4.3 & 4.5 & 4.6 \\
\hline M Myer & MM21 & Internal & \begin{tabular}{|l|} 
Appearance \\
Aesthetics
\end{tabular} & \begin{tabular}{|l|} 
Suspended \\
Direct/lndirect \\
Luminaires, \\
Linear Wall- \\
washers, and a \\
Whiteboard \\
Light \\
\end{tabular} & \begin{tabular}{|l|}
$\begin{array}{l}\text { Conferenc } \\
\text { e room }\end{array}$ \\
\end{tabular} & & & Overhead & $\begin{array}{l}\text { The overhead shows } \\
\text { recessed fixtures and the } \\
\text { perspective shows } \\
\text { suspended fixtures. }\end{array}$ & Images & 13 & 3 & 5 & 4.6 & 4 & 4 & 5 & 4.3 & 4.5 & 4.6 \\
\hline M Myer & MM22 & Internal & \begin{tabular}{|l|} 
Appearance \\
Aesthetics
\end{tabular} & \begin{tabular}{|l|} 
Suspended \\
Direct/lndirect \\
Luminaires, \\
Linear Wall- \\
washers, and a \\
Whiteboard \\
Light \\
\end{tabular} & \begin{tabular}{|l|}
$\begin{array}{l}\text { Conferenc } \\
\text { e room }\end{array}$ \\
\end{tabular} & & & Overhead & $\begin{array}{l}\text { Where is the whiteboard } \\
\text { light fixture? }\end{array}$ & Images & 13 & 3 & 5 & 4.6 & 4 & 4 & 5 & 4.3 & 4.5 & 4.6 \\
\hline M Myer & MM159 & Internal & \begin{tabular}{|l|} 
Appearance \\
Aesthetics
\end{tabular} & \begin{tabular}{|l|} 
Recessed slot \\
luminaires \\
Perpendicular \\
to Desk with \\
Compact \\
Fluorescent \\
Downlights and \\
Wall-washers \\
(Office)
\end{tabular} & & & & \begin{tabular}{|l|} 
Overhead Diagram \\
\end{tabular} & \begin{tabular}{|l|} 
Your overhead and \\
perspective diagrams are \\
wrong. The luminaire box \\
says "A" is the linear slot \\
that is correct. "B" is the \\
CFL wall washer, the \\
graphic shows this as the \\
DL. "C" is the continous \\
wall slot and the grapic \\
shows C as the CFL wall \\
washer. "D" is the CFL \\
downlight and the graphic \\
shows it as the slot.
\end{tabular} & Images & 13 & 3 & 5 & 4.6 & 4 & 4 & 5 & 4.3 & 4.5 & 4.6 \\
\hline M Myer & MM637 & Internal & Content & \begin{tabular}{|l|} 
Controls \\
Strategies: \\
Scheduling, \\
Daylight \\
Harvesting
\end{tabular} & & & & Section Diagram & $\begin{array}{l}\text { Add text about lighting be } \\
\text { turned off via time clock. } \\
\text { We need to reinforce the } \\
\text { term "scheduling". That is } \\
\text { the title of the control } \\
\text { vignette. Also, we should } \\
\text { probably say that it } \\
\text { automatically turns off the } \\
\text { lights when the office } \\
\text { closes. }\end{array}$ & Images & 13 & 3 & 5 & 4.6 & 4 & 4 & 5 & 4.3 & 4.5 & 4.6 \\
\hline N Miller & NM14 & Internal & Content & \begin{tabular}{|l|} 
Office-conf rm \\
\end{tabular} & & & & $\begin{array}{l}\text { Susp dirlind lum's } \\
\text { and rec cfl lensed } \\
\text { WW's }\end{array}$ & $\begin{array}{l}\text { Perspective sketch doesn't } \\
\text { show round CFL } \\
\text { wallwashers, and neither } \\
\text { does overhead plan. }\end{array}$ & Images & 13 & 3 & 5 & 4.6 & 4 & 4 & 5 & 4.3 & 4.5 & 4.6 \\
\hline \begin{tabular}{|l|} 
A Cortese \\
\end{tabular} & AC17 & Architect & \begin{tabular}{|l|} 
Download \\
Materials
\end{tabular} & & & & & & $\begin{array}{l}\text { The Vignette Summaries } \\
\text { and Implementation } \\
\text { Instructions report never } \\
\text { worked. }\end{array}$ & Images & 13 & 4 & 5 & 4.8 & 4 & 4 & 5 & 4.3 & 4.6 & 4.7 \\
\hline
\end{tabular}




\begin{tabular}{|c|c|c|c|c|c|c|c|c|c|c|c|c|c|c|c|c|c|c|c|c|}
\hline $\begin{array}{l}\text { Commenter } \\
\text { ID\# }\end{array}$ & \begin{tabular}{|l|} 
Comment \\
Reference \\
\#
\end{tabular} & $\begin{array}{l}\text { Commenter } \\
\text { Category }\end{array}$ & $\begin{array}{l}\text { Comment } \\
\text { Type }\end{array}$ & Space Type & \begin{tabular}{|l|} 
Ceiling \\
Height/ \\
Total Area
\end{tabular} & \begin{tabular}{|l|}
$\#$ \\
workstations/ \\
partition \\
height (open \\
office only) \\
\end{tabular} & $\left|\begin{array}{l}\text { Daylight } \\
\text { Input (if } \\
\text { applicable) }\end{array}\right|$ & \begin{tabular}{|l|} 
Reference webpage \\
or other location \\
(see \\
"Reference.doc")
\end{tabular} & $\begin{array}{l}\text { Comment Description: } \\
\text { Issues, Suggestions \& } \\
\text { Requests }\end{array}$ & Action/Solution & $\mid \begin{array}{l}\text { Status } \\
\text { or } \\
\text { Ticket \# }\end{array}$ & $\begin{array}{c}\text { Frequenc } \\
\mathrm{y}(20 \%)\end{array}$ & $\begin{array}{c}\text { Importance } \\
\text { of comment } \\
(80 \%)\end{array}$ & $\begin{array}{c}\text { Sum } \\
(100 \%)\end{array}$ & $\begin{array}{c}\text { Ease of } \\
\text { Implementatio } \\
\mathrm{n}(30 \%)\end{array}$ & \begin{tabular}{|c|} 
Duration \\
$(40 \%)$
\end{tabular} & $\begin{array}{c}\text { Risk/ } \\
\text { Complicatio } \\
\mathrm{n}(30 \%)\end{array}$ & \begin{tabular}{|} 
Sum \\
$(100 \%)$
\end{tabular} \mid & $\begin{array}{c}\text { Short Term } \\
(40 \% \\
\text { Importance) } \\
\text { (60\% Feasibility) }\end{array}$ & $\begin{array}{c}\text { Long Term } \\
(85 \% \\
\text { Importance) } \\
\text { (15\% Feasibility) } \\
\end{array}$ \\
\hline A Cortese & AC5 & Architect & \begin{tabular}{|l|} 
Appearancel \\
Aesthetics
\end{tabular} & & & & & & \begin{tabular}{|l|} 
The graphic perspective \\
and plan diagrams are \\
critical for those of us who \\
would rather look at \\
pictures than read. \\
However on some \\
vignettes (specifically \\
covering controls), the plan \\
and sections are \\
unreadable because you \\
can't blow them up big \\
enough. Being able to print \\
them might be helpful. You \\
also might consider \\
increasing the visual appeal \\
of the tool by adding \\
photographs of actual \\
installations.
\end{tabular} & Images & 13 & 4 & 5 & 4.8 & 4 & 4 & 5 & 4.3 & 4.6 & 4.7 \\
\hline C DiLouie & CD15 & Core Team & Content & & & & & & $\begin{array}{l}\text { On all control drawings, } \\
\text { change "flourescent" to } \\
\text { "fluorescent" }\end{array}$ & Images & 13 & 4 & 5 & 4.8 & 4 & 4 & 5 & 4.3 & 4.6 & 4.7 \\
\hline C Dilouie & CD35 & Core Team & Content & & & & & & $\begin{array}{l}\begin{array}{l}\text { On all drawings, make sure } \\
\text { "fluorescent" is spelled } \\
\text { "fluorescent" and not } \\
\text { "flourescent." }\end{array} \\
\end{array}$ & Images & 13 & 4 & 5 & 4.8 & 4 & 4 & 5 & 4.3 & 4.6 & 4.7 \\
\hline C Magee & CM5 & Owner & & & & & & & $\begin{array}{l}\text { Font is too small on } \\
\text { controls plan view. }\end{array}$ & Images & 13 & 4 & 5 & 4.8 & 4 & 4 & 5 & 4.3 & 4.6 & 4.7 \\
\hline D Maniccia & DM7 & Controls & & & & & & & \begin{tabular}{|l} 
Font is too small on \\
controls diagrams (plan \\
view). \\
\end{tabular} & Images & 13 & 4 & 5 & 4.8 & 4 & 4 & 5 & 4.3 & 4.6 & 4.7 \\
\hline HMcKay & HM34 & Designer & Content & & & & & & $\begin{array}{l}\text { Can't read key, even when } \\
\text { expanded. }\end{array}$ & Images & 13 & 4 & 5 & 4.8 & 4 & 4 & 5 & 4.3 & 4.6 & 4.7 \\
\hline HMcKay & HM18 & Designer & Content & & & & & & \begin{tabular}{l|} 
Cannot read text for control \\
diagrams, even enlarged.
\end{tabular} & Images & 13 & 4 & 5 & 4.8 & 4 & 4 & 5 & 4.3 & 4.6 & 4.7 \\
\hline L Davis & LD12 & Designer & & & & & & & $\begin{array}{l}\text { The text is too small on the } \\
\text { controls diagram. }\end{array}$ & Images & 13 & 4 & 5 & 4.8 & 4 & 4 & 5 & 4.3 & 4.6 & 4.7 \\
\hline M Dare & MD10 & Utility & & & & & & & \begin{tabular}{|l|} 
Lettering is too small on the \\
controls plan view. Would \\
also be nicer if the font was \\
bigger on the section view \\
as well.
\end{tabular} & Images & 13 & 4 & 5 & 4.8 & 4 & 4 & 5 & 4.3 & 4.6 & 4.7 \\
\hline M Myer & MM644 & Internal & Content & \begin{tabular}{|l|} 
Controls \\
Strategies: \\
Vacancy \\
Sensors, \\
Daylight \\
Harvesting
\end{tabular} & & & & Plan Diagram & $\begin{array}{l}\text { Text in legend cannot be } \\
\text { read }\end{array}$ & Images & 13 & 4 & 5 & 4.8 & 4 & 4 & 5 & 4.3 & 4.6 & 4.7 \\
\hline N Ferzacca & NF11 & Engineer & & & & & & Controls details. & $\begin{array}{l}\text { The enlarged view is not } \\
\text { readable. }\end{array}$ & Images & 13 & 4 & 5 & 4.8 & 4 & 4 & 5 & 4.3 & 4.6 & 4.7 \\
\hline
\end{tabular}




\begin{tabular}{|c|c|c|c|c|c|c|c|c|c|c|c|c|c|c|c|c|c|c|c|c|}
\hline $\begin{array}{l}\text { Commenter } \\
\text { ID \# }\end{array}$ & $\begin{array}{l}\text { Comment } \\
\text { Reference } \\
\#\end{array}$ & $\begin{array}{l}\text { Commenter } \\
\text { Category }\end{array}$ & \begin{tabular}{|l} 
Comment \\
Type
\end{tabular} & Space Type & \begin{tabular}{|l|} 
Ceiling \\
Height/ \\
Total Area
\end{tabular} & \begin{tabular}{|l}
$\#$ \\
workstations/ \\
partition \\
height (open \\
office only) \\
\end{tabular} & \begin{tabular}{|l|} 
Daylight \\
Input (if \\
applicable)
\end{tabular} & \begin{tabular}{|l|} 
Reference webpage \\
or other location \\
(see \\
"Reference.doc")
\end{tabular} & $\begin{array}{l}\text { Comment Description: } \\
\text { Issues, Suggestions \& } \\
\text { Requests }\end{array}$ & Action/Solutior & \begin{tabular}{|l|} 
Status \\
or \\
Ticket \#
\end{tabular} & $\begin{array}{c}\text { Frequenc } \\
\mathrm{y}(20 \%)\end{array}$ & $\begin{array}{c}\text { Importance } \\
\text { of comment } \\
(80 \%)\end{array}$ & \begin{tabular}{|c|} 
Sum \\
$(100 \%)$
\end{tabular} & $\begin{array}{c}\text { Ease of } \\
\text { Implementatio } \\
\mathrm{n}(30 \%)\end{array}$ & $\begin{array}{c}\text { Duration } \\
(40 \%)\end{array}$ & $\begin{array}{c}\text { Risk/ } \\
\text { Complicatio } \\
\mathrm{n}(30 \%)\end{array}$ & \begin{tabular}{|c|} 
Sum \\
$(100 \%)$
\end{tabular} & $\begin{array}{c}\text { Short Term } \\
(40 \% \\
\text { Importance) } \\
(60 \% \text { Feasibility) } \\
\end{array}$ & $\begin{array}{c}\text { Long Term } \\
(85 \% \\
\text { Importance) } \\
\text { (15\% Feasibility) } \\
\end{array}$ \\
\hline M Myer & 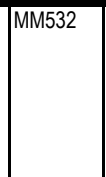 & Internal & Content & \begin{tabular}{|l|} 
Recessed Non- \\
Planar (LPD \\
0.64 W/sf)
\end{tabular} & $8 / 5000$ & $30 / 48$ & & $\begin{array}{l}\begin{array}{l}\text { Approach/Luminaire } \\
\text { s B }\end{array} \\
\end{array}$ & $\begin{array}{l}\text { The text is describing } \\
\text { Luminaire Type B as a task } \\
\text { light, yet the box to the right } \\
\text { is calling it an undercab. }\end{array}$ & Luminaires & 4 & 1 & 4 & 3.4 & 4 & 4 & 4 & 4 & 3.6 & 3.5 \\
\hline M Myer & MM519 & Internal & Content & \begin{tabular}{|l|} 
Recessed Non- \\
Planar (LPD \\
0.64 W/sf) \\
\end{tabular} & $8 / 5000$ & $30 / 48$ & & Luminaires C & $\begin{array}{l}\text { The LED undercabinet } \\
\text { fixture should produce } \\
\text { more lumens than the } \\
\text { articulated desktop } \\
\text { luminaire. }\end{array}$ & Luminaires & 4 & 1 & 4 & 3.4 & 4 & 4 & 4 & 4 & 3.6 & 3.5 \\
\hline J Yorgey & JY38 & Manufacturer & General & & & & & Overall & $\begin{array}{l}\text { Dimming ballasts not listed } \\
\text { on fixture schedule }\end{array}$ & Luminaires & 22 & 3 & 5 & 4.6 & 3 & 3 & 3 & 3 & 4.0 & 4.4 \\
\hline J Yorgey & JY26 & Manufacturer & & & & & & Fixture schedule & \begin{tabular}{|l|} 
Shows instart ballast type \\
even though dimming was \\
selected
\end{tabular} & Luminaires & 22 & 3 & 5 & 4.6 & 3 & 3 & 3 & 3 & 4.0 & 4.4 \\
\hline A Cortese & AC11 & Architect & Content & & & & & & \begin{tabular}{|l|} 
also noticed that some of \\
the language is incomplete, \\
(i.e. in the Suspended \\
Direct Indirect Lensed \\
Luminarie with high BF, the \\
number of lamps is \\
missing)
\end{tabular} & Luminaires & 4 & 2 & 4 & 3.6 & 5 & 4 & 5 & 4.6 & 4.0 & 3.8 \\
\hline HMcKay & HM42 & Designer & Content & & & & & & \begin{tabular}{|l|} 
All reception vignettes: \\
Type A not described in \\
side bar of any vignettes. \\
Task light described in \\
strategy but not shown on \\
plan or \\
sidebar.
\end{tabular} & Luminaires & 4 & 2 & 4 & 3.6 & 5 & 4 & 5 & 4.6 & 4.0 & 3.8 \\
\hline HMcKay & HM28 & Designer & Content & & & & & & $\begin{array}{l}\text { V2 - Side bar calls for Type } \\
\text { B undercabinet lights even } \\
\text { though partitions are low. }\end{array}$ & Luminaires & 4 & 2 & 4 & 3.6 & 5 & 4 & 5 & 4.6 & 4.0 & 3.8 \\
\hline HMcKay & HM5 & Designer & \begin{tabular}{|l} 
Content \\
\end{tabular} & & & & & & \begin{tabular}{|l|} 
Luminaire side-bars. Need \\
"fixture schedule type"
\end{tabular} & Luminaires & 4 & 2 & 4 & 3.6 & 5 & 4 & 5 & 4.6 & 4.0 & 3.8 \\
\hline M Myer & MM593 & Internal & Content & \begin{tabular}{|l|} 
Perimter \\
Recessed CFLs
\end{tabular} & & & & Luminaires & $\begin{array}{l}\text { For the MLPW calculation, } \\
\text { we started off with a lamp } \\
\text { that was } 1800 \text { lumens? }\end{array}$ & Luminaires & 4 & 2 & 4 & 3.6 & 5 & 4 & 5 & 4.6 & 4.0 & 3.8 \\
\hline M Myer & MM518 & Internal & Content & \begin{tabular}{|l|} 
Recessed Non- \\
Planar (LPD \\
0.64 W/sf) \\
\end{tabular} & $8 / 5000$ & $30 / 48$ & & Luminaires C & $\begin{array}{l}\text { How is MLPW calculated } \\
\text { for the LEDs? What value } \\
\text { are you using for mean } \\
\text { lumens? By my math } 36.0 \\
\text { MLPW }{ }^{*} .2 \mathrm{~W}=324 \text { mean } \\
\text { lumens. I looks like this } \\
\text { product is only producing } \\
324 \text { lumens. I think that we } \\
\text { need some dimensions for } \\
\text { the luminaire }\end{array}$ & Luminaires & 4 & 2 & 4 & 3.6 & 5 & 4 & 5 & 4.6 & 4.0 & 3.8 \\
\hline
\end{tabular}




\begin{tabular}{|c|c|c|c|c|c|c|c|c|c|c|c|c|c|c|c|c|c|c|c|c|}
\hline $\begin{array}{l}\text { Commenter } \\
\text { ID \# }\end{array}$ & $\begin{array}{l}\text { Comment } \\
\text { Reference } \\
\#\end{array}$ & $\begin{array}{l}\text { Commenter } \\
\text { Category }\end{array}$ & \begin{tabular}{|l} 
Comment \\
Type
\end{tabular} & Space Type & \begin{tabular}{|l|} 
Ceiling \\
Height// \\
Total Area
\end{tabular} & \begin{tabular}{|l|} 
\\
workstations/ \\
partition \\
height (open \\
office only) \\
\end{tabular} & $\mid \begin{array}{l}\text { Daylight } \\
\text { Input (if } \\
\text { applicable) }\end{array}$ & $\begin{array}{l}\text { Reference webpage } \\
\text { or other location } \\
\text { (see } \\
\text { "Reference.doc") }\end{array}$ & \begin{tabular}{|l|} 
Comment Description: \\
Issues, Suggestions \& \\
Requests
\end{tabular} & Action/Solution & \begin{tabular}{|l|}
$\begin{array}{l}\mid l \\
\text { tatus } \\
\text { or } \\
\text { Ticket \# }\end{array}$ \\
\end{tabular} & $\begin{array}{c}\text { Frequenc } \\
\mathrm{y}(20 \%)\end{array}$ & $\begin{array}{c}\text { Importance } \\
\text { of comment } \\
(80 \%)\end{array}$ & \begin{tabular}{|c|} 
Sum \\
$(100 \%)$
\end{tabular} & $\begin{array}{c}\text { Ease of } \\
\text { Implementatio } \\
\mathrm{n}(30 \%)\end{array}$ & $\begin{array}{c}\text { Duration } \\
(40 \%)\end{array}$ & $\begin{array}{c}\text { Risk/ } \\
\text { Complicatio } \\
\mathrm{n}(30 \%)\end{array}$ & $\begin{array}{c}\text { Sum } \\
(100 \%)\end{array}$ & $\begin{array}{c}\begin{array}{c}\text { Short Term } \\
(40 \% \\
\text { Importance) } \\
(60 \% \text { Feasibility) }\end{array} \\
\end{array}$ & $\begin{array}{c}\text { Long Term } \\
(85 \% \\
\text { Importance) } \\
\text { (15\% Feasibility) } \\
\end{array}$ \\
\hline M Myer & MM463 & Internal & \begin{tabular}{|l|} 
Appearancel \\
Aesthetics
\end{tabular} & $\begin{array}{l}\text { Recessed Non- } \\
\text { Planar on wider } \\
\text { centers }\end{array}$ & $9.6 / 5000$ & $40 / 48$ & & Luminaires $\mathrm{A}$ & $\begin{array}{l}\text { The calculation for MLPW } \\
\text { looks high. Here is my } \\
\text { math: } 2^{*} 3100^{*} 0.88^{*} 0.95 / 55 \\
=94 \text { MLPW }\end{array}$ & Luminaires & 4 & 2 & 4 & 3.6 & 5 & 4 & 5 & 4.6 & 4.0 & 3.8 \\
\hline M Myer & MM337 & Internal & Content & \begin{tabular}{|l|l} 
Suspended \\
Direct/Indirect \\
Luminaires with \\
low BF ballasts
\end{tabular} & $9^{\prime}$ & PO & & Luminaires & $\begin{array}{l}\text { The MLPW value is wrong. } \\
\text { I think that it should be } \\
3100^{*} 2^{*} .95(L L D)^{*} 0.78 / 48 \\
=102 \text { MLPW. I do not know } \\
\text { how you calculated the } 122 \\
\text { MLPW }\end{array}$ & \begin{tabular}{|l} 
Luminaires \\
\end{tabular} & 4 & 2 & 4 & 3.6 & 5 & 4 & 5 & 4.6 & 4.0 & 3.8 \\
\hline N Miller & NM18 & Internal & & & & & & & \begin{tabular}{|l|} 
Check significant digits on \\
MLPW for luminaires.
\end{tabular} & Luminaires & 4 & 2 & 4 & 3.6 & 5 & 4 & 5 & 4.6 & 4.0 & 3.8 \\
\hline N Miller & NM9 & Internal & General & \begin{tabular}{|l|}
$\begin{array}{l}\text { Pharmacy Des } \\
\text { Vignettes }\end{array}$ \\
\end{tabular} & & & & \begin{tabular}{|l|} 
Vignettes for \\
Perimeter - details \\
of skylt + perimeter \\
Itg
\end{tabular} & \begin{tabular}{|l|} 
Is the lighting plan \\
supposed to be cut off on \\
the edges? It looks kinda \\
funny. \\
Would it be better to \\
illustrate the skylights \\
illuminating a \\
merchandising wall instead \\
of a window wall? It would \\
make the skylights look \\
more important. \\
Luminaires - Should the \\
1 x4 wallwashers have an \\
asymmetrical reflector \\
rather than symmetrical? If \\
so, wouldn't the efficiency \\
be less that 90\%? Maybe \\
I'm misinterpreting the \\
"angled" reffector luminaire \\
description.
\end{tabular} & \begin{tabular}{|l} 
Luminaires \\
\\
\end{tabular} & 4 & 2 & 4 & 3.6 & 5 & 4 & 5 & 4.6 & 4.0 & 3.8 \\
\hline \begin{tabular}{|l}
$J$ Beutell \\
\end{tabular} & JB3 & Owner & & & & & & & limit your decimal points. & Luminaires & 23 & 2 & 5 & 4.4 & 4 & 4 & 4 & 4 & 4.2 & 4.3 \\
\hline M Myer & MM72 & Internal & Content & \begin{tabular}{|l|} 
Recessed Slot \\
Lumianirs \\
parallel to desk \\
with compact \\
fluorescent wall- \\
washers (office)
\end{tabular} & & & & Luminaire $B$ & \begin{tabular}{|l|}
28 watts for a $26 \mathrm{~W}$ CFL \\
seems low, I would go with \\
29. I also would remove the \\
decimal for all power \\
values
\end{tabular} & \begin{tabular}{|l} 
Luminaires \\
\end{tabular} & 23 & 2 & 5 & 4.4 & 4 & 4 & 4 & 4 & 4.2 & 4.3 \\
\hline M Myer & MM142 & Internal & Content & \begin{tabular}{|l|} 
Recessed \\
Compact \\
Fluorescent \\
Downlights with \\
Dropped \\
Decorative \\
Diffuser and \\
Perimeter Wall- \\
Slot (office) \\
\end{tabular} & & & & Luminaires/B & $\begin{array}{l}\text { Add a significant digit for } \\
\text { luminaire efficiency }\end{array}$ & Luminaires & 23 & 2 & 5 & 4.4 & 4 & 4 & 4 & 4 & 4.2 & 4.3 \\
\hline
\end{tabular}




\begin{tabular}{|c|c|c|c|c|c|c|c|c|c|c|c|c|c|c|c|c|c|c|c|c|}
\hline $\begin{array}{l}\text { Commenter } \\
\text { ID\# }\end{array}$ & \begin{tabular}{|l|} 
Comment \\
Reference \\
$\#$
\end{tabular} & $\begin{array}{l}\text { Commenter } \\
\text { Category }\end{array}$ & \begin{tabular}{|l} 
Comment \\
Type
\end{tabular} & Space Type & $\mid \begin{array}{l}\text { Ceiling } \\
\text { Height/ } \\
\text { Total Area }\end{array}$ & \begin{tabular}{|l}
$\#$ \\
workstations/ \\
partition \\
height (open \\
office only) \\
\end{tabular} & $\mid \begin{array}{l}\text { Daylight } \\
\text { Input (if } \\
\text { applicable) }\end{array}$ & \begin{tabular}{|l|} 
Reference webpage \\
or other location \\
(see \\
"Reference.doc") \\
\end{tabular} & $\begin{array}{l}\text { Comment Description: } \\
\text { Issues, Suggestions \& } \\
\text { Requests }\end{array}$ & Action/Solution & \begin{tabular}{|l|} 
Status \\
or \\
Ticket \#
\end{tabular} & $\begin{array}{c}\text { Frequenc } \\
\text { y (20\%) }\end{array}$ & $\begin{array}{c}\text { Importance } \\
\text { of comment } \\
(80 \%)\end{array}$ & $\begin{array}{c}\text { Sum } \\
(100 \%)\end{array}$ & $\begin{array}{c}\text { Ease of } \\
\text { Implementatio } \\
\mathrm{n}(30 \%)\end{array}$ & $\begin{array}{c}\text { Duration } \\
(40 \%)\end{array}$ & $\mid \begin{array}{c}\text { Risk/ } \\
\text { Complicatio } \\
\mathrm{n}(30 \%)\end{array}$ & \begin{tabular}{|} 
Sum \\
$(100 \%)$
\end{tabular} \mid & \begin{tabular}{|c|} 
Short Term \\
$(40 \%$ \\
Importance) \\
$(60 \%$ Feasibility) \\
\end{tabular} & $\begin{array}{c}\text { Long Term } \\
(85 \% \\
\text { Importance) } \\
\text { (15\% Feasibility) }\end{array}$ \\
\hline M Myer & MM172 & | Internal & Content & \begin{tabular}{|l|} 
Recessed slot \\
luminaires \\
Perpendicular \\
to Desk with \\
Compact \\
Fluorescent \\
Downlights and \\
Wall-washers \\
(Office)
\end{tabular} & & & & Luminaires/B & \begin{tabular}{|l} 
Express luminaire \\
efficiency as two digits
\end{tabular} & Luminaires & 23 & 2 & 5 & 4.4 & 4 & 4 & 4 & 4 & 4.2 & 4.3 \\
\hline M Myer & MM62 & Internal & Content & \begin{tabular}{|l|} 
Recessed Slot \\
Lumianirs \\
parallel to desk \\
with compact \\
fluorescent wall- \\
washers (office)
\end{tabular} & & & & Luminaire $\mathrm{A}$ & $\begin{array}{l}\text { I have never seen CRI } \\
\text { presented as a significant } \\
\text { digit. I would remove the } \\
\text { decimal. }\end{array}$ & Luminaires & 23 & 2 & 5 & 4.4 & 4 & 4 & 4 & 4 & 4.2 & 4.3 \\
\hline M Myer & MM187 & Internal & Content & \begin{tabular}{|l|} 
Recessed slot \\
luminaires \\
Perpendicular \\
to Desk with \\
Compact \\
Fluorescent \\
Downlights and \\
Wall-washers \\
(Office)
\end{tabular} & & & & Luminaires/D & $\begin{array}{l}\text { Input power should be } \\
\text { written as a whole number }\end{array}$ & Luminaires & 23 & 2 & 5 & 4.4 & 4 & 4 & 4 & 4 & 4.2 & 4.3 \\
\hline M Myer & MM183 & Internal & Content & \begin{tabular}{|l|} 
Recessed slot \\
luminaires \\
Perpendicular \\
to Desk with \\
Compact \\
Fluorescent \\
Downlights and \\
Wall-washers \\
(Office)
\end{tabular} & & & & Luminaires/D & $\begin{array}{l}\text { MLPW should be } \\
\text { expressed as a whole } \\
\text { number }\end{array}$ & Luminaires & 23 & 2 & 5 & 4.4 & 4 & 4 & 4 & 4 & 4.2 & 4.3 \\
\hline M Myer & MM612 & Internal & Content & \begin{tabular}{|l} 
Perimeter \\
Recessed \\
Linear \\
Fluorescent
\end{tabular} & & & & Luminaires & $\begin{array}{l}\text { Please make } \mathrm{CRI} \text { a whole } \\
\text { number }\end{array}$ & Luminaires & 23 & 2 & 5 & 4.4 & 4 & 4 & 4 & 4 & 4.2 & 4.3 \\
\hline M Myer & MM613 & Internal & Content & \begin{tabular}{|l} 
Perimeter \\
Recessed \\
Linear \\
Fluorescent
\end{tabular} & & & & Luminaires & $\begin{array}{l}\text { Please make input power a } \\
\text { whole number }\end{array}$ & Luminaires & 23 & 2 & 5 & 4.4 & 4 & 4 & 4 & 4 & 4.2 & 4.3 \\
\hline M Myer & MM614 & Internal & Content & \begin{tabular}{|l} 
Perimeter \\
Recessed \\
Linear \\
Fluorescent
\end{tabular} & & & & Luminaires & $\begin{array}{l}\text { Please make MLPW a } \\
\text { whole number }\end{array}$ & Luminaires & 23 & 2 & 5 & 4.4 & 4 & 4 & 4 & 4 & 4.2 & 4.3 \\
\hline M Myer & MM431 & Internal & Content & $\begin{array}{l}\begin{array}{l}\text { Suspended 12' } \\
\text { single-lamp }\end{array} \\
\end{array}$ & $9.6 / 5000$ & $40 / 48$ & & Luminaires B & $\begin{array}{l}\text { Please make the input } \\
\text { power a whole number }\end{array}$ & Luminaires & 23 & 2 & 5 & 4.4 & 4 & 4 & 4 & 4 & 4.2 & 4.3 \\
\hline
\end{tabular}




\begin{tabular}{|c|c|c|c|c|c|c|c|c|c|c|c|c|c|c|c|c|c|c|c|c|}
\hline $\begin{array}{l}\text { Commenter } \\
\text { ID \# }\end{array}$ & \begin{tabular}{|l|} 
Comment \\
Reference \\
$\#$
\end{tabular} & $\begin{array}{l}\text { Commenter } \\
\text { Category }\end{array}$ & \begin{tabular}{|l} 
Comment \\
Type
\end{tabular} & Space Type & \begin{tabular}{|l|} 
Ceiling \\
Height/ \\
Total Area
\end{tabular} & \begin{tabular}{|l}
$\#$ \\
workstations/ \\
partition \\
height (open \\
office only) \\
\end{tabular} & \begin{tabular}{|l} 
Daylight \\
Input (if \\
applicable)
\end{tabular} & \begin{tabular}{|l|} 
Reference webpage \\
or other location \\
(see \\
"Reference.doc")
\end{tabular} & $\begin{array}{l}\text { Comment Description: } \\
\text { Issues, Suggestions \& } \\
\text { Requests }\end{array}$ & Action/Solution & \begin{tabular}{|l|} 
Status \\
or \\
Ticket \#
\end{tabular} & $\begin{array}{c}\text { Frequenc } \\
\mathrm{y}(20 \%)\end{array}$ & $\begin{array}{c}\text { Importance } \\
\text { of comment } \\
(80 \%)\end{array}$ & \begin{tabular}{|l|} 
Sum \\
$(100 \%)$
\end{tabular} & $\begin{array}{c}\text { Ease of } \\
\text { Implementatio } \\
\mathrm{n}(30 \%)\end{array}$ & $\begin{array}{c}\text { Duration } \\
(40 \%)\end{array}$ & $\begin{array}{c}\text { Risk/ } \\
\text { Complicatio } \\
\mathrm{n}(30 \%)\end{array}$ & \begin{tabular}{|c|} 
Sum \\
$(100 \%)$
\end{tabular} & $\begin{array}{c}\text { Short Term } \\
(40 \% \\
\text { Importance) } \\
(60 \% \text { Feasibility })\end{array}$ & $\begin{array}{c}\text { Long Term } \\
(85 \% \\
\text { Importance) } \\
\text { (15\% Feasibility) }\end{array}$ \\
\hline$\longdiv { \text { N Ferzacca } }$ & NF7 & Engineer & & & & & & & Round your decimal points. & Luminaires & 23 & 2 & 5 & 4.4 & 4 & 4 & 4 & 4 & 4.2 & 4.3 \\
\hline N Miller & NM11 & Internal & & & & & & & \begin{tabular}{|l|} 
Check \# of significant \\
digits in MLPW under \\
Luminaires
\end{tabular} & Luminaires & 23 & 2 & 5 & 4.4 & 4 & 4 & 4 & 4 & 4.2 & 4.3 \\
\hline M Myer & MM277 & Internal & Content & $\begin{array}{l}\text { Recessed 4' } \\
\text { Asymmetric } \\
\text { wall-wash } \\
\text { luminaires }\end{array}$ & & & \begin{tabular}{|l|} 
corridors \\
\end{tabular} & Luminaires & \begin{tabular}{|l} 
What an instant-start \\
ballast. I think that you \\
would want a PRS ballast
\end{tabular} & Luminaires & 4 & 1 & 5 & 4.2 & 4 & 5 & 4 & 4.4 & 4.3 & 4.2 \\
\hline M Myer & MM139 & Internal & Content & \begin{tabular}{|l|} 
Recessed \\
Compact \\
Fluorescent \\
Downlights with \\
Dropped \\
Decorative \\
Diffuser and \\
Perimeter Wall- \\
Slot (office) \\
\end{tabular} & & & & Luminaires/A & $\begin{array}{l}\text { I believe that PRS is the } \\
\text { standard for CFLs not IS }\end{array}$ & Luminaires & 4 & 1 & 5 & 4.2 & 4 & 5 & 4 & 4.4 & 4.3 & 4.2 \\
\hline M Myer & MM588 & Internal & Content & \begin{tabular}{|l|} 
Perimter \\
Recessed CFLS
\end{tabular} & & & & Luminaires & $\begin{array}{l}\text { I believe the default CFL } \\
\text { ballast is PRS not Instant } \\
\text { start }\end{array}$ & Luminaires & 4 & 1 & 5 & 4.2 & 4 & 5 & 4 & 4.4 & 4.3 & 4.2 \\
\hline M Myer & \begin{tabular}{|l|} 
MM587 \\
\end{tabular} & Internal & Content & \begin{tabular}{|l|} 
Perimter \\
Recessed CFLS
\end{tabular} & & & & Luminaires & $\begin{array}{l}\text { I do not believe NEMA } \\
\text { premium ballasts exist for } \\
\text { CFLs }\end{array}$ & Luminaires & 4 & 1 & 5 & 4.2 & 4 & 5 & 4 & 4.4 & 4.3 & 4.2 \\
\hline M Myer & \begin{tabular}{|l|} 
MM71 \\
\end{tabular} & Internal & Content & \begin{tabular}{|l|} 
Recessed Slot \\
Lumianirs \\
parallel to desk \\
with compact \\
fluorescent wall- \\
washers (office)
\end{tabular} & & & & Luminaire B & $\begin{array}{l}\text { Isn't PRS the default CFL } \\
\text { ballast type and instant- } \\
\text { start is the outlier? }\end{array}$ & Luminaires & 4 & 1 & 5 & 4.2 & 4 & 5 & 4 & 4.4 & 4.3 & 4.2 \\
\hline M Myer & MM106 & Internal & Content & \begin{tabular}{|l|} 
Recessed Slot \\
Luminaires \\
Parallel to Desk \\
with \\
Contiunous \\
Linear Wall- \\
washers (office)
\end{tabular} & & & & Luminaires C & $\begin{array}{l}\text { Why is the luminaire } \\
\text { efficiency so low for this } \\
\text { luminaire type? Also, let's } \\
\text { use } 2 \text { significant digits here } \\
\text { like we have everywhere } \\
\text { else. }\end{array}$ & Luminaires & 4 & 1 & 5 & 4.2 & 4 & 5 & 4 & 4.4 & 4.3 & 4.2 \\
\hline M Myer & MM330 & Internal & Content & \begin{tabular}{|l|} 
Suspended \\
Direct/Indirect \\
Luminaires with \\
low BF ballasts \\
\end{tabular} & $9^{\prime}$ & PO & & Luminaires & $\begin{array}{l}\text { "Two-lamp profile" - needs } \\
\text { the word "in" and probably } \\
\text { "T8" }\end{array}$ & Luminaires & 4 & 1 & 5 & 4.2 & 4 & 5 & 4 & 4.4 & 4.3 & 4.2 \\
\hline
\end{tabular}




\begin{tabular}{|c|c|c|c|c|c|c|c|c|c|c|c|c|c|c|c|c|c|c|c|c|}
\hline $\begin{array}{l}\text { Commenter } \\
\text { ID\# }\end{array}$ & \begin{tabular}{|l|} 
Comment \\
Reference \\
$\#$
\end{tabular} & $\begin{array}{l}\text { Commenter } \\
\text { Category }\end{array}$ & \begin{tabular}{|l} 
Comment \\
Type
\end{tabular} & Space Type & $\mid \begin{array}{l}\text { Ceiling } \\
\text { Height/ } \\
\text { Total Area }\end{array}$ & \begin{tabular}{|l}
$\#$ \\
workstations/ \\
partition \\
height (open \\
office only) \\
\end{tabular} & $\mid \begin{array}{l}\text { Daylight } \\
\text { Input (if } \\
\text { applicable) }\end{array}$ & \begin{tabular}{|l|} 
Reference webpage \\
or other location \\
(see \\
"Reference.doc")
\end{tabular} & $\begin{array}{l}\text { Comment Description: } \\
\text { Issues, Suggestions \& } \\
\text { Requests }\end{array}$ & Action/Solution & \begin{tabular}{|l|} 
Status \\
or \\
Ticket \#
\end{tabular} & $\mid \begin{array}{c}\text { Frequenc } \\
\mathrm{y}(20 \%)\end{array}$ & $\begin{array}{c}\text { Importance } \\
\text { of comment } \\
(80 \%)\end{array}$ & \begin{tabular}{|c|} 
Sum \\
$(100 \%)$
\end{tabular} & $\begin{array}{c}\text { Ease of } \\
\text { Implementatio } \\
\mathrm{n}(30 \%)\end{array}$ & \begin{tabular}{|c|} 
Duration \\
$(40 \%)$
\end{tabular} & $\begin{array}{c}\text { Risk/ } \\
\text { Complicatio } \\
\mathrm{n}(30 \%)\end{array}$ & \begin{tabular}{|} 
Sum \\
$(100 \%)$
\end{tabular} \mid & \begin{tabular}{|c|} 
Short Term \\
$(40 \%$ \\
Importance) \\
$(60 \%$ Feasibility) \\
\end{tabular} & $\begin{array}{c}\text { Long Term } \\
(85 \% \\
\text { Importance) } \\
\text { (15\% Feasibility) }\end{array}$ \\
\hline M Myer & MM170 & | Internal & Content & \begin{tabular}{|l|} 
Recessed slot \\
luminaires \\
Perpendicular \\
to Desk with \\
Compact \\
Fluorescent \\
Downlights and \\
Wall-washers \\
(Office)
\end{tabular} & & & & Luminaires/B & $\begin{array}{l}\text { CFLs typically use PRS } \\
\text { ballasts and not instant } \\
\text { start ballasts }\end{array}$ & Luminaires & 4 & 1 & 5 & 4.2 & 4 & 5 & 4 & 4.4 & 4.3 & 4.2 \\
\hline M Myer & MM459 & Internal & \begin{tabular}{|l|} 
Appearancel \\
Aesthetics
\end{tabular} & $\begin{array}{l}\text { Recessed Non- } \\
\text { Planar on wider } \\
\text { centers }\end{array}$ & $9.6 / 5000$ & $40 / 48$ & & Luminaires $\mathrm{A}$ & $\begin{array}{l}\text { lassume that this is a 2- } \\
\text { lamp fixture, but it is not } \\
\text { obvious }\end{array}$ & Luminaires & 4 & 1 & 5 & 4.2 & 4 & 5 & 4 & 4.4 & 4.3 & 4.2 \\
\hline M Myer & MM67 & Internal & Content & \begin{tabular}{|l|} 
Recessed Slot \\
Lumianirs \\
parallel to desk \\
with compact \\
fluorescent wall- \\
washers (office)
\end{tabular} & & & & Luminaire B & $\begin{array}{l}\text { I would put recessed first, } \\
\text { e.g. "Recessed round 6" } \\
\text { aperture lensed-CFL wall } \\
\text { washer" }\end{array}$ & \begin{tabular}{|l|l|} 
Luminaires \\
\end{tabular} & 4 & 1 & 5 & 4.2 & 4 & 5 & 4 & 4.4 & 4.3 & 4.2 \\
\hline M Myer & \begin{tabular}{|l|} 
MM426 \\
\end{tabular} & Internal & Content & \begin{tabular}{|l}
$\begin{array}{l}\text { Suspended 12' } \\
\text { single-lamp }\end{array}$ \\
\end{tabular} & $9.6 / 5000$ & $40 / 48$ & & Luminaires B & $\begin{array}{l}\text { I would put T8 after One- } \\
\text { lamp and I would add "In" } \\
\text { before profile }\end{array}$ & \begin{tabular}{|l|l|} 
Luminaires \\
\end{tabular} & 4 & 1 & 5 & 4.2 & 4 & 5 & 4 & 4.4 & 4.3 & 4.2 \\
\hline M Myer & MM160 & Internal & Content & \begin{tabular}{|l|} 
Recessed slot \\
luminaires \\
Perpendicular \\
to Desk with \\
Compact \\
Fluorescent \\
Downlights and \\
Wall-washers \\
(Office)
\end{tabular} & & & & Luminaires/A & Move T8 after one-lamp & \begin{tabular}{|l|l|} 
Luminaires \\
\end{tabular} & 17 & 1 & 5 & 4.2 & 4 & 5 & 4 & 4.4 & 4.3 & 4.2 \\
\hline M Myer & MM60 & Internal & Content & \begin{tabular}{|l|} 
Recessed Slot \\
Lumianirs \\
parallel to desk \\
with compact \\
fluorescent wall- \\
washers (office)
\end{tabular} & & & & Luminaire A & $\begin{array}{l}\text { Recommend moving the T8 } \\
\text { after the "one lamp". Why is } \\
\text { "One" capitalized? }\end{array}$ & \begin{tabular}{|l|l|} 
Luminaires \\
\end{tabular} & 17 & 1 & 5 & 4.2 & 4 & 5 & 4 & 4.4 & 4.3 & 4.2 \\
\hline M Myer & MM513 & Internal & Content & \begin{tabular}{|l|} 
Recessed Non- \\
Planar (LPD \\
0.64 W/sf) \\
\end{tabular} & $8 / 5000$ & $30 / 48$ & & Luminaires C & $\begin{array}{l}\text { Shouldn't warm-white be } \\
\text { hyphenated? }\end{array}$ & Luminaires & 17 & 1 & 5 & 4.2 & 4 & 5 & 4 & 4.4 & 4.3 & 4.2 \\
\hline
\end{tabular}


Appendix E: Master Comments List

\begin{tabular}{|c|c|c|c|c|c|c|c|c|c|c|c|c|c|c|c|c|c|c|c|c|}
\hline $\begin{array}{l}\text { Commenter } \\
\text { ID \# }\end{array}$ & \begin{tabular}{|l|} 
Comment \\
Reference \\
$\#$
\end{tabular} & $\begin{array}{l}\text { Commenter } \\
\text { Category }\end{array}$ & \begin{tabular}{|l} 
Comment \\
Type
\end{tabular} & Space Type & \begin{tabular}{|l} 
Ceiling \\
Height// \\
Total Area
\end{tabular} & \begin{tabular}{|l|}
$\#$ \\
workstations/ \\
partition \\
height (open \\
office only) \\
\end{tabular} & \begin{tabular}{|l|} 
Daylight \\
Input (if \\
applicable)
\end{tabular} & \begin{tabular}{|l|} 
Reference webpage \\
or other location \\
(see \\
"Reference.doc") \\
\end{tabular} & \begin{tabular}{|l|} 
Comment Description: \\
Issues, Suggestions \& \\
Requests
\end{tabular} & Action/Solution & \begin{tabular}{|l|} 
Status \\
or \\
Ticket \#
\end{tabular} & $\begin{array}{c}\text { Frequenc } \\
\mathrm{y}(20 \%)\end{array}$ & $\begin{array}{c}\text { Importance } \\
\text { of comment } \\
(80 \%)\end{array}$ & $\begin{array}{c}\text { Sum } \\
(100 \%)\end{array}$ & $\begin{array}{c}\text { Ease of } \\
\text { Implementatio } \\
\mathrm{n}(30 \%)\end{array}$ & $\begin{array}{c}\text { Duration } \\
(40 \%)\end{array}$ & $\begin{array}{c}\text { Risk/ } \\
\text { Complicatio } \\
\mathrm{n}(30 \%)\end{array}$ & \begin{tabular}{|} 
Sum \\
$(100 \%)$
\end{tabular} & \begin{tabular}{|c|} 
Short Term \\
$(40 \%$ \\
Importance) \\
(60\% Feasibility)
\end{tabular} & $\begin{array}{c}\text { Long Term } \\
(85 \% \\
\text { Importance) } \\
\text { (15\% Feasibility) } \\
\end{array}$ \\
\hline M Myer & MM608 & Internal & Content & $\begin{array}{l}\text { Perimeter } \\
\text { Recessed } \\
\text { Linear } \\
\text { Fluorescent }\end{array}$ & & & & Luminaires & \begin{tabular}{|l|} 
The title of the vignette \\
hyphenates wall washing \\
which I do not believe is \\
correct. The words really \\
only need to be \\
hyphenated when it is a \\
compound adjective (e.g., if \\
one word in the adjective \\
cannot stand alone). Here \\
we says "wall wash \\
luminaire" - this would be a \\
case where hyphenating \\
would make more sense
\end{tabular} & Luminaires & 17 & 1 & 5 & 4.2 & 4 & 5 & 4 & 4.4 & 4.3 & 4.2 \\
\hline M Myer & MM418 & Internal & Content & \begin{tabular}{|l|}
$\begin{array}{l}\text { Suspended 12' } \\
\text { single-lamp }\end{array}$ \\
\end{tabular} & $9.6 / 5000$ & $40 / 48$ & & Luminaires $\mathrm{A}$ & \begin{tabular}{|l} 
Luminaire A is "pendant- \\
mounted" and luminaire B \\
is "Suspended" - can they \\
be both the same wording?
\end{tabular} & Luminaires & 3 & 2 & 5 & 4.4 & 4 & 5 & 4 & 4.4 & 4.4 & 4.4 \\
\hline M Myer & MM86 & Internal & Content & \begin{tabular}{|l|} 
Recessed Slot \\
Lumianirs \\
parallel to desk \\
with compact \\
fluorescent wall- \\
washers (office)
\end{tabular} & & & & Strategy/luminaires & \begin{tabular}{|l|} 
Luminaire C - I think that \\
the i.e. of articulated is a bit \\
much, the clarification \\
should stop after \\
"supplment the ambient \\
lighting". I would then \\
modify the text about the \\
blind old person wanting \\
more light
\end{tabular} & Luminaires & 3 & 2 & 5 & 4.4 & 4 & 5 & 4 & 4.4 & 4.4 & 4.4 \\
\hline HMcKay & HM10 & Designer & Content & & & & & & $\begin{array}{l}\text { Luminaire Sidebars: Some } \\
\text { missing data altogether. All } \\
\text { are missing the \# of lamps. } \\
\text { Efficiency represented as } \\
\text { decimal rather than } \\
\text { percentage. MLPW is a } \\
\text { spreadsheet formula, not a } \\
\text { value. }\end{array}$ & Luminaires & 4 & 2 & 5 & 4.4 & 4 & 5 & 4 & 4.4 & 4.4 & 4.4 \\
\hline HMcKay & HM46 & Designer & Content & & & & & & $\begin{array}{l}\text { V4 - Sidebar data missing } \\
\text { for } A \text { and } B\end{array}$ & Luminaires & 4 & 2 & 5 & 4.4 & 4 & 5 & 4 & 4.4 & 4.4 & 4.4 \\
\hline M Myer & MM625 & Internal & Content & \begin{tabular}{|l} 
Perimeter \\
Recessed \\
Linear \\
Fluorescent
\end{tabular} & & & & Strategy/Luminaires & $\begin{array}{l}\text { "Perforated shield" - what is } \\
\text { the shield? What fixture is } \\
\text { this describing? }\end{array}$ & Luminaires & 4 & 2 & 5 & 4.4 & 4 & 5 & 4 & 4.4 & 4.4 & 4.4 \\
\hline M Myer & MM174 & Internal & Content & \begin{tabular}{|l|} 
Recessed slot \\
luminaires \\
Perpendicular \\
to Desk with \\
Compact \\
Fluorescent \\
Downlights and \\
Wall-washers \\
(Office)
\end{tabular} & & & & Luminaires/C & \begin{tabular}{|l|} 
\# of lamps in cross section \\
is missing.
\end{tabular} & Luminaires & 4 & 2 & 5 & 4.4 & 4 & 5 & 4 & 4.4 & 4.4 & 4.4 \\
\hline
\end{tabular}




\begin{tabular}{|c|c|c|c|c|c|c|c|c|c|c|c|c|c|c|c|c|c|c|c|c|}
\hline $\begin{array}{l}\text { Commenter } \\
\text { ID \# }\end{array}$ & \begin{tabular}{|l|} 
Comment \\
Reference \\
$\#$
\end{tabular} & $\begin{array}{l}\text { Commenter } \\
\text { Category }\end{array}$ & \begin{tabular}{|l} 
Comment \\
Type
\end{tabular} & Space Type & \begin{tabular}{|l|} 
Ceiling \\
Height/ \\
Total Area
\end{tabular} & \begin{tabular}{|l}
$\#$ \\
workstations/ \\
partition \\
height (open \\
office only) \\
\end{tabular} & $\mid \begin{array}{l}\text { Daylight } \\
\text { Input (if } \\
\text { applicable) }\end{array}$ & $\begin{array}{l}\text { Reference webpage } \\
\text { or other location } \\
\text { (see } \\
\text { "Reference.doc") }\end{array}$ & $\begin{array}{l}\text { Comment Description: } \\
\text { Issues, Suggestions \& } \\
\text { Requests }\end{array}$ & Action/Solution & \begin{tabular}{|l|}
$\begin{array}{l}\text { Status } \\
\text { or } \\
\text { Ticket \# }\end{array}$ \\
\end{tabular} & $\begin{array}{c}\text { Frequenc } \\
\text { y (20\%) }\end{array}$ & $\begin{array}{c}\text { Importance } \\
\text { of comment } \\
(80 \%)\end{array}$ & \begin{tabular}{|c|} 
Sum \\
$(100 \%)$
\end{tabular} & $\begin{array}{c}\text { Ease of } \\
\text { Implementatio } \\
\mathrm{n}(30 \%)\end{array}$ & $\begin{array}{c}\text { Duration } \\
(40 \%)\end{array}$ & $\begin{array}{c}\text { Risk/ } \\
\text { Complicatio } \\
\mathrm{n}(30 \%)\end{array}$ & \begin{tabular}{|} 
Sum \\
$(100 \%)$
\end{tabular} \mid & \begin{tabular}{|c|} 
Short Term \\
$(40 \%$ \\
Importance) \\
$(60 \%$ Feasibility) \\
\end{tabular} & $\begin{array}{c}\text { Long Term } \\
(85 \% \\
\text { Importance) } \\
\text { (15\% Feasibility) }\end{array}$ \\
\hline M Myer & $\overline{~ M M 494}$ & Internal & Content & \begin{tabular}{|l|} 
Recessed Non- \\
Planar (LPD \\
0.64 W/sf) \\
\end{tabular} & $8 / 5000$ & $30 / 48$ & & Luminaires $\mathrm{A}$ & \begin{tabular}{|l|} 
I would revise the text to \\
say "Recessed 2'x4"' Non- \\
Planar lensed luminaire.
\end{tabular} & Luminaires & 4 & 2 & 5 & 4.4 & 4 & 5 & 4 & 4.4 & 4.4 & 4.4 \\
\hline M Myer & MM272 & Internal & Content & $\begin{array}{l}\text { Recessed 4' } \\
\text { Asymmetric } \\
\text { wall-wash } \\
\text { luminaires }\end{array}$ & & & corridors & Luminaires & $\begin{array}{l}\text { If wall-wash luminaire is } \\
\text { hyphenated in the title, we } \\
\text { should do it in this box as } \\
\text { well. }\end{array}$ & Luminaires & 17 & 2 & 5 & 4.4 & 4 & 5 & 4 & 4.4 & 4.4 & 4.4 \\
\hline M Myer & MM64 & Internal & Content & \begin{tabular}{|l|} 
Recessed Slot \\
Lumianirs \\
parallel to desk \\
with compact \\
fluorescent wall- \\
washers (office)
\end{tabular} & & & & Luminaire $\mathrm{A}$ & $\begin{array}{l}\text { Instant-start should be } \\
\text { hyphentated }\end{array}$ & Luminaires & 17 & 2 & 5 & 4.4 & 4 & 5 & 4 & 4.4 & 4.4 & 4.4 \\
\hline \begin{tabular}{|l|} 
A Cortese \\
\end{tabular} & AC9 & Architect & General & & & & & & \begin{tabular}{|l|} 
The details on the various \\
vignettes are excellent. \\
However, I wonder if this is \\
the right word. I just don't \\
see your typical electrical \\
contractor using 'vignette'. \\
Maybe 'scenario'? OR \\
'strategy'?
\end{tabular} & Opinion & TBD & 1 & 2 & 1.8 & 1 & 2 & 2 & 1.7 & 1.8 & 1.8 \\
\hline E Smith & ES4 & Owner & & & & & & & $\begin{array}{l}\text { WRT the tenant, everything } \\
\text { is negotiable-- what will it } \\
\text { take to keep the tenant? To } \\
\text { compete? }\end{array}$ & Opinion & LT & 2 & 5 & 2.8 & 2 & 2 & 3 & 2.3 & 2.6 & 2.7 \\
\hline R Parish & RP5 & Owner & & & & & & & $\begin{array}{l}\text { Front end interface looks } \\
\text { great, easy to use, good } \\
\text { graphics. Now we need a } \\
\text { way to get it used by the } \\
\text { customer. }\end{array}$ & Opinion & LT & 2 & 5 & 4.4 & 2 & 2 & 3 & 2.3 & 3.6 & 4.1 \\
\hline R Parish & RP2 & Owner & & & & & & & $\begin{array}{l}\text { Would help to train CBRE } \\
\text { staff to be able to use the } \\
\text { tool. }\end{array}$ & Opinion & LT & 2 & 5 & 4.4 & 2 & 2 & 3 & 2.3 & 3.6 & 4.1 \\
\hline \begin{tabular}{|l|}
$J$ Manierre \\
\end{tabular} & JM1 & Utility & & & & & & \begin{tabular}{|l|} 
Jennifer Manierre, \\
NYSERDA
\end{tabular} & Can we add load profiles? & PDF & LT & 1 & 3 & 2.6 & 2 & 3 & 3 & 2.7 & 2.6 & 2.6 \\
\hline $\mid$\begin{tabular}{|l|} 
N Ferzacca \\
\end{tabular} & NF19 & Engineer & & & & & & & \begin{tabular}{|l|} 
Can we see where the \\
savings is coming from? \\
From the premium \\
equipment? Percentage \\
savings from occupancy \\
sensors? Maybe over time \\
we can learn more from the \\
data. There are problems \\
with giving too much \\
information also, can \\
sometimes only create \\
more questions. \\
\end{tabular} & PDF & 60 & 1 & 3 & 2.6 & 4 & 5 & 3 & 4.1 & 3.2 & 2.8 \\
\hline
\end{tabular}


Appendix E: Master Comments

\begin{tabular}{|c|c|c|c|c|c|c|c|c|c|c|c|c|c|c|c|c|c|c|c|c|}
\hline $\begin{array}{l}\text { Commenter } \\
\text { ID \# }\end{array}$ & $\begin{array}{l}\text { Comment } \\
\text { Reference } \\
\#\end{array}$ & $\begin{array}{l}\text { Commenter } \\
\text { Category }\end{array}$ & $\begin{array}{l}\text { Comment } \\
\text { Type }\end{array}$ & Space Type & $\mid \begin{array}{l}\text { Ceiling } \\
\text { Height// } \\
\text { Total Area }\end{array}$ & \begin{tabular}{|l|}
$\#$ \\
workstations/ \\
partition \\
height (open \\
office only) \\
\end{tabular} & $\left|\begin{array}{l}\text { Daylight } \\
\text { Input (if } \\
\text { applicable) }\end{array}\right|$ & $\begin{array}{l}\text { Reference webpage } \\
\text { or other location } \\
\text { (see } \\
\text { "Reference.doc") } \\
\end{array}$ & $\begin{array}{l}\text { Comment Description: } \\
\text { Issues, Suggestions \& } \\
\text { Requests }\end{array}$ & Action/Solution $\left[\begin{array}{l}S \\
0 \\
T\end{array}\right.$ & 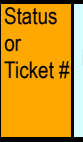 & $\begin{array}{c}\text { Frequenc } \\
\mathrm{y}(20 \%)\end{array}$ & $\begin{array}{c}\text { Importance } \\
\text { of comment } \\
(80 \%)\end{array}$ & \begin{tabular}{|} 
Sum \\
$(100 \%)$
\end{tabular} & $\begin{array}{c}\text { Ease of } \\
\text { Implementatio } \\
\mathrm{n}(30 \%)\end{array}$ & $\begin{array}{c}\text { Duration } \\
(40 \%)\end{array}$ & $\begin{array}{c}\text { Risk/ } \\
\text { Complicatio } \\
\mathrm{n}(30 \%)\end{array}$ & \begin{tabular}{|c|} 
Sum \\
$(100 \%)$
\end{tabular} & $\begin{array}{c}\begin{array}{c}\text { Short Term } \\
(40 \% \\
\text { Importance) } \\
(60 \% \text { Feasibility })\end{array} \\
\end{array}$ & $\begin{array}{c}\text { Long Term } \\
\text { (85\% } \\
\text { Importance) } \\
\text { (15\% Feasibility) }\end{array}$ \\
\hline C Dilouie & CD33 & Core Team & Content & & & & & & \begin{tabular}{l|} 
I found the open plan + \\
open plan perimeter control \\
guidance confusing. The \\
perimeter control plan was \\
presented first, the word \\
null appeared above the \\
control guidance drawing, \\
the system was labeled "12 \\
m," under the Performance \\
Specifications the intro \\
paragraph stopped mid \\
sentence, the text size \\
became smaller without \\
reason, the wrong control \\
guidance drawing was used \\
(with the wrong legend, the \\
subhead "Scheduling" was \\
made a bullet instead of a \\
bullet, and only scheduling \\
was presented, even \\
though there were two \\
options, one scheduling, \\
one vacancy sensing plus \\
one scheduling.
\end{tabular} & |PDF & 28 & 2 & 4 & 3.6 & 3 & 4 & 5 & 4 & 3.8 & 3.7 \\
\hline C Dilouie & CD26 & Core Team & General & & & & & & $\begin{array}{l}\text { At top of vignette summary } \\
\text { for the control part in the } \\
\text { vignette document, the } \\
\text { control option should be } \\
\text { identified. CLS needs to } \\
\text { change the impression it } \\
\text { gives of lighting controls, } \\
\text { which is an add-on to the } \\
\text { main event. Controls is the } \\
\text { main event. The control } \\
\text { method and equipment } \\
\text { used is just as important as } \\
\text { the choice of lamps, } \\
\text { ballasts and fixtures, and } \\
\text { where they are placed and } \\
\text { what they do. The overall } \\
\text { system is a lighting and } \\
\text { control system, and should } \\
\text { be identified as such } \\
\text { throughout. }\end{array}$ & PDF & 28 & 2 & 4 & 3.6 & 3 & 4 & 5 & 4 & 3.8 & 3.7 \\
\hline \begin{tabular}{|l|} 
C Dilouie \\
\end{tabular} & CD19 & & Content & & & & & & $\begin{array}{l}\text { Controls sections are } \\
\text { missing in the energy } \\
\text { summary document }\end{array}$ & PDF & 24 & 3 & 5 & 4.6 & 4 & 5 & 5 & 4.7 & 4.6 & 4.6 \\
\hline
\end{tabular}




\begin{tabular}{|c|c|c|c|c|c|c|c|c|c|c|c|c|c|c|c|c|c|c|c|c|}
\hline $\begin{array}{l}\text { Commenter } \\
\text { ID \# }\end{array}$ & \begin{tabular}{|l|} 
Comment \\
Reference \\
$\#$
\end{tabular} & $\begin{array}{l}\text { Commenter } \\
\text { Category }\end{array}$ & $\begin{array}{l}\text { Comment } \\
\text { Type }\end{array}$ & Space Type & \begin{tabular}{|l|} 
Ceiling \\
Height/ \\
Total Area
\end{tabular} & \begin{tabular}{|l}
$\#$ \\
workstations/ \\
partition \\
height (open \\
office only) \\
\end{tabular} & $\left|\begin{array}{l}\text { Daylight } \\
\text { Input (if } \\
\text { applicable) }\end{array}\right|$ & \begin{tabular}{|l|} 
Reference webpage \\
or other location \\
(see \\
"Reference.doc")
\end{tabular} & $\begin{array}{l}\text { Comment Description: } \\
\text { Issues, Suggestions \& } \\
\text { Requests }\end{array}$ & Action/Solution/ & \begin{tabular}{|l|}
$\begin{array}{l}\text { Status } \\
\text { or } \\
\text { Ticket \# }\end{array}$ \\
\end{tabular} & $\begin{array}{c}\text { Frequenc } \\
\mathrm{y}(20 \%)\end{array}$ & $\begin{array}{c}\text { Importance } \\
\text { of comment } \\
(80 \%)\end{array}$ & \begin{tabular}{|c|} 
Sum \\
$(100 \%)$
\end{tabular} & $\begin{array}{c}\text { Ease of } \\
\text { Implementatio } \\
\mathrm{n}(30 \%)\end{array}$ & \begin{tabular}{|c|} 
Duration \\
$(40 \%)$
\end{tabular} & $\begin{array}{c}\text { Risk/ } \\
\text { Complicatio } \\
\mathrm{n}(30 \%)\end{array}$ & \begin{tabular}{|c|} 
Sum \\
$(100 \%)$
\end{tabular} & \begin{tabular}{|c|} 
Short Term \\
$(40 \%$ \\
Importance) \\
$(60 \%$ Feasibility) \\
\end{tabular} & $\begin{array}{c}\text { Long Term } \\
(85 \% \\
\text { Importance) } \\
\text { (15\% Feasibility) }\end{array}$ \\
\hline D Maniccia & DM20 & Controls & & & & & & Energy Summary & \begin{tabular}{|l|} 
The energy summary says \\
retail office, fix the titles. \\
The controls selections are \\
not showing up on the \\
Energy Summary, it's \\
blank. The format for \\
showing controls selections \\
doesn't work, it's designed \\
to be an overall list. If you \\
are going to show which \\
controls are being used in \\
the Energy Summary, it \\
would need to be by space \\
type.
\end{tabular} & $\overline{P D F}$ & 24 & 3 & 5 & 4.6 & 4 & 5 & 5 & 4.7 & 4.6 & 4.6 \\
\hline J Yorgey & JY23 & Manufacturer & & & & & & Reports & $\begin{array}{l}\text { Not all data shows up on } \\
\text { energy summary page }\end{array}$ & PDF & 24 & 3 & 5 & 4.6 & 4 & 5 & 5 & 4.7 & 4.6 & 4.6 \\
\hline W Dau & WD7 & \begin{tabular}{|l|} 
Designer/IES \\
Chair
\end{tabular} & & & & & & & $\begin{array}{l}\text { Energy Summary controls } \\
\text { are not showing up in the } \\
\text { download packet. }\end{array}$ & PDF & 24 & 3 & 5 & 4.6 & 4 & 5 & 5 & 4.7 & 4.6 & 4.6 \\
\hline D Maniccia & DM19 & Controls & & & & & & Energy Summary & \begin{tabular}{|l|} 
Specify the kWh per each \\
vignette, not just in \\
summary at bottom.
\end{tabular} & PDF & 25 & 3 & 5 & 4.6 & 4 & 5 & 5 & 4.7 & 4.6 & 4.6 \\
\hline \begin{tabular}{|l} 
J Beutell \\
\end{tabular} & $\mid$ JB6 & Owner & & & & & & Energy Summary. & $\begin{array}{l}\text { Provide kWh per vignette } \\
\text { along with the total. Shows } \\
\text { where you are falling short } \\
\text { or using most of your } \\
\text { energy. }\end{array}$ & PDF & 25 & 3 & 5 & 4.6 & 4 & 5 & 5 & 4.7 & 4.6 & 4.6 \\
\hline P Lantine & PL3 & Utilities & & & & & & \begin{tabular}{|l|} 
Paul Lentine, \\
Northeast Utilities
\end{tabular} & $\begin{array}{l}\text { show kWh per vignette in } \\
\text { the Energy Summary. }\end{array}$ & PDF & 25 & 3 & 5 & 4.6 & 4 & 5 & 5 & 4.7 & 4.6 & 4.6 \\
\hline C DiLouie & CD18 & Core Team & \begin{tabular}{|l|} 
Download \\
Materials
\end{tabular} & & & & & & \begin{tabular}{|l} 
Project is identified as \\
"retail office"-should just \\
be identified as "office"
\end{tabular} & PDF & 27 & 3 & 5 & 4.6 & 4 & 5 & 5 & 4.7 & 4.6 & 4.6 \\
\hline J Yorgey & JY20 & Manufacturer & Functionality & & & & & \begin{tabular}{|l|}
$\begin{array}{l}\text { Energy saving } \\
\text { summary }\end{array}$ \\
\end{tabular} & No totals - page 4 blank & PDF & 27 & 3 & 5 & 4.6 & 4 & 5 & 5 & 4.7 & 4.6 & 4.6 \\
\hline N Ferzacca & NF17 & Engineer & & & & & & & $\begin{array}{l}\text { What is the basis for the } \\
\text { energy summary? } \\
\text { Occupancy hour summary, } \\
\text { etc. When this is divorced } \\
\text { from the webtool input } \\
\text { process, folks wouldn't } \\
\text { know the context. }\end{array}$ & PDF & 27 & 3 & 5 & 4.6 & 4 & 5 & 5 & 4.7 & 4.6 & 4.6 \\
\hline
\end{tabular}




\begin{tabular}{|c|c|c|c|c|c|c|c|c|c|c|c|c|c|c|c|c|c|c|c|c|}
\hline $\begin{array}{l}\text { Commenter } \\
\text { ID \# }\end{array}$ & $\begin{array}{l}\text { Comment } \\
\text { Reference } \\
\#\end{array}$ & $\begin{array}{l}\text { Commenter } \\
\text { Category }\end{array}$ & \begin{tabular}{|l} 
Comment \\
Type
\end{tabular} & Space Type & \begin{tabular}{|l|} 
Ceiling \\
Height/ \\
Total Area
\end{tabular} & \begin{tabular}{|l|}
$\#$ \\
workstations/ \\
partition \\
height (open \\
office only) \\
\end{tabular} & \begin{tabular}{|l} 
Daylight \\
Input (if \\
applicable)
\end{tabular} & \begin{tabular}{|l|} 
Reference webpage \\
or other location \\
(see \\
"Reference.doc")
\end{tabular} & $\begin{array}{l}\text { Comment Description: } \\
\text { Issues, Suggestions \& } \\
\text { Requests }\end{array}$ & Action/Solution & \begin{tabular}{|l|} 
Status \\
or \\
Ticket \#
\end{tabular} & $\begin{array}{c}\text { Frequenc } \\
\mathrm{y}(20 \%)\end{array}$ & $\begin{array}{c}\text { Importance } \\
\text { of comment } \\
(80 \%)\end{array}$ & \begin{tabular}{|c|} 
Sum \\
$(100 \%)$
\end{tabular} & $\begin{array}{c}\text { Ease of } \\
\text { Implementatio } \\
\mathrm{n}(30 \%)\end{array}$ & $\begin{array}{c}\text { Duration } \\
(40 \%)\end{array}$ & $\begin{array}{c}\text { Risk/ } \\
\text { Complicatio } \\
\mathrm{n}(30 \%)\end{array}$ & \begin{tabular}{|c|} 
Sum \\
$(100 \%)$
\end{tabular} \mid & 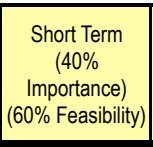 & $\begin{array}{c}\text { Long Term } \\
(85 \% \\
\text { Importance) } \\
\text { (15\% Feasibility) } \\
\end{array}$ \\
\hline C Dilouie & CD24 & Core Team & \begin{tabular}{|l|} 
Download \\
Materials
\end{tabular} & & & & & & $\begin{array}{l}\text { Open plan perimeter } \\
\text { lighting should follow the } \\
\text { open plan office for sense }\end{array}$ & PDF & 28 & 3 & 5 & 4.6 & 4 & 5 & 5 & 4.7 & 4.6 & 4.6 \\
\hline \begin{tabular}{|l|} 
C DiLouie \\
\end{tabular} & CD23 & Core Team & \begin{tabular}{|l|} 
Download \\
Materials
\end{tabular} & & & & & & $\begin{array}{l}\text { Controls section figure } \\
\text { should not say "Control } \\
\text { Section Figure" next to it, } \\
\text { make larger to fill to } \\
\text { margins }\end{array}$ & PDF & 29 & 3 & 5 & 4.6 & 4 & 5 & 5 & 4.7 & 4.6 & 4.6 \\
\hline C DiLouie & CD21 & Core Team & \begin{tabular}{|l|} 
Download \\
Materials
\end{tabular} & & & & & & \begin{tabular}{|l|} 
In the vignette summary, \\
control sections are \\
rendered poorly and \\
confusing: no subheads, \\
and zonal drawings and \\
implementation instructions \\
at the end, labeled in a way \\
that has no relevance to \\
the user \\
\end{tabular} & PDF & 29 & 3 & 5 & 4.6 & 4 & 5 & 5 & 4.7 & 4.6 & 4.6 \\
\hline \begin{tabular}{|l|} 
C DiLouie \\
\end{tabular} & CD27 & Core Team & \begin{tabular}{|l|} 
Download \\
Materials
\end{tabular} & & & & & & $\begin{array}{l}\text { Next to the cross section } \\
\text { drawing, "control section } \\
\text { figure" should be deleted, } \\
\text { then expand the drawing to } \\
\text { fill from margin to margin. }\end{array}$ & PDF & 29 & 3 & 5 & 4.6 & 4 & 5 & 5 & 4.7 & 4.6 & 4.6 \\
\hline C DiLouie & CD25 & Core Team & \begin{tabular}{|l|} 
Download \\
Materials
\end{tabular} & & & & & & \begin{tabular}{|l|} 
For the luminaire schedule \\
document, call it "project- \\
name_luminaire- \\
schedule.pdf"
\end{tabular} & PDF & 30 & 3 & 5 & 4.6 & 4 & 5 & 5 & 4.7 & 4.6 & 4.6 \\
\hline \begin{tabular}{|l|} 
C Dilouie \\
\end{tabular} & CD20 & Core Team & \begin{tabular}{|l|} 
Download \\
Materials
\end{tabular} & & & & & & $\begin{array}{l}\text { In the vignette summary, } \\
\text { change document name to } \\
\text { "project- } \\
\text { name_implementation.pdf" }\end{array}$ & PDF & 30 & 3 & 5 & 4.6 & 4 & 5 & 5 & 4.7 & 4.6 & 4.6 \\
\hline \begin{tabular}{|l|} 
C DiLouie \\
\end{tabular} & CD17 & Core Team & Functionality & & & & & & $\begin{array}{l}\text { In the energy summary } \\
\text { document, the document } \\
\text { dl's as } \\
\text { "implementation"-should } \\
\text { be named, "project- } \\
\text { name_energy- } \\
\text { summary.pdf" }\end{array}$ & PDF & 30 & 3 & 5 & 4.6 & 4 & 5 & 5 & 4.7 & 4.6 & 4.6 \\
\hline A Cortese & AC18 & Architect & \begin{tabular}{|l|} 
Download \\
Materials
\end{tabular} & & & & & & $\begin{array}{l}\text { The Luminarie Report } \\
\text { margins and layout seemed } \\
\text { off. I think the table needs } \\
\text { to be reformatted, but I } \\
\text { wasn't sure. }\end{array}$ & PDF & 31 & 3 & 5 & 4.6 & 4 & 5 & 5 & 4.7 & 4.6 & 4.6 \\
\hline
\end{tabular}




\begin{tabular}{|c|c|c|c|c|c|c|c|c|c|c|c|c|c|c|c|c|c|c|c|c|}
\hline $\begin{array}{l}\text { Commenter } \\
\text { ID \# }\end{array}$ & $\begin{array}{l}\text { Comment } \\
\text { Reference } \\
\#\end{array}$ & $\begin{array}{l}\text { Commenter } \\
\text { Category }\end{array}$ & \begin{tabular}{|l} 
Comment \\
Type
\end{tabular} & Space Type & \begin{tabular}{|l|} 
Ceiling \\
Height/ \\
Total Area
\end{tabular} & \begin{tabular}{|l|}
$\#$ \\
workstations/ \\
partition \\
height (open \\
office only) \\
\end{tabular} & $\left|\begin{array}{l}\text { Daylight } \\
\text { Input (if } \\
\text { applicable) }\end{array}\right|$ & $\begin{array}{l}\text { Reference webpage } \\
\text { or other location } \\
\text { (see } \\
\text { "Reference.doc") }\end{array}$ & $\begin{array}{l}\text { Comment Description: } \\
\text { Issues, Suggestions \& } \\
\text { Requests }\end{array}$ & Action/Solution/s & \begin{tabular}{|l|}
$\begin{array}{l}\text { Status } \\
\text { or } \\
\text { Ticket \# }\end{array}$ \\
\end{tabular} & $\begin{array}{c}\text { Frequenc } \\
\mathrm{y}(20 \%)\end{array}$ & $\begin{array}{c}\text { Importance } \\
\text { of comment } \\
(80 \%)\end{array}$ & \begin{tabular}{|c|} 
Sum \\
$(100 \%)$
\end{tabular} & $\begin{array}{c}\text { Ease of } \\
\text { Implementatio } \\
\mathrm{n}(30 \%)\end{array}$ & \begin{tabular}{|c|} 
Duration \\
$(40 \%)$
\end{tabular} & $\begin{array}{c}\text { Risk/ } \\
\text { Complicatio } \\
\mathrm{n}(30 \%)\end{array}$ & \begin{tabular}{|} 
Sum \\
$(100 \%)$
\end{tabular} \mid & \begin{tabular}{|c|} 
Short Term \\
$(40 \%$ \\
Importance) \\
$(60 \%$ Feasibility) \\
\end{tabular} & $\begin{array}{c}\text { Long Term } \\
(85 \% \\
\text { Importance) } \\
\text { (15\% Feasibility) }\end{array}$ \\
\hline K Cota & $\overline{K C 6}$ & Utility & & & & & & $\begin{array}{l}\begin{array}{l}\text { Kelly Cota, National } \\
\text { Grid }\end{array} \\
\end{array}$ & $\begin{array}{l}\text { Can the lamp field be a } \\
\text { drop down menu? }\end{array}$ & Phase 2 & TBD & 1 & 2 & 1.8 & 3 & 4 & 3 & 3.4 & 2.4 & 2.0 \\
\hline N Ferzacca & NF23 & Engineer & & & & & & & \begin{tabular}{|l|} 
The time involved in \\
building an entire building \\
would be extensive. What \\
about collaborating with \\
ComCheck? Provide an \\
ouptut that can be imported \\
into ComCheck.
\end{tabular} & Phase 2 & LT & 1 & 3 & 2.6 & 2 & 2 & 3 & 2.3 & 2.5 & 2.6 \\
\hline N Ferzacca & NF24 & Engineer & & & & & & & $\begin{array}{l}\text { Does the amount of money } \\
\text { do enough to get you past } \\
\text { the hassle factor? Maybe } \\
\text { yes, sometimes. Takeoffs } \\
\text { can be perfect for junior } \\
\text { employees. Larger offices } \\
\text { have all levels of } \\
\text { employees to be able to do } \\
\text { that, smaller offices, not so } \\
\text { much. }\end{array}$ & Phase 2 & LT & 2 & 5 & 2.8 & 2 & 2 & 3 & 2.3 & 2.6 & 2.7 \\
\hline K Cota & KC3 & Utility & & & & & & $\begin{array}{l}\text { Kelly Cota, National } \\
\text { Grid }\end{array}$ & $\begin{array}{l}\text { LEED credits don't allow for } \\
\text { kWh, so we need to also } \\
\text { show the LEED savings, } \\
\text { percentage of savings in } \\
\text { w/SF. }\end{array}$ & Phase 2 & 34 & 1 & 3 & 2.6 & 4 & 4 & 4 & 4 & 3.2 & 2.8 \\
\hline P Lantine & PL4 & Utilities & & & & & & \begin{tabular}{|l|} 
Paul Lentine, \\
Northeast Utilities
\end{tabular} & $\begin{array}{l}\text { Can you put in a notes field } \\
\text { to flag equipment that isn't } \\
\text { high performance? }\end{array}$ & Phase 2 & 62 & 2 & 4 & 3.6 & 3 & 3 & 2 & 2.7 & 3.2 & 3.5 \\
\hline \begin{tabular}{|l|} 
D Maniccia \\
\end{tabular} & DM18 & Controls & & & & & & Energy Summary & \begin{tabular}{|l|} 
\\
Once again, in the energy \\
summary you need to \\
specify individual room \\
sizes and quantity of \\
rooms.
\end{tabular} & Phase 2 & 32 & 2 & 4 & 3.6 & 4 & 5 & 5 & 4.7 & 4.0 & 3.8 \\
\hline J Yorgey & JY15 & Manufacturer & Functionality & & & & & & \begin{tabular}{|l|} 
Private office summary did \\
not have correct \# of rooms \\
or space name, also \\
incorrect ceiling ht
\end{tabular} & Phase 2 & 32 & 2 & 4 & 3.6 & 4 & 5 & 5 & 4.7 & 4.0 & 3.8 \\
\hline
\end{tabular}




\begin{tabular}{|c|c|c|c|c|c|c|c|c|c|c|c|c|c|c|c|c|c|c|c|c|}
\hline $\begin{array}{l}\text { Commenter } \\
\text { ID \# }\end{array}$ & \begin{tabular}{|l|} 
Comment \\
Reference \\
$\#$
\end{tabular} & $\begin{array}{l}\text { Commenter } \\
\text { Category }\end{array}$ & \begin{tabular}{|l} 
Comment \\
Type
\end{tabular} & Space Type & \begin{tabular}{|l|} 
Ceiling \\
Height/ \\
Total Area
\end{tabular} & \begin{tabular}{|l|}
$\#$ \\
workstations/ \\
partition \\
height (open \\
office only) \\
\end{tabular} & $\left|\begin{array}{|}\text { Daylight } \\
\text { Input (if } \\
\text { applicable) }\end{array}\right|$ & \begin{tabular}{|l|} 
Reference webpage \\
or other location \\
(see \\
"Reference.doc")
\end{tabular} & $\begin{array}{l}\text { Comment Description: } \\
\text { Issues, Suggestions \& } \\
\text { Requests }\end{array}$ & Action/Solution & \begin{tabular}{|l|} 
Status \\
or \\
Ticket \#
\end{tabular} & $\begin{array}{c}\text { Frequenc } \\
\mathrm{y}(20 \%)\end{array}$ & $\begin{array}{c}\text { Importance } \\
\text { of comment } \\
(80 \%)\end{array}$ & \begin{tabular}{|c|} 
Sum \\
$(100 \%)$
\end{tabular} & $\begin{array}{c}\text { Ease of } \\
\text { Implementatio } \\
\mathrm{n}(30 \%)\end{array}$ & \begin{tabular}{|c|} 
Duration \\
$(40 \%)$
\end{tabular} & $\begin{array}{c}\text { Risk/ } \\
\text { Complicatio } \\
\mathrm{n}(30 \%)\end{array}$ & \begin{tabular}{|} 
Sum \\
$(100 \%)$
\end{tabular} \mid & 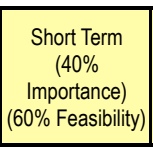 & $\begin{array}{c}\text { Long Term } \\
(85 \% \\
\text { Importance) } \\
\text { (15\% Feasibility) }\end{array}$ \\
\hline K Cota & KC4 & Utility & & & & & & $\begin{array}{l}\begin{array}{l}\text { Kelly Cota, National } \\
\text { Grid }\end{array} \\
\end{array}$ & $\begin{array}{l}\text { Why is there zero SF for } \\
\text { wallwashing? Maybe it } \\
\text { should show N/A or linear } \\
\text { footage }\end{array}$ & Phase 2 & 35 & 2 & 5 & 4.4 & 3 & 4 & 4 & 3.7 & 4.1 & 4.3 \\
\hline J Yorgey & JY3 & Manufacturer & Functionality & & & & & \begin{tabular}{|l|} 
Project description \\
energy estimate
\end{tabular} & $\begin{array}{l}\text { A explorer message pops } \\
\text { up "No } \\
\text { controlCombSavings found } \\
\text { with pace ID: } 28 \text { energy } \\
\text { code CEZ_IECC2006" }\end{array}$ & Phase 2 & 33 & 1 & 5 & 4.2 & 4 & 4 & 5 & 4.3 & 4.2 & 4.2 \\
\hline HMcKay & HM25 & Designer & \begin{tabular}{|l|} 
Ease of \\
Use/Flow
\end{tabular} & & & & & & $\begin{array}{l}\text { The "Control" tab does not } \\
\text { seem to be very } \\
\text { informative. It is so basic, it } \\
\text { should be the first tab, as } \\
\text { an introduction. }\end{array}$ & \begin{tabular}{|l|} 
Presentation \\
Order
\end{tabular} & TBD & 1 & 2 & 1.8 & 2 & 3 & 2 & 2.4 & 2.0 & 1.9 \\
\hline HMcKay & HM22 & Designer & Content & & & & & & $\begin{array}{l}\text { Control templates should } \\
\text { be presented in order of } \\
\text { relative energy savings, } \\
\text { and with brief explanation, } \\
\text { before going to detail } \\
\text { pages. }\end{array}$ & \begin{tabular}{|l|}
$\begin{array}{l}\text { Presentation } \\
\text { Order }\end{array}$ \\
\end{tabular} & TBD & 1 & 3 & 2.6 & 3 & 3 & 3 & 3 & 2.8 & 2.7 \\
\hline P Lantine & PL2 & Utilities & & & & & & \begin{tabular}{|l} 
Paul Lentine, \\
Northeast Utilities
\end{tabular} & $\begin{array}{l}\text { do we want to choose the } \\
\text { order of presentation of the } \\
\text { vignetes? Prioritize by } \\
\text { LPD? }\end{array}$ & \begin{tabular}{|l|} 
Presentation \\
Order
\end{tabular} & 37 & 2 & 4 & 3.6 & 4 & 3 & 4 & 3.6 & 3.6 & 3.6 \\
\hline HMcKay & HM16 & Designer & Content & & & & & & $\begin{array}{l}\text { No tab for interior design } \\
\text { layouts or strategies. The } \\
\text { user should know this is the } \\
\text { least efficient layout before } \\
\text { proceeding. Information is } \\
\text { useless at the report } \\
\text { printout stage. }\end{array}$ & \begin{tabular}{|l|} 
Presentation \\
Order
\end{tabular} & 36 & 2 & 4 & 3.6 & 4 & 4 & 3 & 3.7 & 3.6 & 3.6 \\
\hline C DiLouie & CD16 & Core Team & Content & & & & & & \begin{tabular}{|l|} 
Open office options, \\
daylight and no daylight, \\
have no digital control \\
option, it's not showing up \\
\end{tabular} & \begin{tabular}{|l|}
$\begin{array}{l}\text { Presentation } \\
\text { Order }\end{array}$ \\
\end{tabular} & 5 & 2 & 5 & 4.4 & 4 & 3 & 4 & 3.6 & 4.1 & 4.3 \\
\hline N Miller & NM19 & Internal & & & & & & & $\begin{array}{l}\text { Do you think it's important } \\
\text { to mention the aiming angle } \\
\text { of the light so that there is } \\
\text { not a serious shadow cast } \\
\text { from one shelf onto the } \\
\text { merchandise below it? }\end{array}$ & Retail & 39 & 1 & 3 & 2.6 & 2 & 3 & 3 & 2.7 & 2.6 & 2.6 \\
\hline
\end{tabular}




\begin{tabular}{|c|c|c|c|c|c|c|c|c|c|c|c|c|c|c|c|c|c|c|c|c|}
\hline $\begin{array}{l}\text { Commenter } \\
\text { ID\# \# }\end{array}$ & $\begin{array}{l}\text { Comment } \\
\text { Reference } \\
\#\end{array}$ & $\begin{array}{l}\text { Commenter } \\
\text { Category }\end{array}$ & $\begin{array}{l}\text { Comment } \\
\text { Type }\end{array}$ & Space Type & \begin{tabular}{|l|} 
Ceiling \\
Height/ \\
Total Area
\end{tabular} & \begin{tabular}{|l|}
$\#$ \\
workstations/ \\
partition \\
height (open \\
office only) \\
\end{tabular} & $\left|\begin{array}{l}\text { Daylight } \\
\text { Input (if } \\
\text { applicable) }\end{array}\right|$ & 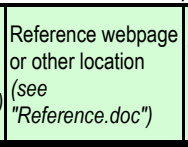 & $\begin{array}{l}\text { Comment Description: } \\
\text { Issues, Suggestions \& } \\
\text { Requests }\end{array}$ & Action/Solution/ & \begin{tabular}{|l|} 
Status \\
or \\
Ticket \#
\end{tabular} & \begin{tabular}{|c|} 
Frequenc \\
$\mathrm{y}(20 \%)$
\end{tabular} & $\begin{array}{c}\text { Importance } \\
\text { of comment } \\
(80 \%)\end{array}$ & $\begin{array}{c}\text { Sum } \\
(100 \%)\end{array}$ & $\begin{array}{c}\text { Ease of } \\
\text { Implementatio } \\
\mathrm{n}(30 \%)\end{array}$ & \begin{tabular}{|c|} 
Duration \\
$(40 \%)$
\end{tabular} & $\begin{array}{c}\text { Risk/ } \\
\text { Complicatio } \\
\mathrm{n}(30 \%)\end{array}$ & \begin{tabular}{|} 
Sum \\
$(100 \%)$
\end{tabular} \mid & 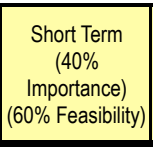 & $\begin{array}{c}\text { Long Term } \\
(85 \% \\
\text { Importance) } \\
\text { (15\% Feasibility) } \\
\end{array}$ \\
\hline N Miller & NM1 & 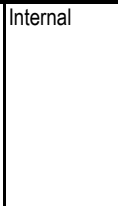 & Content & Pharmacy & & & & & $\begin{array}{l}\text { I expected to find more } \\
\text { types of areas available, } \\
\text { like the Pharmacy area, or } \\
\text { the stockroom, or the } \\
\text { checkout desk. }\end{array}$ & Retail & TBD & 1 & 3 & 2.6 & 3 & 2 & 4 & 2.9 & 2.7 & 2.6 \\
\hline K Abernathy & KA10 & Designer & General & Pharmacy & & & & & \begin{tabular}{|} 
I could not figure out what \\
my ceiling height was did I \\
miss something.
\end{tabular} & Retail & 45 & 2 & 4 & 3.6 & 2 & 2 & 3 & 2.3 & 3.1 & 3.4 \\
\hline N Miller & NM10 & Internal & General & \begin{tabular}{|l|} 
Pharmacy \\
Design \\
Vignettes
\end{tabular} & & & & \begin{tabular}{|l|}
$\begin{array}{l}\text { Perimeter - parallel } \\
\text { to walls }\end{array}$ \\
\end{tabular} & \begin{tabular}{|l|} 
assume the W/sf value is \\
multiplied against the \\
square footage in that \\
perimeter aisle, not the full \\
store. Is there an \\
explanation somewhere on \\
this? And, it's not clear to \\
me that the lighting for the \\
perimeter is also lighting \\
the gondola shelves \\
immediately opposite the \\
outside wall. I think it's \\
important to point that out, \\
or else another linear row \\
of lighting would be \\
needed.
\end{tabular} & Retail & 41 & 1 & 4 & 3.4 & 4 & 4 & 4 & 4 & 3.6 & 3.5 \\
\hline N Miller & NM11 & Internal & Content & \begin{tabular}{|l}
$\begin{array}{l}\text { Pharmacy Des } \\
\text { Vignettes }\end{array}$ \\
\end{tabular} & & & & \begin{tabular}{|l|} 
Center aisle \\
Continuous row of \\
luminaires
\end{tabular} & $\begin{array}{l}\text { There's no explanation of } \\
\text { why the luminaires are off- } \\
\text { center. How does this do } \\
\text { for shoppers that are on the } \\
\text { other side of the bins, either } \\
\text { looking at the unlit end- } \\
\text { caps or seeing into the } \\
\text { bins? }\end{array}$ & Retail & 41 & 1 & 4 & 3.4 & 4 & 4 & 4 & 4 & 3.6 & 3.5 \\
\hline N Miller & NM4 & Internal & Content & \begin{tabular}{|l|} 
Pharm - Genl \\
Merch
\end{tabular} & & & & \begin{tabular}{|l} 
Strategy-parallel \\
spacing-dayltg
\end{tabular} & \begin{tabular}{|l|} 
End of first paragraph has \\
incomplete sentence. So \\
does the end of the \\
Luminaires section. \\
\end{tabular} & Retail & 41 & 1 & 4 & 3.4 & 4 & 4 & 4 & 4 & 3.6 & 3.5 \\
\hline N Miller & NM5.2 & Internal & Content & \begin{tabular}{|l|} 
Pharm - Genl \\
Merch
\end{tabular} & & & & & \begin{tabular}{|l|} 
Occupancy sensors: \\
"During low activity times..." \\
Do you mean after hours? \\
It would be an unusual \\
store that would condone \\
shutting off lights in a \\
section of store before \\
closing hours. It would \\
keep the customers away, \\
and they wouldn't be able \\
to see signage in the area.
\end{tabular} & Retail & 41 & 1 & 4 & 3.4 & 4 & 4 & 4 & 4 & 3.6 & 3.5 \\
\hline N Miller & NM2 & Internal & Content & $\begin{array}{l}\text { Pharmacy - } \\
\text { Gen'l Merch }\end{array}$ & & & & & $\begin{array}{l}\text { Horiz illum. Is mentioned, } \\
\text { but is it measured at 30", } \\
0^{0 ",} \text { or 3"? How about } \\
\text { vertical illuminance on the } \\
\text { gondola shelves? }\end{array}$ & Retail & 40 & 2 & 4 & 3.6 & 4 & 4 & 4 & 4 & 3.8 & 3.7 \\
\hline
\end{tabular}




\begin{tabular}{|c|c|c|c|c|c|c|c|c|c|c|c|c|c|c|c|c|c|c|c|c|}
\hline $\begin{array}{l}\text { Commenter } \\
\text { ID \# }\end{array}$ & \begin{tabular}{|l|} 
Comment \\
Reference \\
$\#$
\end{tabular} & $\begin{array}{l}\text { Commenter } \\
\text { Category }\end{array}$ & $\begin{array}{l}\text { Comment } \\
\text { Type }\end{array}$ & Space Type & \begin{tabular}{|l|} 
Ceiling \\
Height/ \\
Total Area
\end{tabular} & \begin{tabular}{|l|}
$\#$ \\
workstations/ \\
partition \\
height (open \\
office only) \\
\end{tabular} & $\left|\begin{array}{l}\text { Daylight } \\
\text { Input (if } \\
\text { applicable) }\end{array}\right|$ & $\begin{array}{l}\text { Reference webpage } \\
\text { or other location } \\
\text { (see } \\
\text { "Reference.doc") } \\
\end{array}$ & $\begin{array}{l}\text { Comment Description: } \\
\text { Issues, Suggestions \& } \\
\text { Requests }\end{array}$ & Action/Solution $\left[\begin{array}{l}S \\
\text { or } \\
\mathrm{Ti}\end{array}\right.$ & $\begin{array}{l}\text { Status } \\
\text { or } \\
\text { Ticket \# }\end{array}$ & $\begin{array}{c}\text { Frequenc } \\
\mathrm{y}(20 \%)\end{array}$ & $\begin{array}{c}\text { Importance } \\
\text { of comment } \\
(80 \%)\end{array}$ & $\begin{array}{c}\text { Sum } \\
(100 \%)\end{array}$ & $\begin{array}{c}\text { Ease of } \\
\text { Implementatio } \\
\mathrm{n}(30 \%)\end{array}$ & $\begin{array}{c}\text { Duration } \\
(40 \%)\end{array}$ & $\begin{array}{c}\text { Risk/ } \\
\text { Complicatio } \\
\mathrm{n}(30 \%)\end{array}$ & $\begin{array}{c}\text { Sum } \\
(100 \%)\end{array}$ & \begin{tabular}{|c|} 
Short Term \\
$(40 \%$ \\
Importance) \\
(60\% Feasibility)
\end{tabular} & $\begin{array}{c}\text { Long Term } \\
(85 \% \\
\text { Importance) } \\
\text { (15\% Feasibility) } \\
\end{array}$ \\
\hline N Miller & NM9.2 & Internal & & & & & & & \begin{tabular}{|l|} 
I would add vertical \\
illuminance on the \\
merchandise shelves to be \\
a high priority, more than \\
the horizontal illuminance.
\end{tabular} & Retail & 40 & 2 & 4 & 3.6 & 4 & 4 & 4 & 4 & 3.8 & 3.7 \\
\hline N Miller & NM12 & Internal & & & & & & & \begin{tabular}{|l|} 
I am concerned that the \\
general merchandise aisles \\
use 2lamp T8 fixtures to \\
light the displays, but the \\
perimeter aisle light uses a \\
1 lamp T8 fixture. Maybe \\
I'm getting this wrong.
\end{tabular} & Retail & 39 & 2 & 5 & 4.4 & 4 & 4 & 4 & 4 & 4.2 & 4.3 \\
\hline KAbernathy & KA3 & Designer & General & Pharmacy & & & & \begin{tabular}{|l|} 
Luminaires Parallel \\
to Wall with \\
Skylights Pharmacy \\
under Luminaires
\end{tabular} & $\begin{array}{l}\text { I am unable to bring in the } \\
\text { photos. }\end{array}$ & Retail & 42 & 2 & 5 & 4.4 & 5 & 5 & 5 & 5 & 4.6 & 4.5 \\
\hline KAbernathy & KA7 & Designer & General & Pharmacy & & & & \begin{tabular}{|l|} 
Luminaires Parallel \\
to Wall Pharmacy - \\
under Luminaires
\end{tabular} & $\begin{array}{l}\text { I am unable to bring in the } \\
\text { photos. }\end{array}$ & Retail & 42 & 2 & 5 & 4.4 & 5 & 5 & 5 & 5 & 4.6 & 4.5 \\
\hline N Miller & NM15 & Internal & & & & & & & the photos will not come in & Retail & 42 & 2 & 5 & 4.4 & 5 & 5 & 5 & 5 & 4.6 & 4.5 \\
\hline W Dau & WD2 & \begin{tabular}{|l|}
$\begin{array}{l}\text { Designer/IES } \\
\text { Chair }\end{array}$ \\
\end{tabular} & & & & & & & $\begin{array}{l}\text { Is there a way to select all } \\
\text { of the spaces that will apply } \\
\text { ahead of time, first, rather } \\
\text { than going back and forth? }\end{array}$ & UI & TBD & 1 & 2 & 1.8 & 1 & 2 & 2 & 1.7 & 1.8 & 1.8 \\
\hline M Dare & MD2 & Utility & & & & & & & \begin{tabular}{|l|} 
As I was building the open \\
plan space, I ended up \\
typing private office info (I \\
think I got distracted by the \\
example of "e.g., \\
Executive Office." Would it \\
let me do go through and \\
do this wrong?
\end{tabular} & UI & TBD & 1 & 2 & 1.8 & 3 & 2 & 3 & 2.6 & 2.1 & 1.9 \\
\hline N Miller & NM3 & Internal & \begin{tabular}{|l|} 
Functionality \\
\end{tabular} & $\begin{array}{l}\text { Pharmacy - } \\
\text { Gen'l Merch }\end{array}$ & & & & & $\begin{array}{l}\text { Couldn't open the } \\
\text { photometric distribution } \\
\text { icon when I clicked on it. }\end{array}$ & UI & TBD & 1 & 2 & 1.8 & 3 & 2 & 3 & 2.6 & 2.1 & 1.9 \\
\hline
\end{tabular}




\begin{tabular}{|c|c|c|c|c|c|c|c|c|c|c|c|c|c|c|c|c|c|c|c|c|}
\hline $\begin{array}{l}\text { Commenter } \\
\text { ID \# }\end{array}$ & $\begin{array}{l}\text { Comment } \\
\text { Reference } \\
\#\end{array}$ & $\begin{array}{l}\text { Commenter } \\
\text { Category }\end{array}$ & $\begin{array}{l}\text { Comment } \\
\text { Type }\end{array}$ & Space Type & \begin{tabular}{|l|} 
Ceiling \\
Height/ \\
Total Area
\end{tabular} & \begin{tabular}{|l}
$\#$ \\
workstations/ \\
partition \\
height (open \\
office only) \\
\end{tabular} & $\left|\begin{array}{l}\text { Daylight } \\
\text { Input (if } \\
\text { applicable) }\end{array}\right|$ & \begin{tabular}{|l|} 
Reference webpage \\
or other location \\
(see \\
"Reference.doc")
\end{tabular} & $\begin{array}{l}\text { Comment Description: } \\
\text { Issues, Suggestions \& } \\
\text { Requests }\end{array}$ & \begin{tabular}{|l} 
Action/Solution \\
\\
or \\
or \\
Tit \\
\end{tabular} & $\begin{array}{l}\text { Status } \\
\text { or } \\
\text { Ticket \# }\end{array}$ & $\begin{array}{c}\text { Frequenc } \\
\mathrm{y}(20 \%)\end{array}$ & $\begin{array}{c}\text { Importance } \\
\text { of comment } \\
(80 \%)\end{array}$ & $\begin{array}{c}\text { Sum } \\
(100 \%)\end{array}$ & $\begin{array}{c}\text { Ease of } \\
\text { Implementatio } \\
\mathrm{n}(30 \%)\end{array}$ & $\begin{array}{c}\text { Duration } \\
(40 \%)\end{array}$ & $\begin{array}{c}\text { Risk/ } \\
\text { Complicatio } \\
\mathrm{n}(30 \%)\end{array}$ & \begin{tabular}{|c|} 
Sum \\
$(100 \%)$
\end{tabular} & \begin{tabular}{|c|} 
Short Term \\
$(40 \%$ \\
Importance) \\
$(60 \%$ Feasibility) \\
\end{tabular} & $\begin{array}{c}\text { Long Term } \\
(85 \% \\
\text { Importance) } \\
\text { (15\% Feasibility) }\end{array}$ \\
\hline M Dare & $\overline{M D 3}$ & Utility & & & & & & & $\begin{array}{l}\text { Why is it asking me how } \\
\text { many rooms there are in an } \\
\text { open plan? Open plan is } \\
\text { always } 1 \text { room. }\end{array}$ & $\overline{\mathrm{UI}}$ & TBD & 1 & 2 & 1.8 & 3 & 4 & 2 & 3.1 & 2.3 & 2.0 \\
\hline J Yorgey & JY13 & Manufacturer & \begin{tabular}{|l|} 
Ease of \\
Use/Flow
\end{tabular} & & & & & $\begin{array}{l}\text { Space type } \\
\text { summary }\end{array}$ & $\begin{array}{l}\text { ceiling ht - use pull down } \\
\text { with multiple choice }\end{array}$ & UI & 5 & 1 & 2 & 1.8 & 4 & 3 & 3 & 3.3 & 2.4 & 2.0 \\
\hline J Yorgey & JY22 & Manufacturer & \begin{tabular}{|l|} 
Appearancel \\
Aesthetics
\end{tabular} & & & & & Reports & $\begin{array}{l}\text { "download" label should be } \\
\text { called "reports" }\end{array}$ & UI & 30 & 1 & 2 & 1.8 & 4 & 3 & 3 & 3.3 & 2.4 & 2.0 \\
\hline D Maniccia & DM15 & Controls & & & & & & & \begin{tabular}{|l|} 
Would it make sense to \\
show the percentage of \\
savings from LPD vs. \\
controls by having two \\
colors in the energy \\
savings bar? Or, is that an \\
enhancement for a next \\
version, once there is data \\
to look at?
\end{tabular} & UI & 60 & 1 & 3 & 2.6 & 2 & 2 & 3 & 2.3 & 2.5 & 2.6 \\
\hline HMcKay & HM8 & Designer & Content & & & & & & \begin{tabular}{|l|} 
"Perimeter open-plan" is \\
very confusing. Menu plan \\
makes it look like it has a \\
width in plan. Since user is \\
forced to define open plan \\
first, they might deduct \\
some square footage from \\
the open plan area. Users \\
may also assume it refers \\
to the perimeter windows, \\
or \\
maybe a daylighting zone, \\
or perimeter circulation \\
paths. Why not call it "Open \\
Plan Wall washing".
\end{tabular} & UI & 15 & 1 & 3 & 2.6 & 4 & 4 & 2 & 3.4 & 2.9 & 2.7 \\
\hline M Myer & MM633 & $\mid$ Internal & Content & \begin{tabular}{|l|} 
Add Open Plan \\
Space-Controls \\
Options
\end{tabular} & & & & & $\begin{array}{l}\text { Recommend changing the } \\
\text { heading of this window to } \\
\text { say controls }\end{array}$ & UI & 57 & 1 & 3 & 2.6 & 4 & 4 & 4 & 4 & 3.2 & 2.8 \\
\hline M Myer & MM631 & Internal & Content & \begin{tabular}{|l|} 
Add Open Plan \\
Space- \\
Daylighting \\
questions
\end{tabular} & & & & & $\begin{array}{l}\text { Recommend changing the } \\
\text { heading of this window to } \\
\text { say daylighting }\end{array}$ & UI & 57 & 1 & 3 & 2.6 & 4 & 4 & 4 & 4 & 3.2 & 2.8 \\
\hline
\end{tabular}


Appendix E: Master Comments List

\begin{tabular}{|c|c|c|c|c|c|c|c|c|c|c|c|c|c|c|c|c|c|c|c|}
\hline $\begin{array}{l}\text { Commenter } \\
\text { ID \# }\end{array}$ & \begin{tabular}{|l|} 
Comment \\
Reference \\
$\#$
\end{tabular} & $\begin{array}{l}\text { Commenter } \\
\text { Category }\end{array}$ & \begin{tabular}{|l} 
Comment \\
Type
\end{tabular} & Space Type & \begin{tabular}{|l|} 
Ceiling \\
Height/ \\
Total Area
\end{tabular} & \begin{tabular}{|l|}
$\#$ \\
workstations/ \\
partition \\
height (open \\
office only) \\
\end{tabular} & \begin{tabular}{|l|} 
Daylight \\
Input (if \\
applicable)
\end{tabular} & \begin{tabular}{|l|} 
Reference webpage \\
or other location \\
(see \\
"Reference.doc")
\end{tabular} & $\begin{array}{l}\text { Comment Description: } \\
\text { Issues, Suggestions \& } \\
\text { Requests }\end{array}$ & 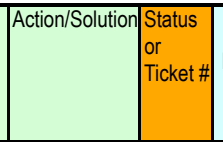 & $\begin{array}{c}\text { Frequenc } \\
y(20 \%)\end{array}$ & $\begin{array}{c}\text { Importance } \\
\text { of comment } \\
(80 \%)\end{array}$ & $\begin{array}{c}\text { Sum } \\
(100 \%)\end{array}$ & $\begin{array}{c}\text { Ease of } \\
\text { Implementatio } \\
\mathrm{n}(30 \%)\end{array}$ & $\begin{array}{c}\text { Duration } \\
(40 \%)\end{array}$ & $\begin{array}{c}\text { Risk/ } \\
\text { Complicatio } \\
\mathrm{n}(30 \%)\end{array}$ & \begin{tabular}{|} 
Sum \\
$(100 \%)$
\end{tabular} & \begin{tabular}{|c|} 
Short Term \\
$(40 \%$ \\
Importance) \\
(60\% Feasibility)
\end{tabular} & $\begin{array}{c}\text { Long Term } \\
(85 \% \\
\text { Importance) } \\
\text { (15\% Feasibility }\end{array}$ \\
\hline C DiLouie & CD13 & Core Team & $\begin{array}{l}\text { Appearancel } \\
\text { Aesthetics }\end{array}$ & & & & & & $\begin{array}{l}\text { After finishing, I saw a one- } \\
\text { line description of what I } \\
\text { chose, the lighting I chose } \\
\text { was spelled out, but the } \\
\text { controls were shown as } \\
\text { symbols-why symbols? } \\
\text { Plus symbols aren't legible. } \\
\text { Suggest spelling out the } \\
\text { controls, as they are part of } \\
\text { the lighting system }\end{array}$ & UI & 1 & 3 & 2.6 & 4 & 5 & 3 & 4.1 & 3.2 & 2.8 \\
\hline D Maniccia & DM3 & Controls & & & & & & & $\begin{array}{l}\text { Instead of having me do } \\
\text { the math for the total area, } \\
\text { can you put in SF per each } \\
\text { of private offices? }\end{array}$ & UI & 2 & 4 & 3.6 & 3 & 3 & 4 & 3.3 & 3.5 & 3.6 \\
\hline \begin{tabular}{|l} 
D Maniccia \\
\end{tabular} & DM13 & Controls & & & & & & & $\begin{array}{l}\text { The finish screen that } \\
\text { allows me to confirm the } \\
\text { info also needs to change } \\
\text { so that I can see the size of } \\
\text { the office, and the quantity } \\
\text { of each of those offices, } \\
\text { rather than the oveall } \\
\text { square footage (which } \\
\text { could so easily be wrong, } \\
\text { that's a back of the } \\
\text { envelope calculation.) }\end{array}$ & UI & 2 & 4 & 3.6 & 3 & 3 & 4 & 3.3 & 3.5 & 3.6 \\
\hline J Yorgey & JY12 & Manufacturer & \begin{tabular}{|l} 
Ease of \\
Use/Flow
\end{tabular} & & & & & $\begin{array}{l}\text { Space type } \\
\text { summary }\end{array}$ & $\begin{array}{l}\text { have ability to but in } \\
\text { demensions and have } \\
\text { program calculate sq ft }\end{array}$ & UI & 2 & 4 & 3.6 & 3 & 3 & 4 & 3.3 & 3.5 & 3.6 \\
\hline \begin{tabular}{|l|} 
N Ferzacca \\
\end{tabular} & \begin{tabular}{|l|l|} 
NF18 \\
\end{tabular} & Engineer & & & & & & & $\begin{array}{l}\text { Should we have a warning } \\
\text { about going backwards } \\
\text { after the downloads have } \\
\text { been done (sort of like the } \\
\text { messages you see for } \\
\text { resending forms)? }\end{array}$ & UI & 2 & 4 & 3.6 & 3 & 3 & 4 & 3.3 & 3.5 & 3.6 \\
\hline J Yorgey & JY4 & Manufacturer & Functionality & & & & & $\begin{array}{l}\text { Project description } \\
\text { energy estimate }\end{array}$ & $\begin{array}{l}\text { Add ability to include } \\
\text { weekend hours }\end{array}$ & 49 & 2 & 4 & 3.6 & 3 & 4 & 4 & 3.7 & 3.6 & 3.6 \\
\hline
\end{tabular}




\begin{tabular}{|c|c|c|c|c|c|c|c|c|c|c|c|c|c|c|c|c|c|c|c|c|}
\hline $\begin{array}{l}\text { Commenter } \\
\text { ID \# }\end{array}$ & $\begin{array}{l}\text { Comment } \\
\text { Reference } \\
\#\end{array}$ & $\begin{array}{l}\text { Commenter } \\
\text { Category }\end{array}$ & \begin{tabular}{|l} 
Comment \\
Type
\end{tabular} & Space Type & \begin{tabular}{|l|} 
Ceiling \\
Height/ \\
Total Area
\end{tabular} & $\begin{array}{l}\# \\
\text { workstations/ } \\
\text { partition } \\
\text { height (open } \\
\text { office only) }\end{array}$ & $\mid$\begin{tabular}{|l} 
Daylight \\
Input (if \\
applicable)
\end{tabular} & $\begin{array}{l}\text { Reference webpage } \\
\text { or other location } \\
\text { (see } \\
\text { "Reference.doc") }\end{array}$ & \begin{tabular}{|l|} 
Comment Description: \\
Issues, Suggestions \& \\
Requests
\end{tabular} & Action/Solution & \begin{tabular}{|l|} 
Status \\
or \\
Ticket \#
\end{tabular} & \begin{tabular}{|c|} 
Frequenc \\
$\mathrm{y}(20 \%)$
\end{tabular} & $\begin{array}{c}\text { Importance } \\
\text { of comment } \\
(80 \%)\end{array}$ & $\begin{array}{c}\text { Sum } \\
(100 \%)\end{array}$ & $\begin{array}{c}\text { Ease of } \\
\text { Implementatio } \\
\mathrm{n}(30 \%)\end{array}$ & $\begin{array}{c}\text { Duration } \\
(40 \%)\end{array}$ & $\begin{array}{c}\text { Risk/ } \\
\text { Complicatio } \\
\mathrm{n}(30 \%)\end{array}$ & \begin{tabular}{|} 
Sum \\
$(100 \%)$
\end{tabular} & \begin{tabular}{|c|} 
Short Term \\
$(40 \%$ \\
Importance) \\
$(60 \%$ Feasibility) \\
\end{tabular} & $\begin{array}{c}\text { Long Term } \\
(85 \% \\
\text { Importance) } \\
\text { (15\% Feasibility) }\end{array}$ \\
\hline M Myer & MM52 & Internal & Content & \begin{tabular}{|l|} 
Project \\
Description
\end{tabular} & & & & Operating hours & $\begin{array}{l}\text { I think that you can remove } \\
\text { the } 24 \text { hour button for an } \\
\text { office building }\end{array}$ & UI & 49 & 2 & 4 & 3.6 & 3 & 4 & 4 & 3.7 & 3.6 & 3.6 \\
\hline M Myer & MM51 & Internal & Functionality & \begin{tabular}{|l|} 
Project \\
Description
\end{tabular} & & & & Operating hours & \begin{tabular}{|l|} 
Recommend haivng a \\
weekday/weekend feature. \\
Retail changes look great. \\
Why not allow a cleaning \\
time for the operating \\
hours. You might also have \\
a percentage of staff during \\
off-period hours. For \\
example, a $100 \%$ of the \\
staff is expected between \\
$8: 00$ am and $6: 00$ pm. $50 \%$ \\
of the staff is there until \\
$8: 00 \mathrm{pm}$.
\end{tabular} & \begin{tabular}{|l} 
UI \\
\end{tabular} & 49 & 2 & 4 & 3.6 & 3 & 4 & 4 & 3.7 & 3.6 & 3.6 \\
\hline K Cota & KC1 & Utility & & & & & & $\begin{array}{l}\text { Kelly Cota, National } \\
\text { Grid }\end{array}$ & \begin{tabular}{|l|} 
what if you don't have your \\
furniture plan layout, can \\
you answer with a "0" or \\
N/A?
\end{tabular} & UI & 50 & 2 & 4 & 3.6 & 4 & 4 & 4 & 4 & 3.8 & 3.7 \\
\hline W Dau & WD3 & $\begin{array}{l}\text { Designer/IES } \\
\text { Chair }\end{array}$ & & & & & & & $\begin{array}{l}\text { I don't know what the size } \\
\text { of the window openings } \\
\text { are. }\end{array}$ & UI & 50 & 2 & 4 & 3.6 & 4 & 4 & 4 & 4 & 3.8 & 3.7 \\
\hline HMcKay & HM38.2 & Designer & & & & & & & \begin{tabular}{|l|} 
\\
Controls tab is missing the \\
icons and \\
explanations.
\end{tabular} & UI & 56 & 2 & 4 & 3.6 & 4 & 4 & 4 & 4 & 3.8 & 3.7 \\
\hline
\end{tabular}


Appendix E: Master Comments List

\begin{tabular}{|c|c|c|c|c|c|c|c|c|c|c|c|c|c|c|c|c|c|c|c|c|}
\hline $\begin{array}{l}\text { Commenter } \\
\text { ID \# }\end{array}$ & \begin{tabular}{|l|} 
Comment \\
Reference \\
$\#$
\end{tabular} & $\begin{array}{l}\text { Commenter } \\
\text { Category }\end{array}$ & $\begin{array}{l}\text { Comment } \\
\text { Type }\end{array}$ & Space Type & \begin{tabular}{|l|} 
Ceiling \\
Height/ \\
Total Area
\end{tabular} & \begin{tabular}{|l|}
$\#$ \\
workstations/ \\
partition \\
height (open \\
office only) \\
\end{tabular} & $\mid \begin{array}{l}\text { Daylight } \\
\text { Input (if } \\
\text { applicable) }\end{array}$ & \begin{tabular}{|l|} 
Reference webpage \\
or other location \\
(see \\
"Reference.doc") \\
\end{tabular} & $\begin{array}{l}\text { Comment Description: } \\
\text { Issues, Suggestions \& } \\
\text { Requests }\end{array}$ & Action/Solution & \begin{tabular}{|l|} 
Status \\
or \\
Ticket \#
\end{tabular} & $\begin{array}{c}\text { Frequenc } \\
\text { y (20\%) }\end{array}$ & $\begin{array}{c}\text { Importance } \\
\text { of comment } \\
(80 \%)\end{array}$ & \begin{tabular}{|c|} 
Sum \\
$(100 \%)$
\end{tabular} & $\begin{array}{c}\text { Ease of } \\
\text { Implementatio } \\
\mathrm{n}(30 \%)\end{array}$ & \begin{tabular}{|c} 
Duration \\
$(40 \%)$
\end{tabular} & $\begin{array}{c}\text { Risk/ } \\
\text { Complicatio } \\
\mathrm{n}(30 \%)\end{array}$ & \begin{tabular}{|} 
Sum \\
$(100 \%)$
\end{tabular} & \begin{tabular}{|c|} 
Short Term \\
$(40 \%$ \\
Importance) \\
(60\% Feasibility)
\end{tabular} & $\begin{array}{c}\text { Long Term } \\
\text { (85\% } \\
\text { Importance) } \\
\text { (15\% Feasibility) }\end{array}$ \\
\hline C DiLouie & CD2 & Core Team & $\begin{array}{l}\text { Ease of } \\
\text { Use/Flow }\end{array}$ & & & & & & $\begin{array}{l}\text { On the main page, it says } \\
\text { "select building type," but } \\
\text { the right box asks for } \\
\text { operating hours related to } \\
\text { stock and restocking, } \\
\text { implying office is not } \\
\text { included. That threw me off. } \\
\text { I suggest not having the } \\
\text { operating hours box } \\
\text { there or making it generic. I } \\
\text { also suggest asking the } \\
\text { user what building } \\
\text { type and make the choices } \\
\text { "retail" and "office," then } \\
\text { give those users } \\
\text { choosing retail the ability to } \\
\text { select a type of retail } \\
\text { building. }\end{array}$ & UI & 49 & 3 & 4 & 3.8 & 4 & 4 & 5 & 4.3 & 4.0 & 3.9 \\
\hline HMcKay & HM1 & Designer & \begin{tabular}{|l|} 
Ease of \\
Use/Flow
\end{tabular} & & & & & & $\begin{array}{l}\text { Needs auto-save or regular } \\
\text { prompts to save. Easy to } \\
\text { lose hours of input and } \\
\text { discourage use. } \\
\end{array}$ & UI & 53 & 3 & 4 & 3.8 & 4 & 4 & 5 & 4.3 & 4.0 & 3.9 \\
\hline W Dau & WD9 & \begin{tabular}{|l|} 
Designer/IES \\
Chair
\end{tabular} & & & & & & & $\begin{array}{l}\text { When I came back to the } \\
\text { project after having logged } \\
\text { out, there was no option for } \\
\text { opening an existing project } \\
\text { Perhaps I didn't save? the } \\
\text { system should ask before } \\
\text { letting me exit. } \\
\end{array}$ & UI & 53 & 3 & 4 & 3.8 & 4 & 4 & 5 & 4.3 & 4.0 & 3.9 \\
\hline J Yorgey & JY14 & Manufacturer & Functionality & & & & & & $\begin{array}{l}\text { Private office daylight detail } \\
\text { questions - not clear if per } \\
\text { room or total }\end{array}$ & UI & 46 & 1 & 4 & 3.4 & 5 & 5 & 5 & 5 & 4.0 & 3.6 \\
\hline A Cortese & AC10 & Architect & $\begin{array}{l}\text { Ease of } \\
\text { Use/Flow }\end{array}$ & & & & & & \begin{tabular}{|l|} 
Under the Luminaire tab I \\
wonder if the lighting \\
characteristics would read \\
easier if the attributes were \\
a bulleted list as opposed \\
to written in paragraph \\
form.
\end{tabular} & UI & 52 & 2 & 4 & 3.6 & 5 & 5 & 4 & 4.7 & 4.0 & 3.8 \\
\hline \begin{tabular}{|l} 
D Maniccia \\
\end{tabular} & DM10 & Controls & & & & & & Controls tab & \begin{tabular}{|l|} 
The vacancy sensor icon \\
could be improved. \\
(something that's not a light \\
bulb??)
\end{tabular} & UI & 51 & 3 & 4 & 3.8 & 4 & 5 & 4 & 4.4 & 4.0 & 3.9 \\
\hline HMcKay & HM23 & Designer & \begin{tabular}{|l|} 
Appearance| \\
Aesthetics
\end{tabular} & & & & & & \begin{tabular}{|l|} 
Icon for multilevel switching \\
is incorrect. It shows three \\
slide dimmers, not three \\
switches. Confusing.
\end{tabular} & UI & 51 & 3 & 4 & 3.8 & 4 & 5 & 4 & 4.4 & 4.0 & 3.9 \\
\hline M Myer & \begin{tabular}{|l|} 
MM55 \\
\end{tabular} & Internal & \begin{tabular}{|l|} 
Appearance| \\
Aesthetics
\end{tabular} & Private Office & & & & \begin{tabular}{|l|} 
Office Design \\
Vignettes
\end{tabular} & \begin{tabular}{|l} 
Brief case is graphic \\
effiment. While I am pro- \\
gender equality can we \\
have a gender neutral \\
graphic?
\end{tabular} & UI & 51 & 3 & 4 & 3.8 & 4 & 5 & 4 & 4.4 & 4.0 & 3.9 \\
\hline M Myer & \begin{tabular}{|l|} 
MM53 \\
\end{tabular} & Internal & \begin{tabular}{|l|} 
Appearancel \\
Aesthetics
\end{tabular} & Open Office & & & & \begin{tabular}{|l|} 
Office Design \\
Vignettes
\end{tabular} & $\begin{array}{l}\text { Can we explore a different } \\
\text { graphic, I do not think that } \\
\text { this is intuitive }\end{array}$ & UI & 51 & 3 & 4 & 3.8 & 4 & 5 & 4 & 4.4 & 4.0 & 3.9 \\
\hline
\end{tabular}




\begin{tabular}{|c|c|c|c|c|c|c|c|c|c|c|c|c|c|c|c|c|c|c|c|c|}
\hline $\begin{array}{l}\text { Commenter } \\
\text { ID \# }\end{array}$ & \begin{tabular}{|l|} 
Comment \\
Reference \\
$\#$
\end{tabular} & $\begin{array}{l}\text { Commenter } \\
\text { Category }\end{array}$ & $\begin{array}{l}\text { Comment } \\
\text { Type }\end{array}$ & Space Type & \begin{tabular}{|l|} 
Ceiling \\
Height/ \\
Total Area
\end{tabular} & \begin{tabular}{|l|}
$\#$ \\
workstations/ \\
partition \\
height (open \\
office only) \\
\end{tabular} & $\left|\begin{array}{l}\text { Daylight } \\
\text { Input (if } \\
\text { applicable) }\end{array}\right|$ & \begin{tabular}{|l|} 
Reference webpage \\
or other location \\
(see \\
"Reference.doc")
\end{tabular} & $\begin{array}{l}\text { Comment Description: } \\
\text { Issues, Suggestions \& } \\
\text { Requests }\end{array}$ & Action/Solution $/$ S & $\begin{array}{l}\text { Status } \\
\text { or } \\
\text { Ticket \# }\end{array}$ & $\begin{array}{c}\text { Frequenc } \\
\mathrm{y}(20 \%)\end{array}$ & $\begin{array}{c}\text { Importance } \\
\text { of comment } \\
(80 \%)\end{array}$ & $\begin{array}{c}\text { Sum } \\
(100 \%)\end{array}$ & $\begin{array}{c}\text { Ease of } \\
\text { Implementatio } \\
\mathrm{n}(30 \%)\end{array}$ & $\begin{array}{c}\text { Duration } \\
(40 \%)\end{array}$ & $\begin{array}{c}\text { Risk/ } \\
\text { Complicatio } \\
\mathrm{n}(30 \%)\end{array}$ & $\begin{array}{c}\text { Sum } \\
(100 \%)\end{array}$ & $\begin{array}{c}\text { Short Term } \\
(40 \% \\
\text { Importance) } \\
(60 \% \text { Feasibility) }\end{array}$ & $\begin{array}{c}\text { Long Term } \\
(85 \% \\
\text { Importance) } \\
(15 \% \text { Feasibility) }\end{array}$ \\
\hline M Myer & MM54 & $\mid$ Internal & \begin{tabular}{l|} 
Appearancet \\
Aesthetics
\end{tabular} & Reception & & & & \begin{tabular}{|l} 
Vffice Design \\
Vignettes
\end{tabular} & $\begin{array}{l}\text { I am not a fan of the happy } \\
\text { face. }\end{array}$ & $\overline{\mathrm{UI}}$ & 51 & 3 & 4 & 3.8 & 4 & 5 & 4 & 4.4 & 4.0 & 3.9 \\
\hline N Miller & \begin{tabular}{|l|} 
NM7 \\
\end{tabular} & Internal & General & \begin{tabular}{|l|} 
Pharmacy Des \\
Vignettes
\end{tabular} & & & & Icon for "perimeter" & \begin{tabular}{|l|} 
Sorry. I first interpreted the \\
icon as a shoebox \\
luminaire and pole seen in \\
plan. I didn't realize it was \\
one of the space options.
\end{tabular} & UI & 51 & 3 & 4 & 3.8 & 4 & 5 & 4 & 4.4 & 4.0 & 3.9 \\
\hline \begin{tabular}{|l|} 
C Dilouie \\
\end{tabular} & $\mathrm{CD7}$ & Core Team & \begin{tabular}{|l|} 
Ease of \\
Use/Flow
\end{tabular} & & & & & & $\begin{array}{l}\text { I clicked private office, } \\
\text { inputted space } \\
\text { characteristics, then } \\
\text { second box for daylighting } \\
\text { came up, but I had to scroll: } \\
\text { I shouldn't have to scroll? }\end{array}$ & UI & 54 & 5 & 4 & 4.2 & 4 & 4 & 4 & 4 & 4.1 & 4.2 \\
\hline D Maniccia & DM5 & Controls & & & & & & & \begin{tabular}{|l|} 
When you hit next \\
(because you can't see the \\
menu for VT until you \\
scroll) it doesn't let you go \\
forward but I can't see why. \\
Can you enlarge the box?
\end{tabular} & UI & 54 & 5 & 4 & 4.2 & 4 & 4 & 4 & 4 & 4.1 & 4.2 \\
\hline HMcKay & HM7 & Designer & \begin{tabular}{|c|} 
Functionality \\
\end{tabular} & & & & & & \begin{tabular}{|l|} 
Window size for Add an \\
Open Plan space is too \\
short. Completely missed \\
daylight availability \\
question. Box for windows \\
should have \\
yes or no option. \\
Otherwise, if left \\
unchecked, no windows \\
appear, but the user \\
doesn't know there was a \\
choice.
\end{tabular} & UI & 54 & 5 & 4 & 4.2 & 4 & 4 & 4 & 4 & 4.1 & 4.2 \\
\hline HMcKay & HM6 & Designer & \begin{tabular}{|l|} 
Functionality \\
\end{tabular} & & & & VT & & \begin{tabular}{|l|} 
Window size for vignette \\
options seems too short; \\
user may think only 2 \\
options. Maybe indicate \\
how many vignettes are \\
available, or \\
increase size of window to \\
show top of third vignette. \\
\end{tabular} & UI & 54 & 5 & 4 & 4.2 & 4 & 4 & 4 & 4 & 4.1 & 4.2 \\
\hline \begin{tabular}{|l|l|} 
LDavis \\
\end{tabular} & LD6 & Designer & & & & & & & \begin{tabular}{|l|} 
I didn't know that there was \\
a checkmark for windows \\
because I didn't scroll \\
down, you shouldn't be \\
able to hit "next" and miss \\
the chance to put in \\
window information. Can't \\
you make the box bigger, \\
especially since there is \\
only one more box in the \\
list?
\end{tabular} & UI & 54 & 5 & 4 & 4.2 & 4 & 4 & 4 & 4 & 4.1 & 4.2 \\
\hline M Dare & MD4 & Utility & & & & & & & $\begin{array}{l}\text { Missed the windows } \\
\text { checkbox, you shouldn't let } \\
\text { people go forward without } \\
\text { knowing that box is there. }\end{array}$ & UI & 54 & 5 & 4 & 4.2 & 4 & 4 & 4 & 4 & 4.1 & 4.2 \\
\hline
\end{tabular}




\begin{tabular}{|c|c|c|c|c|c|c|c|c|c|c|c|c|c|c|c|c|c|c|c|c|}
\hline $\begin{array}{l}\text { Commenter } \\
\text { ID \# }\end{array}$ & \begin{tabular}{|l|} 
Comment \\
Reference \\
$\#$
\end{tabular} & $\begin{array}{l}\text { Commenter } \\
\text { Category }\end{array}$ & \begin{tabular}{|l} 
Comment \\
Type
\end{tabular} & Space Type & \begin{tabular}{|l} 
Ceiling \\
Height/ \\
Total Area
\end{tabular} & \begin{tabular}{|l}
$\#$ \\
workstations/ \\
partition \\
height (open \\
office only) \\
\end{tabular} & \begin{tabular}{|l|} 
Daylight \\
Input (if \\
applicable)
\end{tabular} & \begin{tabular}{|l|} 
Reference webpage \\
or other location \\
(see \\
"Reference.doc") \\
\end{tabular} & \begin{tabular}{l|} 
Comment Description: \\
Issues, Suggestions \& \\
Requests
\end{tabular} & \begin{tabular}{|l|l} 
Action/Solution & \\
&
\end{tabular} & \begin{tabular}{|l|}
$\begin{array}{l}\mid l \\
\text { Status } \\
\text { or } \\
\text { Ticket \# }\end{array}$ \\
\end{tabular} & $\begin{array}{c}\text { Frequenc } \\
\text { y (20\%) }\end{array}$ & $\begin{array}{c}\text { Importance } \\
\text { of comment } \\
(80 \%)\end{array}$ & \begin{tabular}{|l|} 
Sum \\
$(100 \%)$
\end{tabular} & $\begin{array}{c}\text { Ease of } \\
\text { Implementatio } \\
\mathrm{n}(30 \%)\end{array}$ & $\begin{array}{c}\text { Duration } \\
(40 \%)\end{array}$ & $\begin{array}{c}\text { Risk/ } \\
\text { Complicatio } \\
\mathrm{n}(30 \%)\end{array}$ & \begin{tabular}{|c|} 
Sum \\
$(100 \%)$
\end{tabular} & $\begin{array}{c}\text { Short Term } \\
(40 \% \\
\text { Importance) } \\
(60 \% \text { Feasibility) } \\
\end{array}$ & $\begin{array}{c}\text { Long Term } \\
(85 \% \\
\text { Importance) } \\
\text { (15\% Feasibility) } \\
\end{array}$ \\
\hline A Cortese & AC25 & Architect & Functionality & & & & & & $\begin{array}{l}\text { When I first started using } \\
\text { the system, I was very } \\
\text { interested in seeing the } \\
\text { guided tour, but I never } \\
\text { could get that to work. }\end{array}$ & $\overline{\mathrm{UI}}$ & 44 & 2 & 4 & 3.6 & 5 & 5 & 5 & 5 & 4.2 & 3.8 \\
\hline N Miller & NM6 & Internal & Functionality & CLS intro page & & & & Guided Tour & $\begin{array}{l}\text { The Guided Tour took me } \\
\text { to a mini sign-in page and } \\
\text { no further. It must not be } \\
\text { ready yet. }\end{array}$ & UI & 44 & 2 & 4 & 3.6 & 5 & 5 & 5 & 5 & 4.2 & 3.8 \\
\hline C DiLouie & CD14 & Core Team & Functionality & & & & & & $\begin{array}{l}\text { After finishing, the edit and } \\
\text { delete icons only come up } \\
\text { when I scroll over them - } \\
\text { they should come up } \\
\text { automatically }\end{array}$ & UI & 55 & 3 & 5 & 4.6 & 4 & 4 & 4 & 4 & 4.4 & 4.5 \\
\hline HMcKay & HM17 & Designer & Content & & & & & & $\begin{array}{l}\text { Summary of space } \\
\text { description lists partition } \\
\text { height as ft, not inches. }\end{array}$ & UI & 55 & 3 & 5 & 4.6 & 4 & 4 & 4 & 4 & 4.4 & 4.5 \\
\hline J Yorgey & JY29 & Manufacturer & Functionality & & & & & $\begin{array}{l}\text { Office design } \\
\text { vinettes }\end{array}$ & $\begin{array}{l}\text { "corridor spaces" a edit } \\
\text { /delete symbols come up } \\
\text { but only when cursor is } \\
\text { over listed space. These } \\
\text { sybols do not appear on } \\
\text { other space type pages }\end{array}$ & UI & 55 & 3 & 5 & 4.6 & 4 & 4 & 4 & 4 & 4.4 & 4.5 \\
\hline M Myer & MM634 & Internal & Content & \begin{tabular}{|l|} 
Controls \\
Strategies: \\
Scheduling, \\
Daylight \\
Harvesting \\
\end{tabular} & & & & Images & $\begin{array}{l}\text { This is the first time that we } \\
\text { have mouse-overs in the } \\
\text { tool. We should make it } \\
\text { obvious that these are } \\
\text { mouse overs }\end{array}$ & UI & 47 & 2 & 5 & 4.4 & 4 & 4 & 5 & 4.3 & 4.4 & 4.4 \\
\hline C DiLouie & CD12 & Core Team & \begin{tabular}{|l|} 
Ease of \\
Use/Flow
\end{tabular} & & & & & & \begin{tabular}{|l|} 
After finishing, it gives me \\
a button "new space," \\
which I clicked, and it \\
started me on a new \\
private office, but I had \\
thought "new space" meant \\
I would revisit the key \\
plan-maybe offer two \\
buttons there, or again, \\
make the path back to key \\
plan very legible \\
\end{tabular} & UI & 9 & 3 & 5 & 4.6 & 4 & 4 & 5 & 4.3 & 4.5 & 4.6 \\
\hline C Dilouie & CD10 & Core Team & $\mid$ Functionality & & & & & & \begin{tabular}{|l|} 
I should always have a very \\
legible, clear path back to \\
key plan; since the path link \\
is above where I'm working \\
and not below, where I \\
would expect it to be, it \\
should be very legible
\end{tabular} & UI & 9 & 3 & 5 & 4.6 & 4 & 4 & 5 & 4.3 & 4.5 & 4.6 \\
\hline
\end{tabular}


Appendix E: Master Comments List

\begin{tabular}{|c|c|c|c|c|c|c|c|c|c|c|c|c|c|c|c|c|c|c|c|}
\hline $\begin{array}{l}\text { Commenter } \\
\text { ID \# }\end{array}$ & $\begin{array}{l}\text { Comment } \\
\text { Reference } \\
\#\end{array}$ & $\begin{array}{l}\text { Commenter } \\
\text { Category }\end{array}$ & \begin{tabular}{|l} 
Comment \\
Type
\end{tabular} & Space Type & \begin{tabular}{|l|} 
Ceiling \\
Height/ \\
Total Area
\end{tabular} & \begin{tabular}{|l|}
$\#$ \\
workstations/ \\
partition \\
height (open \\
office only) \\
\end{tabular} & \begin{tabular}{|l|} 
Daylight \\
Input (if \\
applicable)
\end{tabular} & $\begin{array}{l}\text { Reference webpage } \\
\text { or other location } \\
\text { (see } \\
\text { "Reference.doc") } \\
\end{array}$ & $\begin{array}{l}\text { Comment Description: } \\
\text { Issues, Suggestions \& } \\
\text { Requests }\end{array}$ & 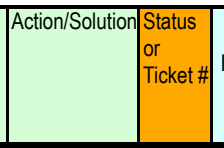 & $\begin{array}{l}\text { Frequenc } \\
\text { y (20\%) }\end{array}$ & $\begin{array}{c}\text { Importance } \\
\text { of comment } \\
(80 \%)\end{array}$ & \begin{tabular}{|c|} 
Sum \\
$(100 \%)$
\end{tabular} & $\begin{array}{c}\text { Ease of } \\
\text { Implementatio } \\
\mathrm{n}(30 \%)\end{array}$ & $\begin{array}{c}\text { Duration } \\
(40 \%)\end{array}$ & $\begin{array}{c}\text { Risk/ } \\
\text { Complicatio } \\
\mathrm{n}(30 \%)\end{array}$ & \begin{tabular}{|} 
Sum \\
$(100 \%)$
\end{tabular} & 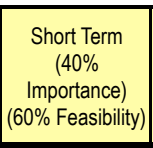 & 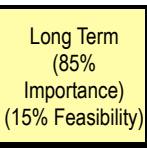 \\
\hline C Dilouie & CD5 & Core Team & \begin{tabular}{|l|} 
Ease of \\
Use/Flow
\end{tabular} & & & & & & $\begin{array}{l}\text { Then, after asking for more } \\
\text { information about controls, } \\
\text { I found I couldn't } \\
\text { close that screen, and } \\
\text { subsequently I couldn't get } \\
\text { back to my decisionmaking } \\
\text { process until I closed the } \\
\text { window by clicking the } \\
\text { upper righthand corner } \\
\text { (meaning I had given up } \\
\text { and thought I had to start } \\
\text { over). In other words } \\
\text { there should be a button } \\
\text { that says cclose this } \\
\text { screen" on the controls } \\
\text { page }\end{array}$ & UI & 3 & 5 & 4.6 & 4 & 4 & 5 & 4.3 & 4.5 & 4.6 \\
\hline HMcKay & HM4 & Designer & \begin{tabular}{|l|} 
Ease of \\
Use/Flow
\end{tabular} & & & & & & $\begin{array}{l}\text { Should have exaggerated } \\
\text { CLOSE X to close vignette } \\
\text { and template detail pages } \\
\text { or a BACK button. }\end{array}$ & UI & 3 & 5 & 4.6 & 4 & 4 & 5 & 4.3 & 4.5 & 4.6 \\
\hline L Davis & LD5 & Designer & & & & & & & $\begin{array}{l}\text { I found the Firefox windows } \\
\text { confusing, the program } \\
\text { opens new windows and I } \\
\text { had trouble knowing where } \\
\text { the action was. }\end{array}$ & UI & 3 & 5 & 4.6 & 4 & 4 & 5 & 4.3 & 4.5 & 4.6 \\
\hline L Davis & LD9 & Designer & & & & & & & $\begin{array}{l}\text { When I'm done looking at } \\
\text { the vignette details, it's not } \\
\text { immediately apparent that } 1 \\
\text { should close the box, or } \\
\text { how to get back to the other } \\
\text { screen. Nervous to close } \\
\text { box, because it might shut } \\
\text { down the tool. When you } \\
\text { click on options, it takes } \\
\text { you back to primary } \\
\text { window, not clear to me } \\
\text { immediately. }\end{array}$ & UI & 3 & 5 & 4.6 & 4 & 4 & 5 & 4.3 & 4.5 & 4.6 \\
\hline M Myer & MM56 & Internal & \begin{tabular}{|l|} 
Functionality \\
\end{tabular} & Reception & & & & Reception Spaces & $\begin{array}{l}\text { "New space" button. Can } \\
\text { this blink when I have not } \\
\text { created any spaces? }\end{array}$ & UI & 3 & 5 & 4.6 & 4 & 4 & 5 & 4.3 & 4.5 & 4.6 \\
\hline
\end{tabular}




\begin{tabular}{|c|c|c|c|c|c|c|c|c|c|c|c|c|c|c|c|c|c|c|c|c|}
\hline $\begin{array}{l}\text { Commenter } \\
\text { ID\# \# }\end{array}$ & $\begin{array}{l}\text { Comment } \\
\text { Reference } \\
\#\end{array}$ & $\begin{array}{l}\text { Commenter } \\
\text { Category }\end{array}$ & $\begin{array}{l}\text { Comment } \\
\text { Type }\end{array}$ & Space Type & \begin{tabular}{|l|} 
Ceiling \\
Height/ \\
Total Area
\end{tabular} & \begin{tabular}{|l|}
$\#$ \\
workstations/ \\
partition \\
height (open \\
office only) \\
\end{tabular} & $\mid$\begin{tabular}{|l|} 
Daylight \\
Input (if \\
applicable)
\end{tabular} & 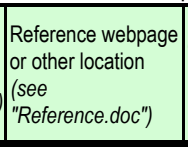 & \begin{tabular}{|l|} 
Comment Description: \\
Issues, Suggestions \& \\
Requests
\end{tabular} & Action/Solution & \begin{tabular}{|l|} 
Status \\
or \\
Ticket \#
\end{tabular} & $\begin{array}{c}\text { Frequenc } \\
\text { y (20\%) }\end{array}$ & $\begin{array}{c}\text { Importance } \\
\text { of comment } \\
(80 \%)\end{array}$ & $\begin{array}{c}\text { Sum } \\
(100 \%)\end{array}$ & $\begin{array}{c}\text { Ease of } \\
\text { Implementatio } \\
\mathrm{n}(30 \%)\end{array}$ & \begin{tabular}{|c|} 
Duration \\
$(40 \%)$
\end{tabular} & $\begin{array}{c}\text { Risk/ } \\
\text { Complicatio } \\
\mathrm{n}(30 \%)\end{array}$ & \begin{tabular}{|} 
Sum \\
$(100 \%)$
\end{tabular} \mid & 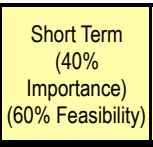 & $\begin{array}{c}\text { Long Term } \\
(85 \% \\
\text { Importance) } \\
\text { (15\% Feasibility) } \\
\end{array}$ \\
\hline N Ferzacca & NF10 & Engineer & & & & & & & \begin{tabular}{|l|} 
How do I close this? \\
(vignette window) Took a \\
minute to realize how to get \\
back to the other window.
\end{tabular} & UI & 9 & 3 & 5 & 4.6 & 4 & 4 & 5 & 4.3 & 4.5 & 4.6 \\
\hline N Ferzacca & NF16 & Engineer & & & & & & & \begin{tabular}{|l|} 
The next button did not \\
show up in the size of \\
window at first, had to \\
make it bigger, needed \\
input to know to enlarge the \\
window.
\end{tabular} & UI & 9 & 3 & 5 & 4.6 & 4 & 4 & 5 & 4.3 & 4.5 & 4.6 \\
\hline N Ferzacca & NF14 & Engineer & & & & & & & \begin{tabular}{|l|} 
Wasn't obvious to me that \\
the key plan button was the \\
next step. Perhaps add a \\
next button as well as the \\
key plan button? Or make \\
it more obvious?
\end{tabular} & UI & 9 & 3 & 5 & 4.6 & 4 & 4 & 5 & 4.3 & 4.5 & 4.6 \\
\hline N Miller & NM13 & Internal & & & & & & & $\begin{array}{l}\text { No way to navigate back to } \\
\text { previous page. }\end{array}$ & UI & 9 & 3 & 5 & 4.6 & 4 & 4 & 5 & 4.3 & 4.5 & 4.6 \\
\hline N Miller & NM21 & Internal & & & & & & & \begin{tabular}{|l|} 
\\
How do you navigate back \\
to the model you're \\
building? Maybe that \\
doesn't work yet.
\end{tabular} & UI & 9 & 3 & 5 & 4.6 & 4 & 4 & 5 & 4.3 & 4.5 & 4.6 \\
\hline N Miller & NM5.1 & Internal & Content & $\begin{array}{l}\text { Pharm - Genl } \\
\text { Merch }\end{array}$ & & & & & $\begin{array}{l}\text { When I was done with this } \\
\text { page, I couldn't figure out } \\
\text { where to go. So I hit the } \\
\text { back button. Ooops. I } \\
\text { must have missed } \\
\text { something. }\end{array}$ & UI & 9 & 3 & 5 & 4.6 & 4 & 4 & 5 & 4.3 & 4.5 & 4.6 \\
\hline
\end{tabular}




\begin{tabular}{|c|c|c|c|c|c|c|c|c|c|c|c|c|c|c|c|c|c|c|c|c|}
\hline $\begin{array}{l}\text { Commenter } \\
\text { ID \# }\end{array}$ & \begin{tabular}{|l|} 
Comment \\
Reference \\
$\#$
\end{tabular} & $\begin{array}{l}\text { Commenter } \\
\text { Category }\end{array}$ & \begin{tabular}{|l} 
Comment \\
Type
\end{tabular} & Space Type & \begin{tabular}{|l|} 
Ceiling \\
Height/ \\
Total Area
\end{tabular} & \begin{tabular}{|l|}
$\#$ \\
workstations/ \\
partition \\
height (open \\
office only) \\
\end{tabular} & $\left|\begin{array}{l}\text { Daylight } \\
\text { Input (if } \\
\text { applicable) }\end{array}\right|$ & \begin{tabular}{|l|} 
Reference webpage \\
or other location \\
(see \\
"Reference.doc")
\end{tabular} & $\begin{array}{l}\text { Comment Description: } \\
\text { Issues, Suggestions \& } \\
\text { Requests }\end{array}$ & Action/Solution/s: & \begin{tabular}{|l|}
$\begin{array}{l}\text { Status } \\
\text { or } \\
\text { Ticket \# }\end{array}$ \\
\end{tabular} & $\begin{array}{c}\text { Frequenc } \\
\mathrm{y}(20 \%)\end{array}$ & $\begin{array}{c}\text { Importance } \\
\text { of comment } \\
(80 \%)\end{array}$ & \begin{tabular}{|} 
Sum \\
$(100 \%)$
\end{tabular} & $\begin{array}{c}\text { Ease of } \\
\text { Implementatio } \\
\mathrm{n}(30 \%)\end{array}$ & \begin{tabular}{|c|} 
Duration \\
$(40 \%)$
\end{tabular} & $\begin{array}{c}\text { Risk/ } \\
\text { Complicatio } \\
\mathrm{n}(30 \%)\end{array}$ & \begin{tabular}{|} 
Sum \\
$(100 \%)$
\end{tabular} \mid & \begin{tabular}{|c|} 
Short Term \\
$(40 \%$ \\
Importance) \\
$(60 \%$ Feasibility) \\
\end{tabular} & $\begin{array}{c}\text { Long Term } \\
(85 \% \\
\text { Importance) } \\
\text { (15\% Feasibility) }\end{array}$ \\
\hline W Dau & WD10 & $\begin{array}{l}\begin{array}{l}\text { Designer/IES } \\
\text { Chair }\end{array} \\
\end{array}$ & & & & & & & $\begin{array}{l}\text { The 'back to the key plan } \\
\text { button/link should be } \\
\text { brighter, the off color } \\
\text { doesn't give the impression } \\
\text { of it being a button }\end{array}$ & $\overline{\mathrm{UI}}$ & 9 & 3 & 5 & 4.6 & 4 & 4 & 5 & 4.3 & 4.5 & 4.6 \\
\hline W Dau & WD4 & \begin{tabular}{|l|}
$\begin{array}{l}\text { Designer/IES } \\
\text { Chair }\end{array}$ \\
\end{tabular} & & & & & & & \begin{tabular}{|l|l} 
I went right past the \\
vignette details. That little \\
hyperlink is very often used \\
as a help file, doesn't read \\
as something that is \\
mission-critical for the \\
workflow process? \\
\end{tabular} & UI & 9 & 1 & 5 & 4.2 & 5 & 5 & 5 & 5 & 4.5 & 4.3 \\
\hline D Maniccia & DM4 & Controls & & & & & & & $\begin{array}{l}\text { Change the language to } \\
\text { read: How many rooms of } \\
\text { this type do you have? } \\
\text { Use of the word "space" is } \\
\text { problematic. }\end{array}$ & UI & 46 & 4 & 5 & 4.8 & 4 & 4 & 5 & 4.3 & 4.6 & 4.7 \\
\hline HMcKay & HM11 & Designer & Content & & & & & & \begin{tabular}{|l} 
How many glazed walls "to \\
outside" - (to distinguish \\
from interior glass walls).
\end{tabular} & UI & 46 & 4 & 5 & 4.8 & 4 & 4 & 5 & 4.3 & 4.6 & 4.7 \\
\hline M Dare & MD6 & Utility & & & & & & & $\begin{array}{l}\text { It took me a while to notice } \\
\text { the difference between } \\
\text { LENGTH of daylighting } \\
\text { wall" and "sidelighting } \\
\text { glazing AREA." }\end{array}$ & UI & 46 & 4 & 5 & 4.8 & 4 & 4 & 5 & 4.3 & 4.6 & 4.7 \\
\hline N Ferzacca & NF3 & Engineer & & & & & & & $\begin{array}{l}\text { You are asking for the } \\
\text { length of the daylighting } \\
\text { walls-- are you asking for } \\
\text { length of the windows? Or } \\
\text { the window walls? If you } \\
\text { are getting glazing area, } \\
\text { why do you need the length } \\
\text { of the daylighting walls? }\end{array}$ & UI & 46 & 4 & 5 & 4.8 & 4 & 4 & 5 & 4.3 & 4.6 & 4.7 \\
\hline P Lantine & PL1 & Utilities & & & & & & \begin{tabular}{|l} 
Paul Lentine, \\
Northeast Utilities
\end{tabular} & $\begin{array}{l}\text { need a help screen for } \\
\text { wizards }\end{array}$ & UI & 46 & 4 & 5 & 4.8 & 4 & 4 & 5 & 4.3 & 4.6 & 4.7 \\
\hline A Cortese & AC4 & Architect & General & & & & & & \begin{tabular}{|l|} 
wonder if by burying the \\
baseline code in the corner, \\
people are missing this and \\
defaulting to ASHRAE $90.1-$ \\
2007. Is there any way you \\
could elevate the code \\
question to the first input \\
screen?
\end{tabular} & UI & 43 & 5 & 5 & 5 & 4 & 4 & 5 & 4.3 & 4.7 & 4.9 \\
\hline
\end{tabular}




\begin{tabular}{|c|c|c|c|c|c|c|c|c|c|c|c|c|c|c|c|c|c|c|c|c|}
\hline $\begin{array}{l}\text { Commenter } \\
\text { ID \# }\end{array}$ & \begin{tabular}{|l|} 
Comment \\
Reference \\
$\#$
\end{tabular} & $\begin{array}{l}\text { Commenter } \\
\text { Category }\end{array}$ & $\begin{array}{l}\text { Comment } \\
\text { Type }\end{array}$ & Space Type & \begin{tabular}{|l|} 
Ceiling \\
Height/ \\
Total Area
\end{tabular} & \begin{tabular}{|l}
$\#$ \\
workstations/ \\
partition \\
height (open \\
office only) \\
\end{tabular} & $\mid \begin{array}{l}\text { Daylight } \\
\text { Input (if } \\
\text { applicable) }\end{array}$ & \begin{tabular}{|l|} 
Reference webpage \\
or other location \\
(see \\
"Reference.doc")
\end{tabular} & $\begin{array}{l}\text { Comment Description: } \\
\text { Issues, Suggestions \& } \\
\text { Requests }\end{array}$ & Action/Solution/ & \begin{tabular}{|l|}
$\begin{array}{l}\text { Status } \\
\text { or } \\
\text { Ticket \# }\end{array}$ \\
\end{tabular} & $\begin{array}{c}\text { Frequenc } \\
\mathrm{y}(20 \%)\end{array}$ & $\begin{array}{c}\text { Importance } \\
\text { of comment } \\
(80 \%)\end{array}$ & \begin{tabular}{|c|} 
Sum \\
$(100 \%)$
\end{tabular} & $\begin{array}{c}\text { Ease of } \\
\text { Implementatio } \\
\mathrm{n}(30 \%)\end{array}$ & $\begin{array}{c}\text { Duration } \\
(40 \%)\end{array}$ & $\begin{array}{c}\text { Risk/ } \\
\text { Complicatio } \\
\mathrm{n}(30 \%)\end{array}$ & \begin{tabular}{|} 
Sum \\
$(100 \%)$
\end{tabular} \mid & \begin{tabular}{|c|} 
Short Term \\
$(40 \%$ \\
Importance) \\
$(60 \%$ Feasibility) \\
\end{tabular} & $\begin{array}{c}\text { Long Term } \\
(85 \% \\
\text { Importance) } \\
\text { (15\% Feasibility) }\end{array}$ \\
\hline C Magee & CM8 & Owner & & & & & & & Missed the code selection. & $\mathrm{UI}$ & 43 & 5 & 5 & 5 & 4 & 4 & 5 & 4.3 & 4.7 & 4.9 \\
\hline D Maniccia & DM1 & Controls & & & & & & & \begin{tabular}{|l|} 
Why doesn't the energy \\
code baseline pre-populate \\
based on the state that \\
gets selected?
\end{tabular} & UI & 43 & 5 & 5 & 5 & 4 & 4 & 5 & 4.3 & 4.7 & 4.9 \\
\hline D Maniccia & DM2 & Controls & & & & & & & $\begin{array}{l}\text { Not everyone will notice the } \\
\text { code selection- can you } \\
\text { flag it if someone doesn't } \\
\text { select, instead of having it } \\
\text { default to } 90.1-2007 \text { ? }\end{array}$ & UI & 43 & 5 & 5 & 5 & 4 & 4 & 5 & 4.3 & 4.7 & 4.9 \\
\hline HMcKay & HM2 & Designer & $\begin{array}{l}\text { Ease of } \\
\text { Use/Flow }\end{array}$ & & & & & & \begin{tabular}{|l|} 
Should direct user to select \\
appropriate code. Not \\
noticeable to user.
\end{tabular} & UI & 43 & 5 & 5 & 5 & 4 & 4 & 5 & 4.3 & 4.7 & 4.9 \\
\hline L Davis & LD3 & Designer & & & & & & & \begin{tabular}{|l|} 
The drop down code menu \\
isn't obvious enough, I \\
could miss it entirely.
\end{tabular} & UI & 43 & 5 & 5 & 5 & 4 & 4 & 5 & 4.3 & 4.7 & 4.9 \\
\hline M Dare & MD1 & Utility & & & & & & & Missed the code menu. & UI & 43 & 5 & 5 & 5 & 4 & 4 & 5 & 4.3 & 4.7 & 4.9 \\
\hline N Ferzacca & NF1 & Engineer & & & & & & & $\begin{array}{l}\text { Missed the code selection } \\
\text { menu. }\end{array}$ & UI & 43 & 5 & 5 & 5 & 4 & 4 & 5 & 4.3 & 4.7 & 4.9 \\
\hline W Dau & WD1 & \begin{tabular}{|l} 
Designer/IES \\
Chair
\end{tabular} & & & & & & & $\begin{array}{l}\text { Missed the code selection } \\
\text { box, not obvious enough. }\end{array}$ & UI & 43 & 5 & 5 & 5 & 4 & 4 & 5 & 4.3 & 4.7 & 4.9 \\
\hline M Myer & MM627 & Internal & Content & \begin{tabular}{|l|} 
Perimeter \\
Recessed \\
Linear \\
Fluorescent \\
\end{tabular} & & & & $\begin{array}{l}\text { Strategy/Maintenan } \\
\text { ce }\end{array}$ & \begin{tabular}{|l|} 
I do not remember the last \\
paragraph related to \\
cleaning in other vignettes \\
with lensed wall washers \\
\end{tabular} & VignetteText & 17 & 1 & 3 & 2.6 & 4 & 4 & 4 & 4 & 3.2 & 2.8 \\
\hline M Myer & MM151 & Internal & Content & \begin{tabular}{|l|} 
Recessed \\
Compact \\
Fluorescent \\
Downlights with \\
Dropped \\
Decorative \\
Diffuser and \\
Perimeter Wall- \\
Slot (office) \\
\end{tabular} & & & & Approach/Criteria & \begin{tabular}{|l|} 
Why limit CRI for \\
fluorescent and not CFLs? I \\
recommend going with 82 \\
for both
\end{tabular} & \begin{tabular}{|l} 
VignetteText \\
\end{tabular} & 17 & 1 & 4 & 3.4 & 4 & 4 & 4 & 4 & 3.6 & 3.5 \\
\hline M Myer & MM110 & Internal & Content & \begin{tabular}{|l|} 
Recessed Slot \\
Luminaires \\
Parallel to Desk \\
with \\
Contiunous \\
Linear Wall- \\
washers (office)
\end{tabular} & & & & Luminaires C & $\begin{array}{l}\text { CRI of } 85 \text { seems high, why } \\
\text { not } 80+\text { or even } 82 \text {. }\end{array}$ & \begin{tabular}{|l|} 
VignetteText \\
\end{tabular} & 17 & 1 & 4 & 3.4 & 4 & 4 & 4 & 4 & 3.6 & 3.5 \\
\hline M Myer & MM515 & Internal & Content & \begin{tabular}{|l|} 
Recessed Non- \\
Planar (LPD \\
0.64 W/sf)
\end{tabular} & $8 / 5000$ & $30 / 48$ & & Luminaires C & \begin{tabular}{|l|} 
Why is the CRI for the \\
LEDs null. Although CRI is \\
not entirely accurate, many \\
LED manufacturers are \\
reporting the value. In the \\
future we will probably use \\
a different metric, but I think \\
that we should keep a \\
value for the time
\end{tabular} & \begin{tabular}{|l|} 
VignetteText \\
\end{tabular} & 17 & 1 & 4 & 3.4 & 4 & 4 & 4 & 4 & 3.6 & 3.5 \\
\hline
\end{tabular}




\begin{tabular}{|c|c|c|c|c|c|c|c|c|c|c|c|c|c|c|c|c|c|c|c|c|}
\hline $\begin{array}{l}\text { Commenter } \\
\text { ID \# }\end{array}$ & $\begin{array}{l}\text { Comment } \\
\text { Reference } \\
\#\end{array}$ & $\begin{array}{l}\text { Commenter } \\
\text { Category }\end{array}$ & \begin{tabular}{|l} 
Comment \\
Type
\end{tabular} & Space Type & \begin{tabular}{|l|} 
Ceiling \\
Height/ \\
Total Area
\end{tabular} & \begin{tabular}{|l}
$\#$ \\
workstations/ \\
partition \\
height (open \\
office only) \\
\end{tabular} & $\mid \begin{array}{l}\text { Daylight } \\
\text { Input (if } \\
\text { applicable) }\end{array}$ & \begin{tabular}{|l|} 
Reference webpage \\
or other location \\
(see \\
"Reference.doc")
\end{tabular} & $\begin{array}{l}\text { Comment Description: } \\
\text { Issues, Suggestions \& } \\
\text { Requests }\end{array}$ & Action/Solution & \begin{tabular}{|l|}
$\begin{array}{l}\text { Status } \\
\text { or } \\
\text { Ticket \# }\end{array}$ \\
\end{tabular} & $\begin{array}{c}\text { Frequenc } \\
\mathrm{y}(20 \%)\end{array}$ & $\begin{array}{c}\text { Importance } \\
\text { of comment } \\
(80 \%)\end{array}$ & \begin{tabular}{|c|} 
Sum \\
$(100 \%)$
\end{tabular} & $\begin{array}{c}\text { Ease of } \\
\text { Implementatio } \\
\mathrm{n}(30 \%)\end{array}$ & \begin{tabular}{|c|} 
Duration \\
$(40 \%)$
\end{tabular} & $\begin{array}{c}\text { Risk/ } \\
\text { Complicatio } \\
\mathrm{n}(30 \%)\end{array}$ & \begin{tabular}{|} 
Sum \\
$(100 \%)$
\end{tabular} \mid & \begin{tabular}{|c|} 
Short Term \\
$(40 \%$ \\
Importance) \\
$(60 \%$ Feasibility) \\
\end{tabular} & $\begin{array}{c}\text { Long Term } \\
(85 \% \\
\text { Importance) } \\
\text { (15\% Feasibility) }\end{array}$ \\
\hline M Myer & MM121 & 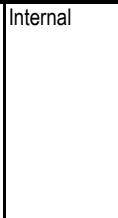 & Content & \begin{tabular}{l|} 
Recessed Slot \\
Luminaires \\
Parallel to Desk \\
with \\
Contiunous \\
Linear Wall- \\
washers (office)
\end{tabular} & & & & Approach/Criteria & $\begin{array}{l}\text { Why are we calling out an } \\
85 \text { or better CRI for linear } \\
\text { fluorescent and then } \\
\text { allowing } 82 \text { for CFLs. CRI } \\
\text { deviations of }+/-5 \text { are } \\
\text { impossible to tell. I say just } \\
\text { say } 82 \text { or better. }\end{array}$ & VignetteText & 17 & 1 & 4 & 3.4 & 4 & 4 & 4 & 4 & 3.6 & 3.5 \\
\hline C Dilouie & CD32 & Core Team & Content & & & & & & $\begin{array}{l}\text { Under "Long Term } \\
\text { Maintenance," there is no } \\
\text { text. }\end{array}$ & VignetteText & 17 & 2 & 4 & 3.6 & 4 & 5 & 5 & 4.7 & 4.0 & 3.8 \\
\hline K Abernathy & KA8 & Designer & General & Pharmacy & & & & \begin{tabular}{|l|} 
Vingettes for Center \\
aisle
\end{tabular} & $\begin{array}{l}\text { The same errors are } \\
\text { showing up in these } \\
\text { vingettes, symmetrical is } \\
\text { spelled wrong, }\end{array}$ & VignetteText & 17 & 2 & 4 & 3.6 & 4 & 5 & 5 & 4.7 & 4.0 & 3.8 \\
\hline K Abernathy & KA2 & Designer & General & Pharmacy & & & & \begin{tabular}{|l|} 
Luminaires Parallel \\
to Wall with \\
Skylights Pharmacy \\
under Luminaires
\end{tabular} & $\begin{array}{l}\text { The word symmetrical is } \\
\text { misspelled through out. }\end{array}$ & VignetteText & 17 & 2 & 4 & 3.6 & 4 & 5 & 5 & 4.7 & 4.0 & 3.8 \\
\hline K Abernathy & KA4 & Designer & General & Pharmacy & & & & \begin{tabular}{|l|} 
Luminaires Parallel \\
to Wall Pharmacy - \\
under Luminaires
\end{tabular} & $\begin{array}{l}\text { The word symmetrical is } \\
\text { misspelled through out. }\end{array}$ & VignetteText & 17 & 2 & 4 & 3.6 & 4 & 5 & 5 & 4.7 & 4.0 & 3.8 \\
\hline M Myer & MM6 & Internal & Content & \begin{tabular}{|l|} 
Recessed Non- \\
Planar Lensed \\
Lumininaires with \\
low Ballast \\
Factor and \\
Recessed \\
Compact \\
Fluorescent \\
Lensed Wall- \\
washers \\
(Office) \\
\end{tabular} & \begin{tabular}{|l|}
$\begin{array}{l}\text { Conferenc } \\
\text { e room }\end{array}$ \\
\end{tabular} & & & Approach/Concept & \begin{tabular}{|l|} 
\\
We should be consistent \\
with units. In the Lighting \\
Power Density text, we \\
write out W/ft2 and later we \\
write sf (i.e., "meeting \\
rooms smaller than 300 sf, \\
or where ceiling"
\end{tabular} & VignetteText & 17 & 2 & 4 & 3.6 & 4 & 5 & 5 & 4.7 & 4.0 & 3.8 \\
\hline M Myer & MM222 & Internal & Content & \begin{tabular}{|l|} 
Recessed 1'x4' \\
Non-planar \\
lensed \\
luminaires
\end{tabular} & & & corridors & \begin{tabular}{|c|} 
Strategy/Luminaires \\
\end{tabular} & \begin{tabular}{|l|} 
"Significantly more efficient \\
than those with open-celled \\
parabolic louvers" is \\
actually not correct. In \\
terms of luminaire \\
efficiency is roughly the \\
same as a parabolic. Also, \\
why only compare to a \\
parabolic. I recommend, \\
"they are significantly more \\
effective than other types of \\
recessed troffers in \\
simultaneously delivering \\
both good vertical and \\
horizontal illuminance"
\end{tabular} & Vignette Text & 17 & 2 & 4 & 3.6 & 4 & 5 & 5 & 4.7 & 4.0 & 3.8 \\
\hline
\end{tabular}




\begin{tabular}{|c|c|c|c|c|c|c|c|c|c|c|c|c|c|c|c|c|c|c|c|c|}
\hline $\begin{array}{l}\text { Commenter } \\
\text { ID \# }\end{array}$ & $\begin{array}{l}\text { Comment } \\
\text { Reference } \\
\#\end{array}$ & $\begin{array}{l}\text { Commenter } \\
\text { Category }\end{array}$ & $\begin{array}{l}\text { Comment } \\
\text { Type }\end{array}$ & Space Type & $\mid \begin{array}{l}\text { Ceiling } \\
\text { Height/ } \\
\text { Total Area }\end{array}$ & \begin{tabular}{|l}
$\#$ \\
workstations/ \\
partition \\
height (open \\
office only) \\
\end{tabular} & $\left|\begin{array}{l}\text { Daylight } \\
\text { Input (if } \\
\text { applicable) }\end{array}\right|$ & $\begin{array}{l}\text { Reference webpage } \\
\text { or other location } \\
\text { (see } \\
\text { "Reference.doc") }\end{array}$ & $\begin{array}{l}\text { Comment Description: } \\
\text { Issues, Suggestions \& } \\
\text { Requests }\end{array}$ & Action/Solution & \begin{tabular}{|l|} 
Status \\
or \\
Ticket \#
\end{tabular} & $\begin{array}{c}\text { Frequenc } \\
\mathrm{y}(20 \%)\end{array}$ & $\begin{array}{c}\text { Importance } \\
\text { of comment } \\
(80 \%)\end{array}$ & \begin{tabular}{|c|} 
Sum \\
$(100 \%)$
\end{tabular} & $\begin{array}{c}\text { Ease of } \\
\text { Implementatio } \\
\mathrm{n}(30 \%)\end{array}$ & $\begin{array}{c}\text { Duration } \\
(40 \%)\end{array}$ & $\begin{array}{c}\text { Risk/ } \\
\text { Complicatio } \\
\mathrm{n}(30 \%)\end{array}$ & \begin{tabular}{|} 
Sum \\
$(100 \%)$
\end{tabular} \mid & \begin{tabular}{|c|} 
Short Term \\
$(40 \%$ \\
Importance) \\
$(60 \%$ Feasibility) \\
\end{tabular} & $\begin{array}{c}\text { Long Term } \\
(85 \% \\
\text { Importance) } \\
\text { (15\% Feasibility) }\end{array}$ \\
\hline M Myer & MM601 & Internal & Content & \begin{tabular}{|l|} 
Perimter \\
Recessed CFLS
\end{tabular} & & & & $\begin{array}{l}\text { Strategy/Maintenan } \\
\text { ce }\end{array}$ & $\left|\begin{array}{l}\text { "Unit wall washers" does } \\
\text { not sound right. A linear } \\
\text { wall washer unless } \\
\text { continous is also a unit wall } \\
\text { washer. Also is that really a } \\
\text { maintennance issue? }\end{array}\right|$ & VignetteText & 17 & 2 & 4 & 3.6 & 4 & 5 & 5 & 4.7 & 4.0 & 3.8 \\
\hline M Myer & MM116 & Internal & Content & \begin{tabular}{|l|} 
Recessed Slot \\
Luminaires \\
Parallel to Desk \\
with \\
Contiunous \\
Linear Wall- \\
washers (office)
\end{tabular} & & & & Approach/Concept & $\begin{array}{l}\text { Bullet: Lumianires designed } \\
\text { for wall washing disribute } \\
\text { light... seems out of place } \\
\text { to me }\end{array}$ & VignetteText & 17 & 2 & 4 & 3.6 & 4 & 5 & 5 & 4.7 & 4.0 & 3.8 \\
\hline M Myer & MM105 & Internal & Content & \begin{tabular}{|l|} 
Recessed Slot \\
Luminaires \\
Parallel to Desk \\
with \\
Contiunous \\
Linear Wall- \\
washers (office)
\end{tabular} & & & & Luminaires C & $\begin{array}{l}\text { Continuous is a dangerous } \\
\text { word. It will really mess } \\
\text { with your LPD. }\end{array}$ & VignetteText & 17 & 2 & 4 & 3.6 & 4 & 5 & 5 & 4.7 & 4.0 & 3.8 \\
\hline M Myer & MM351 & Internal & Content & \begin{tabular}{|l|} 
Suspended \\
Direct/Indirect \\
Luminaires with \\
low BF ballasts \\
\end{tabular} & $9^{\prime}$ & $\mathrm{PO}$ & & $\begin{array}{l}\text { Strategy/Luminaires } \\
\text { B }\end{array}$ & $\begin{array}{l}\text { I do not like the defintion o } \\
\text { fhtis luminaire. I would add } \\
\text { a period after "ambient } \\
\text { lighting" }\end{array}$ & \begin{tabular}{|l|} 
VignetteText \\
\end{tabular} & 17 & 2 & 4 & 3.6 & 4 & 5 & 5 & 4.7 & 4.0 & 3.8 \\
\hline M Myer & MM310 & Internal & Content & \begin{tabular}{|l|} 
Recessed Non- \\
Planar Lensed \\
Lumianire with \\
High BF \\
Ballasts (Office)
\end{tabular} & $9^{\prime}$ & PO & & \begin{tabular}{|l|} 
Approach/Concept \\
\end{tabular} & $\begin{array}{l}\text { I would modify the text to } \\
\text { say that the use of the HBF } \\
\text { system allows for less } \\
\text { luminaires and thus the } \\
\text { lower connected load. }\end{array}$ & VignetteText & 17 & 2 & 4 & 3.6 & 4 & 5 & 5 & 4.7 & 4.0 & 3.8 \\
\hline M Myer & MM370 & Internal & Content & \begin{tabular}{|l|} 
Recessed Non- \\
Planar Lensed \\
Lumianire with \\
low BF Ballasts \\
(Office)
\end{tabular} & $9^{\prime}$ & PO & & Approach/Concept & $\begin{array}{l}\text { I would reinforce what high } \\
\text { ballast factor is in the text. }\end{array}$ & VignetteText & 17 & 2 & 4 & 3.6 & 4 & 5 & 5 & 4.7 & 4.0 & 3.8 \\
\hline M Myer & MM384 & Internal & Content & \begin{tabular}{|l|} 
Recessed Non- \\
Planar Lensed \\
Lumianire with \\
low BF Ballasts \\
(Office)
\end{tabular} & $9^{\prime}$ & PO & & $\begin{array}{l}\text { Strategy/Maintenan } \\
\text { ce }\end{array}$ & \begin{tabular}{|} 
Isn't minimizing the number \\
of ballasts contradictory \\
here? If I use a LBF here, \\
what should I use in the \\
open plan offices?
\end{tabular} & Vignette Text & 17 & 2 & 4 & 3.6 & 4 & 5 & 5 & 4.7 & 4.0 & 3.8 \\
\hline M Myer & MM618 & Internal & Content & \begin{tabular}{|l} 
Perimeter \\
Recessed \\
Linear \\
Fluorescent
\end{tabular} & & & & Approach/Concept & $\begin{array}{l}\text { It is hard to describe a } \\
\text { fixture that is less than } 60 \% \\
\text { efficient as more efficient. } \\
\text { Consider more effective? }\end{array}$ & VignetteText & 17 & 2 & 4 & 3.6 & 4 & 5 & 5 & 4.7 & 4.0 & 3.8 \\
\hline M Myer & MM125 & Internal & Content & \begin{tabular}{|l|} 
Recessed Slot \\
Luminaires \\
Parallel to Desk \\
with \\
Contiunous \\
Linear Wall- \\
washers (office)
\end{tabular} & & & & Strategy/luminaires & $\begin{array}{l}\text { Lumianrie B says } \\
\text { continous, yet the box to } \\
\text { the right says } 12^{\prime}-0 "\end{array}$ & \begin{tabular}{|l|} 
Vignette Text \\
\end{tabular} & 17 & 2 & 4 & 3.6 & 4 & 5 & 5 & 4.7 & 4.0 & 3.8 \\
\hline
\end{tabular}




\begin{tabular}{|c|c|c|c|c|c|c|c|c|c|c|c|c|c|c|c|c|c|c|c|c|}
\hline $\begin{array}{l}\text { Commenter } \\
\text { ID \# }\end{array}$ & $\begin{array}{l}\text { Comment } \\
\text { Reference } \\
\#\end{array}$ & $\begin{array}{l}\text { Commenter } \\
\text { Category }\end{array}$ & $\begin{array}{l}\text { Comment } \\
\text { Type }\end{array}$ & Space Type & \begin{tabular}{|l|} 
Ceiling \\
Height/ \\
Total Area
\end{tabular} & \begin{tabular}{|l}
$\#$ \\
workstations/ \\
partition \\
height (open \\
office only) \\
\end{tabular} & \begin{tabular}{|l|} 
Daylight \\
Input (if \\
applicable)
\end{tabular} & $\begin{array}{l}\text { Reference webpage } \\
\text { or other location } \\
\text { (see } \\
\text { "Reference.doc") } \\
\end{array}$ & $\begin{array}{l}\text { Comment Description: } \\
\text { Issues, Suggestions \& } \\
\text { Requests }\end{array}$ & Action/Solution/ & \begin{tabular}{|l|}
$\begin{array}{l}\text { Status } \\
\text { or } \\
\text { Ticket \# }\end{array}$ \\
\end{tabular} & $\begin{array}{c}\text { Frequenc } \\
\mathrm{y}(20 \%)\end{array}$ & $\begin{array}{c}\text { Importance } \\
\text { of comment } \\
(80 \%)\end{array}$ & $\begin{array}{c}\text { Sum } \\
(100 \%)\end{array}$ & $\begin{array}{c}\text { Ease of } \\
\text { Implementatio } \\
\mathrm{n}(30 \%)\end{array}$ & $\begin{array}{c}\text { Duration } \\
(40 \%)\end{array}$ & $\begin{array}{c}\text { Risk/ } \\
\text { Complicatio } \\
\mathrm{n}(30 \%)\end{array}$ & \begin{tabular}{|c|} 
Sum \\
$(100 \%)$
\end{tabular} & \begin{tabular}{|c|} 
Short Term \\
$(40 \%$ \\
Importance) \\
$(60 \%$ Feasibility) \\
\end{tabular} & $\begin{array}{c}\text { Long Term } \\
(85 \% \\
\text { Importance) } \\
\text { (15\% Feasibility) } \\
\end{array}$ \\
\hline M Myer & MM196 & $\mid \overline{\mid l \text { Internal }}$ & Content & \begin{tabular}{|l|} 
Recessed slot \\
luminaires \\
Perpendicular \\
to Desk with \\
Compact \\
Fluorescent \\
Downlights and \\
Wall-washers \\
(Office)
\end{tabular} & & & & Strategy/luminaires & \begin{tabular}{|l|} 
Luminaire E - I think that \\
the i.e. of articulated is a bit \\
much, the clarification \\
should stop after \\
"supplment the ambient \\
lighting". I would then \\
modify the text about the \\
blind old person wanting \\
more light
\end{tabular} & VignetteText & 17 & 2 & 4 & 3.6 & 4 & 5 & 5 & 4.7 & 4.0 & 3.8 \\
\hline M Myer & MM571 & Internal & Content & \begin{tabular}{|l} 
Suspended $8^{\prime}$ \\
multi-lamp \\
$(0.69$ W/sf $)$
\end{tabular} & $10 / 5000$ & $50 / 48$ & & $\begin{array}{l}\text { Approach/Luminaire } \\
\text { s B }\end{array}$ & $\begin{array}{l}\text { This text is awkward. We } \\
\text { need to know more about } \\
\text { the use of } 8^{\prime} \text { and } 4^{\prime} \\
\text { luminaires }\end{array}$ & VignetteText & 17 & 2 & 4 & 3.6 & 4 & 5 & 5 & 4.7 & 4.0 & 3.8 \\
\hline M Myer & MM626 & Internal & Content & \begin{tabular}{|l} 
Perimeter \\
Recessed \\
Linear \\
Fluorescent
\end{tabular} & & & & \begin{tabular}{|l|} 
Strategy/Maintenan \\
ce
\end{tabular} & \begin{tabular}{|l|} 
Very little of the $1 \mathrm{st}$ \\
indented maintenance \\
paragraph is maintenanced
\end{tabular} & \begin{tabular}{|l} 
VignetteText \\
\end{tabular} & 17 & 2 & 4 & 3.6 & 4 & 5 & 5 & 4.7 & 4.0 & 3.8 \\
\hline M Myer & MM366 & Internal & Content & \begin{tabular}{|l|} 
Recessed Non- \\
Planar Lensed \\
Lumianire with \\
low BF Ballasts \\
(Office)
\end{tabular} & $9^{\prime}$ & PO & & Approach/Concept & \begin{tabular}{|l|} 
"desk top" should it be one \\
word or hyphenated?
\end{tabular} & VignetteText & 17 & 2 & 4 & 3.6 & 4 & 5 & 5 & 4.7 & 4.0 & 3.8 \\
\hline M Myer & \begin{tabular}{|l|} 
MM74 \\
\end{tabular} & Internal & Content & \begin{tabular}{|l|} 
Recessed Slot \\
Lumianirs \\
parallel to desk \\
with compact \\
fluorescent wall- \\
washers (office)
\end{tabular} & & & & Approach/Concept & \begin{tabular}{|} 
"full-time occupant" should \\
be hyphenated
\end{tabular} & VignetteText & 17 & 2 & 4 & 3.6 & 4 & 5 & 5 & 4.7 & 4.0 & 3.8 \\
\hline M Myer & MM10 & Internal & Content & \begin{tabular}{|l|} 
Recessed Non- \\
Planar Lensed \\
Luminaires with \\
low Ballast \\
Factor and \\
Recessed \\
Compact \\
Fluorescent \\
Lensed Wall- \\
washers \\
(Office)
\end{tabular} & \begin{tabular}{|l|}
$\begin{array}{l}\text { Conferenc } \\
\text { e room }\end{array}$ \\
\\
\end{tabular} & & & Approach/Criteria & Hyphenate "Max-to-min" & VignetteText & 17 & 2 & 4 & 3.6 & 4 & 5 & 5 & 4.7 & 4.0 & 3.8 \\
\hline M Myer & MM563 & Internal & Content & $\begin{array}{l}\text { Suspended } 8^{\prime} \\
\text { multi-lamp } \\
(0.69 \text { W/sf })\end{array}$ & $10 / 5000$ & $50 / 48$ & & Approach/Criteria & $\begin{array}{l}\text { Hyphenate "Task-to- } \\
\text { immediate" }\end{array}$ & \begin{tabular}{|l|} 
VignetteText \\
\end{tabular} & 17 & 2 & 4 & 3.6 & 4 & 5 & 5 & 4.7 & 4.0 & 3.8 \\
\hline M Myer & MM597 & $\mid$ Internal & Content & \begin{tabular}{|l|} 
Perimter \\
Recessed CFLs
\end{tabular} & & & & Approach/Criteria & Hyphenate "Task-to-wall" & \begin{tabular}{|l|} 
VignetteText \\
\end{tabular} & 17 & 2 & 4 & 3.6 & 4 & 5 & 5 & 4.7 & 4.0 & 3.8 \\
\hline M Myer & MM574 & Internal & Content & $\begin{array}{l}\text { Suspended } 8^{\prime} \\
\text { multi-lamp } \\
(0.69 \text { W/sf })\end{array}$ & $10 / 5000$ & $50 / 48$ & & \begin{tabular}{|l|} 
Approach/Luminaire \\
$\mathrm{sC}$
\end{tabular} & $\begin{array}{l}\text { I am not a fan of the text } \\
\text { about the } 40 \text { years old and } \\
\text { corrective lenses }\end{array}$ & VignetteText & 17 & 2 & 4 & 3.6 & 4 & 5 & 5 & 4.7 & 4.0 & 3.8 \\
\hline
\end{tabular}




\begin{tabular}{|c|c|c|c|c|c|c|c|c|c|c|c|c|c|c|c|c|c|c|c|c|}
\hline $\begin{array}{l}\text { Commenter } \\
\text { ID \# }\end{array}$ & $\begin{array}{l}\text { Comment } \\
\text { Reference } \\
\#\end{array}$ & $\begin{array}{l}\text { Commenter } \\
\text { Category }\end{array}$ & \begin{tabular}{|l} 
Comment \\
Type
\end{tabular} & Space Type & \begin{tabular}{|l|} 
Ceiling \\
Height/ \\
Total Area
\end{tabular} & \begin{tabular}{|l}
$\#$ \\
workstations/ \\
partition \\
height (open \\
office only) \\
\end{tabular} & $\mid \begin{array}{l}\text { Daylight } \\
\text { Input (if } \\
\text { applicable) }\end{array}$ & \begin{tabular}{|l|} 
Reference webpage \\
or other location \\
(see \\
"Reference.doc")
\end{tabular} & $\begin{array}{l}\text { Comment Description: } \\
\text { Issues, Suggestions \& } \\
\text { Requests }\end{array}$ & Action/Solution & Status & $\begin{array}{c}\text { Frequenc } \\
\mathrm{y}(20 \%)\end{array}$ & $\begin{array}{c}\text { Importance } \\
\text { of comment } \\
(80 \%)\end{array}$ & \begin{tabular}{|c|} 
Sum \\
$(100 \%)$
\end{tabular} & $\begin{array}{c}\text { Ease of } \\
\text { Implementatio } \\
\mathrm{n}(30 \%)\end{array}$ & $\begin{array}{c}\text { Duration } \\
(40 \%)\end{array}$ & $\begin{array}{c}\text { Risk/ } \\
\text { Complicatio } \\
\mathrm{n}(30 \%)\end{array}$ & \begin{tabular}{|c|} 
Sum \\
$(100 \%)$
\end{tabular} & \begin{tabular}{|c|} 
Short Term \\
$(40 \%$ \\
Importance) \\
$(60 \%$ Feasibility) \\
\end{tabular} & $\begin{array}{c}\text { Long Term } \\
(85 \% \\
\text { Importance) } \\
\text { (15\% Feasibility) }\end{array}$ \\
\hline M Myer & MM379 & Internal & Content & $\begin{array}{l}\text { Recessed Non- } \\
\text { Planar Lensed } \\
\text { Lumianire with } \\
\text { low BF Ballasts } \\
\text { (Office) }\end{array}$ & $99^{\prime}$ & $\overline{\mathrm{PO}}$ & & |Strategy/Daylighting & I do not like "out-of-doors" & VignetteText & 17 & 2 & 4 & 3.6 & 4 & 5 & 5 & 4.7 & 4.0 & 3.8 \\
\hline M Myer & MM289 & Internal & Content & \begin{tabular}{|l|} 
Recessed 4' \\
Asymmetric \\
wall-wash \\
luminaires
\end{tabular} & & & corridors & Strategy/Luminaires & $\begin{array}{l}\text { I prefer "recessed into the } \\
\text { ceiling" rather than } \\
\text { "recessed above the } \\
\text { ceiling" }\end{array}$ & VignetteText & 17 & 2 & 4 & 3.6 & 4 & 5 & 5 & 4.7 & 4.0 & 3.8 \\
\hline M Myer & MM573 & Internal & Content & $\begin{array}{l}\text { Suspended } 8^{\prime} \\
\text { multi-lamp } \\
(0.69 \mathrm{~W} / \mathrm{sf})\end{array}$ & $10 / 5000$ & $50 / 48$ & & $\begin{array}{l}\text { Approach/Luminaire } \\
\text { s C }\end{array}$ & $\begin{array}{l}\text { Recommend ending the } \\
\text { description of articulated } \\
\text { after "visual tasks" }\end{array}$ & VignetteText & 17 & 2 & 4 & 3.6 & 4 & 5 & 5 & 4.7 & 4.0 & 3.8 \\
\hline M Myer & MM199 & Internal & Content & \begin{tabular}{|l} 
Add Corridors \\
Space/5' wide
\end{tabular} & & & corridors & Title Box & Should this be pluralized? & VignetteText & 17 & 2 & 4 & 3.6 & 4 & 5 & 5 & 4.7 & 4.0 & 3.8 \\
\hline M Myer & MM396 & Internal & Content & \begin{tabular}{|l|} 
Recessed Non- \\
Planar (LPD \\
0.56 W/sf) \\
\end{tabular} & $9.6 / 5000$ & $40 / 48$ & & Approach/Concept & $\begin{array}{l}\text { Shouldn't desktop be one } \\
\text { word or possibly } \\
\text { hyphenated? }\end{array}$ & VignetteText & 17 & 2 & 4 & 3.6 & 4 & 5 & 5 & 4.7 & 4.0 & 3.8 \\
\hline M Myer & MM153 & Internal & Content & \begin{tabular}{|l|} 
Recessed \\
Compact \\
Fluorescent \\
Downlights with \\
Dropped \\
Decorative \\
Diffuser and \\
Perimeter Wall- \\
Slot (office) \\
\end{tabular} & & & & Strategy/luminaires & $\begin{array}{l}\text { Luminaire } C \text { - the plural of } \\
\text { axis is axes }\end{array}$ & VignetteText & 17 & 2 & 4 & 3.6 & 4 & 5 & 5 & 4.7 & 4.0 & 3.8 \\
\hline M Myer & MM287 & Internal & Content & \begin{tabular}{|l} 
Recessed 4' \\
Asymmetric \\
wall-wash \\
luminaires
\end{tabular} & & & corridors & Approach/Criteria & \begin{tabular}{|l} 
Either every bullet should \\
be a whole sentence or not \\
just be consistent
\end{tabular} & VignetteText & 17 & 2 & 4 & 3.6 & 4 & 5 & 5 & 4.7 & 4.0 & 3.8 \\
\hline N Miller & NM8.1 & Internal & & & & & & & $\begin{array}{l}\text { Add comma to add clarity } \\
\text { to text: "skylights bring in } \\
\text { daylight, saving energy." }\end{array}$ & VignetteText & 17 & 2 & 4 & 3.6 & 4 & 5 & 5 & 4.7 & 4.0 & 3.8 \\
\hline N Miller & NM8.2 & Internal & & & & & & & \begin{tabular}{|l} 
Change "highlights" to \\
"highlight".
\end{tabular} & VignetteText & 17 & 2 & 4 & 3.6 & 4 & 5 & 5 & 4.7 & 4.0 & 3.8 \\
\hline
\end{tabular}




\begin{tabular}{|c|c|c|c|c|c|c|c|c|c|c|c|c|c|c|c|c|c|c|c|c|}
\hline $\begin{array}{l}\text { Commenter } \\
\text { ID\# \# }\end{array}$ & $\begin{array}{l}\text { Comment } \\
\text { Reference } \\
\#\end{array}$ & $\begin{array}{l}\text { Commenter } \\
\text { Category }\end{array}$ & $\begin{array}{l}\text { Comment } \\
\text { Type }\end{array}$ & Space Type & $\mid \begin{array}{l}\text { Ceiling } \\
\text { Height/ } \\
\text { Total Area }\end{array}$ & \begin{tabular}{|l|}
$\#$ \\
workstations/ \\
partition \\
height (open \\
office only) \\
\end{tabular} & $\left|\begin{array}{l}\text { Daylight } \\
\text { Input (if } \\
\text { applicable) }\end{array}\right|$ & \begin{tabular}{|l|} 
Reference webpage \\
or other location \\
(see \\
"Reference.doc")
\end{tabular} & $\begin{array}{l}\text { Comment Description: } \\
\text { Issues, Suggestions \& } \\
\text { Requests }\end{array}$ & Action/Solution & \begin{tabular}{|l|} 
Status \\
or \\
Ticket \#
\end{tabular} & $\begin{array}{c}\text { Frequenc } \\
\text { y (20\%) }\end{array}$ & $\begin{array}{c}\text { Importance } \\
\text { of comment } \\
(80 \%)\end{array}$ & \begin{tabular}{|c|} 
Sum \\
$(100 \%)$
\end{tabular} & $\begin{array}{c}\text { Ease of } \\
\text { Implementatio } \\
\mathrm{n}(30 \%)\end{array}$ & \begin{tabular}{|c|} 
Duration \\
$(40 \%)$
\end{tabular} & $\begin{array}{c}\text { Risk/ } \\
\text { Complicatio } \\
\mathrm{n}(30 \%)\end{array}$ & $\begin{array}{c}\text { Sum } \\
(100 \%)\end{array}$ & \begin{tabular}{|c|} 
Short Term \\
$(40 \%$ \\
Importance) \\
$(60 \%$ Feasibility) \\
\end{tabular} & $\begin{array}{c}\text { Long Term } \\
(85 \% \\
\text { Importance) } \\
\text { (15\% Feasibility) }\end{array}$ \\
\hline N Miller & NM5 & Internal & Content & $\begin{array}{l}\text { Pharm - Genl } \\
\text { Merch }\end{array}$ & & & & $\begin{array}{l}\text { Strategy-parallel } \\
\text { spacing-controls }\end{array}$ & $\begin{array}{l}\text { Change "point of sales" to } \\
\text { "point-of-sales". Same for } \\
\text { "after hours." It's clearer } \\
\text { with the hyphen. } \\
\text { What does "demand } \\
\text { reduction can be } \\
\text { accommodated" mean in } \\
\text { the Nighttime dimming } \\
\text { section? There's no } \\
\text { demand reduction needed } \\
\text { at night. Or, does this } \\
\text { mean the dimming } \\
\text { capability also allows for } \\
\text { demand reduction during } \\
\text { peak hours when the utility } \\
\text { is in trouble? }\end{array}$ & VignetteText & 17 & 2 & 4 & 3.6 & 4 & 5 & 5 & 4.7 & 4.0 & 3.8 \\
\hline M Myer & MM651 & Internal & Content & \begin{tabular}{|l|} 
Controls \\
Strategies: \\
Vacancy \\
Sensors, \\
Daylight \\
Harvesting \\
\end{tabular} & & & & Approach & $\begin{array}{l}\text { Why not more information } \\
\text { about daylighting in the } \\
\text { Approach tab? }\end{array}$ & VignetteText & 17 & 2 & 4 & 3.6 & 5 & 5 & 5 & 5 & 4.2 & 3.8 \\
\hline
\end{tabular}




\begin{tabular}{|c|c|c|c|c|c|c|c|c|c|c|c|c|c|c|c|c|c|c|c|c|c|c|}
\hline Commenter ID \# & $\begin{array}{c}\text { Comment } \\
\text { Reference \# }\end{array}$ & $\begin{array}{l}\text { Commenter } \\
\text { Category }\end{array}$ & $\begin{array}{c}\text { Operating } \\
\text { System }\end{array}$ & $\begin{array}{l}\text { Browser \& } \\
\text { Version }\end{array}$ & Comment Type & Space Type & $\begin{array}{c}\text { Ceiling } \\
\text { Height| } \\
\text { Total } \\
\text { Area }\end{array}$ & $\begin{array}{l}g \text { \# workstations/ } \\
\text { partition height } \\
\text { (open office } \\
\text { only) }\end{array}$ & $\begin{array}{l}\text { Daylight Input } \\
\text { (if applicable) }\end{array}$ & $\begin{array}{c}\text { Reference } \\
\text { webpage or other } \\
\text { location (see } \\
\text { "Reference.doc") }\end{array}$ & $\begin{array}{c}\text { Comment Description: Issues, } \\
\text { Suggestions \& Requests }\end{array}$ & Action/Solution & $\left|\begin{array}{l}\text { Status or } \\
\text { Ticket } \#\end{array}\right|$ & $\begin{array}{c}\text { Frequency } \\
(20 \%)\end{array}$ & \begin{tabular}{|} 
Importance \\
of comment \\
$(80 \%)$
\end{tabular} & $\begin{array}{l}\text { Sum } \\
(100 \%)\end{array}$ & $\begin{array}{c}\text { Ease of } \\
\text { Implementatio } \\
\mathrm{n}(30 \%)\end{array}$ & $\begin{array}{c}\text { Duration } \\
(40 \%)\end{array}$ & $\begin{array}{c}\text { Risk } \\
\text { Complicatio } \\
\mathrm{n}(30 \%)\end{array}$ & $\begin{array}{l}\text { Sum } \\
(100 \%)\end{array}$ & $\begin{array}{c}\text { Short Term } \\
(40 \% \text { Importance) } \\
(60 \% \text { Feasibility) }\end{array}$ & $\begin{array}{c}\text { Long Term } \\
(85 \% \text { Importance) } \\
\text { (15\% Feasibility) }\end{array}$ \\
\hline \begin{tabular}{|l|l|} 
A Cortese \\
\end{tabular} & AC3 & \begin{tabular}{|l|} 
Architect \\
\end{tabular} & & & \begin{tabular}{|l|}
$\begin{array}{l}\text { Appearance/Aest } \\
\text { hetics }\end{array}$ \\
\end{tabular} & & & & & & \begin{tabular}{|l|} 
Ithink that the clean look and \\
limited inputs by the user will \\
(eventually) increase its utilization \\
by professionals in the field.
\end{tabular} & Opinion & & 0 & 0 & 0 & 0 & 0 & 0 & 0 & 0.0 & 0.0 \\
\hline A Cortese & AC2 & Architect & & & Ease of Use/Flow & & & & & & $\begin{array}{l}\text { The tool is intutitive and easy to } \\
\text { use. }\end{array}$ & Opinion & & 0 & 0 & 0 & 0 & 0 & 0 & 0 & 0.0 & 0.0 \\
\hline C Magee & CM3 & Owner & $\mathrm{XP}$ & $\mathrm{IE}$ & & & & & & & $\begin{array}{l}\text { This is very sophisticated, how } \\
\text { long have you been working on } \\
\text { this tool? }\end{array}$ & Opinion & & 0 & 0 & 0 & 0 & 0 & 0 & 0 & 0.0 & 0.0 \\
\hline C Magee & CM7 & Owner & $\mathrm{XP}$ & $\mathrm{IE}$ & & & & & & & $\begin{array}{l}\text { llike the feedback that you get } \\
\text { from the various options in the } \\
\text { tool. }\end{array}$ & Opinion & & 0 & 0 & 0 & 0 & 0 & 0 & 0 & 0.0 & 0.0 \\
\hline C Magee & CM9 & Owner & $\mathrm{XP}$ & $\mathbb{E}$ & & & & & & & $\begin{array}{l}\text { Love to get feedback from the } \\
\text { facilities folks. }\end{array}$ & Opinion & & 0 & 0 & 0 & 0 & 0 & 0 & 0 & 0.0 & 0.0 \\
\hline $\begin{array}{l}\text { C Magee } \\
\end{array}$ & CM12 & Owner & $\mathrm{XP}$ & IE & & & & & & & $\begin{array}{l}\text { We work in depth with the utilities } \\
\text { on our projects, so the end goal } \\
\text { of using this in incentive } \\
\text { programs is good. }\end{array}$ & Opinion & & 0 & 0 & 0 & 0 & 0 & 0 & 0 & 0.0 & 0.0 \\
\hline \begin{tabular}{|l|l} 
D Pogue \\
\end{tabular} & DP2 & Owner & mac & & & & & & & & $\begin{array}{l}\text { Some of the clients are starting to } \\
\text { pursue LEED CI. This will help } \\
\text { with credits. }\end{array}$ & Opinion & & 0 & 0 & 0 & 0 & 0 & 0 & 0 & 0.0 & 0.0 \\
\hline D Pogue & DP1 & Owner & mac & & & & & & & & 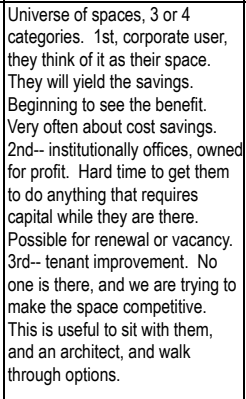 & Opinion & & 0 & 0 & 0 & 0 & 0 & 0 & 0 & 0.0 & 0.0 \\
\hline E Smith & ES2 & Owner & mac & & & & & & & & $\begin{array}{l}\text { Most projects we will look at } \\
\text { controls strategy, useful } \\
\text { background. Also true for } \\
\text { existing buildings. }\end{array}$ & Opinion & & 0 & 0 & 0 & 0 & 0 & 0 & 0 & 0.0 & 0.0 \\
\hline ESmith & ES5 & Owner & mac & & & & & & & & $\begin{array}{l}\text { The tool will help to be able to } \\
\text { show the ideas to the customer, } \\
\text { and have them feel like they are } \\
\text { part of the decision. }\end{array}$ & Opinion & & 0 & 0 & 0 & 0 & 0 & 0 & 0 & 0.0 & 0.0 \\
\hline K Abernathy & KA12 & Designer & $\mathrm{XP}$ & IE 8 & & & & & & & \begin{tabular}{|l|} 
It think it all looks terrific \\
graphically and it seems easy to \\
mannipulate. Would I use it \\
probably not but for a learning \\
tool or for someone that does not \\
do lighting every day I think it is \\
very useful.
\end{tabular} & Opinion & & 0 & 0 & 0 & 0 & 0 & 0 & 0 & 0.0 & 0.0 \\
\hline \begin{tabular}{|l|l|l|l} 
Davis & \\
\end{tabular} & LD2 & Designer & HP & Firefox & & & & & & & $\begin{array}{l}\text { This is so cool, Ijust want to } \\
\text { reinforce how great this is! }\end{array}$ & Opinion & & 0 & 0 & 0 & 0 & 0 & 0 & 0 & 0.0 & 0.0 \\
\hline M Myer & MM177 & |Internal & $\mathrm{XP}$ & Firefox 3.0 .10 & Content & \begin{tabular}{|l|} 
Recessed slot \\
luminaires \\
Perpendicular \\
to Desk with \\
Compact \\
Fluorescent \\
Downlights \\
and Wall- \\
washers \\
(Office)
\end{tabular} & & & & Luminaires/C & $\begin{array}{l}\text { llike how it starts "Recessed } \\
\text { continous T8..." }\end{array}$ & Opinion & & 0 & 0 & 0 & 0 & 0 & 0 & 0 & 0.0 & 0.0 \\
\hline M Myer & MM642 & Internal & $\mathrm{XP}$ & Firefox 3.0 .10 & Content & \begin{tabular}{|l|} 
Controls \\
Strategies: \\
Scheduling, \\
Daylight \\
Harvesting \\
\end{tabular} & & & & Controls & I love the graphics! & Opinion & & 0 & 0 & 0 & 0 & 0 & 0 & 0 & 0.0 & 0.0 \\
\hline
\end{tabular}




\begin{tabular}{|c|c|c|c|c|c|c|c|c|c|c|c|c|c|c|c|c|c|c|c|c|c|c|}
\hline Commenter ID \# & $\mid \begin{array}{c}\text { Comment } \\
\text { Reference \# }\end{array}$ & $\begin{array}{l}\text { Commenter } \\
\text { Category }\end{array}$ & $\begin{array}{c}\text { Operating } \\
\text { System }\end{array}$ & $\begin{array}{l}\text { Browser \& } \\
\text { Version }\end{array}$ & Comment Type & Space Type & $\begin{array}{l}\text { Ceiling } \\
\text { Heightl } \\
\text { Total } \\
\text { Area }\end{array}$ & $\begin{array}{c}\text { \# workstations } \\
\text { partition height } \\
\text { (open office } \\
\text { only) }\end{array}$ & \begin{tabular}{|l|} 
Daylight Input \\
(if applicable)
\end{tabular} & $\begin{array}{c}\text { Reference } \\
\text { webpage or other } \\
\text { location (see } \\
\text { "Reference.doc" }\end{array}$ & $\begin{array}{l}\text { Comment Description: Issues, } \\
\text { Suggestions \& Requests }\end{array}$ & Action/Solution & $\mid \begin{array}{l}\text { Status or } \\
\text { Ticket \# }\end{array}$ & $\begin{array}{c}\text { Frequency } \\
(20 \%)\end{array}$ & $\mid \begin{array}{c}\text { Importance } \\
\text { of comment } \\
(80 \%)\end{array}$ & $\begin{array}{l}\text { Sum } \\
(100 \%)\end{array}$ & $\begin{array}{c}\text { Ease of } \\
\text { Implementatio } \\
\mathrm{n}(30 \%)\end{array}$ & $\begin{array}{c}\text { Duration } \\
(40 \%)\end{array}$ & \begin{tabular}{|c|} 
Riskl \\
Complicatio \\
n $(30 \%)$
\end{tabular} & $\begin{array}{c}\text { Sum } \\
(100 \%)\end{array}$ & $\begin{array}{c}\text { Short Term } \\
(40 \% \text { Importance) } \\
(60 \% \text { Feasibility) }\end{array}$ & $\begin{array}{c}\text { Long Term } \\
\text { (85\% Importance) } \\
\text { (15\% Feasibility) }\end{array}$ \\
\hline N Ferzacca & NF13 & Engineer & $P C$ & & & & & & & & $\begin{array}{l}\text { Controls vignette approach looks } \\
\text { good. }\end{array}$ & Opinion & & 0 & 0 & 0 & 0 & 0 & 0 & 0 & 0.0 & 0.0 \\
\hline N Ferzacca & NF20 & Engineer & $P C$ & & & & & & & & $\begin{array}{l}\text { I can picture an architect starting } \\
\text { the process and then coming to } \\
\text { me saying hey, can we do this. }\end{array}$ & Opinion & & 0 & 0 & 0 & 0 & 0 & 0 & 0 & 0.0 & 0.0 \\
\hline N Ferzacca & NF8 & Engineer & $P C$ & & & & & & & & $\begin{array}{l}\text { Vignette details is all very } \\
\text { intuitive. }\end{array}$ & Opinion & & 0 & 0 & 0 & 0 & 0 & 0 & 0 & 0.0 & 0.0 \\
\hline N Miller & NM4.5 & Internal & Windows & Mozilla 3.5.7 & & & & & & & Good cleaning recommendations: & Opinion & & 0 & 0 & 0 & 0 & 0 & 0 & 0 & 0.0 & 0.0 \\
\hline
\end{tabular}

